\title{
On the digital road to recovery? Assessment, theory, and treatment of depression via the internet
}

Citation for published version (APA):

de Graaf, L. E. (2009). On the digital road to recovery? Assessment, theory, and treatment of depression via the internet. [Doctoral Thesis, Maastricht University]. Datawyse / Universitaire Pers Maastricht. https://doi.org/10.26481/dis.20091023lg

Document status and date:

Published: 01/01/2009

DOI:

10.26481/dis.20091023lg

Document Version:

Publisher's PDF, also known as Version of record

\section{Please check the document version of this publication:}

- A submitted manuscript is the version of the article upon submission and before peer-review. There can be important differences between the submitted version and the official published version of record.

People interested in the research are advised to contact the author for the final version of the publication, or visit the DOI to the publisher's website.

- The final author version and the galley proof are versions of the publication after peer review.

- The final published version features the final layout of the paper including the volume, issue and page numbers.

Link to publication

\footnotetext{
General rights rights.

- You may freely distribute the URL identifying the publication in the public portal. please follow below link for the End User Agreement:

www.umlib.nl/taverne-license

Take down policy

If you believe that this document breaches copyright please contact us at:

repository@maastrichtuniversity.nl

providing details and we will investigate your claim.
}

Copyright and moral rights for the publications made accessible in the public portal are retained by the authors and/or other copyright owners and it is a condition of accessing publications that users recognise and abide by the legal requirements associated with these

- Users may download and print one copy of any publication from the public portal for the purpose of private study or research.

- You may not further distribute the material or use it for any profit-making activity or commercial gain

If the publication is distributed under the terms of Article $25 \mathrm{fa}$ of the Dutch Copyright Act, indicated by the "Taverne" license above, 


\section{On the digital road to recovery?}

Assessment, theory, and treatment of depression via the Internet 
This thesis was financially supported by the RIAGG Maastricht.

On the digital road to recovery?

Assessment, theory, and treatment of depression via the Internet

L.E. de Graaf

\section{ISBN 9789052788531}

Cover and lay-out: Susanne Houwink

Production: Datawyse, Universitaire Pers Maastricht

(C) L.E. de Graaf, Maastricht 2009

All rights reserved. No part of this thesis may be reproduced or transmitted in any form or by any means, electronic or mechanical, including photocopying, recording or any information storage or retrieval system, without permission in writing from the author, or, when appropriate, from the publishers of the publications. 


\title{
On the digital road to recovery?
}

\author{
Assessment, theory, and treatment \\ of depression via the Internet
}

\begin{abstract}
PROEFSCHRIFT
ter verkrijging van de graad van doctor aan de Universiteit Maastricht, op gezag van de Rector Magnificus, prof. mr. G.P.M.F. Mols, volgens het besluit van het College van Decanen, in het openbaar te verdedigen op vrijdag 23 oktober 2009 om 14.00 uur door Lydia Esther de Graaf
\end{abstract}

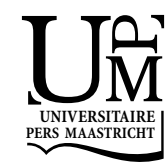




\section{Promotores}

Prof. dr. M.J.H. Huibers

Prof. dr. A. Arntz

\section{Beoordelingscommissie}

Prof. dr. M.L. Peters (voorzitter)

Prof. dr. W.J.M.J. Cuijpers (Vrije Universiteit)

Prof. dr. A.T.M. Jansen

Prof. dr. J.A. Knottnerus

Dr. F.P.M.L. Peeters 


\section{Table of contents}

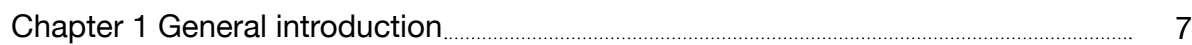

Chapter 2 Measuring dysfunctional attitudes in the general population:

the Dysfunctional Attitude Scale (form A) revised

Chapter 3 Minor and major depression in the general population:

does dysfunctional thinking play a role?

Chapter 4 Clinical and cost-effectiveness of computerised cognitive behavioural therapy for depression in primary care: design of a randomised trial

Chapter 5 Clinical effectiveness of online computerised cognitive behavioural therapy without support for depression in primary care: a randomised trial

Chapter 6 One-year follow-up results of unsupported online computerised cognitive behavioural therapy for depression in primary care: a randomised trial

Chapter 7 Economic evaluation of online computerised cognitive behavioural therapy without support for depression in primary care: a randomised trial

Chapter 8 Use and acceptability of unsupported online computerised cognitive behavioural therapy for depression and associations with clinical outcome

Chapter 9 Predicting outcome in computerised cognitive behavioural therapy for depression in primary care: a randomised trial.

Chapter 10 General Discussion 163

Summary 171

Samenvatting (Dutch summary)

References 183

Dankwoord (Acknowledgements). 197

Curriculum Vitae 201 

Chapter 1

\section{General introduction}




\section{Why use computers in the treatment of depression?}

Feeling blue from time to time is a normal part of life. Most often, such slight colourings of mood pass quickly, but in some people the sadness and hopelessness lasts for weeks, months, or even longer. Depression affects a person's thinking, behaviour, and mood. It interferes with a person's ability to participate in normal activities. Depression is highly prevalent (Bijl et al., 1998) and relapse and recurrence is very common (Judd, 1997; Keller \& Boland, 1998). It causes considerable suffering (Bijl \& Ravelli, 2000; Kruijshaar et al., 2003) and the economic burden on society is substantial (Cuijpers et al., 2007a; Luppa et al., 2007; Slobbe et al., 2006). Given the impact of depression, effective treatment is essential. The general practitioner (GP) is often viewed as a key figure in the detection and treatment of depression (Wolf \& Hopko, 2008). Although effective therapies exist, many depressed patients in primary care remain untreated (Hirschfeld et al., 1997). Pharmacotherapy, such as antidepressant medication, is only effective in more severely depressed patients (Kirsch et al., 2008) and many patients refuse medication or comply poorly (Simon et al., 1996). The chief alternative then is psychotherapy. The most widely researched form of psychotherapy is cognitive behavioural therapy (CBT), which is based on two basic components: restructuring dysfunctional thinking patterns (cognitive theory) and behavioural activation (behavioural theory).

CBT has proven to be effective in the acute phase of the disorder and in the prevention of relapse and recurrence (Butler et al., 2006; Hollon et al., 2006; Vittengl et al., 2007). Gloaguen et al. (1998) reported in their meta-analysis that CBT is more efficacious than waiting lists or placebos $(d=0.82)$ and antidepressants $(d=0.38)$ in adults with mild to moderate depression. Whether CBT is more efficacious than other types of psychotherapy is still unclear as mixed results have been found, but generally the differences seem to be small (range $d=0.03-0.24$ ) (Cuijpers et al., 2008; Gloaguen et al., 1998). Despite demonstrated effectiveness, the delivery of CBT in primary care is limited by the lack of well-trained therapists, high costs, waiting list, and patients' reluctance to enter psychotherapy. Moreover, empirical evidence for the use of CBT in primary care settings is limited, with preliminary studies yielding modest support (Wolf \& Hopko, 2008). Hence, clinicians and researchers are challenged to develop acceptable, feasible, and effective treatments for depression in primary care. Recent research has highlighted the potential for CBT to be administered in non-conventional ways, for instance via computer systems.

In our world of iPods and personal computers it makes sense to use technology to improve access to mental healthcare (Conoscenti \& Litz, 2008). Computer-assisted psychotherapy includes any computerised system designed to aid, extent, or replace traditional face-to-face therapy. Treatment tasks are delegated to a computer device. Because of its highly structured format and its clear conceptualisation, CBT lends itself very well for computerization. The level of therapist support can vary from full therapist support to minimal assistance from a nurse to no personal involvement at all. Computerised CBT (CCBT) can easily be disseminated, the costs are low compared with face-to-face psychotherapy, it can save clinicians' time, and it can shorten waiting lists. CCBT can also improve access to care for those living in remote areas and it can be used at home, and 24 hours, 7 days a week. Furthermore, computers do not suffer from limitations therapists have as human beings, such as memory problems, fatigue, or a lack of motivation. There are other advantages too: privacy and 
consistency of care and the promotion of self-activation and self-motivation (Kaltenthaler et al., 2006; Titov, 2007).

\section{A brief history of computers in mental health care}

The first general-purpose computer was developed in the mid 1940s. This computer was three meters tall and weighed approximately thirty tons. As technology has advanced rapidly over the past decades, so did the use of computers in (mental) health care. Back in the eighties of the last century, the potential applications of computers for psychiatry were already recognised (Hedluns et al., 1985). Since then, computer devices have been used to assess, diagnose, and screen for mental disorders. Other systems have been developed to consult clinicians by e-mail or fax. Online discussion groups and electronic newsletters are also valuable devices to deliver mental health care (Marks et al., 1998a). There are many applications of computer technology that concern treatment, particularly CBT. A DOS-based CCBT program developed in the late 1980s provided the impetus for many of the current CCBT studies (see Selmi et al., 1990). The first CCBT programs made use of text, multiple choice questions, case examples, and graphs to learn about changing dysfunctional cognitions (Marks et al., 1998a; Proudfoot et al., 2003). These packages remained prototypes however and have only been tested in small settings (Cavanagh \& Shapiro, 2004). Not until the last decade does it seem realistic to consider computerised interventions in mental health care. The first steps have already been made to implement CCBT into health care systems. In London, for instance, the first primary care clinic has been set up that offers several computer-aided CBT programs for anxiety and depressive disorders (Gega et al., 2004). Over the years, computerised CBT programs have been used for several mental health problems, including depression, panic, posttraumatic stress, eating disorders, phobias, chronic pain, sexual dysfunction, problem drinking, and obsessive compulsive disorders (Proudfoot et al., 2004). Packages exist for adolescents, adults, and the elderly. Another step further is the use of computerised "anti-bias" training programmes, in which patients are trained to make more fundamental and positive interpretations and associations, or to attend to positive instead of negative information (e.g. see Beard \& Amir, 2008).

\section{Which computer devices have been used in CCBT?}

A large variety of computerised systems have been used in CCBT. One of the older systems is 'Interactive Voice Response Technology' (IVR), in which the patient uses a touch-tone telephone to react to a computer system. Patients call to the computer and usually have a written manual. Interaction between patient and computer depends on the keys that patients press. Patients access various pre-recorded digitised speech fragments (Marks et al., 1998a).

Palmtops, or handheld computers, have also been incorporated into CBT. Palmtops are capable of providing flexible advice between sessions and can facilitate the generalization of therapy beyond sessions. Patients can quickly and frequently record ongoing events, symptoms, and behaviours. Built-in reminders can guide patients to apply cognitive and behavioural techniques in daily life (Anderson et al., 2004; Marks et al., 1998a; Newman et al., 1999; Williams \& Whitfield, 2001).

The latest computerised therapy programs offer high-tech, multimedia CCBT, integrating video, graphics, animations, voice-over, and interactive components, such 
as multiple-choice responding and diary completion. The combination of these various media provides a stimulating and engaging interface. Specific, active techniques of CBT are addressed as well as non-specific features known to influence clinical outcome. After each session, homework is assigned and feedback is given to reinforce learning (Cavanagh \& Shapiro, 2004). At first, these state-of-the-art CCBT programs have been made available via autonomous computers and CD-ROMs, but the latest packages can also be delivered via the Internet. It should be noted that CCBT offered via the Internet should be distinguished from Internet therapy in which therapists and patients communicate via the Internet (e.g. Interapy, Lange et al., 2005), which does not necessarily reduce the demands on therapists.

Several other computer technologies have not commonly been used in CCBT for depression, but are worth mentioning. They include text messages, interactive television, professionally assisted chat rooms, virtual reality, and video games.

\section{Caveats and pitfalls}

Despite the many advantages and possibilities of CCBT, the drawbacks should also be acknowledged. The most critical point is patient safety. Computer devices are not (yet) programmed to identify and deal with psychological crises. Patients with severe psychiatric complaints or those who are at risk of suicide should be excluded from CCBT at an early stage. Frequent monitoring by a clinician might be important to timely intervene in case of symptom deterioration or increased suicide risk (Carlbring \& Andersson, 2006; Marks et al., 1998a).

Another issue is that the use of computer devices eliminates visual cues, such as head nodding, facial expressions, and eye contact, which are important in real life communication. This problem might partly be overcome by the use of emoticons (i.e. "smilies"): typographical symbols that represent emotions (Carlbring \& Andersson, 2006).

Furthermore, computer ownership and Internet access is still not available to everyone (Carlbring \& Andersson, 2006). However, in the Netherlands almost everybody in the general adult population has access to Internet, and this will only increase in the next decade (Statistics Netherlands, www.cbs.nl).

A frequently heard caveat is that therapists might run out of work. This seems unlikely given the huge unmet demand for mental health care. Furthermore, some patients might always prefer to see a live therapist, while others might suffer from technophobia or are not capable of using computers. Moreover, CCBT is unavailable, and probably unsuitable, for certain mental health problems like schizophrenia (Marks et al., 1998a; Marks et al., 2007).

Finally, in the psychological literature it has been assumed that the positive effect that is gained from psychotherapy can mainly be attributed to common therapy factors, such as the helping relationship with a therapist (e.g. see Elkin, 1999). The therapeutic relationship is obviously missing or reduced to a minimum in computerassisted treatments. One could, therefore, argue whether CCBT can have therapeutic effects at all. However, there is no sound evidence yet as to whether or not specific therapy techniques contribute to recovery (Chambless, 2002; Luborsky et al., 2002). An attractive feature of unsupported CCBT, however, is that it can be easily dismantled into various subsets of modules to evaluate the effects of specific therapy techniques. 


\section{Does CCBT work?}

There are numerous examples of the growing evidence base for computer therapy programs for depression. The number of publications on this topic is rapidly increasing. To give an example, a quick PsycINFO search (undertaken October 2008) revealed 393 hits for 'online-therapy' entered as a major index term, of which the vast majority had been published since 2004 . Here, I will only discuss studies that involve interactive, multimedia technology, as older programs are already outdated due to the rapid development of computer technology.

Wright and colleagues (2005), for instance, investigated whether CCBT could effectively reduce the time spent with a therapist. Depressed patients were randomly allocated to traditional cognitive therapy, computerised therapy, or a waiting list. In the computerised intervention, therapist contact was reduced by half and replaced by a computer. Both active treatments were equally effective and superior to the waiting list condition during six months of follow-up. The computerised therapy thus has the potential to reduce costs and increase the accessibility of CBT. In another study, Marks et al. (2003) evaluated a clinic that provided four therapist-supported CCBT programs. Patients received access to the program that best suited their complaints. Patients significantly improved on all outcome measures and were fairly satisfied with the treatment received. Although these findings seem promising, they must be interpreted with some caution. There was no control condition and follow-up data are not available.

Recently, there has been a shift toward using the Internet in CCBT. For example, Clarke et al. (2002) investigated in a randomised trial the effects of Internet-based, unsupported CCBT for depression compared to a no-access usual care group. No significant effects were found in favour of CCBT. A possible explanation was that the website was not visited frequently enough. Some patients might find it very difficult to follow self-help regimes without some encouragement or guidance from a therapist. In a subsequent randomised trial (see Clarke et al., 2005), the authors added telephone and postcard reminders to the intervention aimed to increase the use of Internet-based CCBT. CCBT with telephone reminders and CCBT with postcard reminders were more effective than treatment as usual, although the magnitude of the effects was modest. Christensen and colleagues (2004a) also added weekly telephone contacts to two Internet-based treatments to enhance the use of the interventions. In a randomised trial, three interventions were compared: Internet-based CCBT, a psychoeducation website, and an attention placebo. Both Internet-based treatments resulted in significantly more improvement in depressive symptoms than the control condition. The psycho-education website was as effective as Internet-based CCBT in reducing depressive symptoms, but CCBT also reduced dysfunctional thinking styles, known to be an important determinant of relapse and recurrence. One-year follow-up results were promising (Mackinnon et al., 2008). Comparable results have been obtained in a study by Anderson and colleagues (2005). Improvement in depressive complaints was larger in Internet-based CCBT with minimal therapist contact than in the waiting list control group during six months of follow-up (Andersson et al., 2005). A recent metaanalysis also underscored the importance of short support in Internet-based CCBT (Spek et al., 2007a).

To our knowledge, only one study so far specifically investigated the efficacy of CCBT for depression in a primary care setting (Proudfoot et al., 2004). It was shown 
that CCBT (delivered on a personal computer located in the general practice with nurse support) was more effective than usual care by a GP in mild to moderate depression and anxiety. Moreover, CCBT in primary care was promising regarding its cost-effectiveness (McCrone et al., 2004).

\section{CCBT and cognitive behaviour theory}

Here, I will briefly discuss the theoretical underpinnings of computer-assisted CBT. CBT has integrated two originally separate theoretical approaches: the cognitive approach and the behavioural approach.

Beck is generally seen as the founding father of the cognitive theory of depression (Beck, 1972; Beck et al., 1979). According to his view, individuals vulnerable to depression have maladaptive schemas of the world, themselves and the future, which have been developed by the individual's past experiences. These maladaptive schemas remain dormant until a situation is encountered that resembles the conditions in which the original schema was learned. Once activated, these negative schema can trigger a negatively distorted pattern of information processing, which starts the downward spiral of depression (Segal et al., 1999). Dysfunctional beliefs are derived from these schemas and serve to maintain the negative schemas in spite of evidence to the contrary. The cognitive treatment that derives from this theory involves raising awareness of dysfunctional beliefs and the underlying schemas. The patient is then encouraged to replace maladaptive cognitive processes with more adaptive cognitions.

The behavioural theory of depression originates from Skinner's operant conditioning theory, which states that behaviour will be learned by obtaining rewards and avoiding punishments (Skinner, 1953). When behaviour has unpleasant or negative consequences it will be less likely to occur again. The onset of depression is associated with a decrease in pleasant events or an increase in aversive events. This may be a result of major life stressors, a decrease of available rewards and personal skills to act on the environment, or a combination of these. Social reinforcement of depressive behaviour and a lack of reinforcement or even punishment of healthy behaviour may result in a downward spiral of depression (Hopko et al., 2003; Jacobson et al., 2001). The behavioural treatment helps depressed patients to engage in pleasant activities more frequently and learns them to obtain more positive reinforcement (Cuijpers et al., 2007b).

The early cognitive and behavioural treatments did not endure as purely behavioural or cognitive. As thoughts, emotions, and behaviours are heavily intertwined, there is significant technical overlap between cognitive and behavioural techniques. Over time, they were integrated into one treatment: CBT. More recently, however, behavioural activation (BA) - a component of CBT - has been developed as a more parsimonious and complete treatment on its own (Dimidjian et al., 2006; Jacobson et al., 2001). This has put new life into the fundamental roots of behavioural models. Although the content of depressogenic thinking is not directly targeted in BA, the utility and function of dysfunctional thoughts are addressed just as in cognitive treatment (Dimidjian et al., 2006). 


\section{Aim of the current thesis}

Intuitively CCBT seems best suited for relatively mild depressive complaints because of its low intensity. Since primary care is the setting that is mainly confronted with these milder forms of depression, it is of importance to study the efficiency of CCBT in that specific context. However, most previous research has been conducted outside the reach of primary care. Other issues about CCBT have remained unresolved so far too. The question remains whether the Internet provides an efficient and acceptable venue for CCBT in primary care and whether this has the potential of being costeffective. Furthermore, studies that combine CCBT with another treatment are notably absent. Combining treatment modalities might increase the efficacy of treatment (Otto et al., 2005). These issues became the starting point of our research: a randomised trial in which we compared the effects and costs of unsupported online CCBT, usual GP care, and a combination of both. This study can contribute to the scientific and public debate regarding the undertreatment of depression. The most part of the current thesis relates to studies describing the results that were obtained during that study. Along the way, we became interested in matters related to the cognitive theory of depression on which CCBT is based. These studies will be described first.

\section{Outline of the current thesis}

The first two chapters describe two cross-sectional studies. Chapter 2 contains the results of a psychometric analysis of the Dysfunctional Attitude Scale (DAS). The DAS measures the presence and intensity of dysfunctional attitudes, a hallmark feature of depression. Chapter 3 focuses on the comparison between cognitive indices at one hand, and minor depression and major depression on the other hand to contribute to the continuum debate.

In all subsequent chapters, studies related to the randomised trial are described. Chapter 4 includes the design of the study. The recruitment strategy, the procedure, the primary and secondary outcome measures, and the planned analyses are described. Chapter 5 describes the study in which the clinical effectiveness of CCBT for depression in primary care is examined. The results during the first six months of follow-up are presented. Chapter 6 deals with the one-year follow-up results regarding the clinical effectiveness of CCBT. Long-term follow-up results of CCBT for depression have rarely been reported. It is also explored whether CCBT can prevent relapse. Chapter 7 presents the findings from the economic evaluation of CCBT, in which the cost-effectiveness is investigated from a societal perspective. Chapter 8 describes how often patients used CCBT and how acceptable they found it. Predictors of CCBT uptake are examined and it is investigated whether use and acceptability of CCBT are related to reduction in depressive severity and dysfunctional thinking. Chapter 9 describes predictors of CCBT outcome in depression at one-year of follow-up. These findings help in identifying those patients that might benefit most from CCBT.

In chapter 10, finally, the results of the different studies are integrated and discussed. Implications for clinical practice as well as methodological shortcomings are discussed and recommendations for further research are provided. 



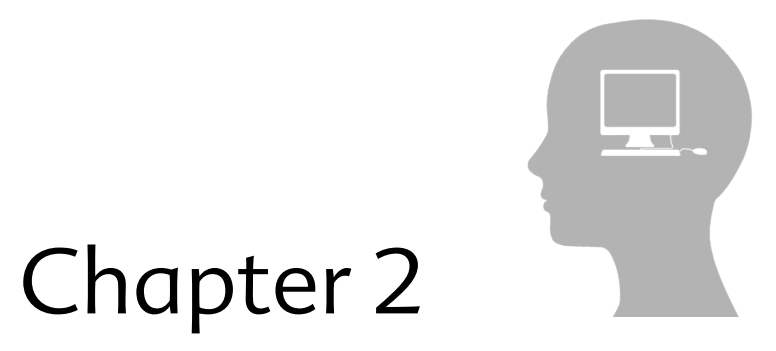

Measuring dysfunctional attitudes in the general population: the Dysfunctional Attitude Scale (form A) revised

L.E. de Graaf, J. Roelofs \& M.J.H. Huibers

This chapter is published in: Cognitive Therapy and Research (2009), 33: 345 - 355. 


\section{Abstract}

Background: The Dysfunctional Attitude Scale (DAS) was designed to measure the intensity of dysfunctional attitudes, a hallmark feature of depression. Various exploratory factor analytic studies of the DAS form A (DAS-A) yielded mixed results. The current study was set up to compare the fit of various factor models.

Methods: We used a large community sample $(N=8,960)$ to test the previously proposed factor models of the DAS-A using confirmatory factor analysis. The retained model of the DAS-A was subjected to reliability and validity analyses.

Results: All models showed good fit to the data. Finally, a two-factor solution of the DAS-A was retained, consisting of 17 items. The factors demonstrated good reliability and convergent construct validity. Significant associations were found with depression. Norm scores were presented.

Conclusions: We advocate the use of a 17-item DAS-A, which proved to be useful in measuring dysfunctional beliefs. On the basis of previous psychometric studies, our study provides solid evidence for a two-factor model of the DAS-A, consisting of 'dependency' and 'perfectionism/performance evaluation'. 


\section{Introduction}

According to Beck's view of depression (Beck, 1972; Beck et al., 1979), individuals vulnerable to depression have maladaptive schemas, which remain dormant until triggered by stressful life events. Dysfunctional beliefs reflect the content of these relatively stable schemas. In the past, many studies were unsuccessful in demonstrating this cognitive vulnerability; dysfunctional beliefs seemed to covary with depressive symptoms, suggesting state dependency rather than vulnerability (for an overview see Ingram et al., 1998). Building on Beck's cognitive model, Teasdale (1988) then suggested that dysfunctional beliefs in vulnerable individuals could only be measured in the presence of a trigger (i.e. a dysphoric mood state). During the first episode of depression, an association between dysfunctional beliefs and depressed mood is created, and dysfunctional beliefs can then be easily activated during a subsequent depressed mood (e.g. see Teasdale, 1988). Several studies have indeed found support for this 'differential activation hypothesis' using mood priming methods (Ingram et al., 1998; Lau et al., 2004; Miranda et al., 1990).

The measurement of the presence and intensity of dysfunctional beliefs in depression was advanced by the development of the Dysfunctional Attitude Scale (Weissman \& Beck, 1978). The DAS was originally designed as a measure that would reflect a general cognitive vulnerability factor to depression. However, there is some evidence to suggest that individuals vulnerable to depression may have dysfunctional beliefs only in a few, but not all, areas of their lives (e.g. Dyck, 1992; Power et al., 1995; Power et al., 1994; Sheppard \& Teasdale, 2000). Moreover, the DAS might be too general to adequately test Beck's cognitive diathesis-stress theory. Beck (1987) later proposed that specific dysfunctional beliefs will interact with particular stressors. Therefore, it is important to focus on specific rather than general dysfunctional beliefs, in research and clinical practice. If the DAS is to be used as a marker of specific vulnerabilities, subscales of the DAS measuring specific patterns of maladaptive thinking need to be identified.

Several studies have aimed to investigate the factor structure of the DAS. It is noteworthy to mention that the original form of the DAS, which consists of 100 items, has been refined into two 40-item parallel forms (i.e. DAS-A and DAS-B) by Weissman (1979). Previous research has predominantly relied on the DAS-A. Consequently, most research on the psychometric properties of the DAS has been done with the DAS-A.

The DAS-A has been subjected to exploratory factor analysis by various researchers, which yielded mixed results. Two-factor (e.g. Cane et al., 1986; Imber et al., 1990; Raes et al., 2005; Vaglum \& Falkum, 1999), three-factor (e.g. Power et al., 1994), and four-factor (e.g. Chioqueta \& Stiles, 2006; Oliver \& Baumgart, 1985; Parker et al., 1984) solutions of the DAS-A have been proposed. Moreover, some studies experienced difficulties in determining the number of factors to retain (e.g. Floyd et al., 2004). There are a number of methodological issues that might explain the variability in results from psychometric studies. First, most studies relied on the eigenvalue $>$ 1.0 or the Scree test to determine the number of factors to retain (e.g. Chioqueta \& Stiles, 2006; Floyd et al., 2004; Raes et al., 2005; Vaglum \& Falkum, 1999). These methods have been criticised for being too subjective and possibly leading to an overextraction of the number of factors (see Zwick \& Velicer, 1986). Second, the reversely keyed items in the DAS-A might be problematic. In different factor models (i.e. Chioqueta \& Stiles, 2006; Oliver \& Baumgart, 1985; Power et al., 1994) these items load 
on one factor, possibly representing a 'method' factor rather than a content factor. Third, some studies have included too few individuals to properly conduct exploratory factor analysis (e.g. Floyd et al., 2004; Oliver \& Baumgart, 1985; Parker et al., 1984; Power et al., 1994; Raes et al., 2005). It has been recommended to have at least 300 cases, and 1000 cases is regarded as excellent (Comrey \& Lee, 1992; Field, 2000). Regarding confirmatory factor analysis, many fit indices are favourably influenced by having larger sample sizes, desirably more than 200 cases (Marsh et al., 1988; Marsh et al., 1998). However, it has been difficult for researchers to determine a rule of thumb regarding the ratio of sample size to number of indicators (e.g. see Meade \& Bauer, 2007). Despite this variability, there seems to be some consistency with respect to the content of the obtained factors across studies. That is, there are two strong factors representing 'performance or achievement' and '(need for) approval by others'.

Taken together, there is a need for large-scale studies that rely on more stringent methods for examining the psychometric properties of the DAS-A. Confirmatory factor analysis is a more stringent procedure for testing the factor structure of an instrument than exploratory factor analysis, since it relies on a priori information and provides multiple goodness-of fit indices. Therefore, we will subject previously proposed factor models to confirmatory factor analysis with data from a large community sample. To the authors' best knowledge this is the first confirmatory factor analytic investigation of the DAS-A. We will subject the best fitting model of the DAS-A to reliability and validity analyses. We will establish the internal consistency and convergent construct validity. Norm-scores will be assessed and we will explore the extent to which the final model of the DAS-A is associated with depression, controlling for demographic factors. We will use demographic factors that were found to be significant correlates of depression in a large epidemiological community-based study conducted in the Netherlands (i.e. NEMESIS, Bijl et al., 1998). In line with other studies (Blazer et al., 1994; Kessler et al., 1997), they found female sex, middle age (35-44), low educational level, being occupationally disabled or without paid employment, and living without a partner to be associated with depression. 


\section{Method}

\section{Participants and procedure}

Data were collected as part of a large-scale screening program to recruit participants for a study, in which the effectiveness of computerised cognitive behavioural therapy for depression will be investigated. A random selection of individuals in the general population (age 18-65) was sent an invitation letter to complete a screening questionnaire via the Internet. Six municipalities in the Southern part of the Netherlands cooperated by providing names and addresses of their residents. The online screening was only accessible by using the unique login codes provided in each invitation letter, which could be used just once. This large Internet-based screening was completed by 8,960 (full response rate $8 \%$ ) individuals in the Dutch general population. We compared the demographic variables of our sample and the population in the Southern part of the Netherlands (Statistics Netherlands, www.cbs.nl). No major discrepancies on demographic variables could be detected.

The screening questionnaire consisted of variables concerning depression, dysfunctional attitudes and demographic data. The Medical and Ethical Committee approved the study protocol. Individuals were not compensated for participation.

\section{Measures}

Data collection was cross-sectional and took place via the Internet. All participants completed the Dysfunctional Attitude Scale form A, the Diagnostic Inventory for Depression, and completed questions concerning demographic variables (i.e. age, gender, nationality, marital status, education and employment status).

\section{Dysfunctional Attitude Scale form A}

The Dysfunctional Attitude Scale form A (DAS-A) is a self-report scale designed to measure the presence and intensity of dysfunctional attitudes. The DAS-A consists of 40 items and each item consists of a statement and a 7 -point Likert scale ( 7 = fully agree; 1 = fully disagree). Ten items are reversely coded $(2,6,12,17,24,29,30,35$, 37 and 40 ). The total score is the sum of the 40 -items with a range of 40 to 280 . The higher the score, the more dysfunctional attitudes an individual possesses (Weissman \& Beck, 1978). Internal consistency, test-retest reliability, and average item-total correlations of the DAS-A were satisfactory in different samples (e.g. Cane et al., 1986; Oliver \& Baumgart, 1985). We used the Dutch version of the DAS-A translated by Raes et al. (2005), which has good psychometric properties.

\section{Diagnostic Inventory for Depression}

The Diagnostic Inventory for Depression (DID) is a 38-item self-report scale designed to measure DSM-IV symptom inclusion criteria for a major depressive episode. The DID consists of 19 symptom severity items, 3 symptom frequency items, 8 items measuring interference in daily functioning due to depression, and 8 quality-of-life items. Specified cut-offs to determine the presence or absence of each DSM-IV criterion can be used to diagnose major depressive episode. By adding up the 19 symptom severity items, the severity of depression can be assessed, ranging from 0 (no depression) to 76 (severely depressed) (Sheeran \& Zimmerman, 2002; Zimmerman et al., 2004). Psychometric properties of the DID are good in terms of internal consistency, testretest reliability, convergent and discriminant validity, and diagnostic performance 
(Sheeran \& Zimmerman, 2002; Zimmerman et al., 2006; Zimmerman et al., 2004).

Using the specified cut-offs of the DID (Zimmerman et al., 2004), which follow the DSM-IV algorithm, we were able to determine the prevalence of major depressive episode in the current sample.

\section{Analyses}

\section{Confirmatory factor analysis}

The robustness of previously published factor models was examined by conducting confirmatory factor analysis by means of LISREL (version 8.54, Jöreskog \& Sörbom, 1999). First the one-factor model of the DAS-A was tested, followed by the following seven factor models: the two-factor models of Imber et al. (1990, details were provided by Paul A. Pilkonis), Vaglum and Falkum (1999), Cane et al. (1986), and Raes et al. (2005), the three-factor model of Power et al. (1994), and the four-factor models of Chioqueta and Stiles (2006) and Parker et al. (1984). A maximum-likelihood estimation method was adopted. A number of fit indices were used to evaluate the goodnessof-fit, including (a) the Root Mean Square Error of Approximation (RMSEA); (b) the Comparative Fit Index (CFI); (c) the Non-Normed Fit Index (NNFI); (d) the Goodness of Fit Index (GFI); and (e) the Expected Cross-Validation Index (ECVI). Kelloway (1998) indicates that RMSEA values of less than .10 represent a good fit, while values below .05 represent a very good fit to the data. Furthermore, a well-fitting model should have $\mathrm{CFI}, \mathrm{NNFI}$ and GFI values above .90 (values above .95 are indicative of a good to very good fit) (Kelloway, 1998). The ECVI is a relative measure to compare competing models; the model with the lowest value has the best fit. However, since the models show a large variability in number of items, it is risky to rely on the ECVI only. Thus, based on all fit indices, the best fitting model was retained in all following analyses.

Since many studies, especially treatment studies, rely on total scores of cognitive measures, we then subjected the one-factor model of the retained DAS-A to confirmatory factor analysis. A likelihood ratio test (LRT), then, was used to compare a more complex model (the retained factor solution) with a simpler model (onefactor solution). The simpler model is a special case of the more complex model (i.e. "nested"). More specifically, under the null-hypothesis that the special model fits as well as the more general one, the difference between their chi-square-values is itself chi-square distributed with degrees of freedom equal to the difference between their degrees of freedom.

\section{Reliability, validity, and normative data of the factors}

SPSS (version 12.0.1 for Windows) was used for all analyses, and the alpha was set to .01 to decrease the likelihood of type I error. Cronbach's alpha and corrected itemtotal correlations of the factors and of the total score of the retained DAS-A were computed. Convergent construct validity was obtained by computing Pearson correlation coefficient with the severity of depression (DID). The degree to which dysfunctional attitudes could discriminate between depressed and non-depressed individuals was examined by comparing the means of the factors of the retained DAS-A. Normative data were calculated by computing quintiles of the factors as well as for the DAS-A total score. 
Associations of the DAS factors with depression severity

To examine the unique association between dysfunctional attitudes and the severity of depression, multiple linear regression analysis was performed. The outcome variable was the severity of depression as measured with the 19 symptom severity items of the DID. First, scores of the factors of the retained DAS-A were entered in the first step, controlling for demographical variables (gender, age, partner, education and occupational status) in the second step. Second, to determine the unique additional variance of the DAS-A after the variance of demographic variables has been partialled out, these analyses were performed in reverse order (i.e. demographical variables in the first step followed by DAS-A scores in the second step). All variables were standardised prior to the analyses and standardised coefficients were interpreted. 


\section{Results}

\section{Sample characteristics}

The sample consisted of 8,960 participants. The sample distribution on socio-demographic variables is shown in table 1 . Individuals were predominantly Caucasian and in the older age groups. The number of females participating was slightly higher than the number of males. The majority had received at least eleven years of education, and was currently employed. Mean DAS-A score was $137.8(S D=23.6$; range 40 - 256). The mean DID depression severity score was 8.9 ( $S D=9.7$; range 0 - 76). According to the specified cut-offs of the DID, $719(8 \%)$ individuals currently suffered from a major depressive episode.

\begin{tabular}{|c|c|c|c|}
\hline Variable & & $N$ & (\%) \\
\hline \multirow[t]{2}{*}{ Gender } & Male & 3,965 & (44.3) \\
\hline & Female & 4,995 & (55.7) \\
\hline \multirow[t]{5}{*}{ Age } & $18-25$ years & 1,052 & (11.8) \\
\hline & $26-35$ years & 1,276 & (14.3) \\
\hline & $36-45$ years & 2,148 & $(24.0)$ \\
\hline & $46-55$ years & 2,682 & (30.0) \\
\hline & $56-65$ years & 1,795 & (20.0) \\
\hline \multirow[t]{2}{*}{ Nationality } & Dutch & 8,743 & $(97.6)$ \\
\hline & Other & 217 & (2.4) \\
\hline \multirow[t]{2}{*}{ Partner $^{a}$} & Yes & 7,901 & (89.1) \\
\hline & No & 969 & (10.9) \\
\hline \multirow[t]{3}{*}{ Education (in years) ${ }^{b}$} & $0-10$ & 2,519 & (28.6) \\
\hline & $11-14$ & 3,067 & (34.8) \\
\hline & $15+$ & 3,226 & $(36.6)$ \\
\hline \multirow[t]{5}{*}{ Occupational status $^{c}$} & Employed & 5,462 & (64.9) \\
\hline & Homemaker & 828 & (9.8) \\
\hline & Student & 673 & (8.0) \\
\hline & Occupational disability/unemployed & 1,063 & (12.6) \\
\hline & Retired & 395 & $(4.7)$ \\
\hline
\end{tabular}

\section{Confirmatory factor analysis}

First, we tested the goodness-of-fit of various factor models. Table 2 shows that the results are fairly similar for all tested models. For all models, the RMSEA values were reasonable (all values less than .10 and close to .05). The other indicators were good to very good for all models; NNFI, CFI and GFI were generally well above .90. Although sufficient, the one-factor model seemed to show the least satisfying fit. Since, the differences in fit between the other models were only marginal, we preferred retaining the most parsimonious model, i.e. a two-factor model. Another reason to retain two factors is that the three- and four-factor models might be the result of over-extraction and seemed to contain 'method' factors consisting of reversely keyed items. Inspection of the items in our sample revealed that all ten reversely keyed items showed negative and low item-total correlations (range -.40 to -.13). Although Cronbach's 
alpha was high for all 40 items (.86), the item-total correlations clearly showed conflicting results. Participants may have answered questions on autopilot. Therefore, we excluded the reversely keyed items in all next steps.

\begin{tabular}{|c|c|c|c|c|c|}
\hline Model & RMSEA (90\% Cl) & ECVI (90\% Cl) & NNFI & CFI & GFI \\
\hline $\begin{array}{l}\text { One-factor model } \\
\text { Two-factor models: }\end{array}$ & $.072(0.71-0.72)$ & $3.95(3.88-4.02)$ & .96 & .96 & .84 \\
\hline - Vaglum (1999) & $.069(.068-.071)$ & $1.14(1.10-1.18)$ & .97 & .97 & .91 \\
\hline - Imber (1990) & $.073(.072-.074)$ & $1.62(1.57-1.66)$ & .97 & .97 & .89 \\
\hline - Cane (1986) & $.066(.065-.067)$ & $1.25(1.21-1.29)$ & .97 & .97 & .91 \\
\hline - Raes (2005) & $.062(.061-.062)$ & $1.83(1.78-1.87)$ & .97 & .97 & .90 \\
\hline $\begin{array}{l}\text { Three-factor model: } \\
\text { - Power (1994) }\end{array}$ & $.065(.064-.066)$ & $1.28(1.24-1.32)$ & .97 & .97 & .91 \\
\hline Four-factor models: & & & & & \\
\hline - Parker (1984) & $.067(.066-.068)$ & $1.25(1.21-1.29)$ & .96 & .97 & .91 \\
\hline - Chioqueta (2006) & $.062(.061-.062)$ & $2.89(2.83-2.95)$ & .97 & .97 & .87 \\
\hline
\end{tabular}

Note. RMSEA = Root Mean Square Error of Approximation; ECVI = Expected CrossValidation Index; NNFI = Non-Normed Fit Index; CFI = Comparative Fit Index; GFI = Goodness of Fit Index. Bold indicates the best-fit indices.

In the next step, we closely inspected the factors and item loadings in the tested models. Two factors have emerged consistently in six of these models (i.e. Cane et al., 1986; Chioqueta \& Stiles, 2006; Imber et al., 1990; Power et al., 1994; Raes et al., 2005; Vaglum \& Falkum, 1999): 'perfectionism and performance evaluation' and 'dependency'. For the item selection, the first step was to list the items from these six studies that loaded on either one of these two factors. We only interpreted items with loadings greater than .32, which is in line with recommendations of Comrey and Lee (1992). In case of double loadings, we accepted the various author's choices regarding which item belonged to which factor. In the final step, we retained 19 items that loaded uniquely on one factor in at least five of the six studies. Although we preferred being stringent in deciding which items to retain, we did not select items that loaded on these factors in all models. Due to multiple testing, the chance increases that an item that is expected to belong to a specific factor does not load on that factor in some models.

To test the robustness of this two-factor model, we subjected the 19-item DAS-A to confirmatory factor analysis. The goodness-of-fit indicators were as follows: RMSEA $=.074(.072-.075), \mathrm{ECVI}=0.84(0.81-0.88), \mathrm{NNFI}=.97, \mathrm{CFI}=.98$, and $\mathrm{GFI}=.92$. Closer inspection of the results showed that within the first factor items 3 and 4, and items 20 and 21 had residual correlation, indicating that these items have something in common that is not shared with the remaining items of the scale. Because our purpose was to derive the most parsimonious model, we excluded item 3 and item 20 based on their lower factor loadings compared with items 4 and 21 .

In the final step, we subjected the remaining 17 items of the two-factor model of the DAS-A to confirmatory factor analysis. The results were as follows: RMSEA = $.065(.063-.066), \mathrm{ECVI}=0.52(0.49-0.54), \mathrm{NNFI}=.98, \mathrm{CFI}=.98$, and $\mathrm{GFI}=.94$. We 
also tested the goodness of fit of the DAS-A-17 as a one-dimensional model. The indicators for the one-factor model were: RMSEA $=.097(.095-.099)$, ECVI $=1.14$ $(1.10-1.18), \mathrm{NNFI}=.96, \mathrm{CFI}=.96$, and $\mathrm{GFI}=.88$. Chi-square difference between the one-factor model $\left(X^{2}(119)=7316, p<.001\right)$ and the two-factor model $\left(X^{2}(118)=3987\right.$, $p<.001$ ) was 3329 ( $d f=1, p<.05$ ), indicating that the two-factor model fit the data significantly better than the one-factor model. Moreover, the two-factor model had the lowest ECVI value, also indicating a better fit to the data. In table 3 the items and factor loadings of the retained 17-item DAS-A (DAS-A-17) are shown. We labelled the first factor 'perfectionism/performance evaluation'. This factor consisted of 11 items and contained items about perfectionism and concerns about being negatively evaluated by others on the basis of their performance. The second factor, named 'dependency', contained 6 items. The items were about a need to lean on and be supported by others, and about the dependency of approval and judgments by others in the context of interpersonal relations. ${ }^{1}$

\begin{tabular}{|c|c|c|}
\hline Item & Item description & $\begin{array}{l}\text { Factor } \\
\text { loading }\end{array}$ \\
\hline \multicolumn{3}{|c|}{ Perfectionism/performance evaluation } \\
\hline 1. & $\begin{array}{l}\text { It is difficult to be happy, unless one is good looking, intelligent, rich and } \\
\text { creative. }\end{array}$ & .52 \\
\hline 4. & If I do not do well all the time, people will not respect me. & .68 \\
\hline 8. & If a person asks for help, it is a sign of weakness. & .58 \\
\hline 9. & $\begin{array}{l}\text { If I do not do as well as other people, it means I am an inferior human } \\
\text { being. }\end{array}$ & .82 \\
\hline 10. & If I fail at my work, then I am a failure as a person. & .76 \\
\hline 11. & If you cannot do something well, there is little point in doing it at all. & .57 \\
\hline 13. & $\begin{array}{l}\text { If someone disagrees with me, it probably indicates that he does not like } \\
\text { me. }\end{array}$ & .68 \\
\hline 14. & If I fail partly, it is as bad as a complete failure. & .74 \\
\hline 15. & If other people know what you're really like, they will think less of you. & .66 \\
\hline 21. & $\begin{array}{l}\text { If I am to be a worthwhile person, I must be truly outstanding in at least } \\
\text { one major respect. }\end{array}$ & .70 \\
\hline 26. & If I ask a question, it makes me look inferior. & .68 \\
\hline \multicolumn{3}{|c|}{ Dependency } \\
\hline 19. & My value as a person depends greatly on what others think of me. & .82 \\
\hline 27. & It is awful to be disapproved of by people important to you. & .55 \\
\hline 28. & If you don't have other people to lean on, you are bound to be sad. & .45 \\
\hline 32. & If others dislike you, you cannot be happy. & .63 \\
\hline 34. & My happiness depends more on other people than it does on me. & .70 \\
\hline 38. & What other people think about me is very important. & .70 \\
\hline
\end{tabular}

${ }^{1}$ An anonymous reviewer suggested that the factor structure of the DAS-A might differ in depressed and nondepressed individuals. We therefore subjected the two-factor model of the DAS-A-17 to confirmatory factor analysis in a depressed and non-depressed subgroup separately. Depression status was based on the criteria of the DID. The results are fairly similar in both subgroups. The results for the depressed subgroup $(N=719)$ were as follows: RMSEA $=.054(.048-.060), \mathrm{ECVI}=0.61(0.53-0.69), \mathrm{NNFI}=.99, \mathrm{CFI}=.99$, and $\mathrm{GFI}=.94$. For the non-depressed subgroup $(N=8241)$ the results were: RMSEA $=.065(.063-.066)$, ECVI $=0.51(0.49$ $0.54), \mathrm{NNFI}=.97, \mathrm{CFI}=.98$, and $\mathrm{GFI}=.94$. 


\section{Reliability, validity, and normative data of the factors}

Table 4 depicts Pearson correlation coefficients (corrected for attenuation, i.e. corrected for the reliability coefficient of the instruments) between the DAS-A-17 (factors and total score) and the severity of depression (DID). With respect to the reliability, the correlation between both factors was moderate. In addition, acceptable Cronbach's alpha values were obtained for 'perfectionism/performance evaluation', 'dependency', and the DAS-A-17 total score, respectively .90, .81, and .91. The mean corrected item-total correlations were sufficient, .64 (range .51 to .77) and .58 (range .44 to .66) respectively for 'perfectionism/performance evaluation' and 'dependency'. Regarding the convergent construct validity, 'perfectionism/performance evaluation' factor and the DAS-A-17 total score were more strongly correlated with depression severity compared with 'dependency' ( $p$ s < .001; see table 4). Furthermore, table 5 shows that the depressed subgroup scored significantly higher on both factors and on the total score than the non-depressed individuals. Finally, table 6 presents normative data of the DAS-A-17 factors and total score.

Table 4 Correlation matrix of dysfunctional attitudes and depression severity $(N=8,960)$

\begin{tabular}{lllll}
\hline Scale & 1 & 2 & 3 & 4 \\
\hline 1. DID & - & .61 & .51 & .60 \\
2. DAS-A-17-P & & - & .79 & .95 \\
3. DAS-A-17-D & & & - & .87 \\
4. DAS-A-17-T & & & & -
\end{tabular}

Note. DAS-A-17 = Dysfunctional Attitude Scale with 17 items, $\mathrm{P}=$ Perfectionism/ performance evaluation, $D=$ Dependency, $T=$ total score; DID = total score of the 19 symptom severity items of the Diagnostic Inventory for Depression. Correlations are corrected for attenuation (i.e. corrected for reliability coefficient of the scales).

Table 5 Means and standard deviations of the DAS-A-17 factors and total score for the nondepressed $(N=8,241)$ and depressed $(N=719)$ subgroups

\begin{tabular}{llllll}
\multicolumn{7}{c}{ Non-depressed } & Depressed & \\
& $M$ & $S D$ & $M$ & $S D$ & $t(d f)$ \\
\hline DAS-A-17-P & 26.3 & 9.6 & 41.1 & 13.1 & $-29.6(787)^{\star}$ \\
DAS-A-17-D & 20.0 & 6.6 & 27.0 & 7.1 & $-25.5(829)^{*}$ \\
DAS-A-17-T & 46.3 & 14.7 & 68.1 & 18.5 & $-30.7(799)^{\star}$ \\
\hline Note. DAS-A-17 = Dysfunctional & Attitude & Scale with & 17 items, $\mathrm{P}=$ Perfectionism/ \\
performance evaluation (score range 7 - 77), D = Dependency (score range 7 - 42), T = total \\
score (score range 7 - 119). \\
${ }^{*} p<.001$.
\end{tabular}




\begin{tabular}{|c|c|c|c|c|}
\hline Quintile & Standardization & DAS-A-17-P & DAS-A-17-D & DAS-A-17-T \\
\hline & Low & & & \\
\hline \multirow[t]{2}{*}{1} & & 19 & 15 & 35 \\
\hline & Below average & & & \\
\hline \multirow[t]{2}{*}{2} & & 23 & 18 & 42 \\
\hline & Average & & & \\
\hline \multirow[t]{2}{*}{3} & & 27 & 22 & 49 \\
\hline & Above average & & & \\
\hline \multirow[t]{2}{*}{4} & & 35 & 27 & 60 \\
\hline & High & & & \\
\hline
\end{tabular}

\section{Associations of dysfunctional attitudes with depression severity}

Results of the multiple linear regression analysis are summarised in table 7 . The results show that the 'perfectionism/performance evaluation' and 'dependency' together account for $31 \%$ of the total variance in depression severity $(F(2)=1863.7, p<.001)$. 'Perfectionism/ performance evaluation' was significantly and substantially associated with depression. After controlling for demographic variables in the second step $(F(14)=375.9, p<.001)$, this factor remained to have the strongest association with depression. In contrast, the association between 'dependency' and depression was much smaller. It should be noticed that when demographic variables were added to the model in the second step, unemployment/occupational disability appeared more strongly associated with depression than 'dependency'.

To determine the unique additional variance of the DAS-A-17 after the variance of demographic variables has been partialled out, we repeated these analyses in reverse order. In the first step, demographic variables explained $15 \%$ of the total variance in depression $(F(12)=115.8, p<.001)$. By adding both factors of the DAS-A-17 to the model an extra $25 \%$ of the total variance could be explained $(F(14)=375.9, p<$ .001). Thus, in both regression analyses, the DAS-A-17 factors explained a substantial and significant proportion of the variance in depression.

Since it has generally been argued that 'dependency' is mainly salient in women and 'perfectionism/performance evaluation' is more often exhibited by men (Beck, 1983), gender differences might be present. Therefore, ancillary analyses were performed. First, we compared the mean scores on both factors by performing independent samples t-tests. Both factor scores were significantly higher in women than in men ( $p s<.05)$. Second, we conducted regression analyses to test interaction effects between gender and both DAS-A-17 factors as predictors of depression. The full model (i.e. demographic variables and both factors) was entered in the first step, followed by two interaction terms (i.e. gender x 'perfectionism/performance evaluation', and gender $x$ 'dependency') in the second step. The interactions were non-significant ( $p$-values well above .05). 
Table 7 Multiple linear regression analysis with depression severity as outcome variable: associations with dysfunctional attitudes and demographic variables $(N=8,960)$

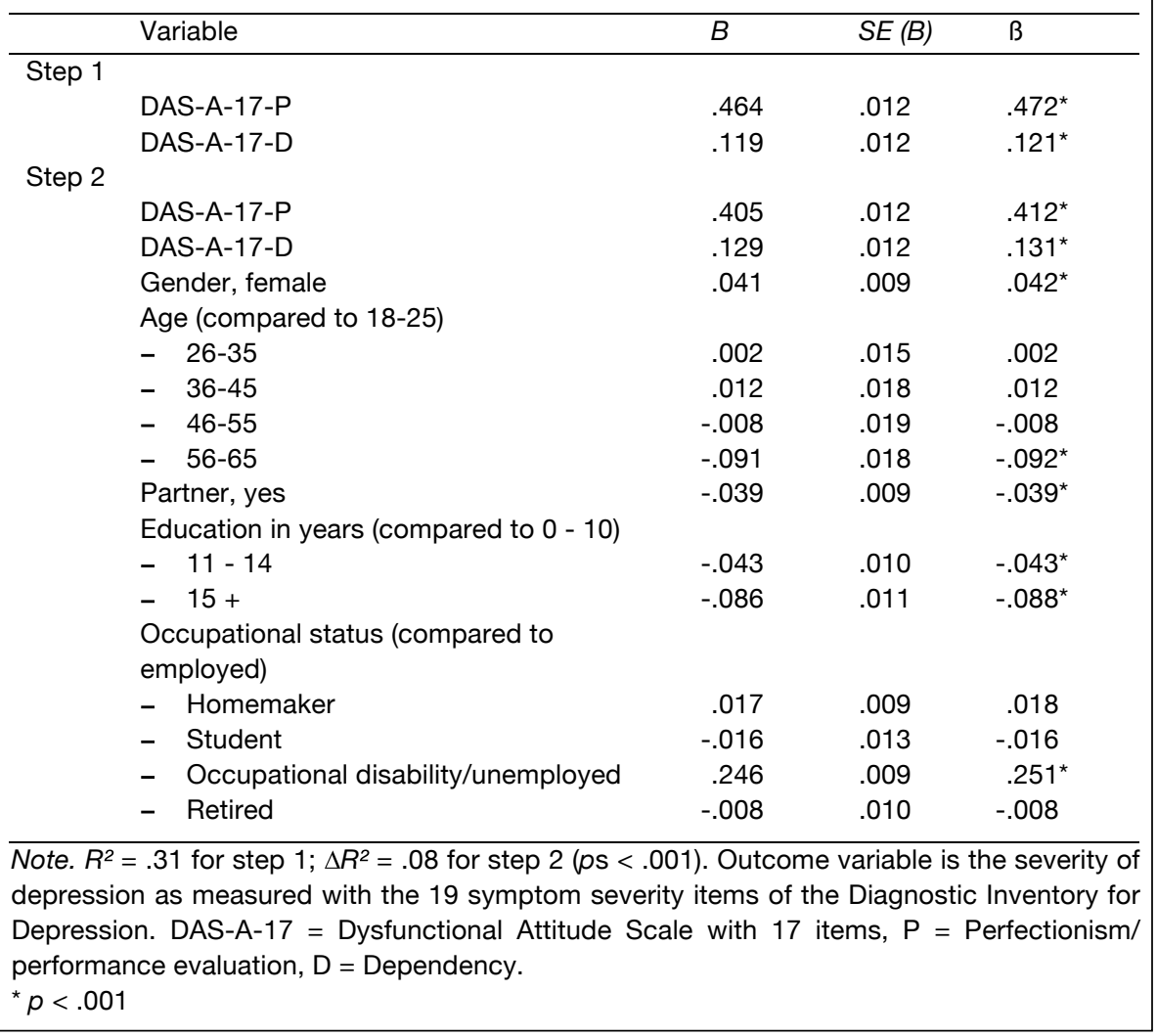




\section{Discussion}

\section{Main findings}

The present study sought to examine the psychometric properties of the DAS-A in a large community sample $(N=8,960)$. Specifically, we were able to conduct confirmatory factor analysis, which provides a methodological advance in factor analysis. We compared the fit of seven competing models of the factor structure of the DAS-A. Since all models had a good fit, we retained the most parsimonious model. Seventeen items of the original 40-item DAS-A were retained and comprised a two-factor solution: 'perfectionism/performance evaluation' and 'dependency'. Confirmatory factor analysis indicated that this two-factor solution produced good fit to the data based on several goodness-of-fit indicators. Psychometric properties of the obtained factors were sufficient, in terms of internal consistency, item-total correlations and convergent construct validity. Both factors were significantly associated with depression, controlling for demographic variables. Surprisingly, the association between 'dependency' and depression was relatively small.

\section{Previous factor-analytic studies}

One of the aims of the current study was to discern meaningful subscales of the DAS-A, which can be used as measures of specific cognitive vulnerabilities in order to more adequately test the cognitive diathesis-stress theory of Beck (1972). Therefore, we have tested several previously suggested models of the DAS-A (i.e. Cane et al., 1986; Chioqueta \& Stiles, 2006; Imber et al., 1990; Parker et al., 1984; Power et al., 1994; Raes et al., 2005; Vaglum \& Falkum, 1999). Although, all tested models had a good fit, we suggest adopting a two-factor solution for several reasons. First, two factors (i.e. 'performance or achievement' and '(need for) approval by others') have emerged across different populations in earlier studies. Second, these two factors were most interpretable and are theoretically meaningful; they have been suggested as appropriate specific dimensions of dysfunctional attitudes (Beck, 1983). Finally, factors in three- and four-factor solutions (i.e. Chioqueta \& Stiles, 2006; Oliver \& Baumgart, 1985; Parker et al., 1984; Power et al., 1994) were more difficult to interpret, and they might be the result of over-extraction due to methodological shortcomings.

While most studies focused on the psychometric properties of the DAS-A, others have examined the structure of the full 100-item DAS and the DAS-B (e.g. see Beck et al., 1991; Power et al., 1994). The authors of these two studies have both found other important factors next to 'perfectionism/performance evaluation' and 'dependency'. First, a factor labelled 'self-control' was found in the DAS-B, but did not appear in the DAS-A (Power et al., 1994). To date, 'self-control' has received relatively little attention in research on cognitive vulnerability of depression. It might be interesting for future research to elaborate more on this. Second, Beck et al. (1991) have found a general symptom factor, named 'vulnerability', reflecting a general negative view of the world. However, this factor seemed rather state dependent as compared with the 'need for approval' and 'perfectionism' factors. When specifically interested in vulnerability of depression, one might prefer to use more stable factors.

\section{Reliability}

A few comments should be made regarding the reliability of the obtained factors of the DAS-A-17. First, both factors appear to be reliable measures of specific constructs of 
dysfunctional attitudes. However, comparable to previous findings (e.g. Cane et al., 1986; Imber et al., 1990) the internal consistency is relatively smaller for 'dependency' than for 'perfectionism/performance evaluation'. The smaller number of items in the 'dependency' factor might explain this. The number of items on a scale influences Cronbach's alpha; when the number of items decreases Cronbach's alpha decreases. However, item-total correlations were also relatively smaller for 'dependency' than for 'performance evaluation'. This may suggest that dependency is a rather heterogeneous factor and may still be too broad (e.g. see Mazure et al., 2001).

Second, since total scores are often used in research and in clinical practice, the reliability of the total score of the DAS-A-17 was examined and appeared satisfactory. As the inter-correlation between both factors of the DAS-A-17 was moderate, it can even be argued that the DAS-A should preferably be used as a one-dimensional measure of dysfunctional attitudes. Moreover, the results of the confirmatory factor analysis showed that the one-factor model, of both the 40-item and 17-item DAS-A, fit the data sufficiently. Therefore, it seems justified to use the DAS-A as a one-dimensional construct. The total score might reflect a higher order construct measuring dysfunctional thinking in general. Still, the two-factor solution produced better fit to the data than the one-factor solution of the DAS-A-17.

Third, a point should be made regarding the reversely keyed items. Although usually used to prevent response tendencies, the present results suggest that reversely keyed items endorse contradictory statements. Sahin and Sahin (1992) expressed their concerns about the reversely keyed items of the DAS-A as well. In a student sample, they found that the reversely keyed items of the DAS-A formed a factor. The same problem may have emerged in previous factor analytic studies (e.g. Chioqueta \& Stiles, 2006; Oliver \& Baumgart, 1985; Power et al., 1994). Since participants had difficulties grasping the concept of these reversely keyed items, we decided to omit those items from the DAS-A. However, if the use of reversely keyed items is preferred in a questionnaire, one could elaborate the instructions for the reader and draw the participant's attention to negatively and positively stated items.

\section{Validity}

Regarding the validity, both factors showed sufficient convergent construct validity and could distinguish the depressed subgroup from the non-depressed group. Depression was significantly explained by both factors, controlling for demographic determinants of depression (see Bijl et al., 1998). The content of the obtained factors of the DAS-A-17 largely resembles two specific dimensions of cognitive vulnerability to depression: sociotropy and autonomy (Beck, 1983). Whereas sociotropy refers to a need for intimacy, affiliation and dependency, autonomy refers to as a need for goal achievement and fear of failure (Beck, 1983). Previous studies generally found stronger indications for sociotropy as risk factor to depression compared with autonomy. The present results suggest the opposite. Methodological differences may in part explain these contrasting results. First, we used a cross-sectional design, which makes it difficult to distinguish vulnerability from its manifestation in depression. 'Perfectionism/performance evaluation' may covary more closely with symptom state than 'dependency' (Beck et al., 1991; Coyne \& Whiffen, 1995). This is supported by the high correlation we found between 'perfectionism/performance evaluation' and depression severity. Second, poor scale reliability can attenuate the correlation between 
two variables. Since the alpha of the 'dependency' factor was relatively lower than for 'perfectionism/performance evaluation', this might explain its lower correlation with depression. However, we corrected for this attenuation-effect. Third, previous studies relied on other instruments to measure autonomy and sociotropy, which may differ from the DAS-A in terms of their contents (e.g. see Pincus \& Gurtman, 1995). It might be interesting to directly compare the two factors of the DAS-A-17 with the scales of the Sociotropy-Autonomy Scale. This might further support its validity. Finally, a possible explanation would have been that gender interacted with the obtained factors. It has been argued that sociotropy mainly acts as a vulnerability factor in women and autonomy in men (Beck, 1983). Some studies indeed found an interaction effect for gender and sociotropy on depression, but not for autonomy (e.g. Sato \& McCann, 1998; Shih, 2006). Although our findings suggest that there are gender differences in mean scores on both factors, the association between both factors of the DAS-A-17 and depression did not differ significantly as a function of gender. We need further research, since only few studies have examined the role of gender differences.

\section{Methodological limitations}

Several limitations of the present study should be noted. First, a potential problem in the use of online data-collection is that of providing false (demographic) information and that of multiple submissions. However, our sampling method (i.e. written invitations with unique login codes) made it possible to more reliably identify the population. Second, our full response rate was very low (8\%). Since no major discrepancies on demographic variables arose between our sample and the population in the Southern part of the Netherlands (Statistics Netherlands, www.cbs.nl), this might not be a reason for concern. However, as in most previous psychometric studies of the DAS-A, predominantly Caucasian individuals were assessed. The usefulness of the DAS-A(-17) and its factors needs to be investigated in samples representing different demographic and cultural backgrounds. Third, because of the cross-sectional nature of the study we were not able to investigate the predictive value of the obtained factors of the DAS-A. Consequently, the present study is lacking validity research. To address this issue, experimental or longitudinal designs are needed rather than cross-sectional designs. Fourth, the generalizability of the present findings might be limited due to our recruitment method. Only individuals with access to the Internet were able to participate. In line with this, one may question the equality of computerised questionnaires and paper-and-pen versions. However, there are sufficient indications that computerised and paper-and-pen questionnaires show similar construct validity (Butcher et al., 2004; Butcher et al., 2000). However, when a questionnaire is placed on the Internet this might subtly affect expected score distributions, perhaps due to increased self-disclosure or increased negative affect induced by the computer situation (Buchanan, 2003; Peterson et al., 1996). The normative data presented here might not be appropriate for the offline version of the DAS-A-17. Fifth, we relied on self-report measures and did not use a diagnostic interview. Information on past episodes of depression and other disorders, such as dysthymia, is lacking. Sixth, we used a rather heterogeneous community sample and one might question whether the factor structure of the DAS-A is similar in depressed and non-depressed individuals. However, we showed that the fit of the twofactor solution was fairly similar in a depressed and non-depressed subgroup. We think that dysfunctional attitudes can best be conceptualised as reflecting quantitative rather 
than qualitative differences among individuals (see also Gibb et al., 2004). Moreover, we were able to find a good fit despite the variability in depression status. Finally, in our proposed factor model we selected nineteen items that loaded on two common factors in at least five of six studies. Although this was carefully considered, we admit this is as a somewhat arbitrary choice. Nevertheless, we believe this is a fair approach to derive two meaningful and uniform factors.

\section{Conclusion}

In conclusion, we advocate the use of a 17-item DAS-A, which proved to be useful in defining and measuring dysfunctional beliefs in the general population. Although the 40-item DAS-A has good psychometric properties, the DAS-A-17 has several advantages over the full version. The DAS-A-17 consists of two theoretically meaningful subscales, which can be of great use in future research on cognitive vulnerability factors in depression. Furthermore, we have demonstrated that the DAS-A-17 possesses good psychometric properties in terms of model fit, reliability, and convergent construct validity. And finally, for practical reasons, many researchers and clinicians might favour a shortened version. The 17-item version can shorten administration time, while its psychometric quality is maintained. Thus, together with results from previous psychometric studies, our study provides a solid evidence-base for a specific two-factor structure of the DAS-A across settings and populations, consisting of 'dependency' and 'perfectionism/performance evaluation'. 


\section{Acknowledgements}

We thank Annie Hendriks, Greet Kellens, and Sylvia Gerhards, who assisted with data collection. Rosanne Janssen developed the infrastructure for online data-collection. Municipalities Eijsden, Meerssen, Sittard-Geleen, Valkenburg, and Maastricht sponsored the study. 


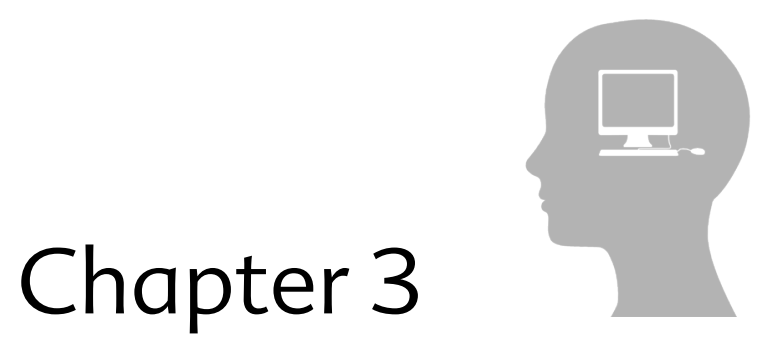

Minor and major depression in the general population: does dysfunctional thinking play a role?

L.E. de Graaf, M.J.H. Huibers, P. Cuijpers \& A. Arntz 


\section{Abstract}

It remains unclear whether minor depression is a discrete category or only differs from major depression in severity. To contribute to this continuum debate, demographic, clinical, and cognitive factors (i.e. content and form of dysfunctional thinking) were compared between minor depression (i.e. 2 - 4 symptoms), major depression with 5 to 6 symptoms, and major depression with 7 to 9 symptoms. A large community sample $(N=1129)$ was used. Differences between the three subgroups were examined as well as linear relations between number of symptoms and factors marking the severity. The findings mainly suggest that minor depression should be considered as part of continuum together with major depression. Moreover, not only the content, but also the form (i.e. extreme responding), of dysfunctional thinking seems to play a major role in depression. This finding, if replicated, may have important implications for the cognitive theory of depression. 


\section{Introduction}

Minor depression is common in the general population, with greater prevalence rates than major depression, and seriously affects quality of life, functional impairment, and health care use (Beck \& Koenig, 1996; Chen et al., 2000; Kessler et al., 1997). Minor depression is associated with considerable costs (Cuijpers et al., 2007a) and with an increased risk of developing major depression (Fogel et al., 2006). Minor depression can be defined as identical to major depression in duration, but with fewer symptoms (i.e. 2 - 4 symptoms; see research criteria of the DSM-IV). In the current study, it will be explored whether minor depression shares important correlates with major depression, with a special emphasis on cognitive factors, that is, features of dysfunctional thinking based on Beck's cognitive model of depression (Beck, 1987).

\section{The continuum issue}

Ongoing debate has focused on whether minor depression is a discrete diagnostic category or part of a continuum that differs from major depression only in severity. Resolution of this issue may contribute to the conceptualisation of the causes, correlates, and consequences of depression as well as for decisions regarding research design, statistical analysis, assessment, and treatment (Prisciandaro \& Roberts, 2005; Ruscio \& Ruscio, 2004a; Solomon et al., 2006). Moreover, resolving this controversy may have important implications for theories that posit continuity, such as Beck's cognitive theory of depression (Solomon et al., 2001). Several techniques may be used to address the continuum issue.

One approach is to compare characteristics of minor depression with those of major depression. Subtypes on the continuum should be similar in demographic characteristics, whereas factors that mark the severity (e.g. disability) should be different (see Solomon et al., 2001). Two large community-based studies (Cuijpers et al., 2004; Kessler et al., 1997) evaluated demographic and clinical correlates of minor depression relative to major depression with 5 to 6 symptoms (MDD 5-6) and major depression with 7 to 9 symptoms (MDD 7-9). Gender, age, employment (Cuijpers et al., 2004; Kessler et al., 1997), education (Cuijpers et al., 2004), and ethnicity (Kessler et al., 1997) were comparable in the three depression categories. Similar results were found in a sample of primary care patients (Backenstrass et al., 2006). Not all findings were in agreement with the continuum hypothesis. The risk for more severe depression categories was larger for females (Cuijpers et al., 2004; Kessler et al., 1997) and the risk for MiDD and MDD 5-6, but not for MDD 7-9, was lower for those with few educational years compared to those with many years of education (Kessler et al., 1997). In accordance with the continuum hypothesis, clinical characteristics of depression, such as number of previous episodes, impairment, and health care use, act in synchrony with the severity of the disorder (Backenstrass et al., 2006; Cuijpers et al., 2004; Kessler et al., 1997). However, Kessler et al. (1997) did not find this pattern for work loss days and work cutback days. Taken together, findings comparing correlates of minor and major depression seem mainly, but not completely, in support of the continuum hypothesis.

Comparing subgroups on the depression continuum has the advantage that it resembles the reality of daily practice and is thus insightful for clinical practice. However, some have argued that this approach cannot resolve the continuum controversy, because a manifest symptom can conceal a latent clinical taxon (i.e. a category) (see 
Solomon et al., 2001; Solomon et al., 2006). A more sophisticated method is the taxometric technique (Meehl, 1995). Taxometric studies on depression have yielded mixed results, some arguing in favour of the continuum hypothesis (e.g. see Prisciandaro \& Roberts, 2005), others supporting the existence of a distinction between clinical and subthreshold depression (e.g. Ruscio et al., 2007; Solomon et al., 2006). Therefore, the possibility that minor depression and major depression are discrete categorical subtypes cannot yet be ruled out.

It should be noted that the taxometric technique is not suitable for the current research. The indicators used in taxometric analysis need to assess all relevant facets of the targeted construct and they must not converge on other disorders, such as anxiety (Ruscio \& Ruscio, 2004b). It is known that dysfunctional thinking, which is the focus of the current study, may not be specific for depression (Hill et al., 1989; Hollon et al., 1986).

\section{Dysfunctional thinking: content and form}

According to Beck's cognitive model (see Beck, 1967; 1983) dysfunctional attitudes are likely to promote depressive symptomatology, when triggered by stressful life events. The presence and intensity of dysfunctional attitudes in depression has frequently been measured by the Dysfunctional Attitude Scale (DAS) (Weissman \& Beck, 1978). Several studies have shown that the content of dysfunctional thinking (i.e. numerical scores on the DAS) is associated with vulnerability to major depression (e.g. see Kwon \& Oei, 1992; Olinger et al., 1987). Others, more recently, have proposed that the form (i.e. the frequency of extreme responses on the DAS), rather than the content, of dysfunctional thinking is a cognitive vulnerability factor for relapse and poor treatment outcome in severe, chronic and recurrent depression (Beevers et al., 2003; Petersen et al., 2007; Teasdale et al., 2001). This is in accordance with the importance of dichotomous thinking within Beck's cognitive model of depression (Beck et al., 1979). This form of absolutistic thinking refers to the tendency to evaluate experiences in terms of exclusive categories.

Only few studies have reported results of dysfunctional thinking in minor depression. Those studies only focused on the content of dysfunctional thinking. It was shown that individuals who exhibit dysfunctional cognitions were more likely to experience prospective onsets of both major and minor depression than those who do not show these cognitions, but the prevalence and risk is higher for major depression than for minor depression (Alloy et al., 2000; Alloy et al., 2006). A taxometric analysis also showed that dysfunctional cognitions can best be conceptualised as reflecting quantitative rather than qualitative differences among individuals (Gibb et al., 2004). These findings are in line with the cognitive model of depression, which takes a strong dimensional perspective (Clark et al., 1999).

\section{Current study}

Taken together, literature on minor depression is growing and generally showing that minor depression shares important correlates with major depression. However, there is relatively little evidence that minor depression and major depression share features related to dysfunctional thinking. This issue will be addressed in the current study using a large community sample. It should be noted that the focus of the study was the relation of minor depression and major depression, and, therefore, a subgroup 
of participants without depressive symptoms was not included in the study. Demographic (i.e. gender, age, education, marital status and employment status), clinical (i.e. severity, current treatment for depression, quality of life and interference in daily functioning) and cognitive factors (i.e. content and form of dysfunctional thinking) will be compared between minor depression, major depression with 5 to 6 symptoms, and major depression with 7 to 9 symptoms. This classification is in line with previous studies (see also Cuijpers et al., 2004; Kessler et al., 1997) and makes it possible to create subtypes that might be a reflection of a depressive continuum (Kessler et al., 1997). In accordance with the continuum hypothesis, the leading hypothesis is that no substantial differences in demographic characteristics exist between the three depression categories. In contrast, it was expected that the clinical and cognitive factors covary linearly with severity of depression. 


\section{Method}

\section{Participants and procedure}

Data were collected as part of a large-scale screening program to recruit participants for an RCT on the effectiveness of computerised cognitive behavioural therapy for depression. Data collection was cross-sectional. A random selection of individuals in the general population (age 18-65) was sent an invitation letter to complete a screening questionnaire via the Internet. Six municipalities in the Southern part of the Netherlands cooperated by providing names and addresses of their residents. This large Internet-based screening was completed by 8,960 (full response rate $8 \%$ ) individuals in the Dutch general population. A comparison of the demographic variables of the current sample and the population in the Southern part of the Netherlands (Statistics Netherlands, www.cbs.nl) did not reveal any major discrepancies.

For the purpose of the present study, only participants with depressive symptomatology (two or more DSM-IV symptoms for depression, including one of the key symptoms) were included in the analyses $(N=1129)$. The Medical and Ethical Committee approved the study protocol. Individuals were not compensated for participation.

\section{Measures}

\section{Diagnostic Inventory for Depression}

The Diagnostic Inventory for Depression (DID) is a 38-item self-report scale designed to measure DSM-IV symptom inclusion criteria for a major depressive episode (Zimmerman et al., 2004). The DID consists of 19 symptom severity items, 3 symptom frequency items, 7 items measuring interference in daily functioning due to depression, and 9 quality-of-life items. Each item is to be rated on a 5-point Likert scale with increasing severity (range 0 - 4). Specified cut-offs to determine the presence or absence of each DSM-IV criterion can be used to diagnose major depressive episode. By adding up 6 interference-in-daily-functioning items, the severity of the interference can be assessed, ranging from 0 (no interference) to 24 (very severe interference). Similarly, quality of life can be assessed by adding up the 9 quality-of-life items $(0=$ good quality of life; 36 = poor quality of life). Psychometric properties of the DID are good in terms of internal consistency, test-retest reliability, convergent and discriminant validity, and diagnostic performance (Sheeran \& Zimmerman, 2002; Zimmerman et al., 2006; Zimmerman et al., 2004).

\section{Beck Depression Inventory for Primary Care}

The Beck Depression Inventory for Primary Care (BDI-PC) is designed to screen for major depression (Beck et al., 1997). The BDI-PC consists of 7 items derived from the Beck Depression Inventory II, reflecting DSM-IV criteria for major depressive episode. Each item is rated on a 4 point Likert scale (0 to 3). The total score is the sum of the 7 items. The higher the score, the more severe the depression. Psychometric studies have shown high internal consistency, sufficient convergent validity, and good diagnostic differentiation (Beck et al., 1997; Steer et al., 1999; Winter et al., 1999).

\section{Dysfunctional Attitude Scale form A}

The Dysfunctional Attitude Scale form A (DAS-A) is a self-report scale designed to measure the presence and intensity of dysfunctional attitudes (Weissman \& Beck, 
1978). Each item consists of a statement and a 7-point Likert scale ( 7 = fully agree; 1 = fully disagree). The Dutch version of the DAS-A has good psychometric properties (Raes et al., 2005). An abridged version was used. Previously, various exploratory factor analytic studies yielded mixed results. In a recent study (de Graaf et al., in press-b), the fit of seven competing models was compared in confirmatory factor analysis. The most parsimonious model was retained, consisting of 17 items (viz. DAS-A-17) comprising two subscales: 'dependency' (6 items) and 'perfectionism/performance evaluation' (11 items). The score is the sum of the items. The higher the score, the more dysfunctional attitudes an individual reports. Psychometric properties of both subscales were sufficient, in terms of internal consistency (total scale: $a=.91$; dependency: $a=.81$; perfectionism: $a=.90$ ), item-total correlations (average item-total correlations for dependency: .58; perfectionism: .64) and convergent construct validity (de Graaf et al., in press-b).

Extreme response style was investigated by calculating the number of extreme responses on the DAS-A-17, as previously described by Teasdale et al. (2001). Both extreme positive responding (number of 'fully disagree' answers with dysfunctional items) and extreme negative responding (number of 'fully agree' answers with dysfunctional items) were investigated.

\section{Questionnaire without Questions}

Since extreme responding to depression related questionnaires, such as the DASA-17, might be confounded by depressive severity (i.e. the items might trigger latent dysfunctional attitudes more easily in more severe depression; see Teasdale et al., 2001), extreme response tendencies were also explored in a neutral way. A paradigm to study response tendencies was developed by Berg and Rapaport (1954): the Questionnaire without Questions (QwQ). Individuals have to choose between various answers, solely based on the presentation of the answers. Healthy individuals seem to avoid extreme responses and tend to have a preference for positive answers (Berg \& Rapaport, 1954; de Jonge \& Slaets, 2005; van IJzendoorn, 1984). Substantial testretest correlations were found (de Jonge \& Slaets, 2005). The following answering categories were used in the current study: (1) yes-uncertain-no, (2) very satisfiedsatisfied-unsatisfied-very unsatisfied, and (3) and agree-neutral-disagree. Extreme positive responding was calculated by adding up the number of extreme positive answers (i.e. yes, very satisfied, and agree). Extreme negative responding was calculated by adding up the number of extreme negative answers (i.e. no, very unsatisfied, and disagree).

\section{Other measures}

Other measures included self-reported gender, age, education, employment, marital status, treatment (antidepressants and/or psychological treatment), and duration of complaints.

\section{Classification of depression status}

The classification of depression status used in previous studies was adopted (see Cuijpers et al., 2004; Kessler et al., 1997). Individuals were classified into three mutually exclusive categories based on the presence of one of the DSM-IV key symptoms (depressed mood and/or anhedonia) and on their additional number of DSM-IV de- 
pressive symptoms as measured with the DID. The following depression status categories can be distinguished:

1. Minor depression: 2 to 4 symptoms including one key symptom (MiDD);

2. Major depression 5-6: 5 to 6 symptoms including one key symptom (MDD 5-6);

3. Major depression 7-9: 7 to 9 symptoms including one key symptom (MDD 7-9). In some analyses, MDD 5-6 and MDD 7-9 were combined into one group. This more closely resembles the reality of daily practice and might be more insightful for clinical practice. This group is then referred to as major depression (MDD).

\section{Statistical analyses}

Preliminary tests for distribution and outliers were performed. Skewness and Kurtosis indicated deviations from normality for scores regarding extreme responding on the QwQ and DAS-A-17. Only those participants who completed all measures were included in the analyses. As a result, there were no missing data. All analyses were carried out using SPSS (version 15.0.1 for Windows). The alpha was set to .01 to decrease the likelihood of type I error.

First, frequency differences between the three depression status categories in categorical variables were tested using Chi-square tests. To explore mean differences in continuous variables ANOVA's were used. In case of statistically significant effects, post hoc pairwise Tukey tests were conducted comparing the scores between each pair of groups. For the scores regarding extreme responding we used non-parametric Kruskal-Wallis tests. In case of significant effects, Mann-Whitney tests were conducted for each pair of groups. Next, effect sizes were calculated, defined as: $\left(M_{\text {group a }}-\right.$ $\left.M_{\text {group b }}\right) / \sigma_{\text {pooled }}$, where $\sigma_{\text {pooled }}=\sqrt{ }\left[\left(\sigma_{\text {group a }}^{2}+\sigma_{\text {group b }}^{2}\right) / 2\right]$ (Cohen, 1988). Small, medium, and large effect sizes are respectively .20, .50, and .80 (Cohen, 1992).

Second, the bivariate correlation between number of symptoms and continuous measures of clinical and cognitive factors were calculated. This can be seen as an indirect method of testing continuity (Flett et al., 1997). Pearson correlations were used for normally distributed variables and Spearman for non-normally distributed variables. Correlations are corrected for attenuation (i.e. corrected for reliability coefficient of the scales).

Third, to investigate the association between demographic, clinical, and cognitive factors and depression status pairwise logistic regression analyses were performed. MDD (code $=1)$ was compared with MiDD (code $=0)$, and MDD 7-9 (code =1) was compared with MDD 5-6 (code $=0)$. Variables showing significant univariate associations $(p<.05)$ with the outcome variable were entered into the model. Variables were eliminated in a backwards procedure until the model consisted of significant associations only $(p<.01)$. Gender, age, and education were always kept in the model irrespective of their statistical significance. $95 \%$ confidence intervals were calculated. All variables were dichotomised prior to the analyses in order to be more conservative and to be able to interpret the OR's. Continuous variables were dichotomised based on the mean value. Categorical variables were dichotomised as follows: education (low/medium to high) and employment status (employed/unemployed or on sick leave). 


\section{Results}

\section{Demographic and clinical characteristics}

In table 1 demographic and clinical characteristics of the three depression status categories are shown.

As to the demographic variables, the results revealed only a significant difference for employment status $(p<.001)$; with increasing number of symptoms, the number of currently employed individuals decreased. At a $p<.05$ level, a borderline significant difference was found for gender $(p=.06)$. Post hoc pairwise comparisons revealed that there were fewer men in the MDD7-9 group than in the MiDD group $\left(X^{2}(1)=5.36, p=.02\right)$.

With respect to the clinical characteristics, all variables showed statistically significant differences between the three depression status categories. Post-hoc Tukey tests showed that all pairwise comparisons were significant (all $p<.001)$. When the number of symptoms increased, the following variables increased substantially: (a) depressive severity (BDI-PC), (b) interference in daily functioning (DID), (c) use of antidepressants and psychological treatment, and (d) the number of individuals whose current episode is lasting longer than 6 months. Quality of life (DID) decreased significantly across the three depression status categories. Effect sizes for depressive severity, interference in daily functioning, and quality of life were medium between MiDD and MDD 5-6, while they were large between MDD 5-6 and MDD 7-9 (see table 1).

\section{Dysfunctional thinking and depression status}

The three depression status categories differed significantly in dysfunctional thought content and extreme responding on all measures (see table 2). Tukey post-hoc comparisons revealed that both 'dependency' and 'perfectionism' became significantly more severe with increasing number of symptoms ( $p s<.01)$, but that the difference between MiDD and MDD 5-6 was just significant for 'dependency' $(p=.01)$.

For scores regarding extreme responding, pairwise Mann-Whitney tests were conducted. Significantly higher scores for extreme negative responding on both the DAS-A-17 and the QwQ were obtained for MDD 7-9 relative to MDD5-6 and MiDD (ps < .01). The difference in extreme negative responding between MiDD and MDD5-6 was significant on the DAS-A-17 $(p<.01)$, but not on the QwQ $(p=.06)$. In contrast, scores for extreme positive responding on the DAS-A-17 and QwQ increased when the number of symptoms increased over the three depression status groups $(p<.01$ for all pairwise comparisons).

All effect sizes for dysfunctional thought content and extreme responding were small between MiDD and MDD 5-6 and between MDD 5-6 and MDD 7-9 (see table 2). The largest effect size was obtained for extreme negative responding on the $Q w Q$ between MDD 5-6 and MDD 7-9. 


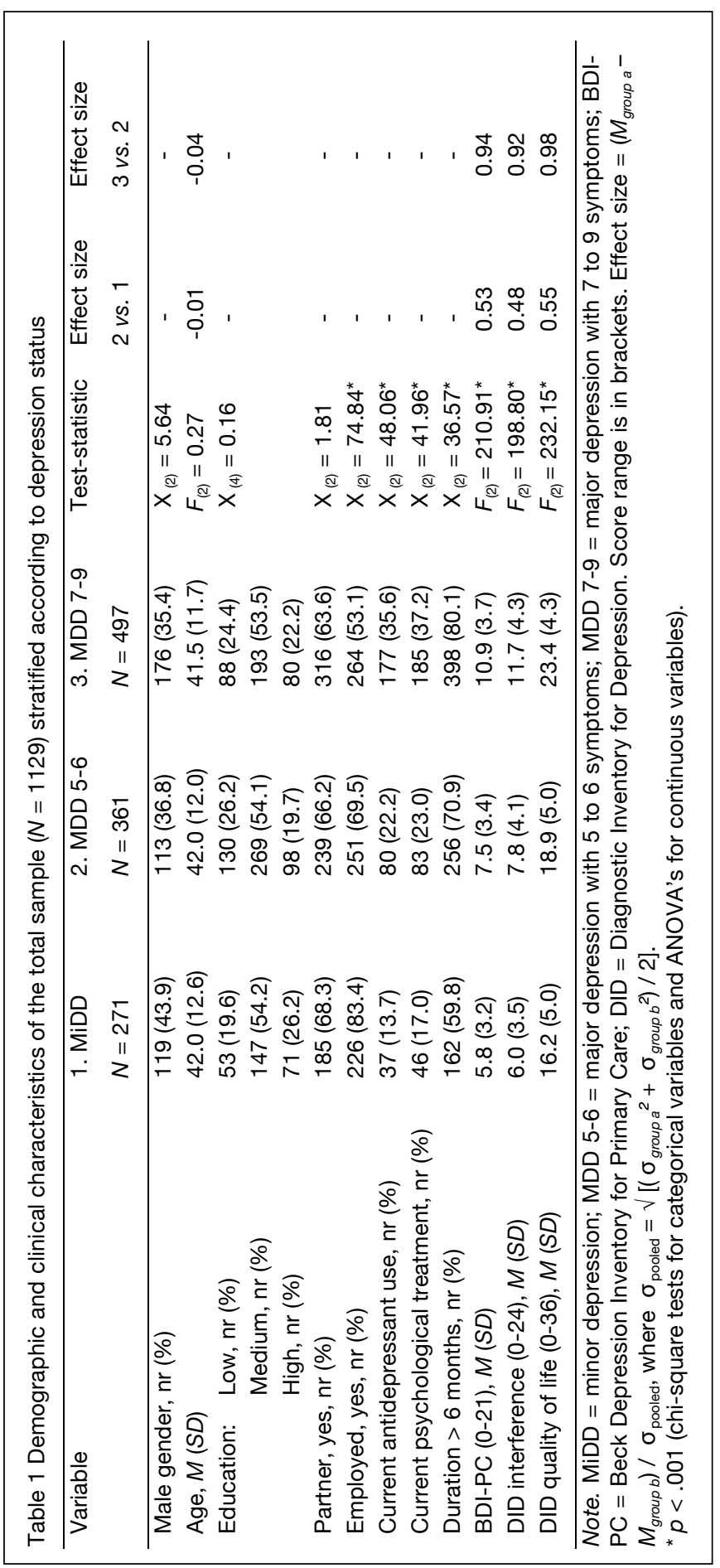




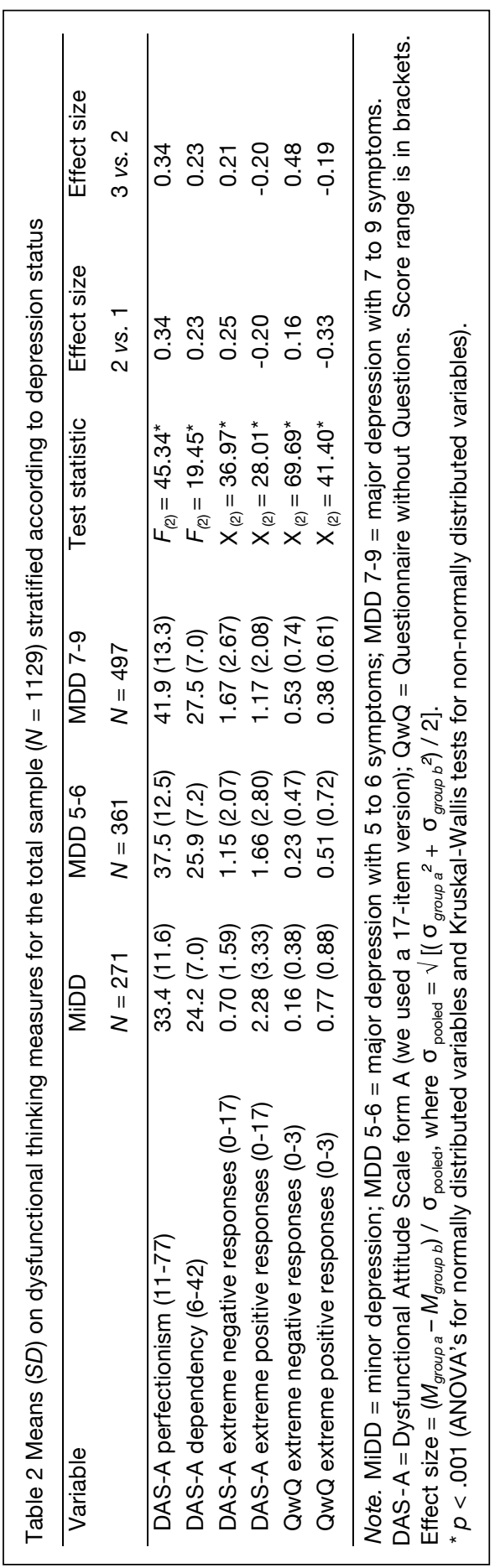




\section{Associations between demographic, clinical, and cognitive factors and depression status}

As can be seen in table 3, large correlations were found between number of symptoms (range 2-9) and the clinical factors (i.e. depressive severity, interference in daily functioning, and quality of life). Correlations with dysfunctional thought content and extreme responding were relatively smaller. Negative correlations were found for extreme positive responding on the DAS-A-17 and the QwQ.

\begin{tabular}{|lc|}
\hline \multicolumn{2}{l}{$\begin{array}{l}\text { Table } 3 \text { Correlations of number of depressive symptoms with clinical and cognitive factors in the } \\
\text { total sample }(N=1129)\end{array}$} \\
\hline Scale & Correlation coefficient \\
\hline BDI-PC & $.72^{\star}$ \\
DID interference & $.68^{*}$ \\
DID quality of life & $.72^{\star}$ \\
DAS-A perfectionism & $.35^{\star}$ \\
DAS-A dependency & $.26^{*}$ \\
DAS-A extreme negative responses & $.25^{\star}$ \\
DAS-A extreme positive responses & $-.18^{\star}$ \\
QwQ extreme negative responses & $.43^{*}$ \\
QwQ extreme positive responses & $-.31^{*}$ \\
\hline Note. BDI-PC = Beck Depression Inventory for Primary Care; DID = Diagnostic Inventory for \\
Depression; DAS-A = Dysfunctional Attitude Scale form A (we used a 17-item version); QwQ = \\
Questionnaire without Questions. Pearson correlations for normally distributed variables and \\
Spearman for non-normally distributed variables. Correlations are corrected for attenuation. \\
* $p<.01$
\end{tabular}

Table 4 presents the results of multiple logistic regression analyses with the depression status categories as dependent variables. After backward elimination, only significant associations are reported, together with gender, age, and education. In the first model, MDD (code $=1$ ) was compared with MiDD (code $=0)$. Six variables were significantly associated with $\operatorname{MDD}\left(X^{2}(8)=240.0, p<.001\right)$. Men had significantly lower odds for MDD compared with women. The odds for MDD were significantly lower for participants who were currently employed compared with those without employment. Other variables that significantly distinguished MDD from MiDD were: high interference in daily functioning (DID), poor quality of life (DID), high perfectionism (DAS-A$17)$, and high extreme positive responding (QwQ).

In the second logistic model, MDD 7-9 was (code =1) compared with MDD 5-6 $($ code $=0)$. Four factors could significantly distinguish MDD 7-9 from MDD 5-6 ( $X^{2}(7)$ $=164.8, p<.001$ ): being employed, high interference in daily functioning (DID), poor quality of life (DID), and high extreme negative responding (QwQ). 
Table 4 Results from multiple logistic regression analyses: associations between demographic, clinical, and cognitive factors and depression status

\begin{tabular}{|c|c|c|c|}
\hline Variables & $B(S E)$ & Wald $(d f=1)$ & OR $(95 \% \mathrm{Cl})$ \\
\hline \multicolumn{4}{|l|}{$\operatorname{MDD}(N=858)$ vs. $\operatorname{MiDD}(N=271)^{\mathrm{a}}$} \\
\hline Gender (male) & $-0.56(0.16)$ & $11.80^{*}$ & $0.57(0.42-0.79)$ \\
\hline Age (>42 years) & $0.01(0.16)$ & 0.01 & $1.00(0.73-1.37)$ \\
\hline Education (medium to high) & $-0.23(0.19)$ & 1.41 & $0.80(0.55-1.16)$ \\
\hline Employment (employed) & $-0.91(0.20)$ & $21.14^{\star \star}$ & $0.41(0.28-0.60)$ \\
\hline DID interference (score>10) & $0.93(0.20)$ & $21.87^{\star \star}$ & $2.54(1.72-3.76)$ \\
\hline DID quality of life (score>21) & $1.25(0.19)$ & $44.18^{\star \star}$ & $3.50(2.42-5.06)$ \\
\hline DAS-A perfectionism (score>38) & $0.48(0.17)$ & $8.27^{\star}$ & $1.61(1.16-2.23)$ \\
\hline QwQ extreme positive responses (score $>0.5$ ) & $-0.48(0.16)$ & $9.12^{\star}$ & $0.62(0.45-0.85)$ \\
\hline \multicolumn{4}{|l|}{ MDD 7-9 $(N=497)$ vs. MDD 5-6 $(N=361)^{\mathrm{b}}$} \\
\hline Gender (male) & $-0.27(0.16)$ & 2.68 & $0.75(0.41-1.03)$ \\
\hline Age (>42 years) & $0.10(0.16)$ & 0.39 & $1.17(0.85-1.60)$ \\
\hline Education (medium to high) & $-0.05(0.18)$ & 0.08 & $0.96(0.67-1.36)$ \\
\hline Employment (employed) & $-0.44(0.16)$ & $7.14^{*}$ & $0.62(0.45-0.86)$ \\
\hline DID interference (score>10) & $1.03(0.17)$ & $38.18^{\star \star}$ & $2.74(1.97-3.81)$ \\
\hline DID quality of life (score>21) & $0.88(0.17)$ & $26.84^{\star \star}$ & $2.31(1.65-3.23)$ \\
\hline QwQ extreme negative responses (score $>0.5$ ) & $0.54(0.17)$ & $9.70^{*}$ & $1.68(1.19-2.36)$ \\
\hline \multicolumn{4}{|c|}{ 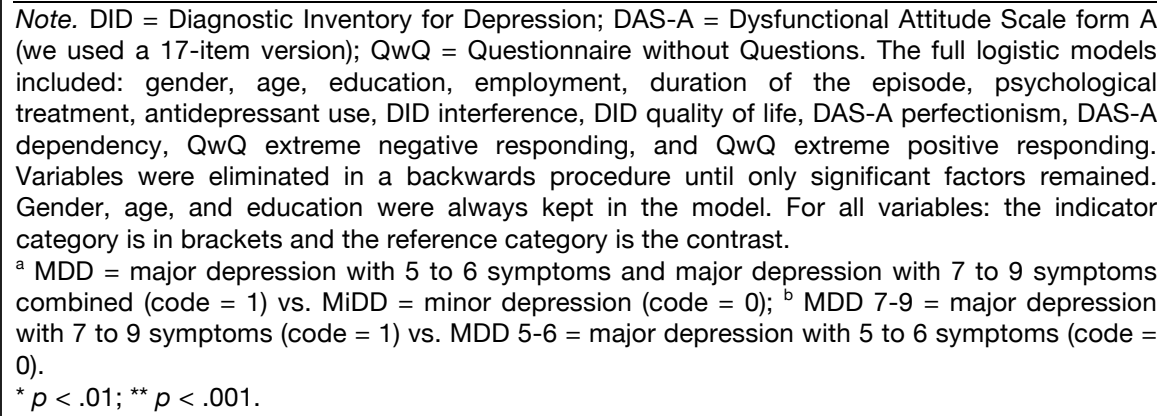 } \\
\hline
\end{tabular}




\section{Discussion}

\section{Main findings}

The present study was set up to extend previous work on minor depression using a large community sample. Demographic, clinical, and cognitive characteristics (i.e. content and form of dysfunctional thinking) were compared between minor depression (MiDD), major depression with 5 to 6 symptoms (MDD 5-6), and major depression with 7 to 9 symptoms (MDD 7-9). Age, education level, and having a partner did not distinguish minor depression and the two major depression categories, but gender and employment status did. Pronounced differences in terms of clinical characteristics and cognitive factors were found between the three depression status categories. In general, effect sizes for clinical features were larger than for cognitive variables, especially for MDD 7-9 compared to MDD 5-6. Correlations between number of symptoms and the clinical factors were moderate and larger than correlations with the cognitive factors. The logistic regression models showed that gender, employment status, interference, quality of life, dysfunctional attitudes, and extreme responding substantially distinguished major depression from minor depression.

\section{Continuum}

The hypothesis presented in the introduction was only partly confirmed by the current findings. Respondents in the three depression status categories did not differ with regard to age, education, and having a partner. Variables marking the severity were different across the three depression status categories and covaried linearly with the number of symptoms. Dysfunctional thinking showed the same pattern, which might also, in part, reflect depressive severity. Since these cognitive features were less directly related to the severity of the disorder than the clinical characteristics, smaller correlations and effect sizes were obtained. Taken together, these results seem to confirm previous results that minor depression is part of a continuum together with major depression (see Backenstrass et al., 2006; Cuijpers et al., 2004; Kessler et al., 1997; Prisciandaro \& Roberts, 2005).

However, the possibility that minor depression is in some aspects different from major depression cannot be ruled out. Compared with MiDD, men were less likely to suffer from MDD than women, which corresponds with results found in previous studies (Cuijpers et al., 2004; Kessler et al., 1997). In addition, employment status significantly differed between the three depression status categories. This may reflect the impairment associated with the more severe depression categories; probably more respondents are on sick leave when the severity of the depression increases. Some taxometric studies also found clear indications for a taxon, indicating that there is a major distinction between respondents with major depression and those without depressive complaints (e.g. Ruscio et al., 2007; Solomon et al., 2006).

It might be possible that that there is a continuum from mild to severe depression, but that depressed individuals are distinct from those who do not suffer from any depressive complaints at all. The use of a community sample, like in the present study, might provide a large diversity in clinical presentation of the depressive disorder as opposed to a subsample of depressed individuals who are recruited in clinical settings. Clearly, more research is needed before a firm conclusion about the continuum issue can be drawn. 


\section{Dysfunctional thinking: content and form}

Most research investigating dysfunctional thinking in depression has focused on major depression. However, such studies should take the underlying structure of depression into account, which can increase the statistical power (see also Ruscio et al., 2007). In the current study, a relatively strong contribution of dysfunctional thinking to the distinction between major and minor depression has been shown, especially for extreme responding. Some of the present findings regarding dysfunctional thinking in depression warrant some extra reflection.

Firstly, the results suggest a gradual increase in both form and content of dysfunctional thinking from MiDD to MDD 7-9. This is in line with some previous studies (Alloy et al., 2000; Alloy et al., 2006; Gibb et al., 2004). However, effect sizes were relatively small, suggesting that the cognitive factors are not particularly large contributors to the distinction between the three groups.

Secondly, perfectionism was more strongly associated with the more severe forms of depression than dependency. Previously, it has also been suggested that perfectionism covaries more closely with symptom state than dependency (Beck et al., 1991; Coyne \& Whiffen, 1995). Because of our cross-sectional design, dysfunctional thinking could not be distinguished from its manifestation in depression. Dysfunctional cognitive factors may therefore merely be manifestations of current symptom severity.

Thirdly, the form of dysfunctional thinking (i.e. extreme responding) is not only predictive of relapse or poor treatment outcome (see Beevers et al., 2003; Petersen et al., 2007; Teasdale et al., 2001), but is also associated with an increasing number of depressive symptoms as was shown in the current study. This suggests that the importance of the specific content of cognitions in major depression might need to be reconsidered. If these results can be replicated, this might also have implications for cognitive therapy. Next to seeking to modify the content of cognitions, which is classically the main feature of cognitive therapy, clinicians and researchers should also seek ways to reduce the extreme response style of depressed patients. This is in line with mindfulness based cognitive therapy, in which the main aim is to learn to decentre from negative thinking patterns (Teasdale et al., 2000).

Finally, the current findings are in contrast with the construct of 'dichotomous thinking', which refers to evaluating experiences in terms of exclusive categories either extremely negative or extremely positive. While extreme negative responding indeed increased across the three depression categories, extreme positive responding decreased in the current study. This might suggest that in depression there is simply a general tendency towards evaluating everything in life extremely negative, even senseless items on the Questionnaire without Questions. This might to some extent influence and, therefore, falsely elevate scores on questionnaires measuring cognitive content (e.g. DAS-A), resulting in an overestimation of their relation to depression. However, not until these findings have been replicated, one should remain tentative in making conclusions. Future research needs to focus more on the form of dysfunctional thinking.

\section{Clinical implications}

Minor depression is associated with significant impairment and risk of developing major depression (e.g. Cuijpers \& Smit, 2004). Since there is also a low likelihood of 
spontaneous remission in treatment-seeking samples in primary care (Hegel et al., 2006), qualified treatments are needed. Patients with minor depression may differ from patients with major depression in the kind of treatment that is needed. Patients with minor depression can be presented with the least intrusive interventions, only proceeding to more intense treatment when the complaints are more severe. In this stepped-care approach (e.g. see Scogin et al., 2003), knowledge on how to categorise patients into subgroups is essential. Since cut-off scores of questionnaires assessing depressive severity are arbitrary, other factors need to be taken into consideration, such as employment status and extreme responding that distinguished major depression from minor depression in the current study. In future research, factors that determine the boundaries between the different subgroups of depression need to be targeted. This may help to determine which treatments are suitable for whom.

\section{Methodological considerations}

The main strength of the current study is the large sample size $(N=1129)$, providing us with sufficient power to detect significant differences in the various depressive subgroups. Another strength is the use of a community sample. Unlike in clinical samples, no biases occurred due to help seeking behaviour of patients and illness recognition by physicians, which is a problem in depression (Paykel et al., 1997). A final strength is that, to our knowledge, we were the first to evaluate and compare both content and form of dysfunctional thinking in both minor and major depression.

Several limitations of the present study should also be noted. First, the full response rate was very low (8\%). Since no major discrepancies on demographic variables arose between our sample and the population in the Southern part of the Netherlands, this might not be a reason for concern. Moreover, selection bias is less of an issue here, since only depressed responders were selected and within-group analyses were conducted. Second, it should be noted that in the present study the ratio of minor and major depression is one to three, whereas previous studies reported higher prevalence rates for minor depression. Affinity with the subject might have been a reason for participation in the study. Third, since predominantly Caucasian individuals participated, future studies need to investigate samples representing different demographic and cultural backgrounds. Fourth, the cross-sectional design of our study does not allow investigating possible cause-effect relationships. To address this issue, experimental designs are needed. Fifth, the generalizability of the present findings might be limited due to the recruitment method. Only individuals with access to the Internet were able to participate. In line with this, one may question the equality of computerised questionnaires and paper-and-pen versions. However, there are sufficient indications that computerised and paper-and-pen questionnaires show similar construct validity (Butcher et al., 2004; Butcher et al., 2000). Sixth, selfreport measures were used. A diagnostic interview might more accurately categorise participants into minor depression, major depression, and severe major depression. Moreover, information on dysthymia and comorbidity is lacking. Finally, a new paradigm to measure extreme response styles (i.e. Questionnaires without Questions) in depression was used. Although we believe this is a better method than calculating the number of extreme responses on a depression related questionnaire, which might be confounded by depressive severity, future research should refine the methodology for measuring extreme responses (see also Petersen et al., 2007). 


\section{Conclusion}

In conclusion, the current findings predominantly confirm the results from earlier studies that minor depression is part of a continuum together with major depression. Both researchers and clinicians should, therefore, take the underlying structure of depression into account and focus more on subgroups along the continuum. Furthermore, not only clinical factors, but also dysfunctional cognitive factors, seem to act in synchrony with the depression continuum. Not only the specific content of dysfunctional thinking, also the form seems to play a major role in depression. Depressed patients have a general tendency towards extreme negative responding. If the current results can be replicated and extended, this may have important implications for the cognitive theory of depression. 


\section{Acknowledgements}

We thank Annie Hendriks, Greet Kellens, and Sylvia Gerhards for their assistance during the study and Rosanne Janssen for the development of the infrastructure for the online data-collection. The study was financed by ZonMw (Netherlands Organisation for Health Research and Development; project number 945-04-417), research institute EPP and research institute CAPHRI. Municipalities Eijsden, Meerssen, SittardGeleen, Valkenburg and Maastricht sponsored the study. 


\section{Chapter 4}

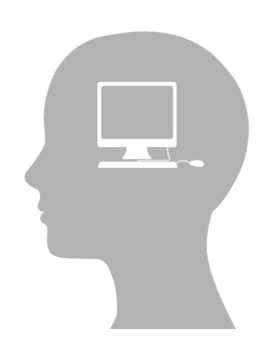

\section{Clinical and cost-effectiveness}

of computerised cognitive

\section{behavioural therapy for}

depression in primary care:

\section{design of a randomised trial}

L.E. de Graaf, S.A.H. Gerhards, S.M.A.A. Evers, A. Arntz, H. Riper,

J.L. Severens, G. Widdershoven, J.F.M. Metsemakers \& M.J.H. Huibers

This chapter is published in: BMC Public Health (2008), 8: 224. 


\section{Abstract}

Background: Major depression is a common mental health problem in the general population, associated with a substantial impact on quality of life and societal costs. However, many depressed patients in primary care do not receive the care they need. Reason for this is that pharmacotherapy is only effective in severely depressed patients and psychological treatments in primary care are scarce and costly. A more feasible treatment in primary care might be computerised cognitive behavioural therapy. This can be a self-help computer program based on the principles of cognitive behavioural therapy. Although previous studies suggest that computerised cognitive behavioural therapy is effective, more research is necessary. Therefore, the objective of the current study is to evaluate the (cost-)effectiveness of online computerised cognitive behavioural therapy for depression in primary care.

Methods/Design: In a randomised trial we will compare (a) computerised cognitive behavioural therapy with (b) treatment as usual by a GP, and (c) computerised cognitive behavioural therapy in combination with usual GP care. Three hundred mild to moderately depressed patients (aged 18-65) will be recruited in the general population by means of a large-scale Internet-based screening $(N=200,000)$. Patients will be randomly allocated to one of the three treatment groups. Primary outcome measure of the clinical evaluation is the severity of depression. Other outcomes include psychological distress, social functioning, and dysfunctional beliefs. The economic evaluation will be performed from a societal perspective, in which all costs will be related to clinical effectiveness and health-related quality of life. All outcome assessments will take place on the Internet at baseline, two, three, six, nine, and twelve months. Costs are measured on a monthly basis. A time horizon of one year will be used without long-term extrapolation of either costs or quality of life.

Discussion: Although computerised cognitive behavioural therapy is a promising treatment for depression in primary care, more research is needed. The effectiveness of online computerised cognitive behavioural therapy without support remains to be evaluated as well as the effects of computerised cognitive behavioural therapy in combination with usual GP care. Economic evaluation is also needed. Methodological strengths and weaknesses are discussed. 


\section{Background}

Major depression is a common mental health problem in the general population (Bijl et al., 1998) and it is reported to be the second most common and costly mental health problem in general practice (Stewart et al., 2003). Depression is associated with substantial decreases in quality of life through its impact on physical, social and emotional functioning, and well-being (Bijl \& Ravelli, 2000; Kruijshaar et al., 2003). By 2020 , depression is estimated to be the second leading contributor to the global burden of disease (Murray \& Lopez, 1997). Cost-of-illness studies reveal that the economic burden of depression is considerable (Cuijpers et al., 2007a; Luppa et al., 2007; Slobbe et al., 2006).

\section{Difficulties in the treatment of depression in primary care}

The general practitioner (GP) is the major health care provider involved in the primary care treatment of depression. In the Dutch health care system the GP is seen as a gatekeeper, and as a result patients view their GP as a key figure in the detection and treatment of depression (CBO, 2005). Despite this, many depressed patients remain undetected (Paykel et al., 1997; Spijker et al., 2001; Tiemens et al., 1996). Even when the depressive complaints are being recognised, many patients in primary care do not receive the care they need. There are several reasons for this. First, time for the management of psychosocial problems is lacking (van Schaik et al., 2002). Second, pharmacotherapy is only effective in extremely depressed patients (Kirsch et al., 2008), and many patients refuse medication or comply poorly (Simon et al., 1996). Third, effective non-pharmacological treatment options, such as psychotherapy, in primary care are scarce or not feasible (Bower et al., 2003). Consequently, only a limited group of depressed patients in primary care receives effective treatment.

\section{Computerised cognitive behavioural therapy in primary care}

Cognitive behavioural therapy (CBT) is one the most widely researched forms of psychotherapy. Cognitive behavioural therapy (CBT) has proven to be as effective as pharmacotherapy in the acute phase of mild to severe depression, and seems even more effective in the prevention of recurrence and relapse (Butler et al., 2006; Hollon et al., 2006; Vittengl et al., 2007). Despite its effectiveness, face-to-face CBT in primary care has some major limitations. There are not enough well trained therapists, it is costly, there are waiting lists, and patients may feel reluctant to enter psychotherapy. An alternative treatment in primary care might be computerised cognitive behavioural therapy (CCBT): a computer program based on the principles of CBT. The level of therapist support can vary considerably in CCBT. It can be offered as a self-help intervention without or with only minimal support. Previously, written self-help based on CBT seemed a promising treatment for depression (Cuijpers, 1997). In a primary care setting, positive outcomes were found regarding the (cost-) effectiveness of written self-help with minimal contact in subthreshold depression relative to care as usual provided by the GP (Smit et al., 2006; Willemse et al., 2004).

CCBT for primary care seems promising; it provides an acceptable alternative to pharmacotherapy, it can save clinicians' time, and the costs are low compared with face-to-face CBT. Furthermore, CCBT has a high accessibility, the number of referrals to secondary care by a GP can be reduced, and waiting lists for traditional CBT can become shorter (Kaltenthaler et al., 2006; Titov, 2007). Next to that, CCBT may fit 
very well in a stepped care program, and may function as a first step in the treatment of depression (Scogin et al., 2003). In a recent systematic review (Kaltenthaler et al., 2006), it was concluded that CCBT is a feasible, effective and acceptable treatment for depression. However, most research on the efficacy of CCBT has been conducted in the general population or within clinical or specialist settings. To our knowledge, only one study, so far, investigated the efficacy of CCBT for depression in primary care (Proudfoot et al., 2004), and it was shown that CCBT (delivered on a personal computer in the general practice) is more effective than usual care by a GP in mild to moderate depression and anxiety. Furthermore, this intervention seemed promising regarding the cost-effectiveness compared with usual GP care. CCBT was both more effective and more costly compared with usual GP care. When willing to pay for an additional unit of effect, CCBT could be very cost-effective. If a value of $£ 40$ is placed on a unit reduction on the Beck Depression Inventory, the probability of CCBT being cost-effective is in excess of $80 \%$. At a value of $£ 5000$ for 1 quality-adjusted life-year (QALY), the study showed that there is an $85 \%$ chance of CCBT being more cost-effective, and at a value of $£ 15000$ per QALY it exceeds a 99\% chance of being cost-effective (McCrone et al., 2004). Nevertheless, more research is necessary; so far only this one study has conducted an economic evaluation of CCBT, and the effects of CCBT in combination with usual care by a GP are still unknown. In addition the efficacy of CCBT via the Internet in primary care remains to be evaluated. The Internet can offer further advantages in comparison to CCBT on a stand-alone computer; it is easily accessible and it can be used at home, anonymously, and it is available 24/7.

\section{Current study}

In the present study we aim to evaluate the (cost-)effectiveness of online CCBT for mild to moderate depression in primary care. In a randomised trial we will compare (a) CCBT with (b) treatment as usual (TAU) by a GP, and (c) CCBT in combination with TAU. In a recent Dutch study of Spek et al. (2007b), the same CCBT program has shown to be equally effective as group CBT in people over 50 years old with subthreshold depression (Spek et al., 2007b). Based on the results from a recent systematic review (i.e. Kaltenthaler et al., 2006) we hypothesise that CCBT will be more effective than TAU by a GP. Furthermore, we hypothesise that CCBT in combination with TAU will be more effective than CCBT alone by increasing treatment adherence. Although some studies have shown that for mild depression a combination of pharmacotherapy and psychotherapy does not appear more effective than psychotherapy alone (Hirschfeld et al., 1997), other studies suggested that patients receiving combined treatments were more likely to stay in treatment and comply to the treatment protocol (Edlund et al., 2002).

Regarding the economic evaluation we hypothesise the following. The self-help intervention CCBT alone implies costs of time spent by the patient on the treatment and costs of the development of the program, while the TAU treatment requires costs related to a GP consult and/or medication. We hypothesise that from a societal perspective, these costs of the intervention CCBT are comparable to the costs of the intervention TAU. However, as a consequence of our hypothesis that CCBT is more effective than TAU, we expect that CCBT will be more preventive in health care use and productivity loss during the follow-up period, and thus result in lower costs compared with TAU by a GP. As a consequence of the expected increase of effectiveness 
(in terms of depression and quality of life) and decrease of costs, we hypothesise that CCBT is more cost-effective than TAU by a GP. Hypotheses about the intervention CCBT in combination with TAU are that it is both more costly and more effective compared with stand alone CCBT or TAU. 


\section{Methods}

\section{Design}

We will conduct a randomised controlled trial. Patients will be randomly allocated to one of the three following conditions: (a) CCBT, (b) TAU by a GP, and (c) a combination of CCBT and TAU by a GP. The design of the study and the anticipated flow of the participants are graphically shown in figure 1. The Medical and Ethical Committee of Maastricht University approved the study protocol. The study is registered at the Netherlands Trial Register, part of the Dutch Cochrane Centre (ISRCTN47481236).

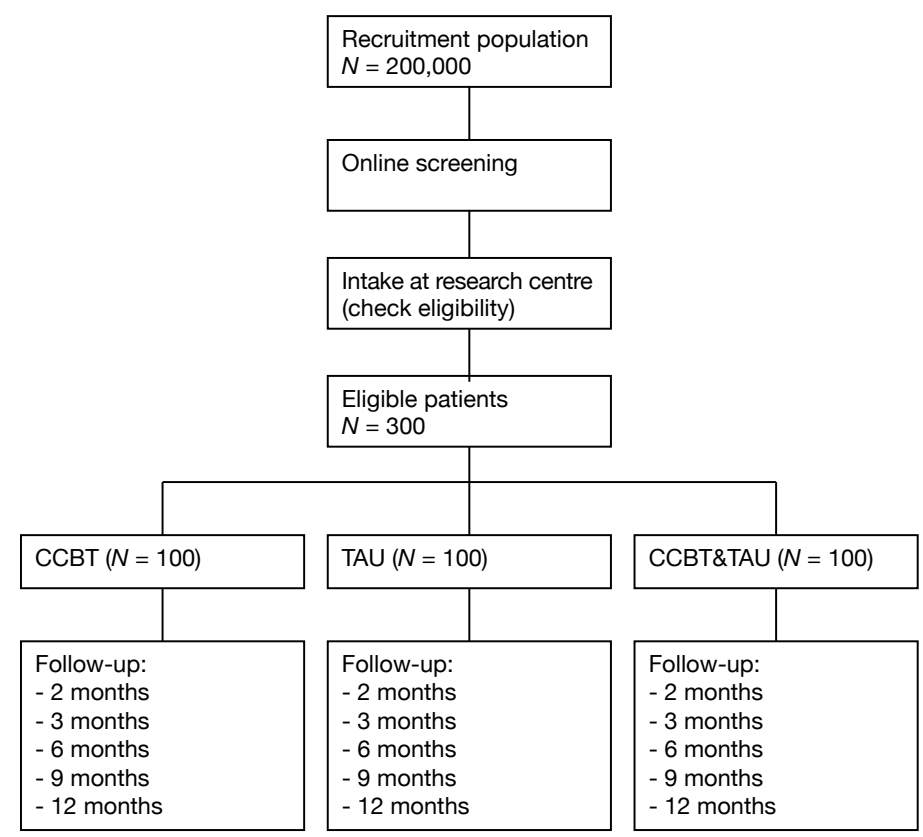

Figure 1 Flow of the participants

Note. CCBT = computerised cognitive behavioural therapy; TAU = treatment as usual by a GP.

\section{Study population}

The patient population we aim to investigate consists of 300 mild to moderately depressed adults. Patients are eligible to participate if they meet the following criteria: age 18 to 65 years; access to the Internet at home; at least mild to moderate depressive complaints (BDI-II score $\geq 16$ ), although a DSM-III-R diagnosis of major depression was not required; duration of depressive complaints 3 months or more; no current psychological treatment for depression; no continuous antidepressant treatment for 3 months or more prior to entry; fluent in Dutch language; no alcohol and/or drug dependence; and no severe psychiatric co-morbidity (e.g. psychotic disorders).

\section{Sample size}

Power calculations are based on elementary head-to-head comparisons of CCBT versus usual care and CCBT versus the combined treatment (t-test). A clinically rel- 
evant treatment effect is derived from the only study of CCBT in primary care (Proudfoot et al., 2004). For a mean difference in change scores of 5 (SD 5.25) on the BDI-II, a sample size of 84 participants per group is needed (power $90 \%$, alpha 0.05). Adjusting for potential study withdrawal (20\%), we estimate that 100 participants per group are needed.

\section{Recruitment}

Participants will be recruited in the general population by means of a large-scale Internet-based screening in the South of the Netherlands. A random selection of individuals $(N=200,000)$ will be sent an invitation letter to complete a screening questionnaire (i.e. BDI for primary care) via the Internet. The letters will be sent in weekly badges. Six municipalities cooperate by issuing names and addresses of their residents on a weekly base. The letters will contain information on the study protocol as well as log in codes for the questionnaires on the Internet. Participants who score above the cut-off score of four on the BDI for primary care (Steer et al., 1999) will be invited to visit the research centre for an interview where final eligibility will be assessed based on the in- and exclusion criteria. After inclusion we will notify the patients' GP about his or her participation in the study.

\section{Randomisation and procedure}

Randomisation will take place after informed consent is obtained. An independent statistician will develop the randomisation code. Block randomisation will be used to ascertain that each intervention consists of equally large groups. The randomisation code will be given to an independent IT-specialist who will develop a computer program to carry out the group allocation. On entry into the trial the computer program provides the next available number. The randomisation code will not be revealed until participant inclusion is complete. In view of the nature of the treatments, blinding of the participants and researchers is not possible.

Baseline assessment will take place at the research centre before randomisation. The questionnaires will be administered on a computer. All follow-up assessments will take place at home via the Internet at two, three, six, nine and twelve months after inclusion. Preceding an upcoming assessment point, participants will receive an e-mail alert. If a participant fails to complete the assessment within one week, an email-reminder will be sent. When the participant still fails to fill out the questionnaires, a phone call will be made.

Individuals will not be paid for participation, but will receive a small fee for Internet use.

\section{Interventions}

The CCBT program (named "Colour Your Life") is an online, multimedia, interactive, self-help computer program for depression, and was developed by Riper and Kramer (2004) of the Trimbos Institute (the Netherlands Institute of Mental Health and Addiction). The program is based on the principles of CBT and on the Dutch version of the 'coping with depression course' of Lewinsohn (Cuijpers et al., 1995; Lewinsohn et al., 1984). The program consists of eight weekly 30-minute sessions and a ninth booster session, although the duration of sessions can vary among users. At the end of each session homework-assignments are given, such as keeping a 'mood diary'. Patients 
allocated to the intervention CCBT or the combination CCBT and TAU will be given log in codes by the researchers at inclusion and they will access the program at home. No professional assistance will be offered. The program was originally developed for people over 50-years old (Spek et al., 2007b) and is adapted for an adult population (18 to 65 years) for the current study. GP's of patients allocated to CCBT only will be informed about the participation and treatment allocation of their patient.

In the TAU and CCBT\&TAU group, participants will be advised to contact their own GP. GPs of patients in the TAU and the CCBT\&TAU group will be sent a letter, informing them about their patients' study participation, and advising them to follow the depression guideline as described by the Dutch College of General Practitioners (Nederlands Huisartsen Genootschap [Dutch College of General Practitioners], 2003). This guideline states that treatment should formally consist of four to five biweekly consultations over the course of nine weeks in combination with antidepressant treatment if indicated. In case of suicidal risk, social dysfunction, symptom deterioration or non-improvement in six to twelve weeks time, referral to specialist mental health care settings is recommended. In practice however, usual care is whatever the GP prescribes.

An integrity check will be performed to assess treatment quality and protocol compliance using computer records of the CCBT program and questionnaires assessing use of GP and other health care (i.e. health care use questionnaire).

\section{Instruments}

Instruments will be used for the screening process, the clinical outcome assessment and the economic evaluation. In table 1 an overview of all assessments per time point is shown.

\section{Beck Depression Inventory for Primary Care}

The Beck Depression Inventory for Primary Care (BDI-PC) is a screening instrument for depression consisting of seven items derived from the Beck Depression Inventory II. Each item is assessed at a 4-point Likert-scale (range $0-4$ ). Several studies have proven its sound psychometric properties (Beck et al., 1997; Steer et al., 1999; Winter et al., 1999). A cut-off score of 4 was used in the current study, since this has excellent sensitivity and specificity for identifying a diagnosis of major depression (Steer et al., 1999).

\section{Composite International Diagnostic Interview}

To determine in- and exclusion criteria the computerised Composite International Diagnostic Interview (CIDI-auto) will be used. The CIDI is an extensive, fully structured diagnostic interview to assess lifetime and 12-month DSM-III-R diagnoses. The CIDI can be used by lay-interviewers and has acceptable reliability and validity (Robins et al., 1988; Wittchen, 1994).

\section{Beck Depression Inventory Second Edition}

The Beck Depression Inventory II (BDI-II) will be used to measure depressive severity. The total score is the sum of the 21 items with a range of 0 (no depression) to 63 (severe depression). There has been consistent support for the construct validity and reliability of the BDI-II in various samples (Arnau et al., 2001; Beck et al., 1996; van der Does, 2002). 


\section{Functional impairment}

Functional impairment will be assessed with the SF-36 Health Survey (SF-36), which consists of 36 items measuring 8 multi-item scales. For each subscale items are coded, summed, and transformed on to a scale from 0 (worst possible health state measured by the questionnaire) to 100 (best possible health state). The SF-36 has good psychometric properties in terms of validity, reliability, and scale structure (Aaronson et al., 1998; McHorney et al., 1993; Ware \& Sherbourne, 1992). Additionally, the economic evaluation will use the SF-6D utility, which is a quality of life measure derived from the SF-36 (Brazier et al., 2002).

\section{Psychological distress}

The Symptom Checklist (SCL-90) is a self-report symptom inventory of psychopathology. The SCL-90 consists of 90 items, each rated on a five-point scale of distress from 'not at all' to 'extremely' (Arrindell \& Ettema, 1981; Derogatis et al., 1976). The total score of the SCL-90 can be used as an index of severity for general psychological distress (score range 90-450) (Koeter et al., 1988). The SCL-90 has a high degree of reliability and support has been found for the validity (Arrindell \& Ettema, 1981).

\section{Social functioning}

The Work and Social Adjustment Scale (WSAS) is a self-report scale of functional impairment attributable to an identified problem. The WSAS consists of five items measured on an 8-point Likert-scale (0 to 8). A high score indicates severe impairment. It has good psychometric properties (Mundt et al., 2002).

\section{Dysfunctional attitudes}

The Dysfunctional Attitude Scale form A (DAS-A) is a self-report scale designed to measure the presence and intensity of dysfunctional attitudes. The DAS-A consists of 40 items and each item consists of a statement and a 7-point Likert-scale ( 7 = fully agree; 1 = fully disagree). The higher the score, the more dysfunctional attitudes an individual reports (Weissman \& Beck, 1978).

\section{Quality of life measures for the economic evaluation}

Measuring health-related quality of life is relevant in patients with depression, since depression has a large impact on the physical, social and emotional aspects that are relevant and important to a patient's well-being (Bijl \& Ravelli, 2000; Kruijshaar et al., 2003). An intervention aimed at treating depression is therefore expected to have an impact on the perceived quality of life. In addition, measuring generic quality of life facilitates the comparison of the effects on quality of life of our intervention program with that of other interventions (Drummond et al., 2005). Quality of life will be measured in three different ways in this study by using the WHOQOL-BREF, the EuroQol and the SF-6D.

The WHOQOL includes a broader range of mental health aspects in its psychological domain compared to other quality of life instruments and is therefore more feasible to use in this study (Orley et al., 1998). The WHOQOL-BREF is an abbreviated version of the WHOQOL-100, which has proven to be a valid and reliable alternative (Trompenaars et al., 2005; WHOQOL-Group, 1998). The WHOQOL-BREF measures four domains related to quality of life (physical health, psychological health, social re- 
lationships and environment) and includes two questions on overall quality of life and general health (WHOQOL-Group, 1998).

Generic quality of life will be derived by means of the EQ-5D of the EuroQol group. The EQ-5D consists of five health state dimensions (mobility, self-care, usual activity, pain/discomfort and anxiety/depression) on which the respondent has to indicate his own health state (Brooks, 1996; EuroQol Group, 1990). The EuroQol will be assessed since it is a validated and widely used quality of life instrument, both nationally and internationally (Szende et al., 2007). An advantage of the EuroQol is that it is short and that an overall utility score for population-based quality of life can be obtained, which facilitates comparisons with other interventions and health states in other disease areas. A utility refers to the preference that individuals or society may have for any particular set of health outcomes. It is indicated by a number between 0 (the worst imaginable condition: death) and 1 (perfect health) (Drummond et al., 2005). Standardised value sets are available to calculate the utility based on the EQ-5D. This study will use the Dutch tariff and the original UK tariff to value generic quality of life (Dolan, 1997; Lamers et al., 2006; Szende et al., 2007). The utility scores of the EQ-5D will be used to calculate the quality adjusted life year (QALY) during the follow-up period by adjusting the length of time affected through the health outcome by the utility value (Drummond et al., 2005).

An adapted version of the EuroQol is the $E Q-5 D+C$, in which a sixth domain (cognitive functions referring to memory, concentration, coherence and IQ-level) is added to the five existing domains of the EQ-5D (Hoeymans et al., 2005; Krabbe et al., 1999; Stouthard et al., 1997). This sixth domain of the EQ-5D+C is also included in the questionnaire to provide additional information on quality of life. However, since there is no tariff developed to compute utility scores for the $E Q-5 D+C$, utility scores and QALYs will only be calculated based on the five domains of the EQ-5D.

The SF-6D is a utility instrument based on the health-related quality of life questionnaire SF-36. The utility score is derived from 11 items of the SF-36 and is composed of six dimensions of health (physical functioning, role limitations, social functioning, pain, mental health, and vitality). The SF-6D utilities will be derived by means of the preference-based UK tariff (Brazier et al., 2002).

\section{Costs}

Costs will be defined from the societal perspective. Within our study we distinguish three cost-categories: health care sector costs, costs for the patient and family, and productivity costs (Drummond et al., 2005).

A health care use questionnaire will be developed to measure the psychological, paramedical, medical, paid and informal care, participation in a self-help group, and alternative treatments received by the patient on a monthly base. This health care use questionnaire will be based on an existing cost diary (Goossens et al., 2000) and retrospective cost questionnaires (Hakkaart-van Roijen, 2002; van Asselt et al., 2008), and is adapted to depressive patients. This questionnaire will provide information to calculate health care sector costs by measuring the volume of care provided, and out-of-pocket expenses, which are part of the costs for the patient and family. The other part of the patient and family costs will concern costs of travelling and lost time due to the primary care intervention TAU and/or CCBT. The time spent by a patient on CCBT will be tracked by means of the computer-registered login and logout data 
of the program. In the health care use questionnaire the duration of a GP consult will be registered by the patient. The number of GP consults informs on the number of travels from/to the GP, and will be linked to average distances from/to a GP. Dutch guideline prices will be used to value the costs of the health care items (Oostenbrink et al., 2004). If for specific cost-categories cost guidelines are unavailable, average tariffs or shadow prices will be used. The standard cost prices and tariffs of health care practitioners include the integral costs, being all costs directly and indirectly attributable to the cost unit.

For the measurement of production losses, the patient modules $B, C, D$ and E of the PROductivity and DISease Questionnaire (PRODISQ) will be used (Koopmanschap et al., 2004; Koopmanschap, 2005). These modules consist of questions concerning the profession, working situation, income, absence from work, compensation mechanisms in case of absence for paid work and productivity costs at work (efficiency loss) of the patient. Productivity costs will be calculated according to the friction cost approach (Koopmanschap, 2005; Oostenbrink et al., 2004), using one general cost price per lost hour of productivity (Oostenbrink et al., 2004). 


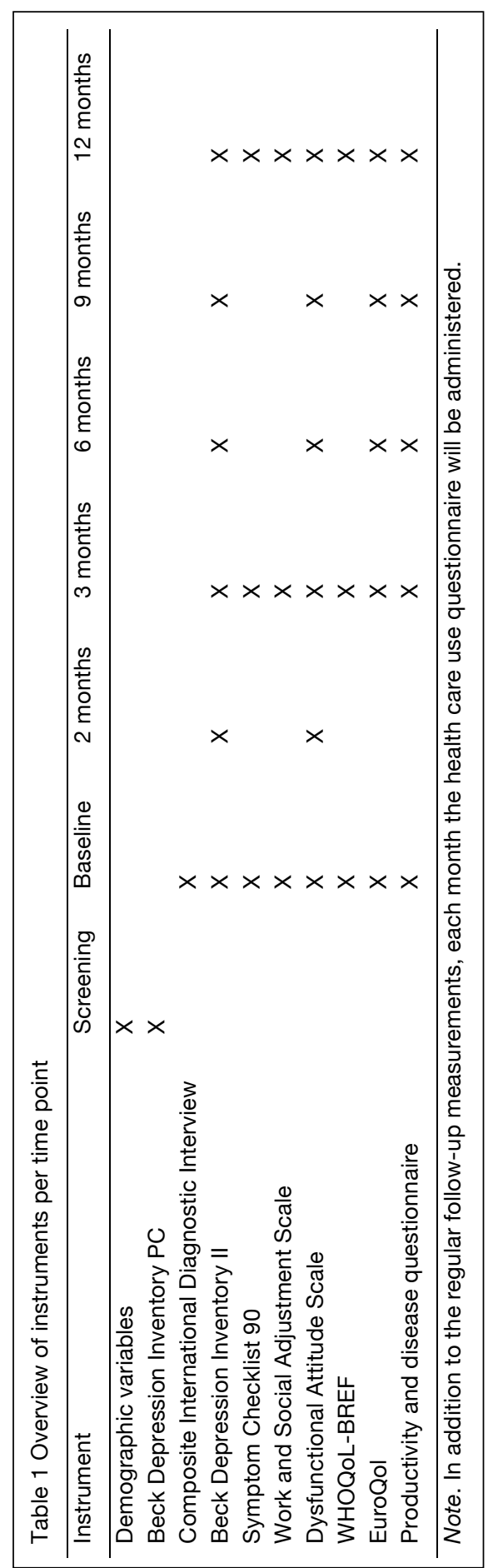




\section{Analyses}

\section{Clinical analyses}

Data-analysis will include intention-to-treat analysis and per-protocol analysis. Analysis will include elementary head-to-head comparisons of the intervention groups as well as more complicated multi-level analysis incorporating patients and time measurements if necessary. An integrity check and a process evaluation will be performed using qualitative methods of analysis. In case of missing data, we will impute intermittent missing data using the mean of the values of a previous and a subsequent time point. Other missing data (i.e. missing values due to lost to follow-up) will only be imputed when more then $15 \%$ of the data are missing.

To test the main hypotheses, difference scores for all outcome variables will be calculated $\left(t_{0}\right.$ minus $\left.t_{k}\right)$ and compared between the three groups using ANOVA. In case of violation of assumptions, robust ANOVA can be used (Wilcox, 2005). We will then compute improvement effect sizes and between-group effect sizes (Cohen, 1988) or robust equivalents (Algina et al., 2005). Next, we will calculate the number of patients who showed reliable and clinically significant change on the BDI-II using the method of Jacobson and Truax (1991). This calculation is based on two components: (1) the extent to which the pre-to-post-difference score is reliable taking into account the measurement variability of the instrument (reliable change; RC), and (2) the extent to which post-treatments scores are clinically meaningful (clinically significant change; CSC) (Evans et al., 1998). Chi-square tests will be used to test the frequency differences in $\mathrm{RC}, \mathrm{CSC}$ and $\mathrm{RC}+\mathrm{CSC}$ between the three groups.

\section{Economic evaluation}

Since the follow-up period lasts one year and no extrapolation over time will be executed, discounting of costs is not necessary. All costs will be indexed to the year 2007 by means of the price indexes of the Dutch Central Bureau of Statistics (CBS).

For each patient, volumes of care, travels, lost time for receiving care and lost productivity hours will be multiplied by the prices determined for each cost item. Based on the costs per item, costs during the follow-up period will be calculated as the cumulative costs per patient. The costs at baseline and during the follow-up period of the three groups will be compared by the non-parametric bootstrapping method with confidence intervals in percentiles. By bootstrapping, samples of the same size as the original data are drawn with replacement from the observed data (Briggs et al., 1997). The quality of life and clinical outcome variables will be compared between the three groups at baseline and during the follow-up period using ANOVA.

In case of baseline differences of costs, effectiveness, or utility scores between the patient groups, corrections will be performed (Brunenberg et al., 2005; Manca et al., 2005; van Asselt et al., 2009). The economic evaluation will consist of a base-case cost-effectiveness and cost-utility analysis, and sensitivity analyses. Uncertainty of parameter estimates of the base-cases will be dealt with by these sensitivity analyses (Drummond et al., 2005). In the base-cases, data will be analysed according to the intention-to-treat principle. Incremental cost-effectiveness ratios (ICERs) will be determined on the basis of incremental costs and incremental effects (Drummond et al., 2005) of (a) stand alone CCBT compared with (b) usual GP care, or (c) a combination of CCBT and usual GP care. The primary outcome measure for the cost-effectiveness analysis is depression measured by the BDI-II, and for the cost-utility analysis the 
QALY based on the EQ-5D. The cost-effectiveness ratio will be stated in terms of costs per point improvement on the BDI-II, the cost-utility ratio will focus on the net cost per QALY gained. In our primary analysis QALYs will be derived from the EQ-5D using the UK-tariff. Scores on the quality of life domains derived from the WHOQOLBREF and the sixth domain of the EQ-5D+C will be used to provide in-depth insight into the quality-of-life.

Non-parametric bootstrap re-sampling techniques will be used to explore uncertainty around estimates of cost-effectiveness derived from the study sample (Briggs et al., 1997). Decision uncertainty will be represented graphically by means of a costeffectiveness acceptability curve (CEAC) (Briggs, 2001; Drummond et al., 2005; Fenwick et al., 2004). In addition, the net monetary benefit (NMB) will be used to present the cost-effectiveness and cost-utility results in monetary units. The NMB expresses the difference in effects between the intervention groups in monetary values using the threshold willingness-to-pay for a unit of effect, minus the difference in costs between the interventions (Drummond et al., 2005; Stinnett \& Mullahy, 1998). Since the value that society would place on a unit reduction in BDI-II depression score is unknown, different values will be assumed to calculate the NMB (McCrone et al., 2004). Regarding the QALY, the Dutch Council of Public Health and Care suggested in 2006 a ceiling of $€ 80.000$ per QALY per year (Raad voor de Volksgezondheid en Zorg [Council for Public Health and Health Care], 2006). Alternative values, ranging to $€ 80.000$ per QALY, will be used to estimate the NMB.

The alternative threshold values of the NMB will be analysed in sensitivity analyses. Other aspects that can be part of a sensitivity analysis are: varying the utility outcome measure by using the SF-6D or alternative tariffs to value the EQ-5D utility (Dutch tariff instead of the UK tariff), or including other effectiveness measures used in the clinical evaluation. Cost prices will be varied as minimum and maximum cost price estimates. Next to the intention-to-treat analysis, a per-protocol analysis can be performed.

\section{Collaboration}

The current study will be conducted in collaboration with several disciplines. The Trimbos Institute (the Netherlands Institute of Mental Health and Addiction) will be involved in the development and dissemination of CCBT. The Pandora foundation (patient organization) acts as an advisor on the design of the study and the dissemination of the results. Several members of the project group work as clinicians in secondary mental health care institutions and have substantial professional contacts in the field. The Dutch College of General Practitioners (NHG) has been informed about our plans and supports our initiative. Additional research projects will be conducted in collaboration with the Department of Health, Ethics and Society/Metamedica and the Department of Health Organization, Policy and Economics of the Maastricht University. For instance, these projects will focus on topics like the patient perspective on CCBT and quality of life aspects of depression. 


\section{Discussion}

Although in the last two decades research attention for CCBT has grown, research on the effectiveness of CCBT for depression in primary care is still in its infancy, especially CCBT offered via the Internet. More research evaluating such interventions is necessary. Therefore, in the current study we will evaluate the (cost-)effectiveness of an online CCBT self-help program for mild to moderate depression in primary care. We will compare CCBT with treatment as usual by a GP, and with a combination of both treatments.

\section{Why do we need more research on CCBT in primary care?}

There are several reasons why we are conducting this study. First, the only study so far on CCBT in primary care used a program that was delivered on a computer in the general practice (Proudfoot et al., 2004). Since the Internet can increase the accessibility of such an intervention, we will offer the CCBT program on the Internet. In the Netherlands, almost all of the general adult population has access to Internet, and this will only increase in the next decade (Statistics Netherlands, www.cbs.nl). Second, in the only other study on CCBT in primary care (Proudfoot et al., 2004), a nurse provided practical support at the start and end of each session. We will study the effectiveness of CCBT as a pure self-help intervention; no support or guidance will be given to the patient. Third, the effects of a combined treatment (i.e. both CCBT and treatment as usual by a GP) still need to be evaluated. We think this might have extra effects in terms of improvement in depressive severity and quality of life. Although the GP is not directly involved in the CCBT program, a combined treatment might also increase adherence to the intervention. Previous studies already showed that minimal therapist contact could increase the adherence to Internet-based interventions (Andersson et al., 2005; Christensen et al., 2004a), a result which was recently confirmed in a metaanalysis (Spek et al., 2007a). Next to that, the GP can monitor the progress of the patient and can pay attention to non-verbal signals of the patient. Finally, only one study so far has conducted an economic evaluation of CCBT (McCrone et al., 2004).

\section{Methodological considerations}

Our study has several strengths. First, we will recruit patients from the general population. Unlike in samples selected in general practices or clinics, no biases will occur due to help seeking behaviour of patients and illness recognition by physicians, which is often a problem in depression (Paykel et al., 1997). Another strength is that we will make full use of the Internet infrastructure by administering all questionnaires online. This reduces the risk of making mistakes while filling out or scoring the questionnaires.

Several limitations of the present study should also be noted. All our outcomes will be measured online and one may question the equality of computerised questionnaires and paper-and-pen versions. However, there are sufficient indications that computerised and paper-and-pen questionnaires show similar construct validity (Butcher et al., 2004; Butcher et al., 2000). Furthermore, all the outcomes at follow-up will be measured by self-report and as a result information on actual DSM-III diagnoses of depressive episodes at follow-up will be lacking. 


\section{Conclusion}

CCBT is a new treatment format with interesting possibilities. It might offer a solution to the current undertreatment of depression. It is a promising treatment for depression in primary care, but more research needs to be done before it can be disseminated and implemented in the current health care system. The current study contributes to the growing literature on the clinical and cost-effectiveness of online CCBT. 


\section{Acknowledgements}

We thank Annie Hendriks and Greet Kellens for their assistance during the study. Rosanne Janssen develops the infrastructure for the online data-collection. The Pandora foundation has a consultative role. The trial is financed by ZonMw (Netherlands Organisation for Health Research and Development; project number 945-04-417), research institute Experimental Psychopathology (EPP) and the Care And Public Health Research Institute (CAPHRI). Municipalities Eijsden, Meerssen, Sittard-Geleen, Valkenburg, and Maastricht sponsor the study. 



\section{Chapter 5}

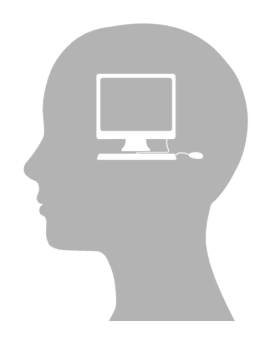

\section{Clinical effectiveness of online}

computerised cognitive

\section{behavioural therapy without}

support for depression in

\section{primary care: a randomised trial}

L.E. de Graaf, S.A.H. Gerhards, A. Arntz, H. Riper, J.F.M. Metsemakers,

S.M.A.A. Evers, J.L. Severens, G. Widdershoven \& M.J.H. Huibers 


\section{Abstract}

Background: Computerised cognitive behavioural therapy (CCBT) might offer a solution to the current undertreatment of depression.

Aims: To determine the clinical effectiveness of online, unsupported CCBT for depression in primary care.

Method: 303 depressed patients were randomly allocated to (a) Colour Your Life (CYL), (b) treatment as usual (TAU) by a GP, or (c) CYL and TAU combined. CYL is an online, multimedia, interactive CCBT program. No assistance was offered. We had a 6-month follow-up period.

Results: No significant differences in outcome between the three interventions were found in the intention-to-treat and per-protocol analyses.

Conclusion: Online, unsupported CCBT did not outperform usual care, and the combination of both did not have additional effects. Decrease in depressive symptoms in moderately to severely depressed patients was moderate in all three interventions. Online CCBT without support is not beneficial for all depressed patients. 


\section{Background}

Although cognitive behavioural therapy (CBT) is an effective treatment for depression (Butler et al., 2006; Hollon et al., 2006), many depressed patients in primary care remain untreated (Hirschfeld et al., 1997). An effective, acceptable, and feasible solution might be computerised CBT (CCBT) (Kaltenthaler et al., 2006). CCBT programmes can vary greatly in terms of technologies used and amount of additional support. To our knowledge, only one study so far investigated the efficacy of CCBT for depression in primary care (Proudfoot et al., 2004). It was shown that CCBT (delivered on a computer in the general practice) is more effective than usual GP care. Nevertheless, the effectiveness of CCBT via the Internet in primary care remains to be evaluated as well as the effects of CCBT combined with usual GP care. In a randomised trial, we addressed these issues by examining the effectiveness of online, unsupported CCBT (i.e. Colour Your Life) for depression in primary care. In another study, this intervention was equally effective as group CBT in people over 50 years old with subthreshold depression (Spek et al., 2007b). We hypothesise that CCBT will be superior to usual GP care, and that the combination of CCBT and usual GP care will be more effective than CCBT alone. 


\section{Method}

\section{Study population and recruitment}

Participants were recruited in the general population by means of a large-scale Internet-based screening in the South of the Netherlands. A random selection of individuals was sent an invitation letter to complete an online screening questionnaire. Potentially eligible participants were invited to visit the research centre to assess final eligibility. Participants were eligible if they met the following criteria: age 18 to 65 ; access to the Internet at home; at least mild to moderate depressive complaints (BDI-II score $\geq 16$ ); duration of depressive complaints 3 months or more; no current psychological treatment for depression; no continuous antidepressant treatment for at least 3 months prior to entry; fluent in Dutch language; no alcohol and/or drug dependence; and no severe psychiatric co-morbidity. To determine DSM-III-R axis I diagnoses the computerised Composite International Diagnostic Interview (CIDI-auto) (Robins et al., 1988) was used. Full details of the study method have been described elsewhere (de Graaf et al., 2008a).

\section{Procedure}

After informed consent was obtained, participants were randomly allocated to: (1) online CCBT without support (i.e. Colour Your Life), (2) treatment as usual (TAU) by a GP, (3) and online, unsupported CCBT and TAU combined. Baseline assessment took place at the research centre before randomisation on a computer. All follow-up assessments took place at home via the Internet. Preceding an upcoming assessment point, participants received an e-mail alert. Individuals received financial compensation for Internet use (i.e. €25).

\section{Interventions}

The CCBT program, named Colour Your Life (CYL) (Riper \& Kramer, 2004), is an online, multimedia, interactive computer program for depression. CYL is based on the principles of CBT and on the Dutch version of the 'coping with depression course' of Lewinsohn (Cuijpers et al., 1995; Lewinsohn et al., 1984). CYL consists of eight 30-minute sessions and a ninth booster session, although the duration of sessions can vary among users. At the end of each session homework-assignments are given. Participants were advised to complete one session per week. Participants were given log in codes by the researchers at inclusion and they accessed CYL at home. No assistance was offered. CYL was originally developed for people over 50-years old (Spek et al., 2007b) and was adapted for an adult population (18 to 65 years) for the current study.

TAU was delivered by the patient's own GP who was advised to follow the guideline described by the Dutch College of General Practitioners (van Marwijk et al., 2003). TAU can include four to five biweekly consultations and antidepressant treatment if indicated.

For each patient, it was assessed whether the interventions received were according to 'protocol'. Adherence to CYL was defined as being exposed to all essential steps of the intervention, which was operationalised as having completed five or more sessions. TAU adherence was defined as receiving at least four consultations or antidepressant medication. CYL\&TAU adherence was defined as a combination of both. 


\section{Outcomes}

The primary outcome measure was the severity of depression as measured with the Beck Depression Inventory Second Edition (BDI-II), high scores indicating severe depression (range 0-63) (Arnau et al., 2001; Beck et al., 1996; van der Does, 2002).

Secondary outcomes included the following measures. General psychological distress was measured with the Symptom Check-List 90 (SCL-90). Scores range from 90 (no distress) to 450 (very severe distress) (Arrindell \& Ettema, 1981; Derogatis et al., 1976). The Work and Social Adjustment Scale (WSAS) was used to assess impairment in social functioning attributable to depression (Mundt et al., 2002). A high score is indicative of severe impairment (score range 0-40). The 36-item short-form Health Survey (SF-36) was used to assess specific features of quality of life (Aaronson et al., 1998; McHorney et al., 1993; Ware \& Sherbourne, 1992). We used the two most relevant subscales: role limitations caused by emotional problems and general mental health. High scores indicate high levels of quality of life (score range 0-100). The intensity of dysfunctional beliefs was assessed with the Dysfunctional Attitude Scale form A (DAS-A) (Weissman \& Beck, 1978). We used a 17-item version (de Graaf et al., in press-b), with a score range of 17 to 119 . The higher the score, the more dysfunctional attitudes an individual reports.

Additional measures included a self-developed health care use questionnaire, which measured self-reported use of GP care, antidepressant medication, and specialist care.

All outcomes were assessed at baseline and at three months of follow-up. In addition, the BDI-II and the DAS-A-17 were also assessed at two months and six months of follow-up. The health care use questionnaire was assessed monthly.

\section{Sample size}

Power calculations were based on elementary head-to-head comparisons of CCBT versus usual care and CCBT versus combination treatment (t-test). We calculated that a sample size of 84 participants per group was needed to detect a change score of 5 (SD 5.25) on the BDI-II (power $90 \%$, alpha 0.05). Adjusting for potential study withdrawal (20\%), we estimated that 100 participants per group were needed.

\section{Data analysis}

Preliminary tests for distribution and outliers were performed. Skewness and Kurtosis did not indicate substantial deviations from normality for all outcomes. The analyses were based on the intention-to-treat principle (i.e. those who provided follow-up data irrespective of treatment-adherence). Only intermittent missing data were imputed ( $N$ $=5$ ) by calculating the mean of the values of a previous and a subsequent time point. Missing data due to lost to follow-up were not replaced by imputed values. We tested all effects at the $p<.05$ level (two-tailed). All analyses were carried out using SPSS (version 15.0.1 for Windows).

First, to test the main hypotheses, repeated-measures analyses of variance (ANOVAs) were performed. In case of significant time by group interactions, contrasts were conducted comparing changes from baseline to each subsequent time point for each pair of groups separately. Then, we computed improvement effect sizes for BDI-Il scores for each time point according to Cohen's d statistic (Cohen, 1988), defined as $\left(M t_{0}-M t_{k}\right) / S D_{(M t 0-M t k)}$. Between group effect sizes were determined by 
calculating the difference in improvement effect sizes between two groups. Next, we determined the proportion of patients who made clinically meaningful changes on the BDI-II using the methodology of Jacobson and Truax (1991). This approach is based on two components: (1) reliable change ( $R C)$, i.e. a decrease of 9 points, and (2) clinically significant change (CSC), i.e. a score below 12. Chi-square tests were used to test the frequency differences in RC, CSC, and RS+CSC between the three groups. Based on the RC+CSC proportions, the number needed to treat (NNT) was calculated (Cook \& Sackett, 1995). Finally, we conducted per-protocol analyses for treatment adherers only, using repeated measures ANOVA's for the BDI-II scores. 


\section{Results}

\section{Flowchart and participants}

Figure 1 presents the flow of the participants. Recruitment took place from December 2005 to June 2007. Follow-up ended December 2007. 303 depressed participants were enrolled in the study. At six months of follow-up, data were available for 275 participants (attrition rate 9.2\%). Reasons for lost-to-follow up were: too time consuming $(N=8)$, personal circumstances or medical illness other then a mental disorder $(N=$ $5)$, and no reason was given $(N=15)$. There seemed to be no baseline differences between participants who completed all assessments and those who were lost-tofollow-up (lowest $p$-value $=.10$ ). Baseline characteristics of all participants are shown in table 1, stratified according to intervention group. Randomisation was successful; the characteristics are fairly similar in all three groups, although gender did not seem equally distributed across the groups.

\begin{tabular}{|c|c|c|c|c|}
\hline \multicolumn{5}{|c|}{ Table 1 Baseline characteristics of the total sample } \\
\hline \multicolumn{2}{|l|}{ Variable } & $\begin{array}{l}C Y L \\
(N=100)\end{array}$ & $\begin{array}{l}\text { TAU } \\
(N=103)\end{array}$ & $\begin{array}{l}\text { CYL\&TAU } \\
(N=100)\end{array}$ \\
\hline \multicolumn{2}{|l|}{ Male sex, $\mathrm{nr}(\%)$} & $48(48.0)$ & $46(44.7)$ & $37(37.0)$ \\
\hline \multicolumn{2}{|c|}{ Age (18 -65), $M(S D)$} & $44.3(11.8)$ & 45.1 (12.2) & $45.2(10.9)$ \\
\hline \multirow[t]{3}{*}{ Education ${ }^{\mathrm{a}}$ : } & Low, nr (\%) & 18 (18.6) & $16(16.2)$ & $17(17.5)$ \\
\hline & Medium, nr (\%) & $55(56.7)$ & $55(55.6)$ & $52(53.6)$ \\
\hline & High, nr (\%) & $24(24.7)$ & $28(28.3)$ & $28(28.9)$ \\
\hline \multicolumn{2}{|c|}{ Partner, yes, $\mathrm{nr}(\%)^{\mathrm{b}}$} & $72(73.5)$ & $73(72.3)$ & $71(73.2)$ \\
\hline \multicolumn{2}{|c|}{ Employed, yes, $\mathrm{nr}(\%)^{\mathrm{c}}$} & 67 (72.0) & 64 (76.2) & 69 (73.4) \\
\hline \multirow{3}{*}{$\begin{array}{l}\text { Major depressive } \\
\text { episode: }\end{array}$} & No, $\mathrm{nr}(\%)$ & $22(22.0)$ & $18(17.5)$ & $21(21.0)$ \\
\hline & First, $\mathrm{nr}(\%)$ & $48(48.0)$ & $45(43.7)$ & $39(39.0)$ \\
\hline & Recurrent, nr (\%) & $30(30.0)$ & $40(38.8)$ & $40(40.0)$ \\
\hline \multicolumn{5}{|c|}{$\begin{array}{l}\text { Note. } \mathrm{CYL}=\text { Colour Your Life (CCBT offered online without support); TAU = treatment as } \\
\text { usual. } \\
\text { a data unavailable: CYL }(N=3), \operatorname{TAU}(N=4) \text {, and CYL\&TAU }(N=3) ;{ }^{b} \text { data unavailable: CYL } \\
(N=2), \text { TAU }(N=2) \text {, and CYL\&TAU }(N=3) ;{ }^{c} \text { data unavailable: CYL }(N=7) \text {, TAU }(N=17) \text {, } \\
\text { and CYL\&TAU }(N=6) \text {. }\end{array}$} \\
\hline
\end{tabular}




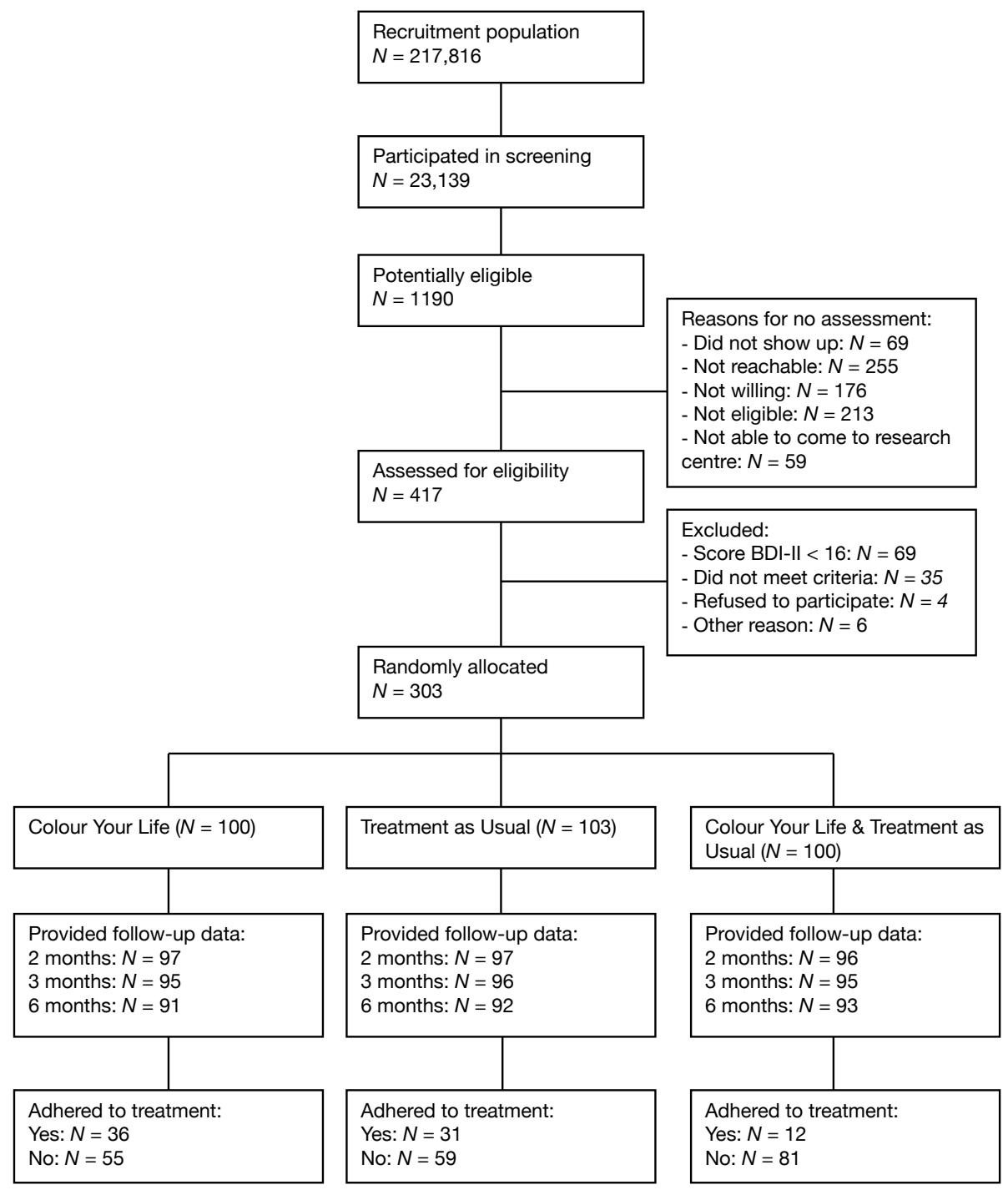

Figure 1 Flow of the participants 


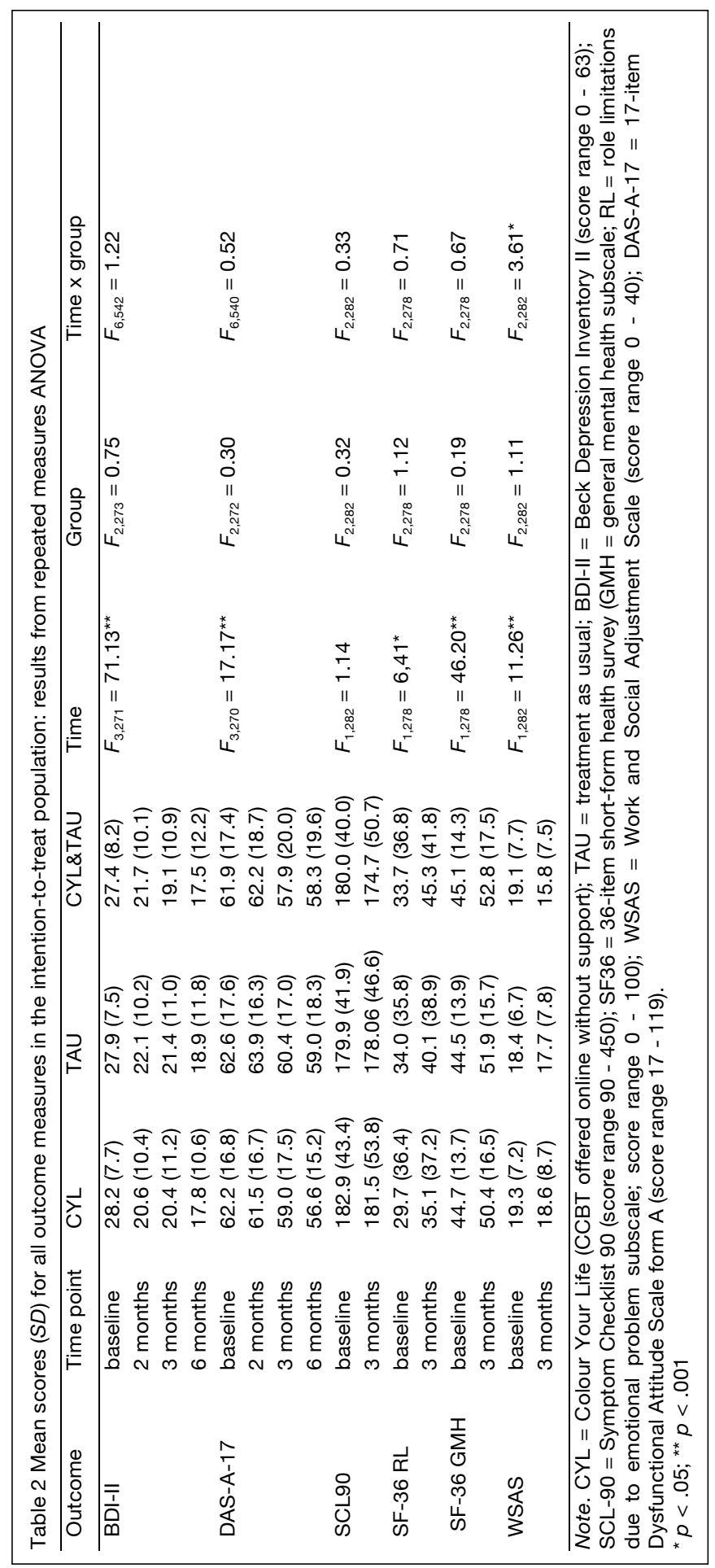




\section{Outcome of the interventions}

Table 2 depicts the means and standard deviations of the clinical outcomes at followup. There were no significant group by time interactions on the primary outcome measure as well as most secondary outcomes (all $p>$.29). Only a significant interaction effect was found for the WSAS $(p=.03)$. Contrasts revealed that CYL\&TAU resulted in a significant greater reduction on the WSAS compared to CYL alone $\left(F_{1,188}=5.63, p\right.$ $=.02)$ and TAU alone $\left(F_{1,188}=4.35, p=.04\right)$. Since gender could have confounded the outcomes, we corrected for this in ancillary analyses by adding gender to the model as between-group factor. This did not result in a significant outcome for the BDI-II (group $\mathrm{x}$ time: $F_{6,536}=0.94, p=.47$ ) nor was there a significant interaction between group and gender $\left(F_{2,270}=0.47, p=.63\right)$. We, therefore, omitted this correction from all further analyses.

\section{Effect sizes}

Regarding the magnitude of the effects, as can be seen in table 3, all three groups had medium to large improvement effect sizes, whereas, between-group effect sizes were trivial. Small negative between-group effects sizes were also found, indicating an effect in the opposite direction to that hypothesised.

Table 3 Improvement and between-group effect sizes based on the BDI-II in the intention-totreat population

\begin{tabular}{lllllrr}
\hline & \multicolumn{3}{l}{ Improvement effect size } & \multicolumn{3}{c}{ Between-group effect size } \\
Time point & 1. CYL & 2. TAU & 3. CYL\&TAU & $1 v 2$ & $3 v 2$ & $3 v 1$ \\
\hline 2 months & 0.71 & 0.63 & 0.57 & 0.08 & -0.06 & -0.14 \\
3 months & 0.71 & 0.69 & 0.74 & 0.02 & 0.05 & 0.03 \\
6 months & 0.86 & 0.81 & 0.89 & 0.05 & 0.08 & 0.03 \\
\hline
\end{tabular}

Note. $\mathrm{CYL}=$ Colour Your Life (CCBT offered online without support); TAU = treatment as usual; BDI-II = Beck Depression Inventory II. Improvement effect size $=\left(M_{\mathrm{t} 0}-M_{\mathrm{tk}}\right) / S D\left(M_{\mathrm{t} 0}-\right.$ $M_{\mathrm{tk}}$ ). Between group effect sizes = difference in improvement effect sizes between two groups.

\section{Reliable and clinical change}

Table 4 shows the proportion of participants in each group who showed a reliable change, a clinically significant change, and both for each time-point. There were no significant differences between the three groups (all $p>.12$ ). In figure 2 the percentage of patients with both a reliable change and a clinically significant change are graphically shown. We calculated the number needed to treat (NNT) with TAU as the reference group. At six months the NNT were 72 and 25, respectively for CYL and CYL\&TAU. 
Table 4 Proportion of patients in the intention-to-treat population showing reliable and/or clinically significant change based on the BDI-II

\begin{tabular}{llllll}
\hline Outcome & Time point & CYL & TAU & CYL \& TAU & $X^{2}(d f=2)$ \\
\hline RC & 2 months & $36(37.1 \%)$ & $30(30.9 \%)$ & $30(31.3 \%)$ & 1.06 \\
& 3 months & $35(36.8 \%)$ & $33(34.7 \%)$ & $43(45.3 \%)$ & 2.48 \\
CSC & 6 months & $45(49.5 \%)$ & $44(47.8 \%)$ & $54(58.1 \%)$ & 2.25 \\
& 2 months & $24(24.7 \%)$ & $16(16.5 \%)$ & $16(16.7 \%)$ & 2.76 \\
& 3 months & $22(23.2 \%)$ & $18(18.8 \%)$ & $28(29.5 \%)$ & 3.06 \\
RC+CSC & 6 months & $26(28.6 \%)$ & $29(31.5 \%)$ & $33(35.5 \%)$ & 1.02 \\
& 2 months & $23(23.7 \%)$ & $13(13.4 \%)$ & $14(14.6 \%)$ & 4.32 \\
& 3 months & $21(22.1 \%)$ & $16(16.7 \%)$ & $24(25.3 \%)$ & 2.15 \\
& 6 months & $26(28.6 \%)$ & $25(27.2 \%)$ & $29(31.2 \%)$ & 0.37
\end{tabular}

Note. $\mathrm{CYL}=$ Colour Your Life (CCBT offered online without support); TAU = treatment as usual; BDI-II = Beck Depression Inventory II; RC = reliable change (i.e. decrease of 9 points); CSC = clinically significant change (i.e. a score below 12 ).

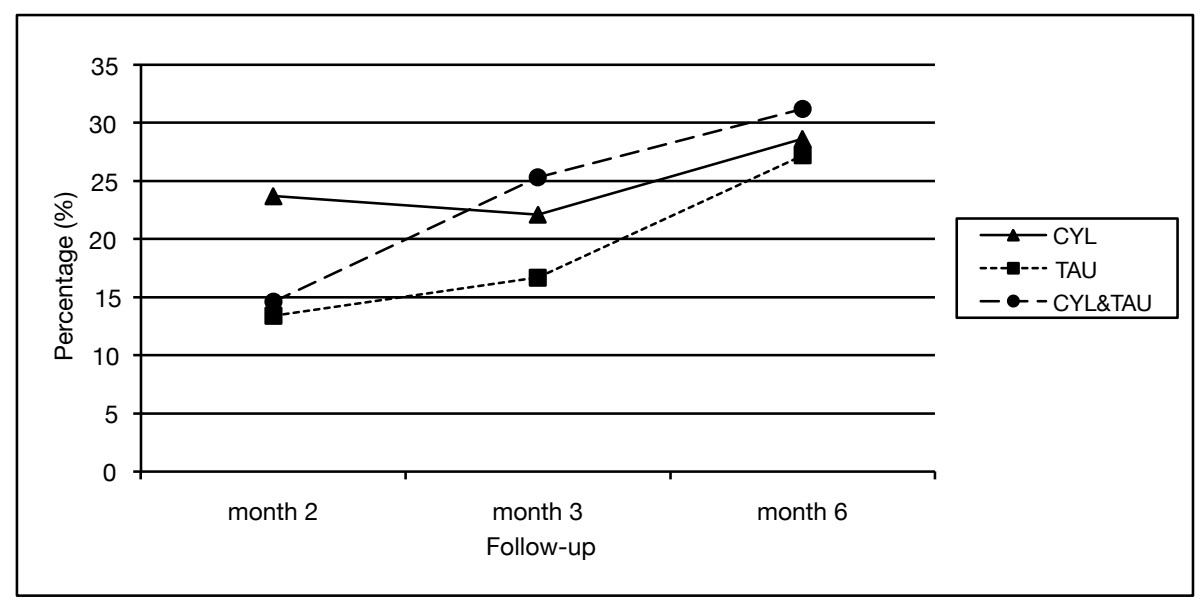

Figure 2 Percentage of patients in the intention-to-treat population with reliable and clinically significant change (RC+CSC)

Note. $\mathrm{CYL}=$ Colour Your Life (CCBT offered online without support); TAU = treatment as usual. 


\section{Treatment received}

In table 5, treatment adherence and the use of health care services are shown for each group during six months after baseline. As was expected, there were some significant differences between the three groups $(p<.05)$. More patients in TAU and CYL\&TAU visited their GP for depressive complaints compared with those in the CYL alone group. Concerning the use of CYL, more patients in the CYL\&TAU group completed the last session compared to the CYL alone group. Furthermore, more patients in the TAU group received specialist mental health care than in the other groups, and they received it earlier in time. Finally, only a small proportion of patients in each group received an adequate dosage of treatment.

Table 5 Treatment adherence and consumption of mental health care during six months of follow-up in the intention-to-treat population

\begin{tabular}{|c|c|c|c|}
\hline Variable & CYL & TAU & CYL\&TAU \\
\hline \multicolumn{4}{|l|}{ Use of GP care ${ }^{2}$} \\
\hline - Depression related GP contact, $\mathrm{nr}(\%)$ & $25(28.7 \%)$ & $66(73.3 \%)$ & $67(73.3 \%)^{\star \star}$ \\
\hline \multicolumn{4}{|l|}{ Details of those who visited the GP: } \\
\hline - Number of contacts, $M(S D)$ & $4.7(4.5)$ & $3.7(3.4)$ & $2.9(3.3)$ \\
\hline - Prescription of antidepressants, $M(S D)$ & $8(32.0 \%)$ & $25(37.9 \%)$ & $17(25.4 \%)$ \\
\hline \multicolumn{4}{|l|}{ Use of other mental health care ${ }^{a}$} \\
\hline - Use of antidepressants, $\mathrm{nr}(\%)$ & $12(13.8 \%)$ & $24(26.7 \%)$ & $23(25.0 \%)$ \\
\hline - Specialist mental health care, $\mathrm{nr}(\%)$ & $17(23.6 \%)$ & $33(36.7 \%)$ & $22(23.9 \%)^{*}$ \\
\hline \multicolumn{4}{|c|}{ Details of those who received specialist mental health care: } \\
\hline - Month of first contact, $M(S D)$ & $3.4(1.7)$ & $2.0(1.1)$ & $2.5(1.5)^{\star}$ \\
\hline Number of contacts, $M(S D)$ & $7.8(6.2)$ & $7.5(6.3)$ & $7.3(5.2)$ \\
\hline \multicolumn{4}{|l|}{ Use of the CYL program } \\
\hline - Completed first session, $\mathrm{nr}(\%)$ & $72(72.0 \%)$ & - & 76 (76.0\%) \\
\hline Completed last session, $\mathrm{nr}(\%)$ & $14(14.0 \%)$ & - & $26(26.0 \%)^{\star}$ \\
\hline _ Number of sessions, $M(S D)$ & $3.4(3.0)$ & - & $4.0(3.4)$ \\
\hline \multicolumn{4}{|l|}{ Protocol compliance } \\
\hline - Adequate dosage of TAU, $\mathrm{nr}(\%)$ & - & $31(34.4 \%)$ & $26(28.3 \%)$ \\
\hline - Adequate dosage of CYL, $\mathrm{nr}(\%)$ & $36(36.0 \%)$ & - & $47(47.0 \%)$ \\
\hline - Overall treatment compliance, $\mathrm{nr}(\%)$ & $36(36.0 \%)$ & $31(34.4 \%)$ & $12(12.9 \%)^{\star \star}$ \\
\hline \multicolumn{4}{|c|}{$\begin{array}{l}\text { Note. CYL = Colour Your Life (CCBT offered online without support); TAU = treatment as } \\
\text { usual. Adequate dosage of TAU = at least four consultations or antidepressant prescription; } \\
\text { adequate dosage of } C Y L=\text { at least five sessions. Chi-square tests for categorical variables } \\
\text { and t-tests / ANOVA's for continuous variables were used. } \\
\text { a Data unavailable: } C Y L(N=13), \text { TAU }(N=13) \text {, and CYL\&TAU }(N=8) \text {. } \\
{ }^{*} p<.05 ;{ }^{* \star} p<.001 \text {. }\end{array}$} \\
\hline
\end{tabular}




\section{Per-protocol analyses}

First, we compared the outcomes on the BDI-II only for those who adhered to the treatment. Group by time interaction was not statistically significant $\left(F_{6,148}=0.85, p\right.$ $=.53$ ). We repeated these analyses using a less strict definition of adherence in the CYL\&TAU group, i.e. adherence was defined as either an adequate dosage of CYL or an adequate dosage of TAU $(N=59)$. Again no significant interaction effect was obtained $\left(F_{6,242}=1.67, p=.13\right)$.

Next, we compared the BDI-II scores between those who adhered to the treatment protocol and those who did not within each intervention group. None of these within-group tests revealed statistically significant adherence by time interaction effects (CYL: $F_{3,87}=0.59, p=.62$; TAU: $F_{3,86}=2.03, p=.12$; CYL\&TAU: $F_{3,89}=0.76, p=$ .52). When we used the less strict definition of adherence in the CYL\&TAU group, a small effect, albeit clinically neglectable, for adherence by time interaction was obtained $\left(F_{3,88}=2.70, p=.05\right)$. Contrasts revealed no differences between those who adhered to the treatment and those who did not for each change score ( $p s>.30$ ).

\section{Sub-group analyses}

Since initial depressive severity was high (as can be concluded from the baseline BDI-II scores in table 2), we conducted ancillary subgroup analyses. First, subgroups were formed according to the initial median score on the BDI-II for the total group; low scores $=\mathrm{BDI}<27$, and high scores $=\mathrm{BDI}-\mathrm{Il} \geq 27$. No statistically significant group by time interaction effects were found in each subgroup (BDI<27: $F_{6,262}=1.27, p=$ $\left..27 ; \mathrm{BDI} \geq 27: F_{6,270}=1.15, p=.34\right)$. Second, subgroups were formed based on the presence or absence of a major depressive episode (MDE). In the no MDE subgroup, the group by time interaction was not statistically significant $\left(F_{6,84}=1.02, p=.42\right)$, while a significant interaction was obtained in the MDE group $\left(F_{6,448}=2.25, p=.04\right)$. Contrasts revealed only a significant effect for change from baseline to 3 months of follow-up in favour of the CYL\&TAU group compared to the TAU alone group $\left(F_{1,161}=\right.$ $6.03, p=.02)$. 


\section{Discussion}

\section{Main results}

In contrast to our hypotheses, the findings suggest that there are no meaningful differences between CYL, TAU, and CYL and TAU combined during six months of follow-up in terms of depressive severity, quality of life, dysfunctional beliefs, and general psychological distress. Although we found medium improvement effect sizes in depressive severity for all interventions, the between-group effect sizes were rather small or even negative. Moreover, per-protocol analysis between and within groups revealed no differences between the interventions either. Finally, we found that treatment adherence was low in all interventions. It should be noted that at three months, a significant effect was found for social functioning in favour of the combination treatment. We are reluctant to interpret this effect given the high number of statistical tests we performed.

\section{Previous studies}

To be able to compare the effects of CCBT in our study with previous ones, we calculated the usual Cohen's d (i.e. dividing the pre-post difference by the pooled standard deviation) for the 2-month follow-up period. Our improvement effect size for CYL ( $d$ $=0.84$ ) was smaller than in the previous study on CYL $(d=1.00)$ (Spek et al., 2007b) and than in the previous primary care study $(d=1.27)$ (Proudfoot et al., 2004). The between-group effect size of CYL relative to TAU was smaller $(d=0.20)$ than found for CYL in a previous study $(d=0.55)$ (Spek et al., 2007b), CCBT in primary care ( $d$ $=0.65$ ) (Proudfoot et al., 2004), online CCBT with support $(d=1.05)$ (Andersson et al., 2005), and for CCBT with shortened face-to-face therapy $(d=1.14)$ (Wright et al., 2005). Comparable effect sizes were found for bibliotherapy with minimal contact in primary care $(d=0.18)$ (Willemse et al., 2004), and online CCBT without support ( $d$ $=0.22$ ) (Spek et al., 2007a). It should be noted here that most of these studies used other comparison groups, making it difficult to directly compare the effects.

There are several reasons that may explain the small effects in our study. First of all, one might argue that the CYL itself was less effective than other CCBT programs. Results from the previous study using CYL seemed very promising for subthreshold depression in people over 50 years old (Spek et al., 2008a; Spek et al., 2007b). However, in their study, baseline assessment of the primary outcome was conducted after randomisation, which could have violated their results.

Second, the way CCBT was offered might explain the different outcomes. Various technologies can be used, ranging from telephone-system to CD-ROMs, hi-tech computers, palmtops, and Internet (Christensen et al., 2004a; Newman et al., 1999; Osgood-Hynes et al., 1998; Proudfoot et al., 2003). The latest interventions are highly sophisticated computer systems, which might stimulate and improve engagement and motivation (Cavanagh \& Shapiro, 2004). Although CYL makes full use of the current technologies, this might not have been enough to stimulate treatment adherence. We think that the lack of clinician support might account for the poor adherence and response to online, unsupported CCBT that we observed. Similar studies also showed a lack of response (Clarke et al., 2002; Patten, 2003; Spek et al., 2007a), while studies that offered some form of support reported more treatment adherence and larger effects to online CCBT for several psychological disorders (Andersson et al., 2005; Carlbring et al., 2007b; Christensen et al., 2004a; Clarke et al., 2005; Kenwright 
et al., 2005). Poor treatment adherence in our study might thus have masked potential effects of the interventions, but our ancillary per-protocol analyses did not reveal differences between the treatment-groups either. We did observe a small trend in favour of the combination group as opposed to both mono-therapies for reliable change and clinically significant change, as well as for the subgroup with a major depressive episode. However, our combination group cannot be seen as supported self-help, since the GP was not directly involved in the CCBT intervention.

Third, the low effect sizes in all three interventions might be attributable to our study sample, which was more severely depressed than in previous studies (Andersson et al., 2005; Proudfoot et al., 2004). The mean starting levels on the BDI-Il were even higher than generally seen in depressed, primary care patients (Vuorilehto et al., 2007). Chronicity might also have negatively influenced the outcome (Hamilton \& Dobson, 2002). Unfortunately, we do not have details on the exact duration of the current depressive episode. Moreover, because of our recruitment strategy our sample did not consist of active help-seekers, despite the high severity. This might have resulted in less motivated participants.

Finally, we should note that none of our interventions did particularly well. Clinical improvement was approximately $30 \%$ in all groups. Given the fact that the placebo-response rate for depression is generally high (i.e. 30-40\%) (Bialik et al., 1995; Walsh et al., 2002), we might have observed the natural course of depression in the current study, although improvement was somewhat larger as seen in patients without treatment (Posternak \& Miller, 2001).

\section{Implications}

Our findings might have several implications for the primary care treatment of depression. First, treatment might only be indicated for those who 'get stuck' in their depression, since depressive symptoms seem to improve over time without adhering to treatment, as was shown in the current study. Second, for more severely depressed patients online CCBT offered with some support might be more helpful. Third, this group of more severely depressed patients might also fare better in secondary mental health care, where they can receive psychotherapy or antidepressant medication for which larger effect sizes have been found relative to our effect size (DeRubeis et al., 2005; Dimidjian et al., 2006). Fourth, careful implementation of unsupported online self-help is warranted. Stepped-care and collaborative care models might be viable options (Katon et al., 1995; Scogin et al., 2003). Fifth, if large differences in costs between the interventions exist, this might be a reason to choose one primary care treatment over the other. Only one study so far has conducted an economic evaluation of CCBT (McCrone et al., 2004). It was shown that supported CCBT was both more effective and more costly compared with usual GP care. When willing to pay for an additional unit of effect, CCBT could be very cost-effective (McCrone et al., 2004). In a next paper, we will report the economic evaluation of CYL without support in primary care. Finally, qualitative process evaluation and information on patients' experiences (e.g. treatment satisfaction) might also help to decide which treatment should be given to a patient. The acceptability by patients of CCBT both before and after treatment (e.g. expectancy, credibility, and satisfaction) has rarely been assessed in research (Kaltenthaler et al., 2008b). Taken together, more work needs to be done to optimise treatment adherence in CCBT, to determine the best way of providing online 
and unsupported CCBT in actual practice, and to determine for whom CCBT is best suited.

\section{Methodological considerations}

We feel that our results cannot be explained by clear methodological flaws. Our large sample size $(N=303)$ provided us with sufficient power to detect significant differences between the interventions. Furthermore, we had a relatively low attrition rate, so we feel confident that no biases occurred due to missing data. Finally, we were able to recruit patients from the general population. Unlike in samples selected in general practices or clinics, no biases occurred due to help seeking behaviour of patients and illness recognition by physicians, which is often a problem in depression (Paykel et al., 1997).

Some limitations of the present study should also be noted. All our outcomes were measured online and one might question the equality of computerised questionnaires and paper-and-pen versions. However, there are sufficient indications that computerised and paper-and-pen questionnaires show similar construct validity (Butcher et al., 2004; Butcher et al., 2000; Schulenberg \& Yutrzenka, 2001). Furthermore, we relied on self-report measures at follow-up and, as a result, we have no information on actual diagnoses of depressive episodes at follow-up. Finally, it should be noted that the number of patients included is merely a fraction of the original recruitment population (i.e. $0.14 \%$ ), despite the high prevalence of depression in the community (Bijl et al., 1998; Kessler et al., 2003). Participants had to come to the research centre for an intake, which could have reduced the number of applicants, but could also have increased the compliance. Overall, the low response rate in the current study might be discouraging for the belief of many researchers that online CCBT can be disseminated to large parts of the general population (Andersson \& Cuijpers, 2008).

\section{Conclusion}

In the current study we were unable to confirm the previously reported high effectiveness of CCBT using online, unsupported Colour Your Life (CYL). Moreover, adding CYL to treatment as usual had no extra beneficial effects. These findings cannot merely be explained by the lack of treatment adherence, since per-protocol analyses showed no differences between the interventions either. It is entirely possible that we have observed natural, although not complete, recovery in a group moderately and severely depressed patients who showed a decrease in symptoms irrespective of the nature of the intervention they received. CCBT, offered online without any support, is not beneficial for all depressed patients (nor is any treatment of any kind). Adding therapist support to CYL or treatment in secondary mental health care might have yielded better results in this group of depressed patients. 


\section{Acknowledgements}

We thank Annie Hendriks and Greet Kellens for their assistance during the study and Rosanne Janssen for the development of the infrastructure for online data-collection. The trial is financed by ZonMw (Netherlands Organisation for Health Research and Development; project number 945-04-417), research institute EPP and research institute CAPHRI. Municipalities Eijsden, Meerssen, Sittard-Geleen, Valkenburg and Maastricht sponsored the study. 



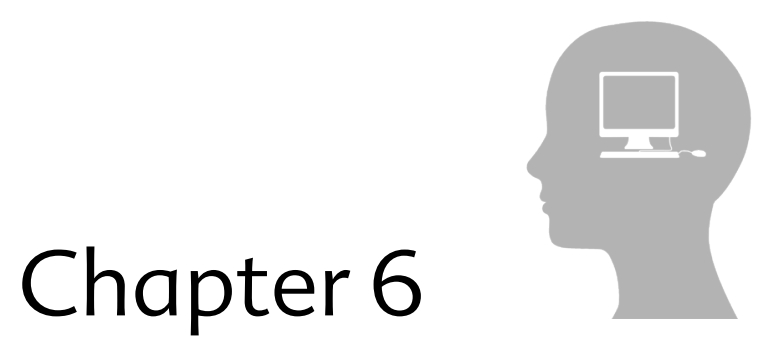

\section{One-year follow-up results of unsupported online} computerised cognitive behavioural therapy for depression in primary care: a randomised trial

L.E. de Graaf, S.A.H. Gerhards, A. Arntz, H. Riper, J.F.M. Metsemakers, S.M.A.A. Evers, J.L. Severens, G. Widdershoven \& M.J.H. Huibers 


\section{Abstract}

Background: In a recent randomised trial, we were unable to confirm the previously reported high effectiveness of computerised cognitive behavioural therapy (CCBT) for depression using an online intervention (i.e. Colour Your Life) without professional support at six months of follow-up. In this article, we present the one-year follow-up results, which have rarely been reported on CCBT. Additionally, we tested whether CCBT can prevent depressive relapse.

Methods: Depressed participants $(N=303)$ were randomly allocated to (a) online CCBT (i.e. Colour Your Life) without support (b) treatment as usual (TAU) by a GP, or (c) CCBT and TAU combined. We had a 12-month follow-up period. Primary outcome measure was the Beck Depression Inventory II. Self-reported health care use was also measured.

Results: At 12 months, no statistically significant differences between the three interventions were found in the intention-to-treat population for depressive severity, reliable improvement, remission, and relapse. A small trend was observed for reliable improvement in favour of CCBT\&TAU. In the first quarter, differences in health care consumption are significant (i.e. less GP contacts, antidepressant medication, and specialist mental health care in CCBT), but they disappear over time.

Conclusion: Unsupported online CCBT was not superior to TAU by a GP for depression. Combination treatment might have additional effects on the longer-term. With equal effects, CCBT led to reduced health care consumption. Overall effects were modest in all treatments, which might be explained by the undertreatment we observed in all groups. 


\section{Introduction}

Although cognitive behavioural therapy (CBT) is an effective treatment for depression (Butler et al., 2006; Hollon et al., 2006), many depressed patients in primary care remain untreated (Kessler et al., 2003). A possible solution might be computerised CBT (CCBT), in which therapy components are delegated to a computer system. CCBT programmes can vary greatly in terms of technologies used and amount of additional professional support. CCBT seems a feasible, effective, and acceptable treatment for depression (Kaltenthaler et al., 2006; Kaltenthaler et al., 2008a; Spek et al., 2007a). However, only one study so far investigated the efficacy of CCBT for depression in primary care (Proudfoot et al., 2004). It was shown that CCBT (delivered on a personal computer located in the general practice with nurse support) is more effective than usual GP care in mild to moderate depression. We recently evaluated the short-term effectiveness of unsupported online CCBT (i.e. Colour Your Life) for depression in primary care compared with treatment as usual by a GP (TAU) and a combination of both treatments (de Graaf et al., in press-a). In contrast to the Proudfoot study (2004), we found no differences in outcome between CCBT, TAU, and the combination of CCBT and TAU during six months of follow-up. To date, long-term follow-up results of CCBT for depression have rarely been reported. We are aware of only two studies that have reported one-year follow-up results and they showed promising results for CCBT (see Mackinnon et al., 2008; Spek et al., 2008a). To contribute to these findings, we present the effects of the three interventions after twelve months. Despite the results found during the first six months, we additionally tested whether unsupported online CCBT can prevent depressive relapse. Relapse is very common in depression (Judd, 1997), and failing to achieve adequate maintenance treatment has serious personal and economic consequences (Keller \& Boland, 1998). Previous studies have demonstrated that face-to-face CBT prevents future relapse and recurrence (Hollon et al., 2006; Vittengl et al., 2007), but no such studies have tested this in computer-assisted treatments. Since the level of health care consumption during the follow-up period might influence outcome and relapse rates, we also examined the use of antidepressant medication and treatment in specialist mental health care. 


\section{Method}

\section{Design}

The design of the study was a randomised trial with three conditions: (a) unsupported online CCBT, (b) TAU by a GP, and (c) CCBT and TAU combined. Details of the study method have been described elsewhere (de Graaf et al., 2008a).

\section{Study population}

Participants were recruited in the general population by means of a large-scale Internet-based screening in the South of the Netherlands. A random selection of individuals $(N=217,816)$ was sent a postal invitation to participate in the screening. Potentially eligible participants were invited to visit the research centre for an intake to assess final eligibility. Inclusion criteria were: age 18 to 65 ; access to the Internet at home; at least mild to moderate depressive complaints (BDI-II score $\geq 16$ ); duration of depressive complaints 3 months or more; no current psychological treatment for depression; no continuous antidepressant treatment for at least 3 months prior to entry; fluent in Dutch language; no alcohol and/or drug dependence; and no severe psychiatric co-morbidity.

\section{Procedure}

After written informed consent was obtained, participants were randomly allocated to one of the three interventions. Those in the CCBT and CCBT\&TAU condition were given secure login codes for Colour Your Life (CYL), which is an online, multimedia, interactive, self-help computer program for overcoming subthreshold depression (Riper \& Kramer, 2004). No professional assistance was offered. Participants in the TAU and CCBT\&TAU condition were advised to contact their own GP. The GPs were advised to follow depression guidelines described by the Dutch College of General Practitioners (van Marwijk et al., 2003).

Baseline assessment took place on a computer at the research centre before randomisation. All follow-up assessments took place at home via the Internet at two, three, six, nine, and twelve months after inclusion. Preceding an upcoming assessment point, participants received an e-mail alert.

\section{Outcome}

The primary outcome was the severity of depression as measured with the Beck Depression Inventory Second Edition (BDI-II), high scores indicating severe depression (range 0-63). There has been consistent support for its construct validity and reliability in various samples (Arnau et al., 2001; Beck et al., 1996; van der Does, 2002). Additionally, a health care use questionnaire was used monthly to measure self-reported use of GP care, antidepressant medication, and specialist mental health care.

\section{Data analyses}

Preliminary tests for distribution and outliers did not indicate substantial deviations from normality for the BDI-II. Only intermittent missing data were imputed $(N=5)$ by calculating the mean of the values of a previous and a subsequent time point. Missing data due to loss to follow-up were not replaced by imputed values. We tested all effects at the $p<.05$ level. All analyses were carried out using SPSS (version 15.0.1 for Windows). 
All analyses were based on the intention-to-treat principle (i.e. those who provided follow-up data irrespective of treatment adherence). First, repeated-measures analyses of variance (ANOVAs) were performed for the BDI-II. Second, improvement effect sizes for BDI-II scores at 12 months of follow-up were calculated, defined as $\left(M_{t 0}-M_{t k}\right) / S D\left(M_{t 0}-M_{t k}\right)$. We did not use the pooled $S D$ as proposed by Cohen (1988), since this can lead to under- or overestimation of the effect size. Between group effect sizes were determined by calculating the difference in improvement effect sizes between two groups. Third, we determined the proportion of patients who made clinically meaningful changes at 12 months using the methodology of Jacobson and Truax (1991): (1) reliable improvement, based on reliable change index (i.e. a decrease of at least 9 points on the BDI-II since baseline), and (2) remission, defined as clinically significant change (i.e. a score below 12 on the BDI-II). Fourth, we calculated the relapse rate in those patients who were reliably improved at 3 months of follow-up, immediately after the CCBT intervention. Relapse was defined as an increase of at least 9 points on the BDI-II from 3 months to either 6 , 9, or 12 months of follow-up. Chisquare tests were used to examine frequency differences between the three groups. Finally, to better understand the obtained findings, we examined quarterly health care consumption. This was defined as making at least one visit for depression treatment to either a GP or a specialist in mental health care per quarter, or using antidepressant medication in that quarter. To compare frequency differences across the groups Chisquare tests were used. 


\section{Results}

Three-hundred-and-three participants were enrolled in the study. At 12 months of follow-up, data were available for 267 participants (attrition rate 11.9\%). The flow of the participants is graphically shown in figure 1. Demographic characteristics have been described in detail elsewhere (see de Graaf et al., in press-a). To summarise, male gender was distributed as follows: CCBT (48\%), TAU (45\%), and CCBT\&TAU (37\%). Despite randomisation, gender was not equally distributed. In a previous paper (de Graaf et al., in press-a), we showed that gender did not confound the outcomes. Mean age for CCBT, TAU, and CCBT\&TAU was 44.3 years $(S D=11.8), 45.1$ years $(S D$ $=12.2)$, and 45.2 years $(S D=10.9)$ respectively.

Mean BDI-II scores are shown in table 1. Repeated measures ANOVA showed that there was no significant group by time interaction on the BDI-II.

At 12 months, improvement effect sizes in the three groups were all exactly the same (i.e. 1.0 for each group). Between-group effect sizes were, therefore, zero.

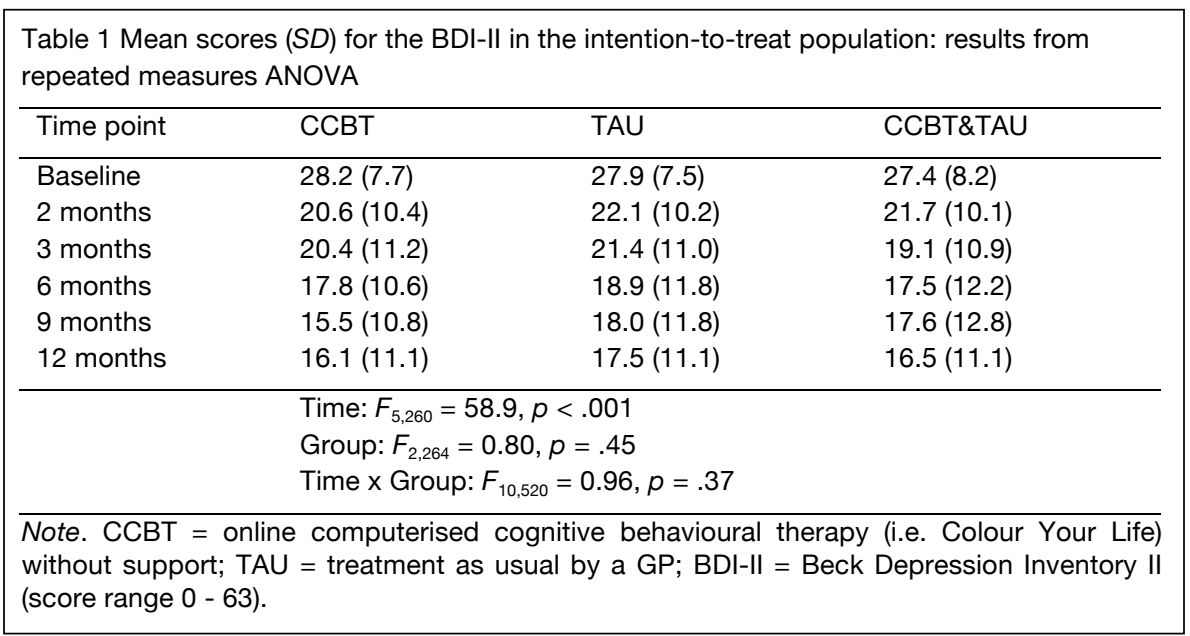




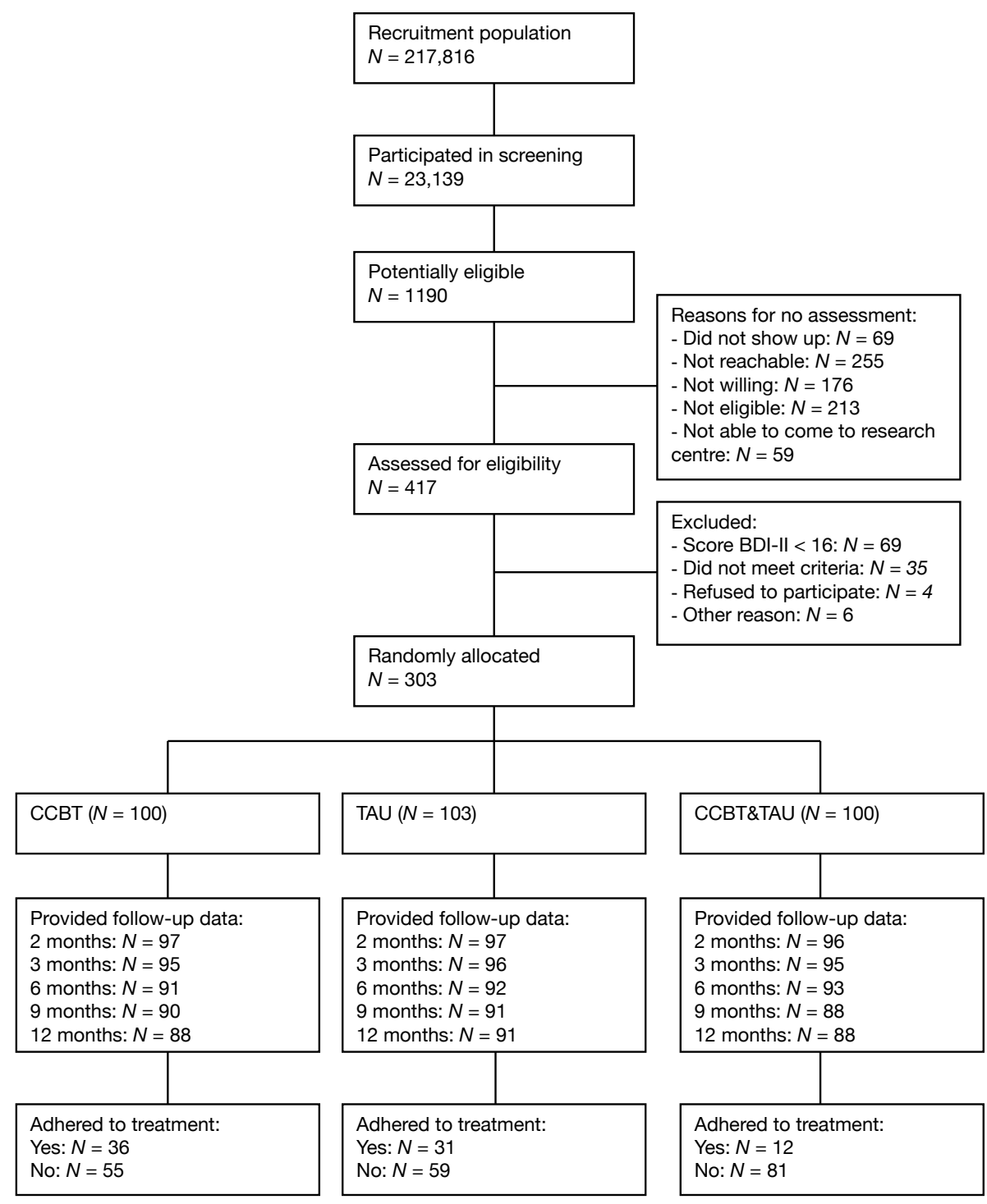

\section{Figure 1 Flowchart}

Note. $\mathrm{CCBT}=$ online computerised cognitive behavioural therapy (i.e. Colour Your Life) without support; TAU = treatment as usual by a GP. 
The number of participants who showed a reliable improvement (i.e. a decrease of at least 9 points on the BDI-II from baseline) at 12 months was 47 (53.4\%) for CCBT, 46 (50.5\%) for TAU and 54 (61.4\%) for CCBT\&TAU (see figure 2). Although the proportions did not differ significantly between the groups $\left(X^{2}(2)=2.26, p=.32\right)$, there seems to be a small trend in favour of CCBT\&TAU. Moreover, figure 2 seems to show a cyclic-like pattern; reliable improvement in CCBT\&TAU declines at 9 months and rises again at 12 months, while it decreases at 12 months in the other two groups.

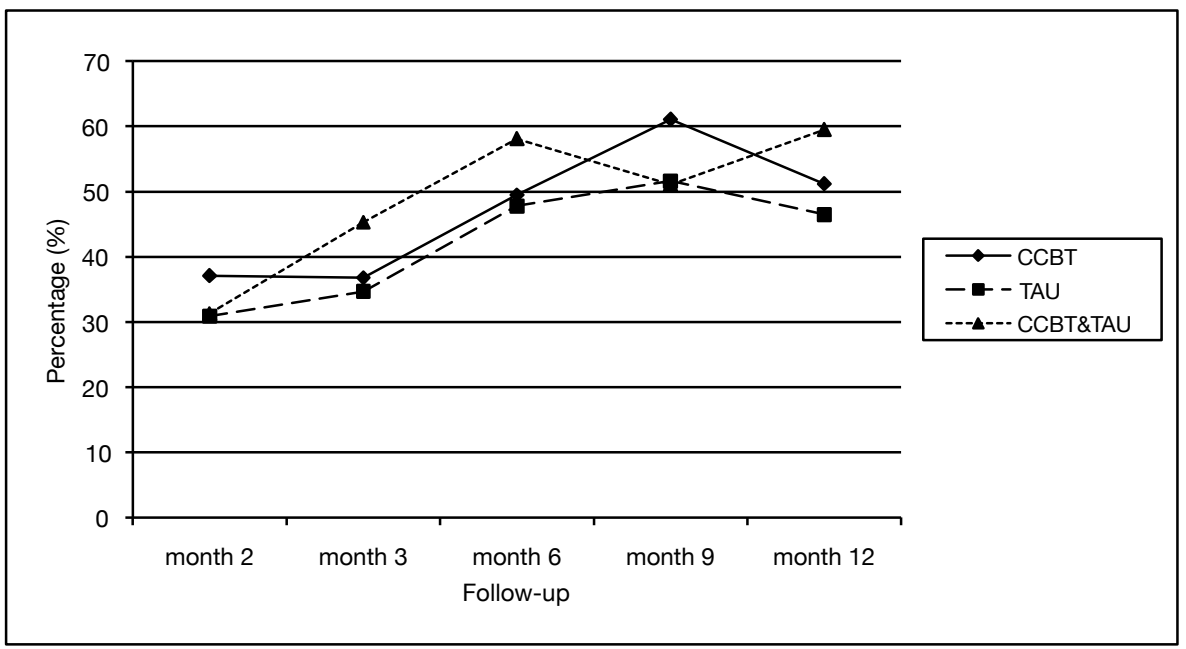

Figure 2 Percentage of patients with reliable improvement (i.e. a reduction of at least 9 points on the Beck Depression Inventory II since baseline)

Note. $\mathrm{CCBT}=$ online computerised cognitive behavioural therapy (i.e. Colour Your Life) without support; TAU $=$ treatment as usual by a GP.

There was no significant difference in the number of participants who had remitted (i.e. a score below 12 on the BDI-II) at 12 months $\left(X^{2}(2)=0.30, p=.86\right)$ : $36(40.9 \%)$ for CCBT, 34 (37.4\%) for TAU, and 33 (37.5\%) for CCBT\&TAU.

Relapse rates in those who were reliably improved at 3 months (i.e. CCBT: $N=$ 35; TAU: $N=33$; CCBT\&TAU: $N=43)$ seemed lower in the TAU group $(20.7 \% ; N=6)$ than in the CCBT group (31.3\%; $N=10)$ and the CCBT\&TAU group (31.0\%; $N=13)$. However, these differences were not statistically significant $\left(\mathrm{X}^{2}(2)=1.11, p=.57\right)$.

Table 2 shows the quarterly consumption of mental health care. Only in the first quarter, more patients in TAU and CCBT\&TAU had depression related GP contact than those in the CCBT alone group. In the first quarter, the TAU group received specialist mental health care more frequently than the other two groups. In the TAU and CCBT\&TAU groups, use of antidepressants was more frequent than in the CCBT alone group (albeit borderline statistically significant). On average, it was observed that antidepressant use remained fairly stable over time, while GP contact and specialist mental health care decreased. Regarding the use of CCBT, most patients started the intervention, but many did not finish all modules. 
Table 2 Quarterly consumption of mental health care and use of CCBT.

Data are in numbers (\%).

\begin{tabular}{|c|c|c|c|c|c|}
\hline Variable & CCBT & TAU & CCBT\&TAU & $X^{2}(d f)$ & $p$ \\
\hline \multicolumn{6}{|c|}{ Depression related GP contact } \\
\hline - $1^{\text {st }}$ quarter & $19(20.7 \%)$ & $66(70.2 \%)$ & $63(67.0 \%)$ & $57.2(2)$ & $<.001$ \\
\hline - $2^{\text {nd }}$ quarter & $18(20.0 \%)$ & $23(25.3 \%)$ & $22(23.9 \%)$ & $0.7(2)$ & .68 \\
\hline$-3^{\text {rd }}$ quarter & $11(12.2 \%)$ & $15(16.5 \%)$ & $12(13.6 \%)$ & $0.7(2)$ & .70 \\
\hline - $4^{\text {th }}$ quarter & $12(13.6 \%)$ & $15(16.5 \%)$ & $13(14.8 \%)$ & $0.3(2)$ & .87 \\
\hline \multicolumn{6}{|c|}{ Use of antidepressants } \\
\hline - $1^{\text {st }}$ quarter & $8(8.7 \%)$ & $20(21.3 \%)$ & $17(18.1 \%)$ & $5.9(2)$ & .05 \\
\hline$-2^{\text {nd }}$ quarter & $10(11.1 \%)$ & $22(24.2 \%)$ & $19(20.7 \%)$ & $5.4(2)$ & .07 \\
\hline - $\quad 3^{\text {rd }}$ quarter & $11(12.2 \%)$ & $20(22.0 \%)$ & $19(21.6 \%)$ & $3.6(2)$ & .16 \\
\hline - $\quad 4^{\text {th }}$ quarter & $8(9.1 \%)$ & $19(20.9 \%)$ & $19(21.6 \%)$ & $6.1(2)$ & .05 \\
\hline \multicolumn{6}{|c|}{ Specialist mental health care } \\
\hline$-1^{\text {st }}$ quarter & $11(12.0 \%)$ & 29 (30.9\%) & $19(20.2 \%)$ & $10.0(2)$ & .01 \\
\hline - $2^{\text {nd }}$ quarter & $14(15.6 \%)$ & $22(24.2 \%)$ & $13(14.1 \%)$ & $3.7(2)$ & .16 \\
\hline$-3^{\text {rd }}$ quarter & $15(16.7 \%)$ & $21(23.1 \%)$ & $17(19.3 \%)$ & $1.2(2)$ & .55 \\
\hline - $4^{\text {th }}$ quarter & $12(13.6 \%)$ & $15(16.5 \%)$ & $8(9.1 \%)$ & $2.2(2)$ & .34 \\
\hline \multicolumn{6}{|l|}{ Use of CCBT } \\
\hline $\begin{array}{l}\text { - First session } \\
\text { completed }\end{array}$ & $72(72.0 \%)$ & - & $76(76.0 \%)$ & $0.4(1)$ & .52 \\
\hline $\begin{array}{l}\text { - Last session } \\
\text { completed }\end{array}$ & $14(14.0 \%)$ & - & $26(26.0 \%)$ & $4.5(1)$ & .03 \\
\hline
\end{tabular}




\section{Discussion}

The one-year follow-up results of the effectiveness of unsupported online CCBT (i.e. Colour Your Life) for depression look fairly similar to the results for six months of follow-up reported in our previous paper (de Graaf et al., in press-a). Reductions in depressive severity were sustained and remained comparable over time between the three interventions (i.e. CCBT, TAU, and CCBT\&TAU).

The comparison of our findings with the few available studies that reported longterm outcomes of CCBT for depression falls short. At one-year of follow-up, CCBT (i.e. Colour Your Life) for people over 50-years old was more effective than a waiting list (Spek et al., 2008a), as was online CCBT with support relative to an attention control group (Mackinnon et al., 2008). We compared unsupported CCBT with an active treatment group, but found that CCBT did not outperform usual GP care and the combination of both did not have additional effects. As shown in our previous paper (de Graaf et al., in press-a), this might not merely result from the poor treatment adherence in our study. It might also be attributable to the absence of therapist' support in CCBT in our study (e.g. see Spek et al., 2007a) and to our study population which was more severely depressed than in previous CCBT studies (e.g. Andersson et al., 2004; Proudfoot et al., 2004; Spek et al., 2007b). However, we found that with equal effects even a small dosage of CCBT was associated with less use of antidepressant medication during twelve months, and with less visits to the GP and specialist mental health care during the first quarter.

The finding that depressive severity remained unchanged subsequent to six months in the three groups can be interpreted in two distinct ways. One might argue that depression status was maintained and did at least not deteriorate over time. Alternatively, one might say that no additional improvements were made and patients were still fairly depressed after twelve months of follow-up. Despite the finding that the majority of patients made reliable improvements, the overall effects of our interventions were rather modest regarding effect sizes, depressive severity scores at twelve months, and remission. This might be attributed to the high amount of undertreatment in our study. As expected, health care consumption differs between groups in the first quarter, but they disappear over time. The use of health care services decreased in all groups, despite the lack of substantial improvements. This seems to suggest that patients do not step up to a more intense treatment when initial treatment proves inadequate. We can only speculate about the causes of undertreatment in the current study. On the one hand, patients themselves might have failed to seek further treatment. Patients might lack awareness and knowledge of the disorder, they might have accepted their lesser state of well-being, or they might have refused further treatment. On the other hand, GPs and professionals in specialist mental health care might not have offered alternative treatments when improvement stayed behind (see also Brugha \& Bebbington, 1992; Kessler et al., 2003), or maybe they were not able to motivate their patients. Taken together, these findings confirm that many depressed patients in primary care remain untreated (Kessler et al., 2003). It highlights the importance of systematic monitoring of depressive patients to improve the quality of care.

Initial depressive severity in our sample was high; even higher than generally seen in depressed primary care patients (Vuorilehto et al., 2007). The gap between this high depressive severity and actual treatment received is striking, since we know 
from previous research that incomplete recovery is a strong predictor of relapse and chronicity (Judd et al., 2000). In case of ongoing symptoms, further treatment is recommended. Since it has been shown that depressive symptoms are weakly associated with treatment seeking behaviour by patients, public awareness and knowledge of depression should be increased (Galbaud du Fort et al., 1999). From a steppedcare perspective, frequent monitoring should be encouraged in order to timely identify those who require stepping up to a higher level of care to ensure full recovery (Bower \& Gilbody, 2005).

A small trend (albeit statistically insignificant) was observed in favour of the combination group as opposed to both mono-therapies for reliable improvement. Given the high depressive severity of our sample, this might not seem surprising. For more severely depressed patients, combination treatments have been found to be superior to single treatments (see Friedman et al., 2004; Pampallona et al., 2004). It should be noted that no trend in favour of combination treatment was observed regarding remission. Perhaps in this group of patients, low-intensity combination treatment can reduce depressive severity, but its effect is still not enough for full remission.

Relapse rates did not significantly differ between the groups. Although the small numbers warrant some caution, a lower relapse rate was found in the TAU only group, indicating a relapse prevention trend in favour of this group. This seems in contrast to the findings from several studies that have shown that face-to-face CBT is better in preventing relapse than pharmacotherapy (Hollon et al., 2006; Paykel et al., 2005; Vittengl et al., 2007). However, more patients in the TAU group turned to specialist mental health care than those in the other groups. It is likely that they might have received face-to-face psychotherapy, such as CBT. Given the fact that we observed a trend while only $30 \%$ of TAU patients received specialist mental health care in the first quarter, this could suggest that our study population might have fared better in secondary mental health care, where personal contact might have increased illness insight and motivation for treatment. We should note here that defining relapse in improved patients at three months was an arbitrary choice. At three months, not all patients might have finished their treatments yet.

It is notable that at nine months there is a decline in the percentage of reliable improvement in the combination group, which rises again at twelve months. In contrast, in both mono-therapies the percentage of reliable improvement at twelve months decreases. We can only speculate about the meaning of this cyclic-like pattern, as it is difficult to disentangle the relationship between improvement, treatment type, and consumption of additional health care.

We feel that our results cannot be explained by clear methodological flaws. Our sample size was large and we had a relatively low attrition rate, so we feel confident that no biases occurred due to missing data. Some methodological limitations should also be acknowledged. One might question the equality of computerised questionnaires and paper-and-pen versions. However, there are sufficient indications that they show similar psychometric properties (Carlbring et al., 2007a; Garb, 2007; Schulenberg \& Yutrzenka, 2001). Furthermore, we have no information on actual diagnoses of depressive episodes at follow-up, since we relied on self-report measures. Moreover, records from GPs and specialist in mental health care might have increased the quality of the data on health care consumption.

In conclusion, improvements in depressive severity were maintained during 
twelve months of follow-up for all groups. Although unsupported online CCBT (i.e. Colour Your Life) was not more effective than TAU or CCBT\&TAU, it led to reduced health care consumption. CCBT\&TAU might have additional effects in the longer-term, but it did not have a larger relapse prevention effect than both mono-treatments. The overall effects in all groups were modest, which might be explained by the high illness severity in our sample and the undertreatment we observed. CCBT with therapist support or more intense treatment in secondary mental health might have yielded better results in this group of more severely depressed patients. The latter is contrasted by our finding that patients (and GPs and other specialists) seldomly stepped up to more intense treatment when improvement stayed behind, which might reflect barriers to seek or provide additional care. This calls for a need for systematic monitoring of patients to encourage further treatment. 


\section{Acknowledgements}

The trial is financed by ZonMw (Netherlands Organisation for Health Research and Development; project number 945-04-417), research institute EPP, and research institute CAPHRI. Municipalities Eijsden, Meerssen, Sittard-Geleen, Valkenburg, and Maastricht sponsored the study. We thank Annie Hendriks and Greet Kellens for their assistance during the study and Rosanne Janssen for the development of the infrastructure for online data-collection. 



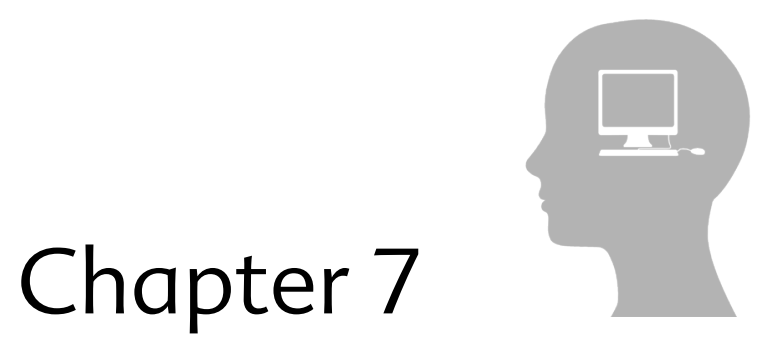

\section{Economic evaluation of online} computerised cognitive behavioural therapy without support for depression in primary care: a randomised trial

S.A.H. Gerhards, L.E. de Graaf, L.E. Jacobs, J.L. Severens, M.J.H. Huibers, A. Arntz, H. Riper, G. Widdershoven, J.F.M. Metsemakers \& S.M.A.A. Evers 


\section{Abstract}

Background: Recently, we compared the clinical effectiveness of unsupported, online computerised cognitive behavioural therapy (CCBT) with treatment as usual (TAU), and a combination of CCBT and TAU (CCBT\&TAU) for depression. Evidence on the cost-effectiveness and cost-utility of CCBT is still limited.

Aims: To assess the cost-effectiveness of CCBT compared with TAU and CCBT\&TAU.

Method: Costs, depression severity and quality of life were measured during 12 months. Cost-effectiveness and cost-utility analyses were performed from a societal perspective. Uncertainty was dealt with by bootstrap replications and sensitivity analyses.

Results: Costs were lowest for the CCBT group. There are no significant group differences in effectiveness or quality of life. Cost-utility and cost-effectiveness analyses tend to be in favour of CCBT.

Conclusion: CCBT seems the most efficient treatment strategy, although all treatments showed low adherence rates and modest improvements in depression and quality of life. 


\section{Background}

Major depression is a common mental health problem in the general population (Bijl et al., 1998) and is associated with substantial decreases in quality of life (Bijl \& Ravelli, 2000; Kruijshaar et al., 2003). Cost-of-illness studies reveal that the economic burden of depression is considerable(Cuijpers et al., 2007a; Luppa et al., 2007; Slobbe et al., 2006; Sobocki et al., 2006; Stewart et al., 2003). However, many depressed patients in primary care do not receive the care they need. Pharmacotherapy is only effective in severely depressed patients, while psychological treatments in primary care are scarce and costly. Consequently, effective treatments like cognitive behavioural therapy (CBT) (Butler et al., 2006; Hollon et al., 2006) are not offered to all patients and many depressed patients in primary care remain untreated (Hirschfeld et al., 1997). An effective, acceptable, and feasible solution for depressed patients in primary care might be computerised CBT (CCBT) (Kaltenthaler et al., 2006). In the UK, the National Institute of Health and Clinical Excellence recommended the use of two CCBT software programs for depression and anxiety (NICE, 2006), and these programs are now actually in use in the UK. In the Netherlands, an online CCBT program is currently being used as part of an ongoing implementation study (https://www.kleurjeleven.nl). However, evidence on the cost-effectiveness of CCBT is still limited (Kaltenthaler et al., 2006). To our knowledge, only one study so far conducted an economic evaluation of CCBT for depression, showing that CCBT (delivered on a personal computer in the general practice) can be highly cost-effective compared with usual care by a GP in the UK setting (McCrone et al., 2004). The cost-effectiveness of unsupported, online CCBT remains to be evaluated. In a recent study, we compared the clinical effectiveness of unsupported, online CCBT (i.e. Colour Your Life) with treatment as usual (TAU) by a GP, and a combination of both CCBT and TAU for depression (CCBT\&TAU) (de Graaf et al., in press-a). In the present study, we assess the cost-effectiveness of these three interventions, focusing on the research question: Is CCBT compared with TAU or CCBT\&TAU for mild to moderate depression preferable in terms of costeffectiveness from a societal perspective? 


\section{Method}

\section{Design and participants}

In a randomised trial, 303 participants were recruited from the general population by means of a large-scale Internet-based screening in the South of the Netherlands. Inclusion criteria were: age 18 to 65; access to the Internet at home (no dial-up connection); at least mild to moderate depressive complaints (BDI-II score $\geq 16$ ); duration of depressive complaints 3 months or more; no current psychological treatment for depression; no continuous antidepressant treatment for at least 3 months prior to entry; fluent in Dutch language; no alcohol and/or drug dependence; and no severe psychiatric co-morbidity (e.g. psychotic disorders). During a one-year follow-up period, participants were asked to fill in monthly Internet questionnaires (de Graaf et al., in press-a; de Graaf et al., 2008a).

\section{Interventions}

CCBT is based on the principles of cognitive behavioural therapy. The CCBT program in our trial (named "Colour Your Life" (CYL, in Dutch Kleur je Leven)) is an online, multimedia, interactive computer program for depression. The program consists of eight weekly sessions and a ninth booster session. At the end of each session homeworkassignments are given, such as keeping a 'mood diary'. No professional assistance was offered. Patients could reach the CCBT program wherever they had a computer with Internet access.

The GP is the major health care provider involved in the primary TAU of depression. In the Dutch health care system the GP is seen as a gatekeeper, and a key figure in the detection and treatment of depression (CBO, 2005). Participants who received the TAU were advised to contact their own GP. After inclusion, the patients' GP received an information letter about the patient's participation in the study. In the letter, the GP was advised to follow the depression guideline as described by the Dutch College of General Practitioners. Usual care as such consists of four to five biweekly consultations in combination with antidepressant treatment if indicated (Nederlands Huisartsen Genootschap [Dutch College of General Practitioners], 2003).

Further details of the design of the trial, the interventions and the population can be found elsewhere (de Graaf et al., in press-a; de Graaf et al., 2008a).

\section{Cost measures and valuation}

The economic evaluation was performed from the societal perspective. Costs were divided into the following categories: health care sector costs, costs for the patient and family, and productivity costs (Drummond et al., 2005). Due to the impact depression can have on a patient's overall health status, functioning and well-being, it is often not clear whether the health care use or productivity loss is depression-related. We therefore included all related and unrelated costs in the analysis.

Health care costs were measured by means of a monthly health care use questionnaire. Patients were asked to report the volumes of psychological, paramedical, medical, paid and informal care, participation in a self-help group, and alternative treatments received during the last month. The use of CCBT was based on computerregistered login data of the CYL program.

The patient and family costs consisted of costs of travelling and lost time due to the intervention TAU and/or CCBT. The time spent by a patient on CCBT was tracked 
by means of the computer-registered login and logout data of the program. In the health care use questionnaire, the average duration of a GP consult was registered by the patient. For the time spent on psychological care received from a psychiatrist, we used mean time duration of 30 minutes per session. The time spent on a psychologist, social-psychiatric nurse or other mental health care professional was valued by 45 minutes per session. We assumed that patients used the TAU and CCBT program outside working hours, and therefore valued their time costs as leisure time. The number of GP consults informs on the number of travels from/to the GP, and was linked to the average travel distances to a GP in the Netherlands (Oostenbrink et al., 2004; Oostenbrink et al., 2002).

Modules of the PROductivity and DISease Questionnaire (PRODISQ) were used to measure absenteeism, presenteeism and compensation mechanisms of absenteeism (Koopmanschap et al., 2004; Koopmanschap, 2005). Productivity costs were based on the outcomes of the PRODISQ, and calculated according to the friction cost method (Koopmanschap et al., 1995; Oostenbrink et al., 2004; Oostenbrink et al., 2002).

Costs for computer and Internet use were considered to be sunk costs and not included, as the inclusion criteria of the trial required that the participant has Internet access at home. For the costs of CYL we included $€ 50$ per user for the whole program and the entire intervention period (Heleen Riper, personal communication, December 8, 2008).

Dutch standard costs were used to value the health care, patient and family, and productivity cost items (Oostenbrink et al., 2004; Oostenbrink et al., 2002). If for specific categories standard costs were unavailable, we used average tariffs. Medication costs were based on the Dutch Pharmacotherapeutic Compass (College voor Zorgverzekeringen, 2007). The standard costs and tariffs of health care practitioners were the integral costs, being all costs directly and indirectly attributable to the cost unit.

Costs are presented Euros for the year 2007. Since the follow-up period lasts one year and no extrapolation over time was executed, discounting of costs was not necessary. If needed, costs were indexed to the year 2007 by means of the consumer price indexes of the Dutch Central Bureau of Statistics.

\section{Outcome measures}

Depression severity was measured with the Beck Depression Inventory Second Edition (BDI-II). The total score is the sum of the 21 items with a range of 0 (no depression) to 63 (severe depression). There has been consistent support for the construct validity and reliability of the BDI-II in various samples (Arnau et al., 2001; Beck et al., 1996; van der Does, 2002).

Quality of life was measured with the EuroQol EQ-5D and the Short Form 6D (SF-6D). The EQ-5D consists of five health state dimensions (mobility, self-care, usual activity, pain/discomfort and anxiety/depression) on which the respondent has to indicate his own health state (Brooks, 1996; EuroQol Group, 1990). An advantage of the EuroQol is that it is short and that an overall utility score for population-based quality of life can be obtained, which facilitates comparisons with other interventions and health states in other disease areas. A utility refers to the preference that individuals or society may have for any particular set of health outcomes. It is indicated by a number between 0 (the worst imaginable condition: death) and 1 (perfect health) (Drummond 
et al., 2005). Standardised value sets are available to calculate the utility based on the EQ-5D. This study used the UK tariff and the Dutch tariff to value generic quality of life (Dolan, 1997; Lamers et al., 2006; Szende et al., 2007).

The SF-6D is a utility instrument based on the health-related quality of life questionnaire 36-item short-form Health Survey (SF-36). The utility score is derived from 11 items of the SF-36 and is composed of six dimensions of health (physical functioning, role limitations, social functioning, pain, mental health, and vitality). The SF-6D utilities were derived by means of the preference-based UK tariff (Brazier et al., 2002; Brazier \& Roberts, 2004).

The utility scores of the EQ-5D and the SF-6D were used to calculate the quality adjusted life year (QALY) during the follow-up period by adjusting the length of time affected through the health outcome by the utility value (Drummond et al., 2005).

\section{Analyses}

All available data were used. Only intermittent missing data were replaced by mean imputation using the outcomes on the previous and next measurement moment of the patient. Only 8 participants had intermittent missing data. In all these cases, the number of missing follow-up moments was limited to one moment. Data of patients lost to follow-up were not imputed.

For each patient, volumes of care, travels, lost time for receiving care and lost productivity hours were multiplied by the prices determined for each cost item. Based on the costs per item, costs during the follow-up period were calculated as the cumulative costs per patient at 12 months after baseline. The costs during the follow-up period of the three groups were compared by the non-parametric bootstrapping method with $95 \%$ confidence intervals in percentiles. By bootstrapping, samples of the same size as the original data are drawn with replacement from the observed data (Briggs et al., 1997). The quality of life and severity of depression outcomes during the follow-up period were compared between the three groups using ANOVA, Kruskall-Wallis and $\mathrm{X}^{2}$ tests at the $p<.05$ level. Baseline corrections for societal cost and QALY outcomes were performed by means of a regression correction (Brunenberg et al., 2005; Manca et al., 2005; van Asselt et al., 2009).

The analysis consisted of a base-case cost-effectiveness and cost-utility analysis, and sensitivity analyses. In the base-cases, the primary outcome measure for the cost-effectiveness analysis was change from baseline score of depression severity measured by the BDI-II at 12 months, and for the cost-utility analysis the QALY at 12 months based on the EQ-5D using the UK-tariff. Costs in the base-case analysis are calculated according to the societal perspective.

Uncertainty of parameter estimates of the base-cases was dealt with by the sensitivity analyses (Drummond et al., 2005). In the sensitivity analyses, the correction method for baseline differences in costs and utilities was varied by using no correction (Manca et al., 2005; van Asselt et al., 2009). Other aspects of sensitivity analyses were: varying the time horizon of the cost-effectiveness analysis from 12 months to 6 months, varying the societal costs to the healthcare costs, calculating productivity costs according to the human capital approach instead of the friction cost method, using the Dutch tariff to value the EQ-5D, varying the QALY outcome by using the SF$6 \mathrm{D}$, and using the reliable change index of the BDI-II score as outcome measure. For the reliable change we used the methodology of Jacobson and Truax (1991), which 
gives the proportion of patients who made clinically meaningful changes at 12 months using the BDI-II (i.e. a decrease of at least 9 points on the BDI-II since baseline). Due to uncertainty about the cost price of self-help online CCBT in real-world, this cost price was varied by estimating either one fixed price per package user ( $€ 5, € 50$, and $€ 150$ ), and a price per lesson. Based on the price estimates per package, and an average number of 5 lessons completed by CCBT users, we varied this cost price by $€ 1$, $€ 10$ and $€ 30$ per lesson.

Because of the comparison of three strategies in this trial, an incremental approach is not feasible for analysing the cost-effectiveness data. Therefore the net benefit (NB) was used to present the cost-utility results in monetary units for each strategy. The NB is calculated by valuing the utility outcome in monetary values using the threshold willingness-to-pay for a QALY, minus the costs of the interventions (Stinnett \& Mullahy, 1998).

Non-parametric bootstrap re-sampling techniques were used to explore uncertainty around estimates of cost-effectiveness and cost-utility derived from the study sample (Briggs et al., 1997). For each bootstrap iteration, using a range of cost-effectiveness thresholds it was decided which strategy had the highest NB and for all iterations, and it was calculated which strategy had the highest probability of being most efficient. The resulting decision uncertainty is represented graphically by means of a cost-effectiveness acceptability curve (CEAC) (Briggs, 2001; Drummond et al., 2005; Fenwick et al., 2001; Fenwick et al., 2004). Due to uncertainty on the monetary threshold per QALY, alternative values ranging from $€ 0$ to $€ 80,000$ were used in the cost-utility analysis (Raad voor de Volksgezondheid en Zorg [Council for Public Health and Health Care], 2006). Since the value that society would place on a unit reduction in BDI-II depression score is unknown, its NB cannot be defined (McCrone et al., 2004).

Bootstrap analyses were carried out using Microsoft Office Excel 2003. All other analyses were carried out using SPSS version 15.0.1 for Windows. 


\section{Results \\ Population}

Of the 303 eligible patients, 100 were assigned to the CCBT treatment, 103 to the TAU treatment, and 100 to the CCBT\&TAU treatment. After imputation of intermittent missings, complete data were available for 275 patients (91\%) at 6 months (CCBT: $N=91$; TAU: $N=92$; CCBT\&TAU: $N=92$ ) and 267 patients (88\%) at 12 months after baseline (CCBT: $N=88$; TAU: $N=91$; CCBT\&TAU: $N=88$ ). Baseline characteristics of patients lost to follow-up did not differ from participants who completed all assessments (de Graaf et al., in press-a).

Table 1 shows the baseline characteristics of all participants, stratified according to intervention group. The CCBT\&TAU group had lower costs on all costs categories as opposed to the CCBT and the TAU group. The TAU group showed highest costs on productivity loss, and consequently has higher total costs compared with the CCBT and CCBT\&TAU group. The CCBT group had higher baseline healthcare costs and patient and family costs compared with the other patient groups.

\section{Costs}

The mean volumes of health care use and productivity loss per patient are presented in table 2. The uncorrected costs are shown in table 3. Societal costs over the 12 months follow-up period were lowest for the CCBT group with €9,457 for CCBT, $€ 10,793$ for the CCBT\&TAU group and $€ 11,244$ for TAU. Bootstrap replications revealed a mean difference in societal costs in favour of CCBT of respectively $€ 1,784$ when compared with the TAU treatment, and $€ 1,340$ compared with CCBT\&TAU. Both health care costs and productivity costs were lowest in the CCBT group ( $€ 1,428$ and $€ 7,475)$, and highest in the TAU group ( $€ 1,912$ and $€ 8,925)$. On the other hand, patient and family costs are highest in the CCBT group (€553) and lowest in the TAU group (€408). The CCBT\&TAU group takes an intermediate position on all cost categories with $€ 1,829$ health care costs, $€ 8,425$ productivity costs, and $€ 539$ patient and family costs. About $80 \%$ of the societal costs consist of costs due to productivity loss. 
Table 1 Baseline characteristics of the sample and group differences $(N=303)^{a}$. Data are means $(S D)$ unless stated otherwise.

\begin{tabular}{|c|c|c|c|}
\hline Variable & $\begin{array}{l}\text { CCBT } \\
(N=100)\end{array}$ & $\begin{array}{l}\text { CCBT\&TAU } \\
(N=100)\end{array}$ & $\begin{array}{l}\text { TAU } \\
(N=103)\end{array}$ \\
\hline Male sex, $\mathrm{nr}(\%)$ & $48(48.0)$ & $37(37.0)$ & $46(44.7)$ \\
\hline Age $(18-65$ & $44.3(11.8)$ & $45.2(10.9)$ & $45.1(12.2)$ \\
\hline \multicolumn{4}{|l|}{ Education $^{\mathrm{b}}, \mathrm{nr}(\%)$ : } \\
\hline - Low & 18 (18.6) & $17(17.5)$ & $16(16.2)$ \\
\hline - $\quad$ Medium & $55(56.7)$ & $52(53.6)$ & $55(55.6)$ \\
\hline High & $24(24.7)$ & $28(28.9)$ & $28(28.3)$ \\
\hline Partner, yes, $\mathrm{nr}(\%)^{\mathrm{c}}$ & 72 (73.5) & $71(73.2)$ & $73(72.3)$ \\
\hline Paid job, yes, nr (\%) & $53(53.0)$ & $63(63.0)$ & $68(66.0)$ \\
\hline BDI-II & $28.2(7.7)$ & $27.4(8.2)$ & $27.9(7.5)$ \\
\hline \multicolumn{4}{|l|}{ Quality of Life: } \\
\hline - EQ-5D, UK Dolan tariff & $0.70(0.21)$ & $0.69(0.22)$ & $0.71(0.20)$ \\
\hline EQ-5D, NL Lamers tariff & $0.70(0.20)$ & $0.69(0.20)$ & $0.71(0.18)$ \\
\hline - SF-6D, UK Brazier tariff & $0.67(0.08)$ & $0.66(0.09)$ & $0.67(0.08)$ \\
\hline \multicolumn{4}{|l|}{ Costs $^{\mathrm{d}}, M[95 \% \mathrm{Cl}]^{\mathrm{e}}:$} \\
\hline Healthcare costs & $464[270,731]$ & $268[191,353]$ & $345[246,459]$ \\
\hline - Patient and family costs & $165[85,256]$ & $66[35,101]$ & $77[31,136]$ \\
\hline - Productivity costs & $2010[1375,2729]$ & $1954[1351,2605]$ & $2501[1879,3169]$ \\
\hline Societal costs & $2640[1963,3402]$ & $2288[1702,2916]$ & $2922[2300,3568]$ \\
\hline
\end{tabular}

Note. CCBT = computerised cognitive behavioural therapy; TAU = treatment as usual by a GP; BDI-II = Beck Depression Inventory II (score range 0-63); EQ-5D = EuroQol 5D (range UK Dolan tariff: $-0.59-1.00$; NL Lamers tariff: $-0.33-1.00$ ); SF-6D = Short Form 6D (range UK Brazier tariff: $0.30-1.00$ ).

a Analysis of the characteristics costs of all patients excluding the drop-outs from research questionnaires (CCBT: $N=88$; CCBT\&TAU: $N=88$; TAU: $N=91$ ) revealed similar results; ${ }^{\text {b }}$ Data unavailable: CCBT $(N=3)$; CCBT\&TAU $(N=3)$; TAU $(N=4)$; $^{\circ}$ Data unavailable: CCBT $(N=2)$; CCBT\&TAU $(N=3)$; TAU $(N=2) ;{ }^{\text {d }}$ Presented costs are costs over a 3-months period; ${ }^{e}$ The upper and lower confidence limits are the $2.5^{\text {th }}$ and $97.5^{\text {th }}$ percentile based on 5000 bootstrap replications. 


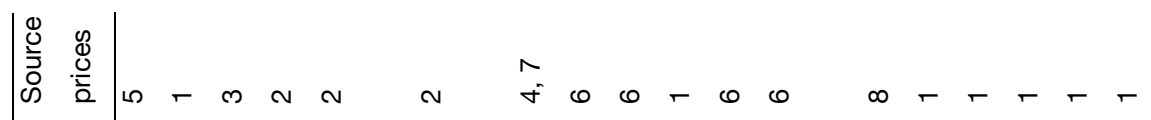

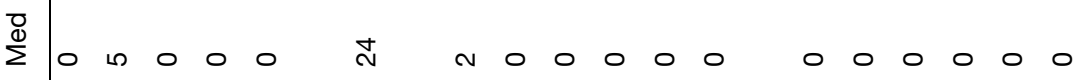 茂}

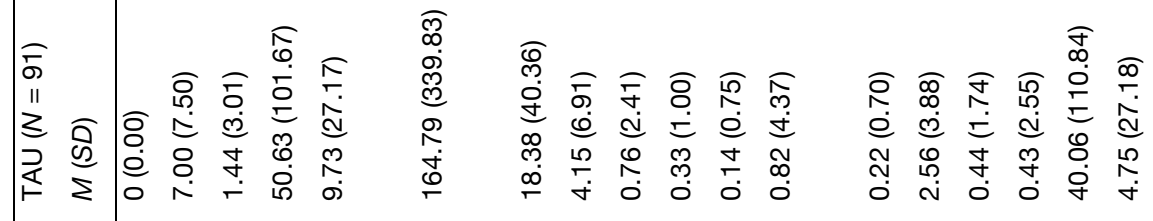
$\sum_{0}^{\infty}$

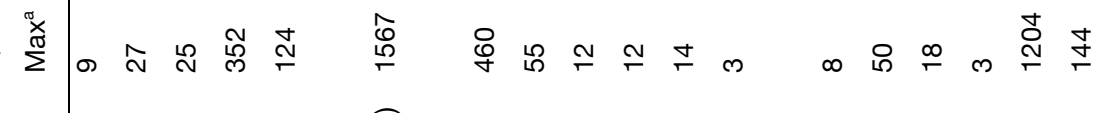

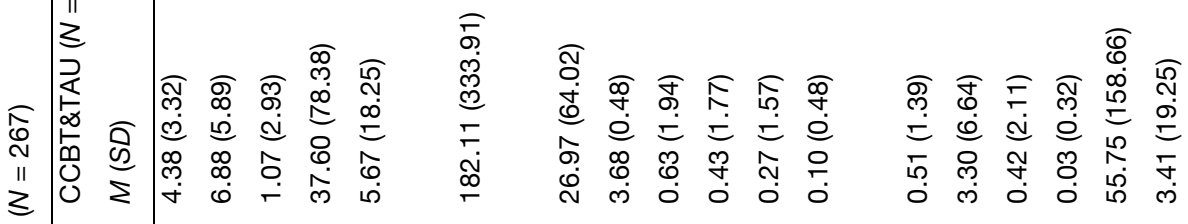

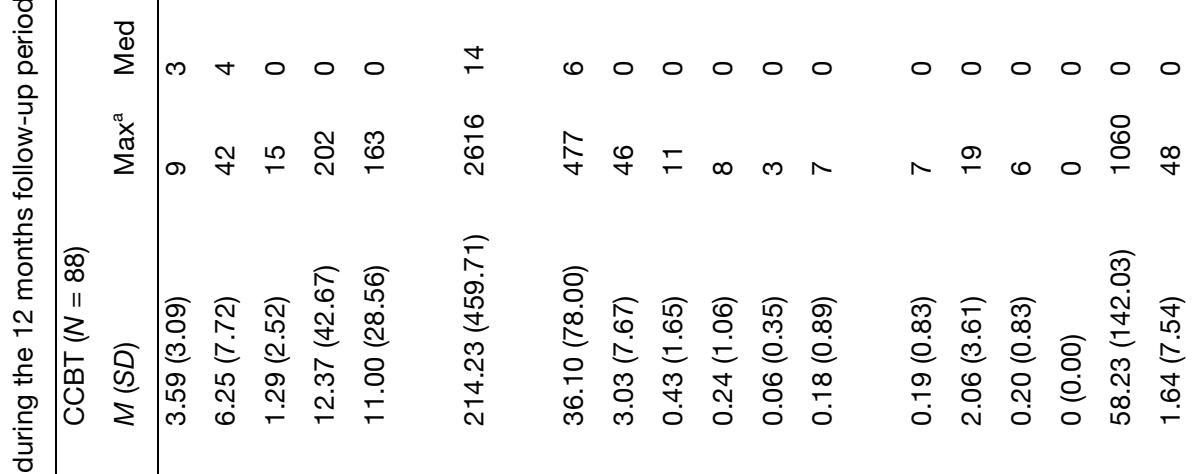

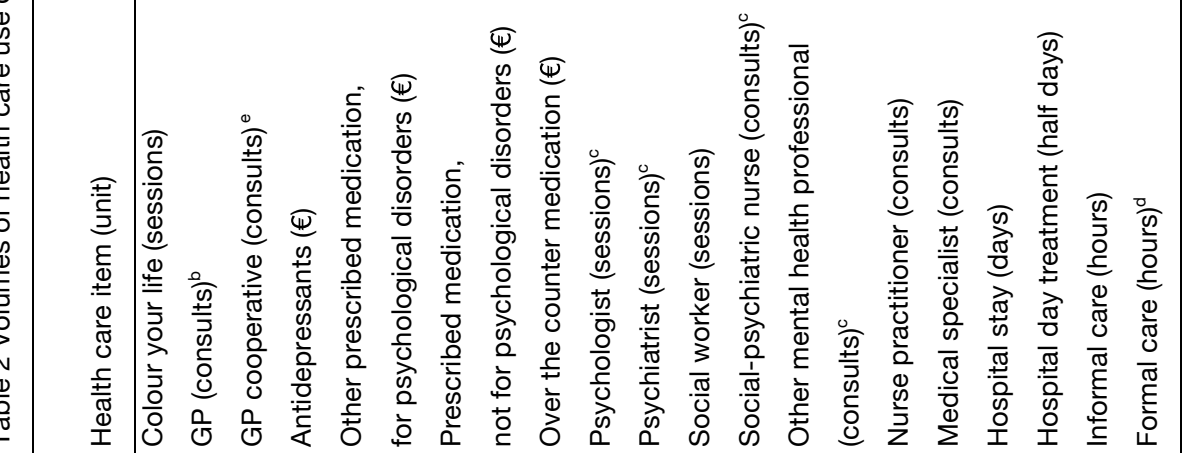




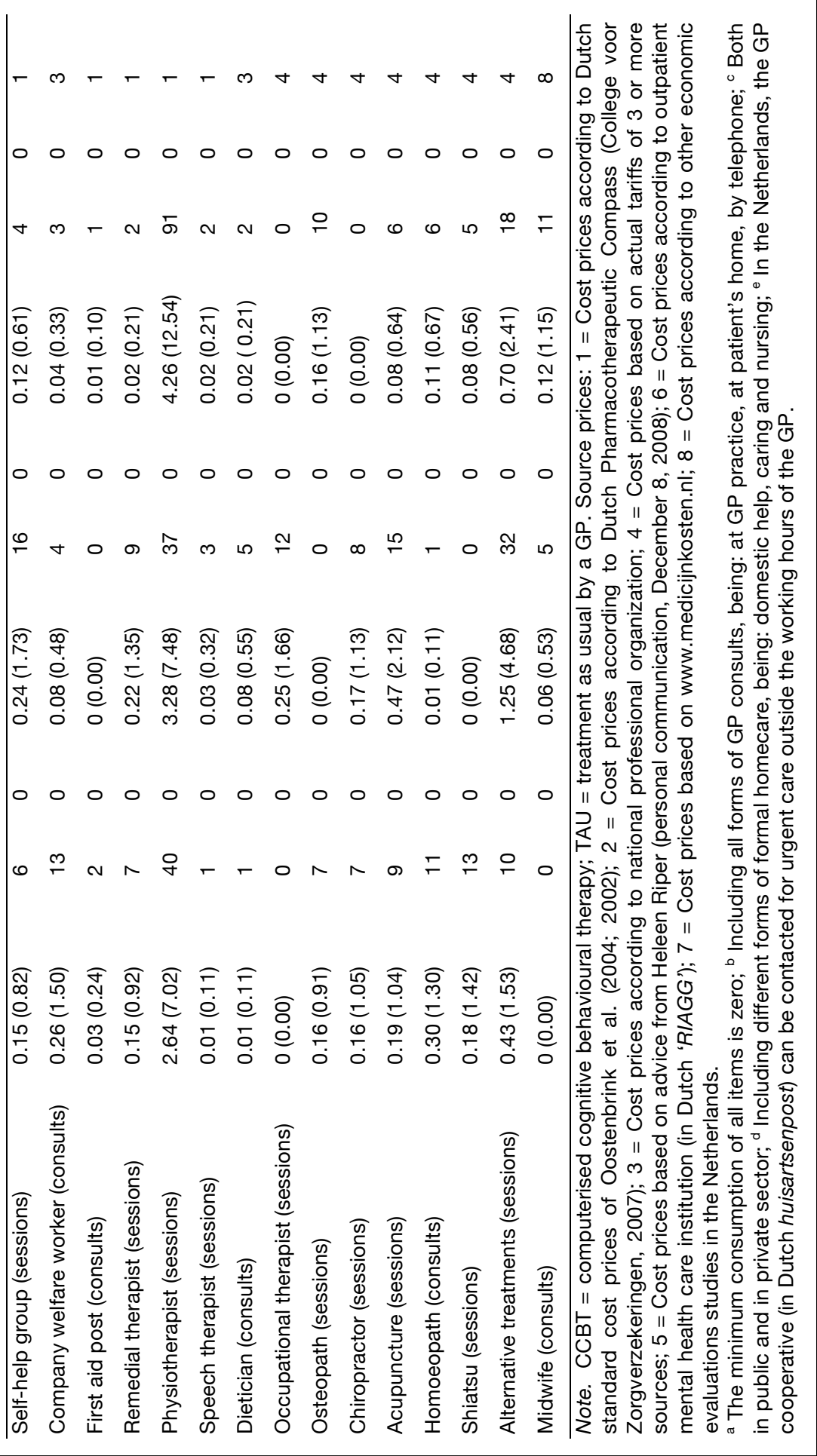


Table 3 Mean costs per cost type at 12 months after baseline $(N=267)$. Data are means.

\begin{tabular}{|c|c|c|c|}
\hline Cost type & $\begin{array}{l}\text { CCBT } \\
(N=88)\end{array}$ & $\begin{array}{l}\text { CCBT\&TAU } \\
(N=88)\end{array}$ & $\begin{array}{l}\text { TAU } \\
(N=91)\end{array}$ \\
\hline Healthcare costs $\left[95 \% \mathrm{Cl}^{\mathrm{a}}\right.$ & $1428[1121,1763]$ & $1829[1429,2261]$ & $1912[1515,2349]$ \\
\hline - $\quad$ GP care & 233 & 239 & 265 \\
\hline $\begin{array}{l}\text { - } \quad \text { Mental health care } \\
\text { specialist }\end{array}$ & 329 & 401 & 474 \\
\hline - CYL program & 41 & 40 & 0 \\
\hline - Paramedical care & 68 & 97 & 106 \\
\hline - Medical specialist care & 264 & 422 & 328 \\
\hline - Hospital care & 88 & 189 & 293 \\
\hline - Antidepressants & 12 & 38 & 51 \\
\hline $\begin{array}{l}\text { - Other prescribed and OTC } \\
\text { medication }\end{array}$ & 261 & 215 & 193 \\
\hline - Paid home care & 47 & 84 & 137 \\
\hline - Alternative healers & 78 & 100 & 63 \\
\hline - Other care & 6 & 2 & 3 \\
\hline Patient \& family costs $[95 \% \mathrm{Cl}]^{\mathrm{a}}$ & $553[321,834]$ & $539[284,859]$ & $408[236,634]$ \\
\hline - Traveling & 3 & 3 & 3 \\
\hline - Lost time & 39 & 46 & 53 \\
\hline _ Informal care & 511 & 489 & 352 \\
\hline Productivity costs $[95 \% \mathrm{Cl}]^{a}$ & $7475[5556,9430]$ & $8425[6063,10940]$ & $8925[6777,11007]$ \\
\hline - Absenteeism & 693 & 605 & 379 \\
\hline - Presenteeism & 6782 & 7820 & 8546 \\
\hline Societal costs $[95 \% \mathrm{Cl}]^{\mathrm{a}}$ & $9457[7547,11506]$ & $\begin{array}{l}10793[8412, \\
13328]\end{array}$ & $\begin{array}{l}11244[9206, \\
13419]\end{array}$ \\
\hline
\end{tabular}

Note. CCBT = computerised cognitive behavioural therapy; TAU = treatment as usual by a GP; CYL=Colour Your Life (CCBT program); OTC medication = over the counter medication.

a The upper and lower confidence limits are the $2.5^{\text {th }}$ and $97.5^{\text {th }}$ percentile based on 5000 bootstrap replications.

Table 4 shows the mean costs per patient group of the different cost scenarios used in the base case and the sensitivity analyses. In all scenarios, CCBT is the patient group with lowest mean costs per patient. In the base case analysis, costs are corrected for baseline cost differences. After regression correction for baseline differences, the CCBT group has the lowest societal costs during the 6 -months $(€ 4,462)$ and 12-months $(€ 9,092)$ follow-up period. After 6 months the mean societal costs of the TAU group $(€ 5,113)$ are a little higher compared to the costs of the CCBT\&TAU group $(€ 5,069)$. However, after 12 months the CCBT\&TAU group has the highest costs $(€ 10,534)$, while the TAU treatment takes an intermediate cost position $(€ 9,765)$ between CCBT\&TAU and CCBT. As shown in table 4, varying the costs of CYL or the valuation method of the productivity costs, implies only minor changes in costs and in differences in costs between the patient groups. Varying the cost perspective from the societal to the health care costs, implies that the TAU group has highest baseline corrected costs $(€ 1,861)$, while costs of the CCBT\&TAU group are a bit lower $(€ 1,797)$, and CCBT shows the lowest costs $(€ 1,366)$.

In the sensitivity analyses without correction for the baseline costs, the CCBT group remains the patient group with the lowest costs in all cost-varying scenarios, while the TAU group shows highest costs in all scenarios. 


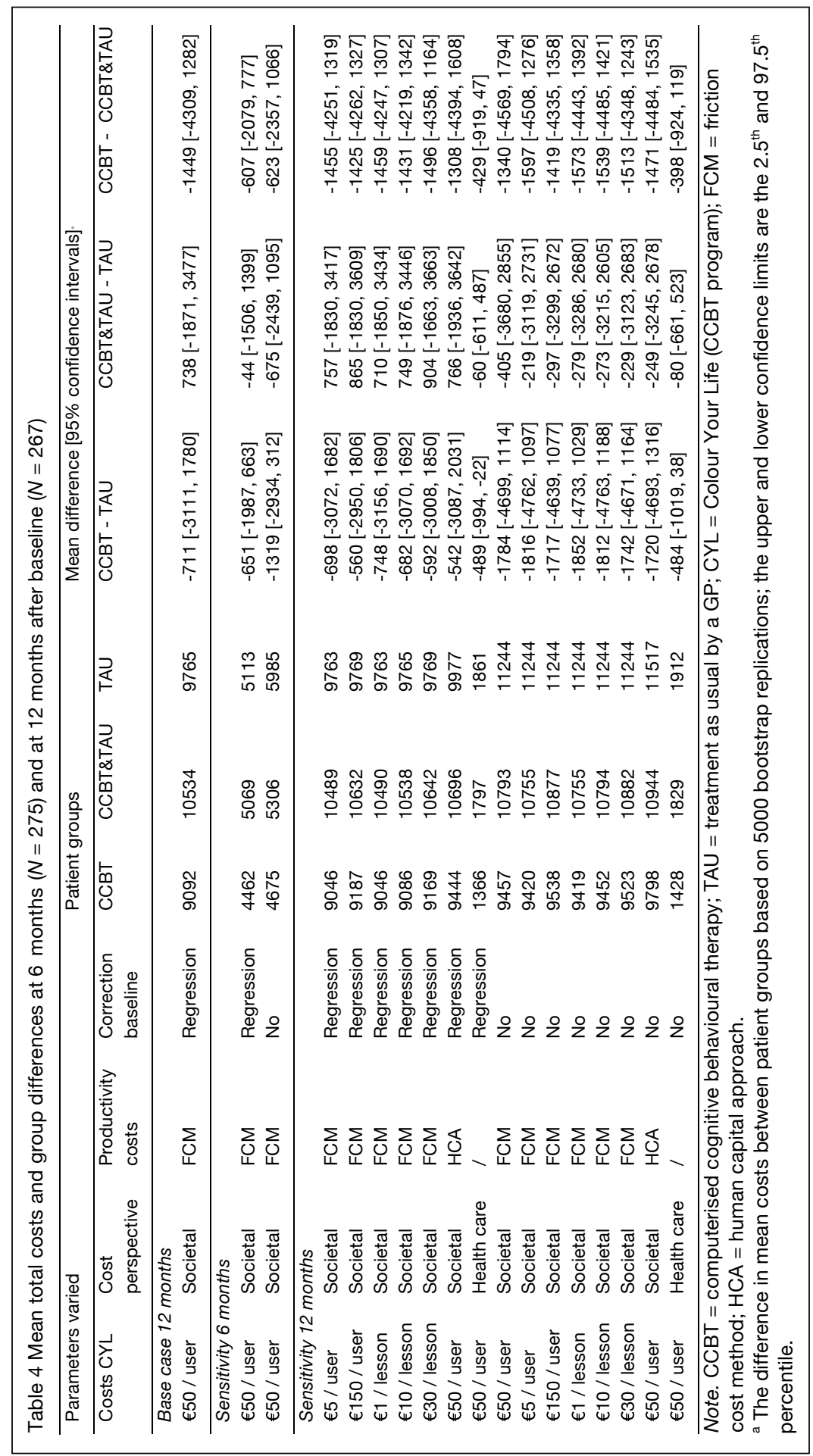




\section{Effects}

As reported in table 5, no significant group differences are found in QALYs or clinical effectiveness outcomes. In the base case analysis (baseline regression-corrected QALY using the UK-tariff to value the EQ-5D), results show a mean QALY of 0.71 for CCBT, 0.71 for CCBT\&TAU and 0.72 for TAU. The mean QALY outcomes in all three patient groups remain about 0.70 , regardless of using a baseline correction or the type of QALY measure used. There seems to be not much improvement in terms of quality of life, as the mean QALYs during the 12-months follow-up period are very similar to the mean baseline utilities of about 0.70 per patient group.

The clinical effectiveness outcome does show an improvement during the 6 and 12 months follow-up period in all three patient groups. However, the improvements in effectiveness outcomes during the follow-up period are rather minor improvements in depression (de Graaf et al., in press-a). Further details on the effectiveness outcomes are presented in table 5 . 


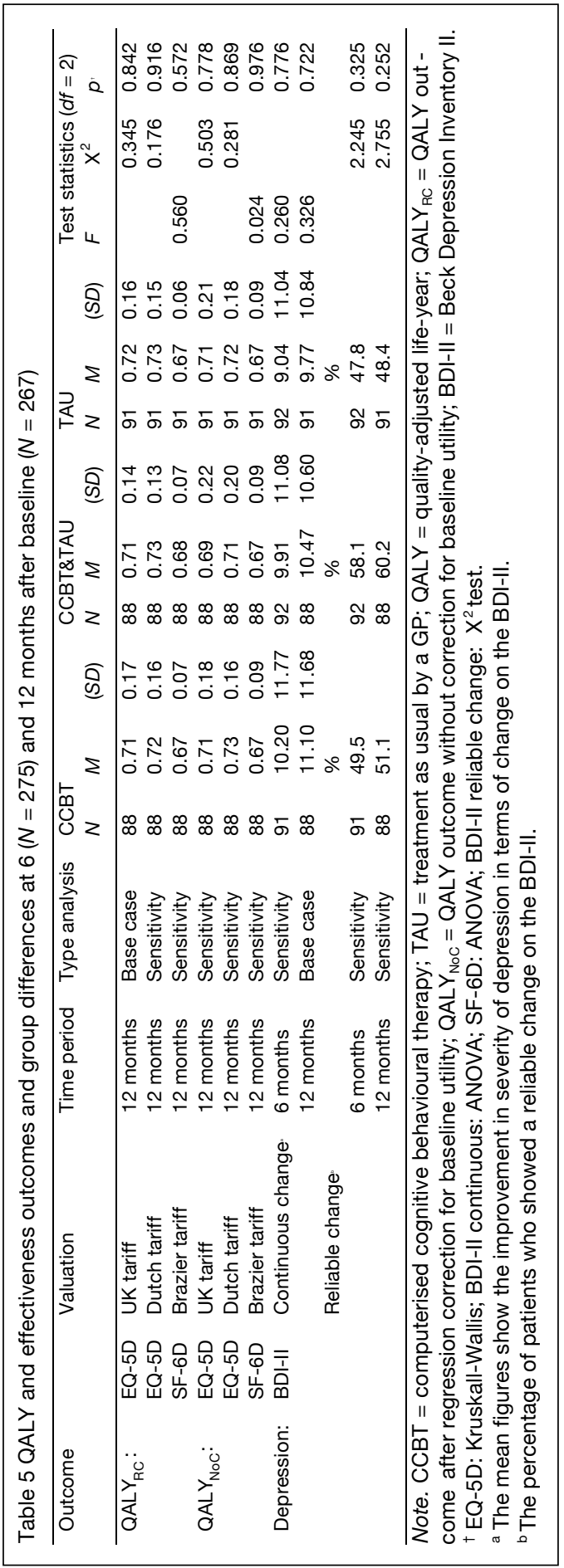




\section{Comparing costs and effects}

Figure 1 presents the CEACs of the base case cost-utility analysis with the bootstrapped baseline-corrected societal costs and EQ-5D-based QALYs. CCBT tends to be the most optimal treatment compared to CCBT\&TAU and TAU with a probability of about $65 \%$ of being the most efficient strategy at a threshold value of $€ 0$ per QALY, but diminishing towards a $40 \%$ probability when increasing the threshold value up to $€ 80,000$. Despite the $25 \%$ probability of TAU being the most efficient strategy at a threshold value of $€ 0$ per QALY, its probability increases up to about $40 \%$ when society's willingness to pay increases up to $€ 80,000$ per QALY, and consequently the choice between TAU and CCBT becomes almost indifferent at a threshold of $€ 80,000$. The CCBT\&TAU treatment has the lowest chance of being the most efficient strategy, with probabilities varying from $10 \%$ to $20 \%$ at the presented threshold values per QALY.

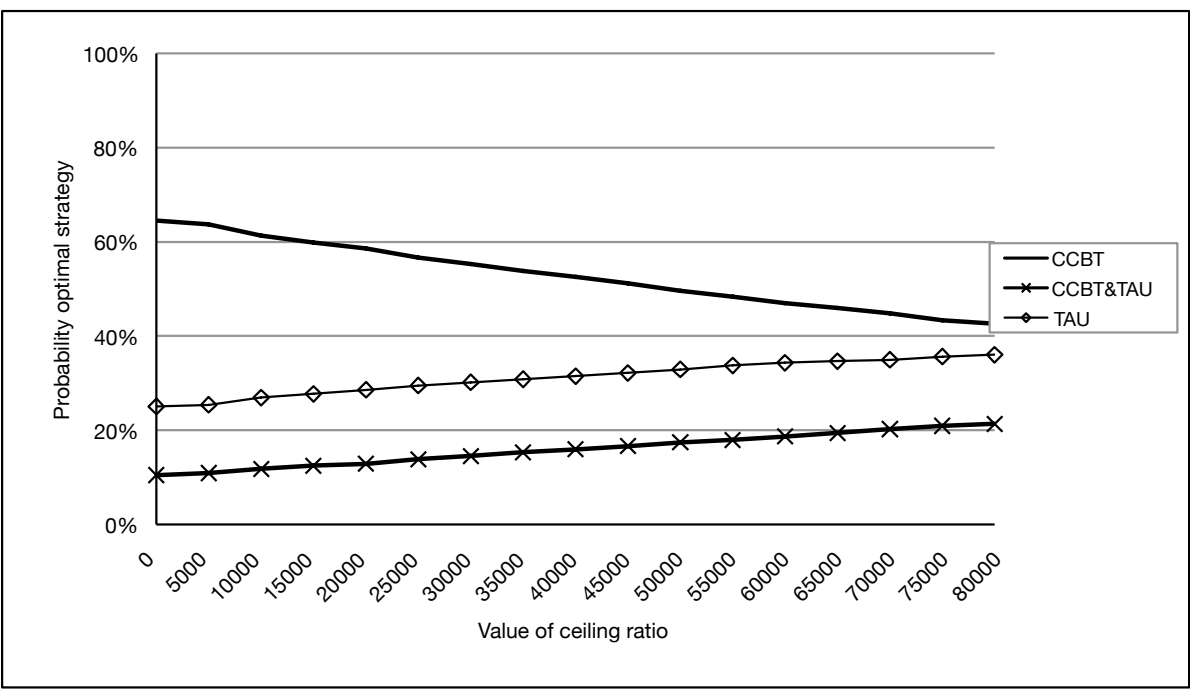

Figure 1 Base case analysis. Cost-effectiveness acceptability curves of the cost-utility at 12 months follow-up

Note. CCBT = computerised cognitive behavioural therapy; TAU = treatment as usual by a GP; costs = societal costs after regression correction for baseline costs; utility = quality-adjusted life-year (EQ-5D, UK tariff) after regression correction for baseline utilities. 
Figure 2 shows the CEACs for the base case cost-effectiveness analysis at 12 months follow-up using point changes on the BDI-II scale as outcome. As there is no guideline on the threshold value per point improvement on the BDI-II, the value of the ceiling ratio is varied up to an amount of $€ 40,000$ where the probabilities per treatment remain rather stable for higher thresholds. The CCBT treatment shows highest probabilities of about $60 \%$ of being the most efficient strategy, regardless of the threshold value for an improvement on the BDI-II scale. If one is willing to pay at least $€ 5,000$ per point improvement on the BDI-II scale, the TAU treatment is less favoured with probabilities about $10 \%$ of being the most optimal strategy, while CCBT\&TAU shows probabilities about $30 \%$. Below the threshold value of $€ 5,000$, TAU has a probability between 25 to $13 \%$, while the CCBT\&TAU treatment shows increasing probabilities of about $10 \%$ to $20 \%$.

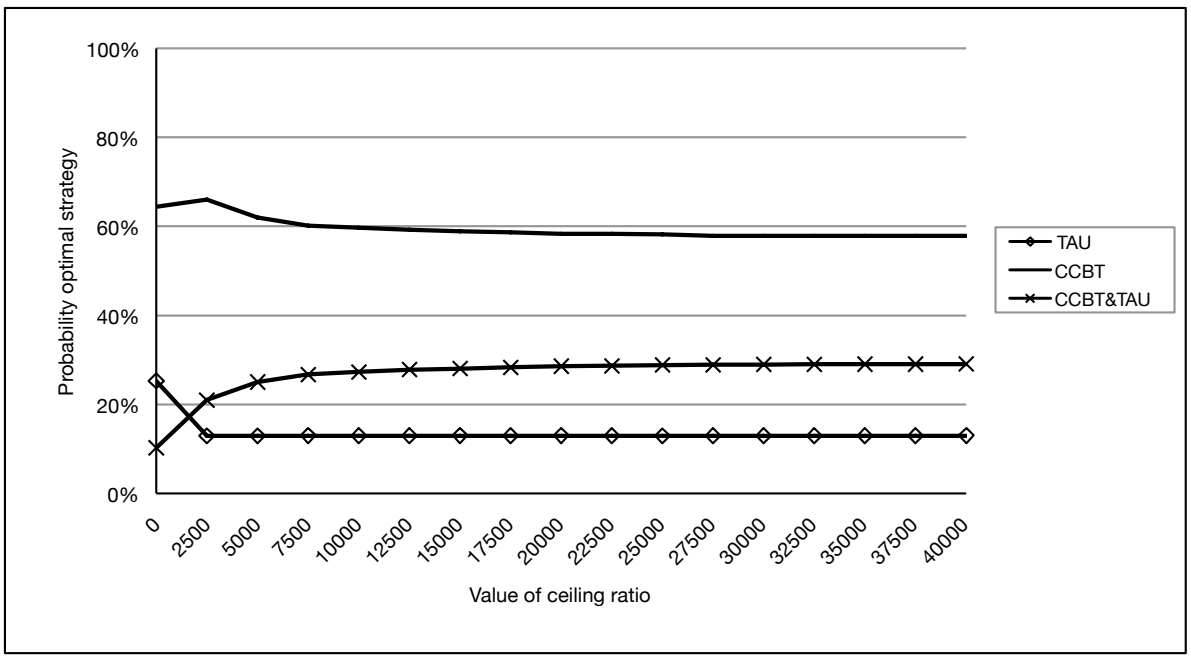

Figure 2 Base case analysis. Cost-effectiveness acceptability curves of the cost-effectiveness at 12 months follow-up

Note. CCBT = computerised cognitive behavioural therapy; TAU = treatment as usual by a GP; costs = societal costs after regression correction for baseline costs; effectiveness = points improvement on the Beck Depression Inventory II. 


\section{Sensitivity analyses}

Varying the outcome parameter of the base-case cost-utility scenario to either the regression-corrected QALY valued by the Dutch EQ-5D tariff or the SF-6D revealed similar results. Varying the regression-corrected societal costs by different cost calculations of CYL, or the productivity costs to the human capital approach, did not show much difference from the base case analysis with QALY as outcome measure. CCBT is still the most favourable treatment in terms of cost-utility. However, as a consequence of not using baseline corrections, the TAU treatment turns out to have the lowest probability of being the most optimal choice. In this scenario, CCBT has a 70 to $80 \%$ probability of being the most efficient treatment given the varying threshold values up to $€ 80,000$, while CCBT\&TAU and TAU each have a probability about $10-20 \%$.

The sensitivity analysis with most deviation from the base case cost-utility analysis is the scenario in which the health care cost perspective is used. Figure 3 shows CCBT is the most efficient strategy for treatment at the lower threshold values. But when increasing the ceiling on the value per QALY, the choice between the three treatment strategies becomes indifferent as all curves approach a probability of $30 \%$.

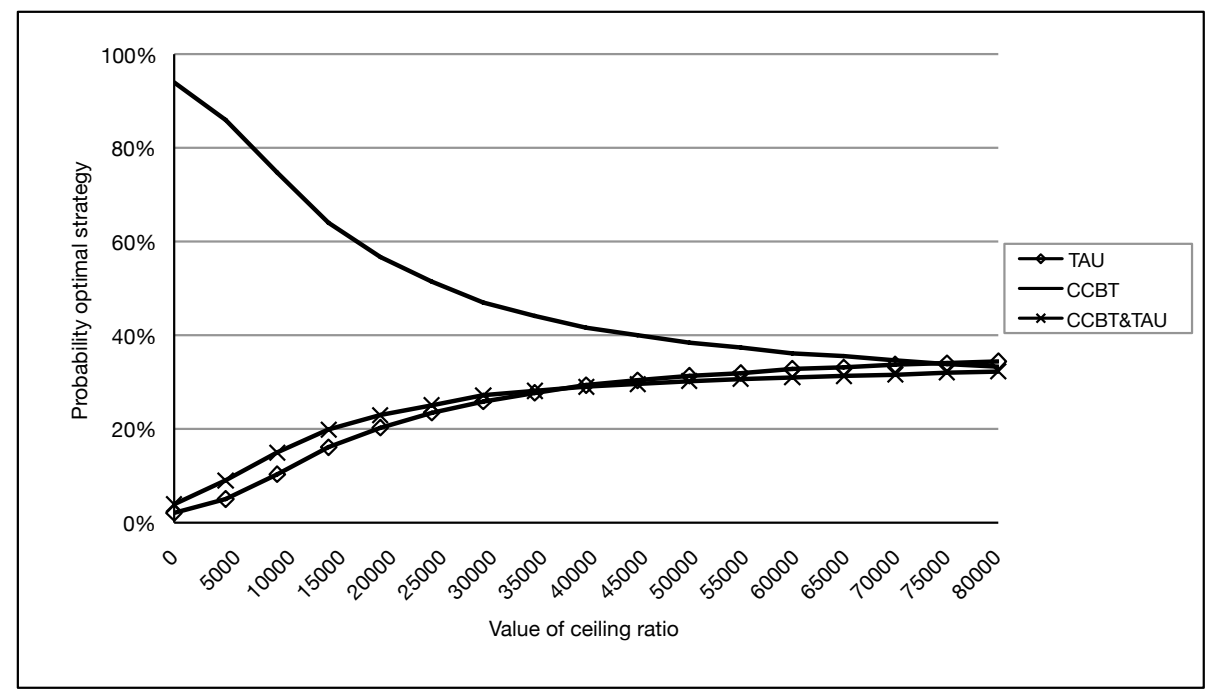

Figure 3 Sensitivity analysis. Cost-effectiveness acceptability curves of the cost-utility with healthcare costs at 12 months follow-up

Note. CCBT = computerised cognitive behavioural therapy; TAU = treatment as usual by a GP; costs = healthcare costs after regression correction for baseline costs; utility = quality-adjusted life-year (EQ$5 \mathrm{D}$, UK tariff) after regression correction for baseline utilities. 
When using clinical effectiveness as outcome measure, most of the sensitivity analyses resulted in minor differences from the CEACs of the base case analysis at 12 months follow-up. The variations in societal cost calculation by using different cost for CYL, varying the productivity costs to the human capital approach, varying the use of baseline corrected costs, varying the cost perspective to the health care perspective or varying the time period by 6 months time period resulted in similar CEACs as the base case analysis at 12 months follow-up. Only varying the outcome measure into the reliable BDI-II change showed differences from the base case analysis in which the costs were related to the absolute change on the BDI-II. As presented in figure 4, $\mathrm{CCBT}$ is still the favoured treatment at the lower threshold values. However, the curve of CCBT\&TAU shows a steeper increase. From a threshold value of $€ 7,000$ onwards, CCBT\&TAU outperforms CCBT up to $80 \%$ chance of being the most optimal strategy, while CCBT decreases to $20 \%$ at higher threshold values for a patient with a reliable BDI-Il change.

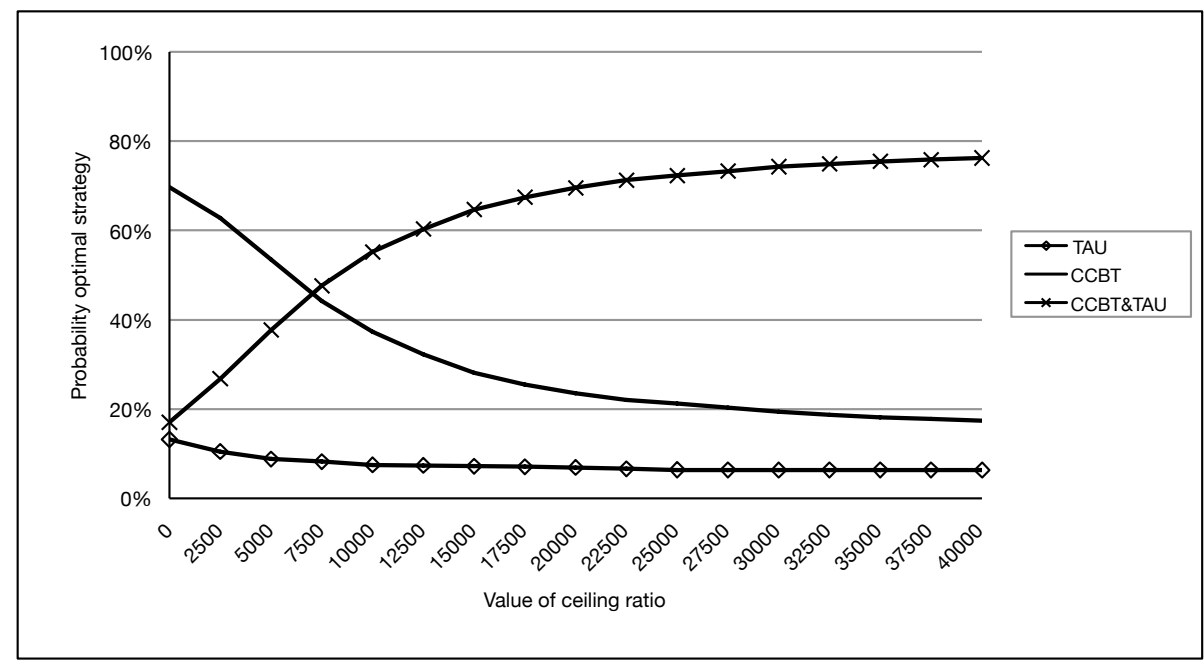

Figure 4 Sensitivity analysis. Cost-effectiveness acceptability curves of the reliable change on the BDIII at 12 months

Note. CCBT = computerised cognitive behavioural therapy; TAU = treatment as usual by a GP; BDI-II= Beck Depression Inventory II; costs = societal costs after regression correction for baseline costs; effectiveness $=$ reliable change (i.e. 9 points improvement) on BDI-II. 


\section{Discussion}

\section{Main results}

At first glance, CCBT seems to be the most efficient treatment strategy, as in most cost-effectiveness and cost-utility scenarios CCBT has the highest probability of being the most optimal treatment choice.

In all cost-utility analyses, the CCBT treatment turned out to be the most efficient strategy for treatment. The mean societal costs were lowest in this group, while the cost-utility CEACs showed that CCBT has the highest probability of being the most efficient treatment strategy compared with CCBT\&TAU and TAU. However, when increasing the threshold value for an additional QALY, the difference between CCBT versus TAU and CCBT\&TAU decreases and in some analyses tends to indifference in the choice between the three treatments in terms of cost-utility.

With probabilities of about $60 \%$ of CCBT being the most efficient treatment choice, the cost-effectiveness analysis using the absolute change on the BDI-II also shows a preference for the CCBT treatment versus TAU and CCBT\&TAU. However, when evaluating the reliable change on the BDI-II, the CCBT\&TAU treatment outperforms CCBT at threshold values above $€ 7,000$ per patient achieving reliable change. Despite the outcomes of the economic evaluation, there are no statistically significant differences in effectiveness or QALY outcomes, and all three treatments do not seem to achieve much improvement in depression or quality of life during the 12-month follow-up period. However, changing the BDI-Il outcome from absolute change to reliable change does have an impact on the cost-effectiveness outcomes. While the BDIII decreases on average about 10 points per patient in all groups, using the cut-off score of 9 for the reliable change results in $60 \%$ of CCBT\&TAU patients with reliable change, compared with about $51 \%$ and $48 \%$ for CCBT and TAU. Although not significant, this difference in reliable change on the BDI-II does seem to be relevant and in favour of the CCBT\&TAU treatment. Given the high depressive severity of our sample, the differences in reliable change on the BDI-II might not seem surprising. For more severely depressed patients, combination treatments have been found to be superior to single treatments (Friedman et al., 2004; Pampallona et al., 2004).

\section{Previous studies}

Until now, only one study has been published on the cost-effectiveness of CCBT for depression. McCrone et al. (2004) performed a randomised trial in which supported CCBT was compared to usual care for anxiety and depression. In this study, the healthcare costs of CCBT were a little higher as opposed to the healthcare costs of TAU, while our study showed the reverse. This might be explained by differences in the type of intervention. In the study of McCrone, CCBT was supported and took place at the GP practice, consequently involving more healthcare costs. In accordance with our study, productivity costs of CCBT were lower compared with the productivity costs of TAU, resulting in TAU being more expensive compared with CCBT.

Contrary to our study, CCBT was clinically superior to TAU and showed higher effect sizes in the McCrone study (de Graaf et al., in press-a; McCrone et al., 2004). In their study, McCrone found that if society is willing to pay only a small amount for increased effectiveness, CCBT has a higher chance of being cost-effective compared with the TAU treatment. Both the McCrone and our study thus seem to be in favour of the CCBT treatment versus TAU in terms of cost-effectiveness. 


\section{Methodological considerations}

In this study, we made several assumptions on the calculation and valuation of costs. First of all, the costs of CYL are estimates based on the actual costs of the program, and we decided to calculate costs only for those participants who at least once logged in to the program. Different views on the calculation of CYL costs per patient might exist. At the one hand, one might expect that a patient will have to pay for using the program, or for the time duration using the program. At the other hand, one may also argue that in real-life a patient would have to pay for receiving a login code for the program, regardless of his or her use of CYL. Moreover, the time costs of patients who followed CYL might have been underestimated. The time spent on CYL was based on the computer-registered login time, and does not include the time a patient might have spent on CYL by reflecting on the lessons' content or doing homework tasks offline. On the other hand, the computer-registrations might as well give an overestimation of time spent on CYL as one might as well do other things during the time logged in on CYL. Moreover, we opted not to account for costs of a computer and/or Internet. Reason for this is that patients who participated in the trial were required to have a computer at home with a non-dial-up Internet connection. Consequently, participating in the CYL program should not lead to any additional expenditure on computer or Internet. However, this also means that our study sample is limited to the population of Internet and computer users.

In the recording of cost data, one important difference is that the costs of CYL are based on the objective computer-registered data, while costs of other health care items are based on the patient-reported care consumption. The latter method might as well have lead to an over- or underestimations of the health care use, as selfreports by patients can be vulnerable to recall error (van den Brink et al., 2004).

In the base case analysis, we used a regression correction for the baseline costs and QALYs. Although utilities did not differ significantly among the patient groups, it is likely that the patient's baseline utility is highly correlated with the QALY outcome. As argued by Manca et al. (2005), controlling for baseline utility is advised to be standard practice in economic evaluations. As shown in our sensitivity analyses, the baseline correction did affect the outcomes of the costs and cost-effectiveness and cost-utility analyses. In the base case analysis, CCBT\&TAU is the patient group with highest costs during the 12 months follow-up (CCBT\&TAU €10,534; TAU €9,765; CCBT €9,092), while in the sensitivity analysis without correction, it is the TAU treatment that shows the highest mean societal costs per patient (TAU €11,244; CCBT\&TAU €10,793; CCBT $€ 9,457)$. Although CCBT remained the treatment with the highest probability of being most cost-effective, its probability in the base case cost-utility analysis was much lower (about $65-40 \%$ ) than in the sensitivity cost-utility analysis without correction (70-80\%). The TAU group has a probability of about $25 \%$ to $40 \%$ in the base case cost-utility analysis, while in the sensitivity analysis its probability is only about 10 to $20 \%$. These differences in corrected versus uncorrected costs and outcomes stress the importance of taking baseline outcomes into account in economic evaluations.

In our cost-utility analysis, we interpreted the results at varying monetary threshold values of a QALY up to $€ 80,000$. However, the exact threshold value is unknown, and there are no exact guidelines available in the Netherlands. Several studies on the threshold suggested values of $€ 10,000$ to $€ 27,000$ per QALY (Gyrd-Hansen, 2003; King et al., 2005; Laupacis et al., 1992), while in general $€ 18,000$ is accepted as the 
threshold value per QALY for preventive care in the Netherlands (Casparie et al., 1998; Raad voor de Volksgezondheid en Zorg [Council for Public Health and Health Care], 2006). However, the Dutch Council for Public Health and Health Care (2006) recommends relating the threshold of the costs of a QALY to the burden of disease, with a limit of $€ 80,000$ per QALY at a maximum burden of disease of 1.0. Despite the absence of a clear guideline, we assume that the chosen range of cost-utility thresholds in our study is broad enough to capture the relevant threshold values.

A last remark concerns the scope of costs included in the analysis. Because of the broad impact depression can have on a patient's life, we chose to include all health-related healthcare, productivity and patient and family costs in the analysis. However, since the costs are not limited to depression-related costs, resulting costs are probably an over-estimation of the costs due to depression.

Despite the assumptions mentioned above, we believe that one of the strengths of our study is that the main findings were rather robust. In the sensitivity analyses, we tried to capture the uncertainty in the assumptions. Both the base-case analysis and the sensitivity cost-utility analyses indicated a preference for CCBT at a considerable range of cost-effectiveness and cost-utility thresholds.

\section{Implications of the study characteristics}

In our sample, the adherence to treatment as well as the clinical effectiveness and change in quality of life were rather low (de Graaf et al., in press-a). In this study, there was no control group receiving no care. One of the questions is whether the changes in clinical effectiveness or quality of life of our participants can be attributed to the treatment received - especially given the low adherence rates - or whether they reflect the natural course of depression. In the absence of a no-treatment group, it is not clear whether the participants' depression and QALY changes differ from depressed people who do not receive treatment during a 12-month follow-up period. The low adherence to treatment could explain the small differences in costs between the three treatment groups. Possible explanations for the low effect of treatment and the low adherence rate have been suggested elsewhere (de Graaf et al., in press-a).

Moreover, BDI-II scores at baseline in our sample were higher than generally seen in depressed primary care patients (Vuorilehto et al., 2007). The interventions offered in our study might have been less suited for this patient group. A more intensive and/or supported treatment form might have been justified for this population, and might have generated better outcomes in terms of QALY and clinical effectiveness.

\section{Conclusion}

Differences in costs and outcomes of the three treatment groups are rather small. The cost-utility and cost-effectiveness analyses tend to be in favour of the CCBT treatment as opposed to the TAU and CCBT\&TAU treatment. However, the low adherence rate and modest improvements in depression and quality of life outcomes, combined with the severity of depression of our study sample, raises questions on the suitability of this unsupported treatment form for this type of depressed patients. 


\section{Acknowledgements}

We thank Annie Hendriks and Greet Kellens for their assistance during the study and Rosanne Janssen for the development of the infrastructure for online data-collection. Thanks to Fons Kessels, Thea van Asselt, Frenk Peeters, John Pletzers, Filip Smit, Marc Koopmanschap, and Jorne Grolleman for their help on specific questions. The trial is financed by ZonMw (Netherlands Organisation for Health Research and Development; project number 945-04-417), research institute EPP and research institute CAPHRI. Municipalities Eijsden, Meerssen, Sittard-Geleen, Valkenburg and Maastricht sponsored the study. 



\section{Chapter 8}

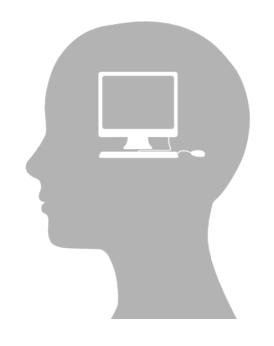

\section{Use and acceptability of}

unsupported online

computerised cognitive

behavioural therapy for

depression and associations

with clinical outcome

L.E. de Graaf, M.J.H. Huibers, H. Riper, S.A.H. Gerhards \& A. Arntz 


\section{Abstract}

Background: In a recent randomised trial, we were unable to confirm the previously reported high effectiveness of computerised cognitive behavioural therapy (CCBT) for depression. Therefore, the aim of the current study was to have a closer look at usage and acceptability (i.e. expectancy, credibility, and satisfaction) of the intervention, and how these were related to treatment outcome (i.e. reduction in depressive symptoms and dysfunctional beliefs).

Methods: Depressed participants $(N=200)$ were given login codes for unsupported online CCBT, named Colour Your Life (CYL). A track-and-trace system tracked which CYL components were used. We had a 9-month follow-up period.

Results: Uptake of CYL was sufficient, but dropout was high. Many usage indices were positively associated with short-term depressive improvement, whereas only homework was related to long-term improvement. CYL adherence was not associated with reduction in dysfunctional beliefs. Pre-treatment acceptability was good and expectancy could predict long-term, but not short-term outcome.

Limitations: Associations between use of CYL and improvement are merely correlational. Our sample was too depressed in relation to the scope of the intervention. We relied on online self-report measures. Analyses were exploratory in nature.

Conclusions: Although CCBT might be a feasible and acceptable treatment for depression, means to improve treatment adherence are needed for moderately to severely depressed individuals. 


\section{Introduction}

Depression is a common complaint in the general population (Bijl et al., 1998). Although depression can be treated effectively with cognitive behavioural therapy (CBT) (e.g. see Butler et al., 2006; Hollon et al., 2006), many depressed individuals remain untreated (Hirschfeld et al., 1997). An effective, acceptable, and feasible solution might be computerised CBT (CCBT) (Kaltenthaler et al., 2006; Kaltenthaler et al., 2008a), which can vary greatly in terms of technologies used and amount of additional support. Especially unsupported websites of CCBT can have high potential in improving access to care. Such interventions can be effective (Spek et al., 2007b). In a recent randomised trial (de Graaf et al., in press-a), we evaluated the effectiveness of unsupported online CCBT (i.e. Colour Your Life/CYL) for depression in primary care compared with usual GP care (TAU) and a combination of both treatments. In contrast to previous studies, CCBT did not outperform treatment as usual and the combination treatment had no additional effects either in both the intention-to-treat and perprotocol analysis. The between-group effect size $(d=0.20)$ was smaller than found in previous studies, e.g. 0.55 for a similar intervention (Spek et al., 2007b) and 0.65 for supported CCBT in primary care (Proudfoot et al., 2004). We suggested that the intensity of unsupported online CCBT might have been too low our study population of moderately to severely depressed patients.

Since we were unable to confirm the previously reported high effectiveness of CCBT, it might especially be interesting to have a closer look at how the intervention was used. Knowledge on the usage of computerised interventions is important to optimise and implement CCBT in actual practice, and can be seen as an indicator for the feasibility of the intervention. Previous studies reporting on the usage of unsupported, online CCBT for depression have generally shown high treatment dropout rates (e.g. see Christensen et al., 2006a; Christensen et al., 2004b; Clarke et al., 2002; Kaltenthaler et al., 2008a; Spek et al., 2007b). However, we know little about predictors of the uptake of computerised self-help. Factors related to the ability to use computers and work independently, such as education and depressive severity, might be important to consider (see McKendree-Smith et al., 2003). Furthermore, the level of usage of CCBT has rarely been associated with clinical outcome in depression. Some studies showed that completion of more site material was associated with better psychological outcomes for depression (Christensen et al., 2006a; Osgood-Hynes et al., 1998), obsessive-compulsive disorder (Marks et al., 1998b), and eating disorders (Manwaring et al., 2008). Taken together, little is known regarding factors that are associated with the uptake of and outcome in CCBT for depression apart from lack of support, which leads to dropouts (see Eysenbach, 2005).

Another aspect that might be important to consider when evaluating the effects of a new intervention is the acceptability by patients before and after treatment (e.g. expectancy, credibility, and satisfaction), which has been rarely assessed in research (Kaltenthaler et al., 2008b). Pre-treatment expectations are not only important in predicting psychotherapy outcome (e.g. see Goossens et al., 2005; Greenberg et al., 2006; Ilardi \& Craighead, 1994), but can also predict engagement in therapy (Carr et al., 2001; Meyer et al., 2002). We are aware of only few studies that related pretreatment acceptability in CCBT to adherence or treatment outcome. For instance, completion of computerised exposure sessions was associated with high baseline motivation in obsessive-compulsive disorder (Marks et al., 1998b). In another study, 
bulimic patients who did not take up CCBT had lower expectations of the usefulness of the intervention for themselves (Murray et al., 2003). In a computer-aided treatment for depression, the strongest predictor of treatment response was how logical the treatment seemed to patients (Osgood-Hynes et al., 1998). Regarding post-treatment acceptability, a recent review of Kaltenthaler et al. (2008b) showed that in general beliefs about CCBT for depression were positive, although this was reported for treatment completers giving a biased view of patients' perceptions of the treatment. Marks and colleagues (2003) showed that depressed and anxious patients were fairly satisfied with various computerised CBT systems. In a study on phobic and panic disorder, post-treatment satisfaction with online CCBT with support correlated positively with the outcome (Schneider et al., 2005).

Given all of the above, the aim of the current study is threefold. First, we will describe how online, unsupported CCBT (i.e. Colour Your Life) was used and how acceptable the intervention was to patients at baseline and after treatment, including those who did not comply with the treatment protocol. Second, we will investigate factors that might predict uptake of CCBT (i.e. completion of the first session). Third, we will investigate whether use of CCBT and acceptability markers are related to reduction in depressive severity. Since it has been hypothesised that CBT reduces depression by changing dysfunctional cognitions (Beck, 1983), dysfunctional attitudes will also be used as an outcome variable in an additional analysis. We hypothesise that frequent use of CCBT and positive expectations will be associated with better outcomes regarding depressive symptoms and depressogenic cognitions. 


\section{Method}

\section{Design}

The design of the study is a randomised trial with three conditions: (a) unsupported online CCBT, (b) TAU by a GP, and (c) CCBT and TAU combined. The Medical and Ethical Committee of Maastricht University approved the study protocol. The study is registered at the Netherlands Trial Register, part of the Dutch Cochrane Centre (ISRCTN47481236). Full details of the study method have been described elsewhere (de Graaf et al., 2008a).

\section{Participants}

Participants were recruited in the general population by means of a large-scale Internet-based screening in the South of the Netherlands. Potentially eligible participants were invited to visit the research centre for an intake where final eligibility was assessed. Participants were eligible if they met the following criteria: age 18 to 65 ; access to the Internet at home; at least mild to moderate depressive complaints (BDI-II score $\geq 16$ ); duration of depressive complaints 3 months or more; no current psychological treatment for depression; no continuous antidepressant treatment for at least 3 months prior to entry; fluent in Dutch language; no alcohol and/or drug dependence; and no severe psychiatric co-morbidity (e.g. psychotic disorders). For the purpose of the present study, only participants allocated to CCBT alone or the combination treatment were included in the analyses $(N=200)$.

\section{Measures}

\section{Composite International Diagnostic Interview}

To determine DSM-III-R axis I diagnoses the computerised CIDI (CIDI-auto) was used, which has acceptable reliability and validity (Robins et al., 1988; Wittchen, 1994).

\section{Beck Depression Inventory II}

The BDI-II measures the severity of depression with high scores indicating severe depression (range 0-63). There has been consistent support for its construct validity and reliability in various samples (Beck et al., 1996; van der Does, 2002).

\section{Dysfunctional Attitude Scale form A}

The intensity of dysfunctional beliefs was assessed with the DAS-A (Weissman \& Beck, 1978). The Dutch version of the DAS-A has good psychometric properties (Raes et al., 2005). We used a 17-item version, which demonstrated good reliability $(\alpha=.91)$ and convergent construct validity (de Graaf et al., in press-b). The higher the score, the more dysfunctional attitudes an individual reports (range $17-119$ ).

\section{Credibility/expectancy questionnaire}

We translated the English CEQ (Devilly \& Borkovec, 2000) into Dutch. The CEQ consists of six items and was used to measure treatment expectancy and rationale credibility. The credibility scale consists of three items: (1) at this point, how logical does the treatment offered to you seem; (2) at this point, how successfully do you think this treatment will be in reducing your symptoms; (3) how confident would you be in recommending this treatment to a friend who experiences similar symptoms. The expectancy scale consists of the following three items: (1) by the end of the treatment, how 
much improvement in your symptoms do you think will occur; (2) at this point, how much do you really feel that the treatment will help you to reduce your symptoms; (3) by the end of treatment, how much improvement in your symptoms do you really feel will occur. In our translation, we modified the answer scales of two questions (range $0-100 \%$ ) such that all items were to be rated on a 9-point Likert scale, ranging from 1 (e.g. "not at all logical") to 9 (e.g. "very logical"). The score for each subscale is the sum of the items (score range per subscale $3-27$ ). The CEQ proved to be a reliable measure with a stable factor structure across different samples (Devilly \& Borkovec, $2000)$. The internal consistency of our translation was acceptable for both credibility $(a=.82)$ and expectancy $(a=.90)$.

\section{Expectancy question}

Patients rated their expectancy also on a single item (i.e. what do you expect of the treatment or combination of treatments?) consisting of five answer options: (1) the treatment will cure my depression, (2) after the treatment I will be less depressed, (3) I will learn how to cope with my depression, (4) I will learn to accept my depression, and (5) the treatment will not affect my depression.

\section{Satisfaction with treatment allocation}

Patients were asked how satisfied they were with the treatment they were allocated to (1 = yes; 2 = neutral; 3 = no).

\section{Evaluation questionnaire}

The evaluation questionnaire consisted of seven statements: (1) I was satisfied with the treatment; (2) the treatment was easy; (3) I feel that the treatment will have an enduring effect; (4) I would recommend the treatment to others; (5) the treatment was useful; (6) the treatment was impersonal; (7) the treatment was good. Each statement was rated on a 5 -point scale ( $1=$ completely agree; $5=$ completely disagree).

\section{Track and trace}

Track and trace is a system that tracks which aspects of CYL were used and for how long. The following variables were used: total time spent, time spent per login, number of visits to various aspects of the program (i.e. sessions, homework assignments, forum, and mood diary), number of finished sessions (range 0-9), and number of completed homework assignments (range 0-13).

\section{Procedure}

After written informed consent was obtained, participants were randomly allocated to one of the three primary care interventions. Baseline assessment took place at the research centre before randomisation. Patients were interviewed using the CIDI and completed questionnaires on a computer (i.e. demographic variables, BDI-II, and DAS-A). At baseline, but after randomisation, the CEQ, the expectancy question, and the satisfaction question were administered. Follow-up assessments took place at home via the Internet. The BDI-II and the DAS-A were administered again at three and nine months of follow-up. The evaluation questionnaire was completed at three months. 


\section{Intervention}

The CCBT program, named Colour Your Life (CYL), is an online, multimedia, interactive, self-help computer program for depression (Riper \& Kramer, 2004). Readability for several educational levels was taken into account. The combination of text, audio, and video makes it potentially suitable for a broad public. CYL is based on the principles of CBT and on the Dutch version of the 'coping with depression course' of Lewinsohn (Cuijpers et al., 1995). The program consists of eight sessions and a ninth booster session. The duration of sessions can vary among users. At the end of each session homework-assignments are given, such as keeping a 'mood diary'. Participants were advised to complete one session per week. No professional assistance was offered.

Participants in the CYL\&TAU group were also advised to visit their GP for treatment of their depressive complaints. GPs were advised to follow the depression guideline as described by the Dutch College of General Practitioners (van Marwijk et al., 2003).

\section{Analyses}

All available data were used for the analyses. Only intermittent missing data were imputed (for 3 participants an intermittent time-point was missing); we calculated the mean of the values of a previous and a subsequent time point. All other missing data (i.e. missing values due to lost to follow-up) were not replaced by imputed values. We tested all effects at the $p<.05$ level (two-tailed). Preliminary tests for distribution and outliers were performed. Skewness and Kurtosis analyses indicated deviations from normality for the track and trace variables, the CEQ, and for the difference scores on the DAS-A-17. The current study includes a posteriori exploratory analyses. However, the power calculations were based on a priori elementary head-to-head comparisons of the three interventions. All analyses were carried out using SPSS (version 15.0.1 for Windows).

First, we described which aspects of CYL patients have used and how acceptable the intervention was, stratified according to treatment type. Frequency differences in categorical variables between the two treatment groups were tested using Chi-square tests. To explore mean differences in continuous variables independent samples t-tests were used. Non-parametric Mann-Whitney tests were used for the CEQ subscales and the track-and-trace variables.

Second, backward logistic regression analysis was used to investigate which variables (i.e. demographic factors, depressive severity, and acceptability factors) predicted the uptake of CYL, which was defined as having completed at least one session (yes $=1 ;$ no $=0$ ). All variables were first tested in univariate models. Promising predictors $(p<.10)$ were entered in a multivariate model controlling for treatment type. Using backward elimination, only variables that were significantly associated with the outcome $(p<.05)$ were retained in the final model. All variables were dichotomised prior to the analyses in order to be more conservative and to be able to interpret the OR's. Continuous variables were dichotomised based on the median value. Education was categorised as follows: low/medium to high.

Third, use and acceptability factors were associated with depressive improvement for the short-term (3-months) and the long-term (9-months) separately. Improvement was defined as a decrease of at least 9 points on the BDI-II (yes $=1$; no $=0$ ), 
based on the reliable change index (Jacobson \& Truax, 1991). A series of simultaneous stepwise logistic regression analyses was conducted, each involving entering the variable of interest in the first step, controlling for potential confounders (i.e. gender, age, education, baseline BDI-II score, and treatment type) in the second step. For the short-term, associations between use of CYL and improvement are merely correlational, i.e. improvement can result in more or less use of CYL or vice versa. Therefore, to analyse the long-term outcome we additionally adjusted for short-term depressive improvement in third step, defined as reliable change between 0 and 3 months. This way, we could determine the unique contribution of the use of CYL to long-term improvement when short-term improvement is partialled out.

Finally, we used the same procedure to test whether use and acceptability of CYL could predict changes on the DAS-A-17, both for the short-term (3-months) and the long-term (9-months) separately. The DAS-A-17 change scores were dichotomised based on the median (high change $=1$; low change $=0$ ). 


\section{Results}

\section{Study participants}

Two-hundred depressed patients were randomly allocated to CYL $(N=100)$ or CYL\&TAU $(N=100)$. At three months of follow-up, data were available for 190 participants (attrition rate 5\%) and at nine months for 178 participants (attrition rate 11\%). Baseline characteristics of the participants are shown in table 1, stratified according to treatment type. As can be seen, initial depressive severity was high and many had a current diagnosis of major depression (CYL: $N=79$; CYL\&TAU: $N=83$ ).

\section{Pre-treatment acceptability}

Scores for expectancy and credibility on the CEQ were moderately high (see table 1). Furthermore, the majority of patients expected that they would be less depressed after treatment or expected that they would learn to cope with their depression. Only few expected that the treatment would cure their depression or would have no effect at all. Most patients were satisfied with the treatment allocation. There were no statistically significant differences between the two treatment groups (all $p>.05$ ).

\begin{tabular}{|c|c|c|}
\hline Variable & CYL $(N=100)$ & CYL\&TAU $(N=100)$ \\
\hline Male sex, $\mathrm{nr}(\%)$ & $48(48.0)$ & $37(37.0)$ \\
\hline Age, $M(S D)$ & $44.3(11.8)$ & $45.2(10.9)$ \\
\hline \multicolumn{3}{|l|}{ Education, $\mathrm{nr}(\%)^{\mathrm{a}}$ : } \\
\hline - Low & $18(18.6)$ & 17 (17.5) \\
\hline - Medium & $55(56.7)$ & $52(53.6)$ \\
\hline - High & $24(24.7)$ & $28(28.9)$ \\
\hline Partner, yes, nr (\%) & $72(73.5)$ & $71(73.2)$ \\
\hline Employed, yes, $\mathrm{nr}(\%)^{\mathrm{c}}$ & $67(72.0)$ & 69 (73.4) \\
\hline Depressive severity (BDI-II), $M(S D)$ & $28.2(7.7)$ & $27.4(8.2)$ \\
\hline Dysfunctional beliefs (DAS-A-17), $M(S D)$ & $72.8(20.4)$ & $71.1(19.8)$ \\
\hline Major depressive episode, $\mathrm{nr}(\%)$ & $79(79.0)$ & $83(83.0)$ \\
\hline Expectancy (CEQ), $M(S D)$ & $18.3(4.2)$ & $19.0(4.8)$ \\
\hline Credibility (CEQ), $M(S D)$ & $18.8(4.0)$ & $19.2(3.8)$ \\
\hline \multicolumn{3}{|l|}{ Expectancy, $\mathrm{nr}(\%)$ : } \\
\hline - The treatment will cure my depression & $13(13.0)$ & $12(12.0)$ \\
\hline - After the treatment I will be less depressed & $33(33.0)$ & $33(33.0)$ \\
\hline - I will learn how to cope with my depression & $50(50.0)$ & $44(44.0)$ \\
\hline - I will learn to accept my depression & $2(2.0)$ & $6(6.0)$ \\
\hline - The treatment will not affect my depression & $2(2.0)$ & $5(5.0)$ \\
\hline \multicolumn{3}{|l|}{ Satisfaction with allocation, $\mathrm{nr}(\%)$ : } \\
\hline - Yes & $66(66.0)$ & $71(71.0)$ \\
\hline - Neutral & $29(29.0)$ & $25(25.0)$ \\
\hline - No & $5(5.0)$ & $4(4.0)$ \\
\hline \multicolumn{3}{|c|}{$\begin{array}{l}\text { Note. } \mathrm{CYL}=\text { Colour Your Life (unsupported online CCBT); TAU = treatment as usual; BDI-II = } \\
\text { Beck Depression Inventory II (score range 0-63); DAS-A-17 = 17-item Dysfunctional Attitude } \\
\text { Scale (score range 17-119); CEQ = Credibility/expectancy Questionnaire (score range 3-27). } \\
\text { a data unavailable: CYL }(N=3) ; \mathrm{CYL \& TAU}(N=3) ;{ }^{\mathrm{b}} \text { data unavailable: CYL }(N=2) ; \text { CYL\&TAU } \\
(N=3) .{ }^{\circ} \text { data unavailable: CYL }(N=7) ; \text { CYL\&TAU }(N=6) \text {. }\end{array}$} \\
\hline
\end{tabular}




\section{Use of the CYL intervention}

Table 2 depicts the use of CYL stratified according to treatment type. The majority of patients completed the first session (CYL: $N=72$; CYL\&TAU: $N=76$ ), while very few completed all sessions (CYL: $N=2$; CYL\&TAU: $N=7$ ). The mood diary site and homework site were used very often. Nearly half of all homework assignments were made and filled out on the website. There are no major differences between the two treatment groups, except for the completion of the eighth session, which was completed by significantly more patients in the CYL\&TAU group $(N=26)$ than in the CYL only group $(N=14)(p<.05)$.

Table 2 Usage of the CYL program

\begin{tabular}{|c|c|c|}
\hline Variable & CYL $(N=100)$ & CYL\&TAU $(N=100)$ \\
\hline Session 1 completed, $N$ & 72 & 76 \\
\hline Session 2 completed, $N$ & 63 & 60 \\
\hline Session 3 completed, $N$ & 52 & 55 \\
\hline Session 4 completed, $N$ & 42 & 50 \\
\hline Session 5 completed, $N$ & 36 & 47 \\
\hline Session 6 completed, $N$ & 31 & 39 \\
\hline Session 7 completed, $N$ & 25 & 36 \\
\hline Session 8 completed, $N$ & 14 & $26^{\star}$ \\
\hline Booster session completed, $N$ & 2 & 7 \\
\hline Sessions completed, $M(S D)$ & $3.4(3.0)$ & $4.0(3.4)$ \\
\hline Homework assignments completed, $M(S D)^{\mathrm{a}}$ & $5.4(5.0)$ & $6.2(5.3)$ \\
\hline Mood diaries completed, $M(S D)^{\mathrm{b}, \mathrm{c}}$ & $58.1(86.4)$ & $53.9(75.5)$ \\
\hline Number of logins, $M(S D)^{c}$ & $26.1(53.9)$ & $22.7(29.6)$ \\
\hline Total time spent, in minutes, $M(S D)$ & 227 (249) & $233(218)$ \\
\hline Time spent per login, in minutes, $M(S D)$ & $11(10)$ & $11(9)$ \\
\hline \multicolumn{3}{|l|}{ Number of visits, $M(S D)^{c}$ : } \\
\hline - Homework site & $62.1(98.6)$ & $67.8(88.0)$ \\
\hline - Mood diary site & $191.2(296.7)$ & $179.7(222.0)$ \\
\hline - Forum & $4.1(11.1)$ & $3.6(7.6)$ \\
\hline
\end{tabular}

Note. $\mathrm{CYL}=$ Colour Your Life (online, unsupported CCBT); TAU = treatment as usual.

a 13 assignments could be completed; ${ }^{\mathrm{b}}$ Mood diary could be filled out daily; ${ }^{\circ}$ Data unavailable: CYL $(N=2)$; CYL\&TAU $(N=1)$.

${ }^{*} p<.05$ (chi-square test). 


\section{Predicting uptake of CYL}

As can be seen in table 3, only gender and education significantly predicted the uptake of $C Y L$ in both the univariate and multivariate analyses. Men had a significantly lower odds than women $(O R=0.39$ in the multivariate model) to take up CYL. A significantly higher odds was found for being highly educated compared with medium to low education $(O R=3.12$ in the multivariate model) for uptake of $C Y L$.

\begin{tabular}{lll}
\multicolumn{3}{l}{ Table 3 Predicting uptake of CYL: results from backward logistic regression analysis } \\
\hline Variable & $\begin{array}{l}\text { Univariate } \\
\text { OR }(95 \% \mathrm{Cl})\end{array}$ & $\begin{array}{l}\text { Multivariate } \\
\text { OR }(95 \% \mathrm{Cl})\end{array}$ \\
\hline Predictors: & & \\
Gender (male) & $0.39(0.20-0.74)^{\star \star}$ & $0.39(0.20-0.77)^{\star \star}$ \\
Age (>46 years) & $0.77(0.41-1.44)$ & \\
Education (high) & $2.98(1.39-6.40)^{\star \star}$ & $3.12(1.42-6.87)^{\star \star}$ \\
Partner (yes) & $0.72(0.33-1.53)$ & \\
Employed (yes) & $1.35(0.66-2.79)$ \\
Baseline BDI-II score (>26) & $1.30(0.69-2.45)$ & \\
Major depressive episode (yes) & $1.90(0.90-4.04)^{\#}$ & \\
CEQ - credibility (>19) & $1.08(0.57-2.03)$ & \\
CEQ - expectancy $(>19)$ & $0.99(0.53-1.87)$ & \\
Satisfaction (yes) & $1.71(0.88-3.31)$ & \\
Covariate: & & $1.16(0.59-2.27)$ \\
Treatment (CYL\&TAU) & $1.23(0.65-2.32)$ &
\end{tabular}

Note. $\mathrm{CYL}=$ Colour Your Life (unsupported online CCBT); TAU = treatment as usual; BDI-II = Beck Depression Inventory II; CEQ = Credibility/expectancy Questionnaire. Uptake of CYL was defined as having completed at least one session of CYL (yes: $N=148$; no: $N=52$ ). For all variables: the indicator category is in brackets and the reference category is the contrast. Univariate associates with a $p$-value $<.10$ were included in the multivariate model. In the multivariate model only significant predictors $(p<.05)$ and covariates are shown after backward elimination.

${ }^{\#} p<.10 ;{ }^{\star *} p<.01$.

\section{Factors related to depressive improvement}

Results of the logistic regression analysis testing associations of use and acceptability factors with depressive improvement are summarised in table 4. At three months, homework assignments, number of logins, time spent, and visits to mood diary were significantly and positively associated with improvement at three months (all $p<.05$ ). When controlling for potential confounders, only number of logins, time spent and visits to mood diary remained significantly associated with the outcome.

At nine months, expectancy and all track and trace variables showed significant associations with depressive improvement (all $p<.05$ ) in the first and second steps. However, when we added short-term depressive improvement to the model in a third step, to single out improvement attributable to the preceding use of CYL, only expectancy $(O R=2.21)$ and homework $(O R=2.34)$ remained significantly and strongly associated with depressive improvement. 


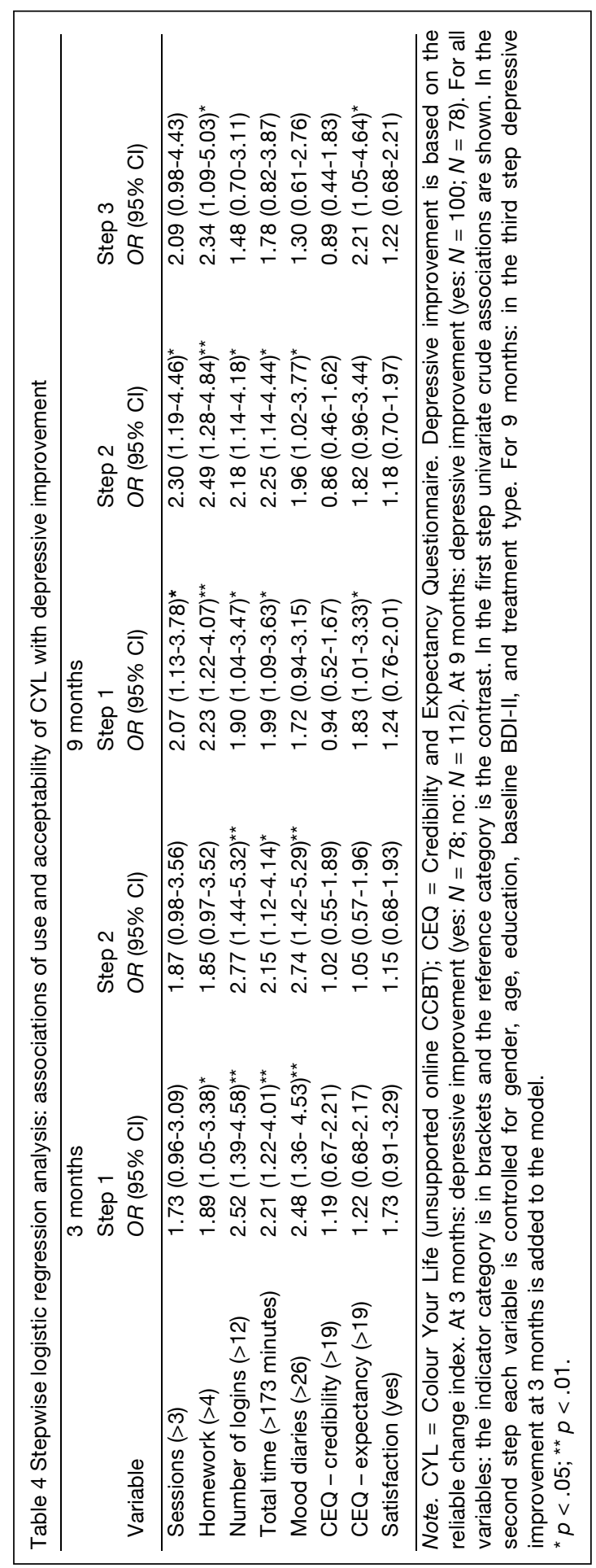




\section{Factors related to change in dysfunctional attitudes}

Table 5 depicts the results from the regression analyses examining factors that are associated with change in dysfunctional attitudes. Another pattern of results was found compared to the analysis with depressive improvement as dependent variable. At three months, only number of logins was positively associated with the outcome in the crude and adjusted model (both $p<.05$ ), while at nine months only expectancy was significantly and positively related to change in dysfunctional attitudes, even when controlling for potential confounders $(O R=2.85)$.

\section{Treatment evaluation by patients}

In table 6, patients' post-treatment evaluation of the intervention is shown, stratified according to treatment type, protocol compliance, and depressive improvement. In general, patients were relatively neutral in their evaluation. Patients in the CYL\&TAU group rated the treatment as less impersonal than in the CYL only group $(p<.01)$. Furthermore, improved patients were significantly more positive about the intervention than patients who did not improve. 


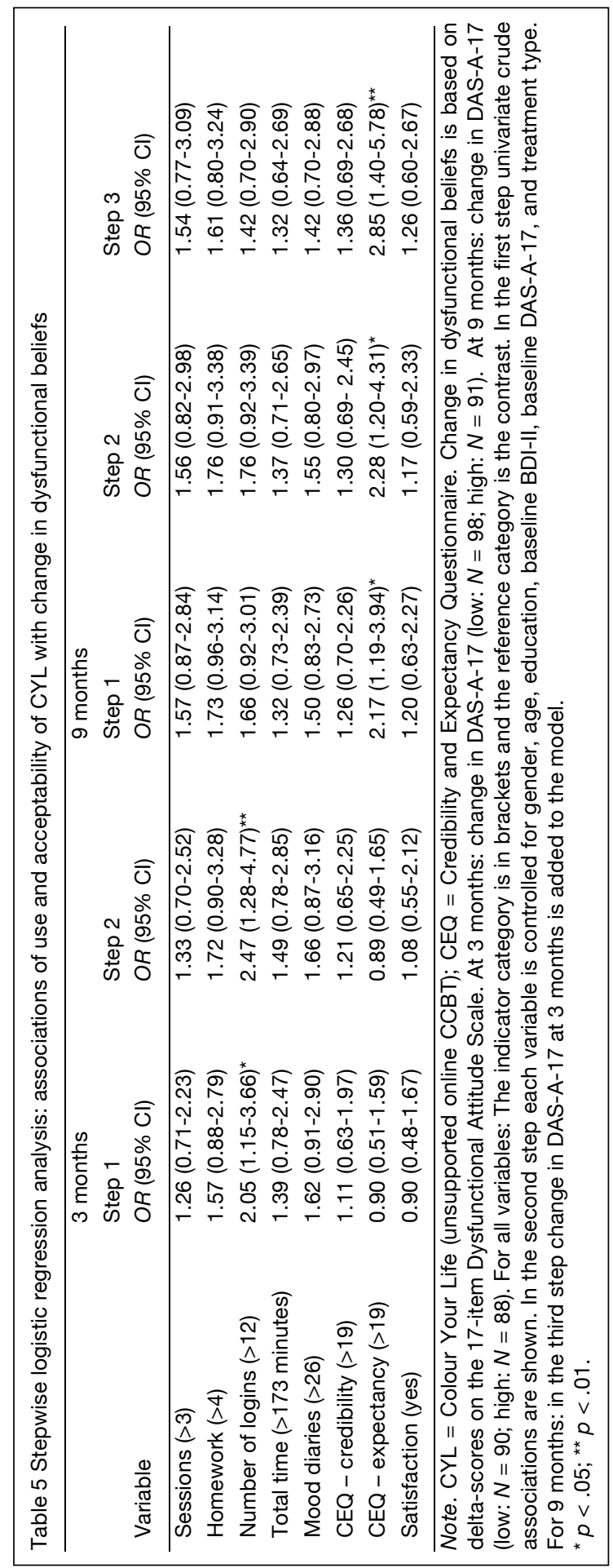




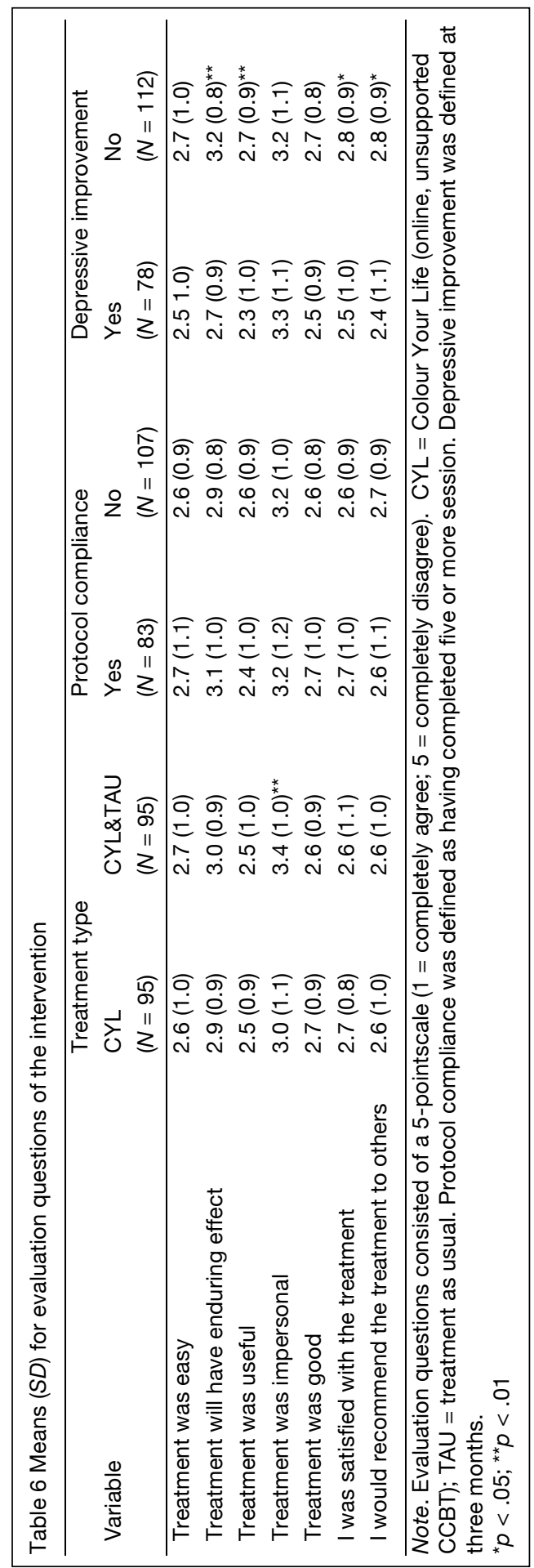




\section{Discussion}

\section{Main findings}

The aim of the current study was to determine how use and acceptability of online, unsupported CCBT (i.e. Colour Your Life/CYL) were related to the uptake of the intervention and to outcome in terms of depressive improvement and change in dysfunctional attitudes. Patients had rather positive expectations before the start of the intervention, whereas after the intervention patients were somewhat neutral in their ratings. Patients who improved were more positive about the intervention. Furthermore, many patients started with the intervention, but few completed all sessions. Gender and education - not depressive severity or pre-treatment expectations - predicted uptake of CYL. Many CYL usage indices, but not the acceptability factors, were associated with short-term improvement of depression. For the long-term, only homework and expectancy were significantly associated with depressive improvement, after short-term improvement had been partialled out. Regarding change in dysfunctional attitudes, only number of logins was associated with short-term outcome, whereas expectancy was the only factor that was related to the long-term outcome.

\section{Usage of CCBT}

In the current study, the uptake of CYL was rather good; most patients (i.e. 74\%) started with CYL and completed their first session. This seems comparable to previous studies (e.g. Proudfoot et al., 2003; Spek et al., 2007b), although lower uptake rates have also been found (see Kaltenthaler et al., 2008b). Perhaps the formal structure of a randomised trial might account for larger take-up rates (see also Christensen et al., 2004b).

Although uptake appears good, many patients (i.e. $86 \%$ in the CYL group and $74 \%$ in the CYL\&TAU group) dropped out at some point during the intervention. Mental health treatment dropout in general is a serious problem (Edlund et al., 2002), but the problem seems even bigger in CCBT (Kaltenthaler et al., 2008a; Kaltenthaler et al., 2008b). High drop-out rates seem to be more prevalent in Internet-delivered CCBT than in CCBT delivered in other ways (e.g. see Christensen et al., 2006b; Kaltenthaler et al., 2008b), especially when offered without any support (Clarke et al., 2002; Patten, 2003; Spek et al., 2007a). Despite our high dropout rate, half of the patients completed four to five sessions. So patients might have been exposed to at least some essential steps of the intervention. However, many short visits (i.e. 11 minutes) were made to the program. This might indicate that patients had difficulties to concentrate and complete a session in one visit. Perhaps the intervention was too demanding for our sample of moderately to severely depressed patients. These visits might have been too short to actually recover from depression. This might explain the lack of effectiveness of CYL, as we showed in our previous study (de Graaf et al., in press-a).

With regard to predictors of the uptake of CYL, we found that women and highly educated patients were most likely to complete the first session, which is consistent with previous research (e.g. see Jorm et al., 2004; McKendree-Smith et al., 2003). Although for more seriously depressed individuals it might be more difficult to motivate oneself to start using self-help materials, initial depressive severity was not predictive of CYL uptake. However, our sample had a limited variation in depressive severity. Furthermore, pre-treatment acceptability did not predict CCBT uptake either as was suggested in previous studies (Marks et al., 1998b; Murray et al., 2003). Differences in 
study designs, populations, and treatment types might account for this.

In clinical practice, these findings indicate that improving adherence to CCBT is needed. Telephone calls, postcard reminders and short face-to-face contact have effectively increased treatment adherence for several psychological disorders (Andersson et al., 2005; Carlbring et al., 2007b; Christensen et al., 2004a; Clarke et al., 2005; Farvolden et al., 2005; Kenwright et al., 2005). Furthermore, our findings suggest that different methods could be used for different groups of patients. For example, highly educated patients might be more capable of using pure self-help, while patients with lower levels of education might be better of with some additional support. Clearly more research regarding this topic is needed.

\section{Acceptability of CCBT}

Before treatment, patients rated CYL as an acceptable treatment, in terms of expectancy and credibility. In previous studies similar pre-treatment expectations were reported, but only after a demonstration of CCBT that was intended to remove any misconceptions (Mitchell \& Dunn, 2007; Mitchell \& Gordon, 2007). In our study, we only provided some practical information, suggesting that an extensive demonstration might not be needed. We must bear in mind here that our participants were all willing to use CCBT. Consequently, they might differ in their expectations from those who did not to take part in our study.

Regarding post-treatment acceptability of CYL, patients were relatively neutral (i.e. they did not agree or disagree with the evaluation statements), whereas previous studies reported more positive outcomes (Kaltenthaler et al., 2008b; Marks et al., 1998b; Osgood-Hynes et al., 1998). However, these studies have only reported data for treatment completers but not for dropouts, which might have elevated their judgment about the acceptability. Although we were able to provide information for all participants irrespective of compliance, compliers and dropouts had similar acceptability ratings. We did find differences in acceptability ratings regarding clinical improvement; patients whose depressive symptoms improved were more positive. This might be explained by attributing reduction in depressive symptoms to CYL.

Furthermore, we showed that pre-treatment expectancy, but not credibility, was positively related to long-term reduction in depressive symptoms and dysfunctional attitudes, which is in line with other research (see Goossens et al., 2005). Surprisingly, we were not able to show that expectancy and credibility were associated with shortterm treatment outcome. We can only speculate about the meaning of this. Perhaps an underlying patient characteristic, which becomes only visible when the depressive symptoms have been lifted, accounts both for positive expectations and clinical improvement. More research, especially qualitative evaluation of good methodological quality about the acceptability of CCBT might shed more light on this topic (see also Kaltenthaler et al., 2008b; Waller \& Gilbody, in press). Such studies might more sufficiently describe why participants withdrew from CCBT than quantitative data.

\section{Factors associated with clinical outcome}

Consistent with previous research (e.g. Christensen et al., 2006a; Manwaring et al., 2008), we found that when the program was used more frequently, the odds were much larger to show short-term depressive improvement even when controlling for known predictors, such as initial depressive severity (Hamilton \& Dobson, 2002; Spek 
et al., 2008b). This seems to suggest that adherence to CCBT is important to reduce depressive symptoms. However, the dose-response relation we found, might be due to the retention of patients who showed the biggest improvements early (Christensen et al., 2006a), or due to increased despondency in those who were not capable of using the self-help intervention (Williams \& Whitfield, 2001). The fact that the active ingredients of CYL, that is sessions and homework assignments, were not related to short-term improvement seems to support this notion. Previously, it has been suggested that symptomatic improvement in traditional CBT is initially influenced by nonspecific factors and occurs prior to the formal introduction of active ingredients of CBT (llardi \& Craighead, 1994).

Regarding the relation between usage of CYL and long-term outcome, only homework was significantly associated with depressive improvement. This suggests that it might take some time before the patient has incorporated the cognitive and behavioural techniques. In traditional CBT compliance with homework assignments can positively contribute to treatment outcome (e.g. Addis \& Jacobson, 2000; Kazantzis et al., 2000). Especially in unsupported computerised self-help, homework might be an important ingredient. Because we controlled for early change in depression here, we feel more confident that this finding indicates a causal role for homework compliance in determining long-term depressive improvement. However, we cannot rule out the possibility that a third variable is responsible for both homework compliance and outcome (see also Addis \& Jacobson, 2000). Moreover, adding short-term depressive improvement to the model could have partialled out true effects of usage of CYL on long-term change in depression. We should note that the track-and-trace system only registered whether homework was filled out online, but some might have filled out nonsense and others might have printed the assignments and did them on paper.

A central theoretical principle of cognitive therapy is that changes in automatic thoughts and underlying schema's are responsible for reduction in depressive symptoms (Beck et al., 1979). We therefore investigated the effects of CYL usage on the reduction of dysfunctional beliefs. We should note that any association might only indicate that dysfunctional beliefs covary with depressive severity, which was previously found by Selmi et al. (1990) for CCBT. To our surprise, none of the usage indices were associated with changes in dysfunctional attitudes, except for number of logins. This seems counterintuitive, since CBT involves challenging depressogenic cognitions (Beck et al., 1979). Previously, it was suggested that the importance of the content of dysfunctional beliefs in depression should be reconsidered (Beevers et al., 2003; Teasdale et al., 2001). However, since we are among the first to relate usage of CCBT to dysfunctional beliefs, we need to be careful in interpreting this finding.

In general, our findings suggest that adherence to CYL only covaries with reduction in depression and dysfunctional beliefs. However, the correlational nature of our study does not allow cause-effect conclusions; experimental designs are needed. Christensen et al. (2006b), for instance, compared the effects of brief and full online CCBT. Although the full package was more effective, it was associated with a higher dropout rate. Clearly, more research is needed to define the optimum balance between length of the intervention, effectiveness, and therapy drop-out. 


\section{Methodological considerations}

The current study has several strengths. We had a relatively low attrition rate regarding data collection, so we feel confident that no biases occurred due to missing data. Furthermore, we were able to recruit patients from the general population. Unlike in samples selected in general practices or clinics, no biases occurred due to help seeking behaviour of patients and illness recognition by physicians, which is often a problem in depression (Paykel et al., 1997). However, we should note that our sample was perhaps too depressed in relation to the scope of the intervention. Our sample was more severely depressed than in previous studies (Andersson et al., 2005; Proudfoot et al., 2004) and the mean starting levels on the BDI-Il were even higher than generally seen in primary care patients with major depression (Vuorilehto et al., 2007). This might limit the generalizability of our findings. Moreover, it is uncertain whether treatment adherers are representative of the depressed population. Some other limitations of the present study should also be noted. Firstly, as mentioned earlier, our study was not designed to disentangle dose-response relationships and the findings are purely correlational. Secondly, all our outcomes were measured online and one might question the equality of computerised questionnaires and paper-and-pen versions. However, there are sufficient indications that computerised and paper-and-pen questionnaires show similar psychometric properties (Carlbring et al., 2007a; Garb, 2007; Schulenberg \& Yutrzenka, 2001). Thirdly, for the regression analyses we dichotomised all variables based on the median because of non-normality and to be more conservative. However, using the median might be an arbitrary choice and other cut-off points might have resulted in other outcomes. Fourthly, we relied on self-report measures at follow-up and, as a result, we have no information on actual diagnoses of depressive episodes at follow-up. Fifthly, the statistical analyses were performed a posteriori and power analyses were lacking for these tests. Together with the absence of clear hypotheses, this might have increased the chance of type I error. Finally, we should note that the track and trace variables are mainly a proxy of the use of the intervention. Due to privacy reasons, we were not able to check for instance the content of the homework assignments.

\section{Conclusion}

The good pre-treatment acceptability and high uptake rate are indications for the feasibility of unsupported online CCBT for depression. However, many patients dropped out of treatment and means to improve treatment adherence might thus be indicated. Furthermore, in our exploratory analyses we could only find few indications that usage of CYL was related to treatment outcome. Perhaps CCBT, offered online without any support, is not beneficial for moderately to severely depressed patients. Adding therapist support to online self-help interventions might improve adherence. Compliance with homework assignments should also expressly be encouraged and positive treatment expectancy in CCBT should be facilitated. 


\section{Acknowledgements}

We thank Annie Hendriks and Greet Kellens for their assistance during the study and Rosanne Janssen for the development of the infrastructure for online data-collection. The trial is financed by ZonMw (Netherlands Organisation for Health Research and Development; project number 945-04-417), research institute EPP and research institute CAPHRI. Municipalities Eijsden, Meerssen, Sittard-Geleen, Valkenburg and Maastricht sponsored the study. 


\section{Chapter 9}

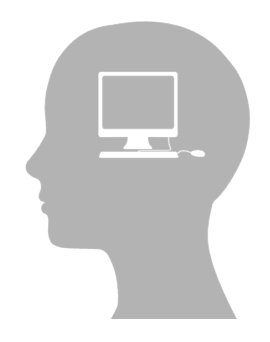

\section{Predicting outcome in computerised cognitive}

behavioural therapy for depression in primary care: a randomised trial

L.E. de Graaf, S.D. Hollon \& M.J.H. Huibers 


\section{Abstract}

Objective: To explore pre-treatment and short-term improvement variables as potential moderators and predictors of 12-month follow-up outcome of unsupported online CCBT and CCBT combined with usual care for depression.

Methods: 303 depressed patients were randomly allocated to (a) unsupported online CCBT (b) treatment as usual (TAU), or (c) CCBT and TAU combined. Potential predictors/moderators were demographic, clinical, cognitive, and short-term improvement variables. Outcomes were the BDI-II score at 12 months of follow-up and reliable change.

Key findings: Those with higher levels of extreme (positive) responding had a better outcome in CCBT compared to TAU, while those having a parental psychiatric history or a 'major depressive disorder' diagnosis had a better outcome in CCBT\&TAU compared to TAU. Predictors regardless of treatment type included current employment, low pre-treatment illness severity, and short-term improvement on clinical variables. Conclusions: CCBT might tap into latent emotional tendencies that stimulate improvement, whereas CCBT\&TAU might be the most suitable option for those with more severe vulnerability characteristics. Those with the least impairment improve the most, regardless of treatment type. 


\section{Introduction}

Depression is a common complaint in the general population (Bijl et al., 1998). Although depression can be treated effectively with cognitive behavioural therapy (CBT) (e.g. see Butler et al., 2006; Hollon et al., 2006), many depressed individuals remain untreated (Hirschfeld et al., 1997) due to high cost and therapists' shortage (Bower et al., 2003). Computerised CBT (CCBT) might be a promising solution to this problem. CCBT can be offered as a self-help intervention with or without support.

There is ample evidence supporting the efficacy of CCBT for depression. In a recent systematic review, Kaltenthaler and colleagues (2006) found that CCBT appeared as effective as face-to-face CBT and more effective than usual care. However, this conclusion was mainly based on research that was conducted outside the reach of primary care. Proudfoot et al. (2004) specifically investigated the efficacy of CCBT for depression in primary care, and it was shown that supported CCBT - delivered on a personal computer in the general practice - is more effective than usual care by a general practitioner (GP) in mild to moderate depression and anxiety. Recently, we conducted a randomised trial in which we evaluated the effectiveness of unsupported online CCBT (i.e. Colour Your Life) for depression in primary care compared with treatment as usual by a GP and a combination of both treatments (de Graaf et al., in press-a). In our study, CCBT did not outperform usual GP care and CCBT in conjunction with usual care had no additional effects. Moreover, the overall effects of the interventions in this group of moderately to severely depressed patients were modest.

To gain more insight into the differential outcomes in this group of patients, we aimed to identify predictors and moderators of treatment for depression status during one-year of follow-up. Predictors, or prognostic indicators, are baseline variables that predict response irrespective of treatment. Moderators, or prescriptive indicators, are baseline characteristics that interact with treatment. They inform us which treatment is most suitable for whom (Kraemer et al., 2002). Such knowledge could help facilitating optimal treatment selection, improving depression care, and preventing poor treatment response. Few previous CCBT studies have conducted such analyses.

Several baseline factors have been linked to poor outcome in depression across various treatments (i.e. both psychotherapy and pharmacotherapy), such as high pre-treatment illness severity (Jarrett et al., 1991), chronic and recurrent depression (Hamilton \& Dobson, 2002; Persons et al., 1988; Thase et al., 1994), and being single (Jarrett et al., 1991). Predictors for depressed primary care patients are mostly similar and also include: lower levels of education, unemployment, older age, male gender, decreased social functioning, and the presence of personality disorders and co-morbid axis I disorders (for an overview see Wolf \& Hopko, 2008). Recently, Petersen et al. (2007) showed that an extreme response style significantly predicted poor treatment outcome in recurrent and chronic depression. Only few studies have been able to show patient characteristics that are specifically related to outcome in CBT. For instance, those with high pre-treatment levels of dysfunctional attitudes seem to do better in CBT than in pharmacotherapy (Hamilton \& Dobson, 2002; Jarrett et al., 1991). In contrast, depressed patients with a comorbid personality disorder were more likely to improve using antidepressant medication than in cognitive therapy in the short-term. Nevertheless, those with personality disorders that did respond to cognitive therapy stayed that way (Fournier et al., 2008). 
Despite a considerable number of these prognostic studies, few data are available on predictors and moderators of outcome in CCBT. It cannot merely be assumed that the same patient characteristics will be as relevant in CCBT as in other interventions. We know of only two studies that specifically focused on predictors of CCBT outcome in depression. Similar to other treatments, higher self-reported depressive severity, poorer quality of life, and higher number of previous episodes were associated with poorer outcome in patients that had received online CCBT with minimal therapist contact (Andersson et al., 2004). Recently, Spek et al. (2008b) reported predictors and moderators for CCBT and group CBT in people over 50-years old with subthreshold depression. Higher educational level, female gender, higher baseline depressive severity, and higher neuroticism scores predicted greater reduction in depressive symptom, irrespective of treatment. Furthermore, those with high scores on the personality characteristic 'altruism' did better in group CBT than in CCBT (Spek et al., 2008b).

Several studies have examined whether initial improvement is predictive of longer-term outcome in depression. To our knowledge, no such studies have been conducted in relation to CCBT. During drug treatment, the most powerful predictor of outcome is initial treatment response (Mulder et al., 2006), although in another study this was not found for initial improvement during a placebo lead-in period (Marie-Mitchell et al., 2004). In primary care, initial improvement was predictive of outcome in neurotic disorders five years later (Seivewright et al., 1998). Similar findings were obtained for adolescent psychosocial treatment (Renaud et al., 1998) and for combined treatment (Van et al., 2008). However, none of these studies have found that initial improvement was a specific predictor for a certain treatment.

The present study will examine moderators and predictors of treatment outcome using data from our recent randomised trial (de Graaf et al., in press-a). Based on previous literature, we will focus on demographic variables (e.g. gender, age, and education), clinical factors (e.g. illness severity, co-morbid anxiety disorder, and quality of life), cognitive factors (i.e. dysfunctional attitudes and extreme responding), and short-term improvement variables (i.e. improvement in clinical and cognitive variables during the first three months). 


\section{Method}

\section{Design}

The design of the study was a randomised trial with three conditions: (a) unsupported online CCBT, (b) TAU by a GP, and (c) CCBT and TAU combined. The Medical and Ethical Committee approved the study protocol. The study is registered at the Netherlands Trial Register, part of the Dutch Cochrane Centre (ISRCTN47481236). Details of the study method have been described elsewhere (de Graaf et al., 2008a).

\section{Study population}

Participants $(N=303)$ were recruited in the general population by means of a largescale Internet-based screening in the South of the Netherlands. A random selection of individuals was sent a postal invitation to participate in the screening. Potentially eligible participants were invited to visit the research centre for an intake to assess final eligibility. Participants were eligible if they met the following criteria: age 18 to 65 ; access to the Internet at home; at least mild to moderate depressive complaints (BDI-II score $\geq 16$ ); duration of depressive complaints 3 months or more; no current psychological treatment for depression; no continuous antidepressant treatment for at least 3 months prior to entry; fluent in Dutch language; no alcohol and/or drug dependence; and no severe psychiatric co-morbidity.

\section{Procedure}

After written informed consent was obtained, participants were randomly allocated to one of the three interventions. Baseline assessment (including a diagnostic interview and questionnaires) took place at the research centre before randomisation. The questionnaires were administered on a computer. All follow-up assessments took place at home via the Internet at two, three, six, nine, and twelve months after inclusion. Preceding an upcoming assessment point, participants received an e-mail alert. If a participant failed to complete the assessment within one week, an e-mail reminder was sent. When the participant still failed to fill out the questionnaires, they were encouraged to respond by phone.

\section{Interventions}

The CCBT program, named Colour Your Life (CYL), is an online, multimedia, interactive, self-help computer program for depression based on the principles of CBT (Riper \& Kramer, 2004). The program consists of eight weekly sessions, a ninth booster session, and thirteen homework assignments. No assistance was offered.

TAU was delivered by the patient's own GP who was advised to follow Dutch depression guidelines (van Marwijk et al., 2003). TAU can include four to five biweekly consultations and antidepressant treatment if indicated.

\section{Outcomes}

\section{Demographics}

The following demographic variables were assessed during the online screening process: age, gender, marital status, education, and employment status. 


\section{Composite International Diagnostic Interview}

To determine current DSM-III-R axis I diagnoses, the computerised Composite International Diagnostic Interview (CIDI-auto) was used, which has acceptable reliability and validity (Robins et al., 1988; Wittchen, 1994). The following variables were used in the current study: major depressive episode, co-morbid anxiety, and total number of axis I disorders. The CIDI was administered only at baseline.

\section{Beck Depression Inventory II}

The Beck Depression Inventory Second Edition (BDI-II) measures the severity of depression with high scores indicating severe depression (range 0-63). There has been consistent support for its construct validity and reliability in various samples (Beck et al., 1996; van der Does, 2002).

\section{Symptom Check-List 90}

General psychopathology (i.e. total level of emotional dysfunction) was measured with the Symptom Check-List 90 (SCL-90), with a score range from 90 (no psychopathology) to 450 (severe psychopathology). The SCL-90 has a high degree of reliability and support has been found for its validity (Arrindell \& Ettema, 1981; Derogatis et al., 1976).

\section{6-item short-form Health Survey}

The 36-item short-form Health Survey (SF36) was used to assess specific features of quality of life. We used the two most relevant subscales: role limitations caused by emotional problems and general mental health. High scores indicate low levels of role limitations and high levels of general mental health (score range 0-100). The SF36 has good psychometric properties in terms of validity, reliability, and scale structure (Aaronson et al., 1998; McHorney et al., 1993; Ware \& Sherbourne, 1992).

\section{Dysfunctional Attitude Scale form A}

The intensity of dysfunctional beliefs was assessed with the Dysfunctional Attitude Scale form A (DAS-A) (Weissman \& Beck, 1978). We used a 17-item version, which demonstrated good reliability $(a=.91)$ and convergent construct validity (de Graaf et al., in press-b). We used two subscales: 'perfectionism/performance evaluation' (11 items; score range 11-77) and 'dependency' (6 items; score range 6-42). Higher scores indicate more dysfunctional attitudes. In addition, extreme positive and extreme negative responding was investigated by calculating the number of extreme responses (i.e. 'fully agree' and 'fully disagree' respectively) on the 17-item DAS-A (see also Petersen et al., 2007; Teasdale et al., 2001).

\section{Questionnaire without Questions}

Because extreme responding to depression related questionnaires, such as the DASA-17, might be confounded by depressive severity, extreme responding was also explored using the Questionnaire without Questions (QwQ) (Berg \& Rapaport, 1954; de Graaf et al., 2008b). Individuals have to choose between various answers, solely based on the presentation of the answers: (1) yes-uncertain-no, (2) very satisfiedsatisfied-unsatisfied-very unsatisfied, and (3) and agree-neutral-disagree. Extreme responding was calculated by adding up the number of extreme responses, negative and positive separately. Test-retest reliability is sufficient (de Jonge \& Slaets, 2005). 


\section{Other candidate variables}

Other potential predictors of depression outcome were: factors related to Internet use in general (i.e. experience and pleasure), parental psychiatric history, parental history of depression, and traumatic childhood experience. All were assessed at baseline by self-report questionnaires, including questions such as "do you enjoy using the Internet?", "has one or both of your biological parents ever been treated by a psychiatrist?", and "when you were a child or teenager, have you ever been physically abused?". Social functioning was measured using the Work and Social Adjustment Scale (WSAS) (Mundt et al., 2002).

\section{Data analysis}

All available data were analysed, regardless of treatment adherence. Only intermittent missing data were imputed $(N=5)$; we calculated the mean of the values of a previous and a subsequent time point. Missing values due to loss to follow-up were not replaced by imputed values. We tested all effects at the $p<.05$ level (two-tailed). Preliminary tests for distribution and outliers indicated no deviations from normality. All analyses were carried out using SPSS (version 15.0.1 for Windows).

To test whether baseline and short-term improvement (i.e. $t_{\text {baseline }}-t_{\text {month } 3}$ ) variables were predictors or moderators, we followed the guidelines of Kraemer et al. (2002). Separate multiple regression models were constructed to evaluate the effect of each variable on the outcome, i.e. BDI-II score at 12 months of follow-up. Each model involved the three variables of interest: (1) treatment type (TAU is the reference category), (2) variable, and (3) treatment type $x$ variable interaction. We controlled for baseline BDI-II score. If there is a significant treatment by variable interaction, with or without main effect, then the variable is a moderator. If there is only a main effect for the variable, then it is a predictor. Next, the relative contribution of significant $(p<.05)$ predictors and moderators were evaluated in a multivariate model after controlling for treatment type and baseline BDI-II using a backward elimination procedure.

In line with recommendations of Steketee and Chambless (1992) to also use a more conservative outcome reflecting change in depression, we additionally investigated potential baseline moderators and predictors of reliable change. This was based on the reliable change index (Jacobson \& Truax, 1991), which is assessed using a variation on the standard error of measurement. Reliable change was defined as a decrease of at least 9 points on the BDI-II (yes $=1$; no $=0$ ). Time to reliable change was estimated using Cox regression analysis, applying the same procedure as in the previous analyses. Patients who were lost at follow-up were treated as censored observations, as were patients who did not reliably change during follow-up (i.e. 2, 3, 6, 9 , and 12 months). $95 \%$ confidence intervals for hazard rations were calculated. It is noted that we did not assess short-term improvement variables as predictors/moderators of reliable change, since change is already reflected in the outcome variable.

Prior to the analyses, continuous variables were transformed into $z$-scores in order to account for scale differences. Results are reported as standard deviation units. We should note that short-term improvement variables could not be established earlier than three months of follow-up, since not all outcomes were measured before that time. 


\section{Results}

\section{Participants}

Three-hundred-and-three depressed participants were enrolled in the study. At 12 months of follow-up, data were available for 267 participants (attrition rate $11.9 \%$ ). The flow of the participants is graphically shown in figure 1 . Table 1 shows the baseline characteristics of all participants stratified according to intervention group.

Table 1 Baseline variables of the total sample $(N=303)$. Data are means $(S D)$ unless stated otherwise.

\begin{tabular}{|c|c|c|c|}
\hline Variable & $\begin{array}{l}\text { CCBT } \\
(N=100)\end{array}$ & $\begin{array}{l}\text { TAU } \\
(N=103)\end{array}$ & $\begin{array}{l}\text { CCBT\&TAU } \\
(N=100)\end{array}$ \\
\hline Male sex, $\mathrm{nr}(\%)$ & $48(48.0)$ & $46(44.7)$ & $37(37.0)$ \\
\hline Age $(18-65)$ & $44.3(11.8)$ & $45.1(12.2)$ & $45.2(10.9)$ \\
\hline Education, low, $\mathrm{nr}(\%)^{\mathrm{a}}$ & $18(18.6)$ & $16(16.2)$ & $17(17.5)$ \\
\hline Employed, yes, $\mathrm{nr}(\%)^{\mathrm{b}}$ & $67(72.0)$ & $64(76.2)$ & $69(73.4)$ \\
\hline Major depressive episode, nr (\%) & 79 (79.0) & $91(88.3)$ & $83(83.0)$ \\
\hline Parental psychiatric history, $\mathrm{nr}(\%)^{\mathrm{C}}$ & $22(23.7)$ & $20(21.1)$ & $21(21.9)$ \\
\hline BDI-II (0-63) & $28.2(7.7)$ & $27.9(7.5)$ & $27.4(8.2)$ \\
\hline SCL-90 (90-450) & $182.9(43.4)$ & $179.9(41.9)$ & $180.0(40.0)$ \\
\hline SF36 - general mental health $(0-100)$ & $44.7(13.7)$ & 44.5 (13.9) & $45.1(14.3)$ \\
\hline SF36 - role limitations $(0-100)$ & $29.7(36.4)$ & $34.0(35.8)$ & $33.7(36.8)$ \\
\hline DAS-A - perfectionism (11 -77) & $36.7(12.0)$ & $36.7(12.0)$ & $36.5(11.9)$ \\
\hline DAS-A - dependency (6 -42) & $26.0(7.0)$ & $25.3(6.5)$ & $25.2(6.5)$ \\
\hline DAS-A - positive responding (0-17) & $1.2(2.3)$ & $1.0(1.8)$ & $1.7(2.9)$ \\
\hline DAS-A - negative responding $(0-17)$ & $0.9(2.0)$ & $0.6(1.4)$ & $0.8(1.6)$ \\
\hline QwQ - positive responding $(0-3)$ & $0.5(0.7)$ & $0.5(0.7)$ & $0.5(0.7)$ \\
\hline QwQ - negative responding $(0-3)$ & $0.3(0.5)$ & $0.3(0.6)$ & $0.3(0.5)$ \\
\hline \multicolumn{4}{|c|}{$\begin{array}{l}\text { Note. CCBT = computerised cognitive behavioural therapy (i.e. CYL offered online without } \\
\text { support); TAU = treatment as usual; BDI-II = Beck Depression Inventory-II; SCL-90 = } \\
\text { Symptom Checklist } 90 ; \text { SF36 = 36-item Short-Form Health Survey; DAS-A = Dysfunctional } \\
\text { Attitude Scale form A (we used a 17-item version); QwQ = Questionnaire without Questions. } \\
\text { The score range is shown in brackets. } \\
\text { a data unavailable: CCBT }(N=3) \text {, TAU }(N=4) \text {, and CCBT\&TAU }(N=3) \text {; }{ }^{b} \text { data unavailable: } \\
\text { CCBT }(N=7), \text { TAU }(N=17) \text {, and CCBT\&TAU }(N=6) ;{ }^{\circ} \text { data unavailable: CCBT }(N=7) \text {, TAU } \\
(N=8) \text {, and CCBT\&TAU }(N=4) \text {. }\end{array}$} \\
\hline
\end{tabular}




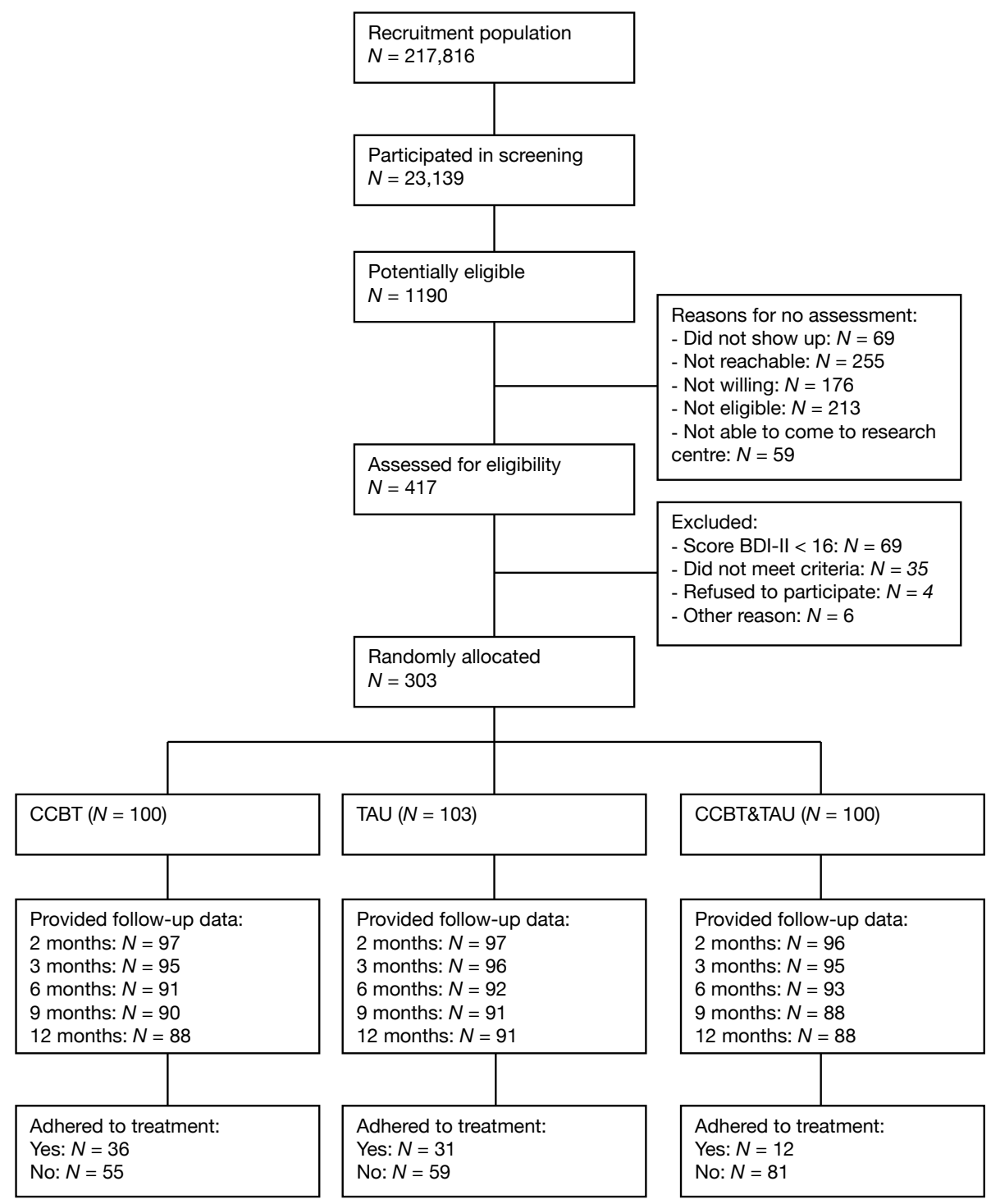

Figure 1 Flowchart

Note. CCBT = computerised cognitive behavioural therapy (i.e. CYL offered online without support); $\mathrm{TAU}=$ treatment as usual. 


\section{Moderators and predictors of depressive severity}

In table 2 significant predictors and moderators of BDI-II score at 12 months of follow-up are shown. Four variables moderated the effects of treatment on depressive severity at 12 months of follow-up (i.e. significant interaction effects) in the univariate models. At higher levels of extreme positive responding ( $Q w Q)$ and extreme negative responding (DAS-A), CCBT led to better outcomes than did TAU. Moreover, those that had a short-term decrease in extreme positive responding ( $Q w Q)$ did better in CCBT than in TAU. Patients with a parental psychiatric history responded better to CCBT\&TAU than to TAU.

Univariate analyses showed that seven variables predicted depressive severity at 12 months of follow-up irrespective of assigned treatment (i.e. main effects only). Patients who were employed at baseline had lower depression scores 12 months later. Patients who at baseline reported high levels of general psychopathology (SCL90), high depressive severity (BDI-II), and poorer general mental health (SF36) had higher depression scores 12 months later. Furthermore, short-term improvement on the BDIII, SCL90, and DAS-A perfectionism scale predicted better outcome regardless of treatment type.

The multivariate analyses revealed that baseline BDI-II, baseline SCL90, and short-term improvement on the BDI-II and SCL90, were significant predictors of depressive severity at 12 months of follow-up. Only extreme positive responding stayed in the model as moderator of treatment. This final model explained $45 \%$ of the variance in depressive severity $(F(9,265)=13.21 p<.001)$. 
Table 2 Significant moderators and predictors of treatment for BDI-II score at 12 months of follow-up $(N=267)$ : results from linear regression analyses

\begin{tabular}{|c|c|c|c|c|}
\hline & Variable & $B$ & $S E B$ & B \\
\hline \multicolumn{5}{|l|}{ Univariate } \\
\hline \multirow[t]{6}{*}{ Moderator } & \multicolumn{4}{|l|}{ CCBT relative to TAU: } \\
\hline & - Baseline QwQ -positive responding & -0.39 & 0.14 & $-0.22^{\star \star}$ \\
\hline & - Baseline DAS-A - negative responding & -0.35 & 0.17 & $-0.15^{\star}$ \\
\hline & $\begin{array}{l}\text { - Change in QwQ - positive responding } \\
\text { CCBT\&TAU relative to TAU: }\end{array}$ & -0.37 & 0.16 & $-0.22^{\star \star}$ \\
\hline & \multicolumn{4}{|l|}{ CCBT\&TAU relative to TAU: } \\
\hline & - Parental psychiatric history (yes) & -0.76 & 0.35 & $-0.19^{\star}$ \\
\hline \multirow[t]{7}{*}{ Predictor } & Employed (yes) & -0.52 & 0.25 & $-0.23^{\star}$ \\
\hline & Baseline BDI-II & 0.44 & 0.10 & $0.43^{\star \star \star}$ \\
\hline & Baseline SCL-90 & 0.24 & 0.11 & $0.23^{*}$ \\
\hline & Baseline SF36 - general mental health & -0.32 & 0.10 & $-0.32^{\star \star}$ \\
\hline & Improvement in BDI-II & -0.37 & 0.09 & $-0.37^{\star \star \star}$ \\
\hline & Improvement in SCL90 & -0.28 & 0.10 & $-0.29^{\star \star}$ \\
\hline & Improvement in DAS-A perfectionism & -0.27 & 0.10 & $-0.27^{\star \star}$ \\
\hline \multicolumn{5}{|l|}{ Multivariate } \\
\hline \multirow[t]{2}{*}{ Moderator } & \multicolumn{4}{|l|}{ CCBT relative to TAU: } \\
\hline & $\begin{array}{l}\text { - } \quad \text { Baseline } Q w Q \text {-positive responding } \\
\text { CCBT\&TAU relative to TAU: } \\
\text { - None }\end{array}$ & -0.33 & 0.12 & $-0.19^{\star \star}$ \\
\hline \multirow[t]{4}{*}{ Predictor } & Baseline BDI-II & 0.40 & 0.08 & $0.40^{\star \star \star}$ \\
\hline & Baseline SCL-90 & 0.21 & 0.08 & $0.20^{\star \star}$ \\
\hline & Improvement in BDI-II & -0.34 & 0.08 & $-0.35^{\star \star \star}$ \\
\hline & Improvement in SCL90 & -0.21 & 0.08 & $-0.21^{*}$ \\
\hline \multicolumn{5}{|c|}{$\begin{array}{l}\text { Note. CCBT = computerised cognitive behavioural therapy (i.e. CYL offered online without } \\
\text { support); TAU = treatment as usual; BDI-II = Beck Depression Inventory-II; QwQ }= \\
\text { Questionnaire without Questions; SCL-90 = Symptom Checklist } 90 ; \mathrm{SF} 36=36 \text {-item Short- } \\
\text { Form Health Survey. Results for continuous variables are reported as standard deviation } \\
\text { units. Each univariate model included the predictor/moderator, treatment type, and } \\
\text { predictor/moderator } x \text { treatment interaction, controlled for baseline BDI-II. Only significant } \\
\text { predictors and moderators }(p<.05) \text { are shown. Improvement was calculated as follows: } \\
t_{\text {baseline }}-t_{\text {month } 3} \text {. The relative contribution of these variables is subsequently evaluated in a } \\
\text { multivariate model using a backward elimination procedure. } \\
{ }^{*} p<.05 ;{ }^{* \star} p<.01 ;{ }^{* \star *} p<.001 \text {. }\end{array}$} \\
\hline
\end{tabular}




\section{Moderators and predictors of reliable change}

Table 3 presents the results of Cox regression analyses predicting reliable change (i.e. a decrease of at least 9 points on the BDI-II) during 12 months of follow-up. 142 (71\%) patients reliably changed, whereas 58 patients (29\%) were censored in the analyses.

In the univariate models, only one variable moderated treatment outcome. Those with a current diagnosis 'major depressive episode' were roughly three and a half times more likely to improve in the CCBT\&TAU group than in the TAU alone group.

Two variables predicted the outcome irrespective of treatment in the univariate models. Patients who were employed at baseline were two times more likely to improve than those without employment at that time. Furthermore, the likelihood of reliable change increased with higher levels of general mental health (SF36).

In the multivariate model $\left(\mathrm{X}^{2}(7)=31.03, p<.001\right)$, a current diagnosis 'major depressive episode' remained in the model as a moderator and general mental health (SF36) was still a predictor. Baseline BDI-II, which was added to the model as covariate, additionally emerged as a significant predictor of reliable change. That is, the likelihood for reliable change increased with almost one and a half for each standard deviation increase on the BDI-II at baseline.

Table 3 Significant moderators and predictors of treatment for reliable change during 12 months of follow-up $(N=267)$ : results from Cox regression analyses

\begin{tabular}{lll}
\hline & Variable & $H R(\mathrm{Cl} 95 \%)$ \\
\hline Univariate & & \\
Moderator & CCBT relative to TAU & \\
& $-\quad$ None & \\
& CCBT\&TAU relative to TAU & $3.74(1.25-11.19)^{\star}$ \\
Predictor & $-\quad$ Current depressive episode (yes) & $2.07(1.08-3.99)^{\star}$ \\
& Employed (yes) & $1.33(1.06-1.67)^{\star}$ \\
Multivariate & Baseline SF36 - general mental health & \\
& & \\
& CCBT relative to TAU & \\
& $-\quad$ None & $3.94(1.32-11.79)^{\star}$ \\
Predictor & CCBT\&TAU relative to TAU & $1.43(1.22-1.67)^{\star \star \star}$ \\
& Baseline BDI-II $\quad$ Current depressive episode (yes) & $1.32(1.13-1.54)^{\star \star \star}$ \\
\hline
\end{tabular}

Note. CCBT $=$ computerised cognitive behavioural therapy (i.e. CYL offered online without support); TAU = treatment as usual; BDI-II = Beck Depression Inventory-II; SF36 = 36-item Short-Form Health Survey. Results for continuous variables are reported as standard deviation units. Each univariate model included the predictor/moderator, treatment type, and predictor/moderator $\mathrm{x}$ treatment interaction, controlled for baseline BDI-II. Only significant predictors and moderators $(p<.05)$ are shown. The relative contribution of these variables, together with baseline BDI-II and intervention type, is subsequently evaluated in a multivariate model using a backward elimination procedure.

${ }^{\star} p<.05 ;{ }^{* \star} p<.01 ;{ }^{* * *} p<.001$. 


\section{Discussion}

\section{Main findings}

The present study explored pre-treatment and short-term improvement variables as potential moderators and predictors of 12 months of follow-up outcome among a sample of depressed individuals $(N=303)$ who participated in a randomised trial of unsupported online CCBT (i.e. Colour Your Life/CYL) and CCBT\&TAU versus TAU alone. Depressed individuals with higher pre-treatment levels of extreme negative and positive responding responded better to CCBT than to TAU, as were those with a short-term decrease in extreme positive responding. For those having a parental psychiatric history or a 'major depressive disorder' diagnosis CCBT\&TAU had a better effect than TAU. Predictors of outcome regardless of treatment type included current employment, low pre-treatment illness severity scores, and short-term improvement on clinical variables.

\section{Reflection on the findings}

Short-term improvement in depressive severity and general psychopathology both emerged as independent predictors of depressive severity at 12 months of follow-up irrespective of treatment. These findings make sense intuitively and have been supported by earlier research in various treatment modalities (see Mulder et al., 2006; Renaud et al., 1998; Seivewright et al., 1998; Van et al., 2008). Still, it is not easy to interpret this finding. It could imply that most of the change that is going to occur happens early or just that early response is a good proxy for later outcome. From a stepped-care perspective, it highlights the importance of systematic monitoring of depressive patients to identify those who require to step-up to a higher level of care. Renaud et al. (1998) suggested starting with a brief intervention to "wash out" what they call 'non-specific' responders.

More severely depressed individuals were more likely to end with high depression scores and to reliably change. The latter might seem odd, but can be explained by the fact that higher scores leave more room for improvement. We found similar results for general psychopathology (SCL90) and general mental health (SF36) that both measure feelings of depression and nervousness. This replicates the findings regarding Internet-based CBT (Andersson et al., 2004; Spek et al., 2008b), traditional face-to-face CBT (Jarrett et al., 1991), and primary care treatment in general (Wolf \& Hopko, 2008). Given the negative association between initial severity and outcome, it is necessary to adapt treatment strategies for these more severely depressed individuals. For this group of patients a combination of psychotherapy and antidepressant medication seems superior to single treatment (Friedman et al., 2004; Pampallona et al., 2004). However, we could not find this specific effect for our combination treatment of CCBT and TAU.

Patients who were unemployed or on sick leave at baseline had higher depression scores 12 months later. Poor employment status and the resulting financial losses or social isolation might be seen as a chronic stressor (Kessler, 1997). However, this finding could also have been a reflection of the impairment associated with the severity of the disorder, since it did not remain a predictor in our multivariate model.

The content of dysfunctional attitudes (i.e. perfectionism and dependency) did not emerge as a moderator in both CCBT conditions relative to TAU. This differs from findings in previous studies that showed that face-to-face CBT is a necessary treat- 
ment for depressed patients with high levels of dysfunctional attitudes (Hamilton \& Dobson, 2002; Jarrett et al., 1991). Recently, some researchers have argued that the form of dysfunctional thinking (i.e. an extreme response style), rather than the content, is associated with poor treatment outcome (Beevers et al., 2003; Petersen et al., 2007; Teasdale et al., 2001). We also tested this theory in our study, and found that CCBT led to better outcomes than TAU for extreme positive responders on the QwQ, extreme negative responders on the DAS-A, and for those who become less extremely positive on the $Q w Q$ over time. How can we explain these findings? First, we should comment on the measures used. Unlike the $Q w Q$, an extreme response on the DAS-A relates to the respondents' thought content. An extreme negative response to an item on the DAS-A might simply indicate an extreme level of dysfunctional thought content. Taken this way, the moderating effect of extreme negative responding on the DAS-A is in line with the traditional view that cognitive therapy is the most suitable option for depressed individuals with high dysfunctional attitudes (Hamilton \& Dobson, 2002). Second, we hypothesise that extreme positive responding reflects a latent disposition, such as optimism. We should keep in mind here that we measured extreme responding with an indirect measure of latent emotional tendencies, not specific to depression (i.e. QwQ). Optimists hold coping strategies that are approach-oriented (i.e. engagement and striving) rather than avoidance-oriented (i.e. disengagement and giving up) (Nes \& Segerstrom, 2006). Optimists (i.e. extreme positive responders) cope in active ways that are necessary for working independently through self-help material, such as CCBT. Third, patients who become less optimistic over time might be more responsive to a treatment format, such as CCBT, that facilitates greater opportunity to help challenge these changes. Moreover, during the first months of CCBT they might have learned strategies that help identify and tackle these changes. Finally, our findings on extreme responding clearly need replication and further examination, but they do suggest that an emphasis on the form of thinking might be particularly important in the treatment of depression. This view has already been adopted in mindfulness based treatments, in which the main aim is learning to decentre from negative thinking patterns (Teasdale et al., 2000). It is important to note that adherence to CCBT in our study was low (see de Graaf et al., in press-a), suggesting that perhaps even a small amount of unsupported online self-help has effects on the outcome for extreme responders.

We found that a combination treatment, i.e. both CCBT and TAU, had a better effect than TAU alone for those having a parental psychiatric history or a 'major depressive disorder' diagnosis. Parental psychiatric history has been associated with psychiatric comorbidity (de Graaf et al., 2004) and major depressive disorder has been associated with higher illness severity scores than sub-threshold depression (Kessler et al., 1997). Therefore, the current findings make sense in the way that both patient characteristics have been associated with vulnerability for more severe psychopathology that might warrant more intense treatments, such as combining treatment modalities. It should be noted that the effect of parental psychiatric history has been partialled out in the multivariate model by other more dominant predictors or moderators.

Variables that did not contribute to the prediction of treatment outcome in the current study are at least as informative as those that did, especially regarding those that have been reported in earlier studies. For example, it has been shown that gen- 
der and education were predictors of depressive severity one year later (Spek et al., 2008b). Like our study, earlier studies did not obtain significant effects for these variables either (Hamilton \& Dobson, 2002). Differences in study design and population might be accountable for this.

Finally, we should note that we found different patient characteristics that predicted the continuous (i.e. BDI-II score) and dichotomous outcome (i.e. reliable change). It is theoretically possible that different factors are related to the severity of the disorder and to reduction in depressive symptoms. It could also imply that what we found is not that robust and might largely be psychometric findings, since the baseline BDI-II score was the only consistent predictor.

Taken together, relative to the mounting evidence base for the effectiveness of various formats of CBT for depression, including computer-assisted CBT, we know only little to decide which treatment should be given to whom. Although the moderators found in the present study might help in making treatment decisions, replication is warranted. When the same patient characteristics are repeatedly found to moderate treatment outcome, this should eventually lead to the development of alternative treatment strategies.

\section{Methodological considerations}

The current study has several strengths. We had a relatively low attrition rate regarding data collection, so we feel confident that no biases occurred due to missing data. Furthermore, we were able to recruit patients from the general population. Unlike in samples selected in general practices or clinics, no biases occurred due to help seeking behaviour of patients and illness recognition by physicians, which is often a problem in depression (Paykel et al., 1997). However, our sample may have been too depressed in relation to the scope of the treatments in the current study, limiting the generalizability of our findings. Some other limitations of the present study should also be noted. First, all outcomes were assessed online and one might question the equality of computerised questionnaires and paper-and-pen versions. There are indications that they show similar psychometric properties (Carlbring et al., 2007a; Garb, 2007; Schulenberg \& Yutrzenka, 2001). Second, we relied on self-report measures at follow-up and, as a result, we have no information on actual diagnoses of depressive episodes after treatment. Third, as in previous prognostic studies on CCBT, predominantly Caucasian individuals participated. Future studies need to use samples representing different demographic and cultural backgrounds. Finally, the statistical analyses were performed a posteriori and power analyses were lacking for these tests. Together with the absence of clear hypotheses, this might have increased the chance of type I error. 


\section{Conclusion}

Unsupported online CCBT (i.e. Colour Your Life) may be a sufficient intervention for those that have an extreme positive response style, suggesting that CCBT may tap into latent tendencies that drive emotion. A combination treatment (i.e. CCBT and TAU) is the most suitable option for those with a parental psychiatric history and a major depressive disorder. Pre-treatment functioning and short-term improvement were predictors of outcome, regardless of the type of treatment. Those with the least impairment improve the most. Despite these differential responses to treatment, it remains to be seen whether our population would not have benefited more from specialist care. 


\section{Acknowledgements}

We thank Annie Hendriks, Sylvia Gerhards and Greet Kellens for their assistance during the study and Rosanne Janssen for the development of the infrastructure for online data-collection. The trial was financed by ZonMw (Netherlands Organisation for Health Research and Development; project number 945-04-417), research institute EPP and research institute CAPHRI. Municipalities Eijsden, Meerssen, Sittard-Geleen, Valkenburg and Maastricht sponsored the study. 

Chapter 10

\section{General discussion}




\section{What this discussion is about}

The current thesis addressed the assessment of features associated with depression, issues related to the cognitive theory of depression, and the cognitive behavioural treatment of depression via the Internet. In this final chapter these areas will be discussed, starting with an integration of the main findings followed by a reflection on their clinical implications. After addressing some methodological considerations of the studies, this chapter will end with recommendations for further research. Most attention will be paid to the treatment of depression, which was the central focus of this thesis.

\section{Integration of the main findings}

The most striking finding of this thesis came from the randomised trial, in which the (cost-) effectiveness of computerised cognitive behavioural therapy (CCBT) for depression was evaluated. During twelve months, we compared the costs and effects of (a) unsupported online CCBT with (b) treatment as usual (TAU) by a GP (c) and a combination of both treatments. Although CCBT was found to have a higher probability of being cost-effective than the other groups, we found no differences in effects between the three groups, which is in contrast to our study hypotheses. CCBT was not superior to TAU nor did the combination of CCBT and TAU have additional effects. Reductions in depressive severity were modest in all groups.

\section{A 'negative' trial}

It is common practice to refer to a trial that failed to confirm the expected findings, such as our trial, as a negative one. However, this suggests that the treatments were harmful or dangerous, which is rarely the case in psychological treatments. Moreover, all well-constructed trials contribute positively to scientific knowledge (see also Chalmers, 1985). From a researchers' point of view, contradictory trial findings might in fact be more exciting than trials confirming the study hypotheses. Unexpected outcomes generally lead to a more thorough and comprehensive examination of potential explanations, including patient characteristics (e.g. disease severity) and treatment related aspects (e.g. adherence and concomitant treatments). Disentangling the complex relationship between these aspects is difficult, especially afterwards. Future clinical trials in which these aspects are experimentally manipulated are required. Nevertheless, I will try to elaborate on these issues by integrating the findings described in the various studies in this thesis.

\section{Patient characteristics}

One explanation for the lack of effectiveness is that the tested interventions may not have been appropriate for the participating patients. The majority of our participants were diagnosed with 'major depressive disorder' (chapter 5), which has been associated with higher illness severity scores than milder forms of depression (chapter 3). These more severely depressed individuals were also less likely to do well in treatment (chapter 9). Patients with major depression may differ from their less severely depressed counterparts in the kind and intensity of treatment that is needed. Our participants may have been too depressed for the relatively mild nature of the interventions they were given. Should we have restricted the range of depressive severity by excluding these moderately to severely depressed individuals? We expected to in- 
clude less impaired patients than we eventually did, since participants were selected in the general population, not in a clinical setting, and were not being treated for their complaints. On the one hand, we could have excluded those who met criteria for a major depressive disorder to select more appropriate patients for the tested interventions. On the other hand, our findings teach us that CCBT is not especially suitable for this group of severely depressed individuals, which had not been tested before. More severe depression might warrant more intense treatments, such as combining treatment modalities (Pampallona et al., 2004). We found that CCBT and TAU combined resulted in better outcomes than TAU alone for those with a major depressive disorder (chapters 5, 6 and 9). Knowledge on how to categorise patients along the depression continuum can serve to improve treatment response rates (chapter 3 ).

\section{Treatment related aspects}

The low level of care received by all study participants may have reduced treatment effects. Treatment adherence was low in all interventions (chapters 5 and 8 ) and the use of health care services decreased during one year, despite the lack of substantial improvements (chapter 6). Although undertreatment could have masked potential effects of the interventions, treatment adherence was not found to differentiate the outcomes (chapter 5) and there were only few indications that frequent use of CCBT was related to a better treatment outcome (chapter 8 ). These findings confirm that many depressed patients in primary care are still being undertreated (see Kessler et al., 2003).

\section{Positive messages}

The thesis presents also a positive message. CCBT could indeed have potential as a strategy for reducing depressive symptoms. It was remarkable that with equal yet modest effects even a small portion of CCBT led to less health care consumption than usual GP care (chapter 6). Together with the finding that CCBT resulted in fewer costs than the other two treatment groups (chapter 7), it might be seen as an indication that CCBT could work - but under other circumstances. Furthermore, CCBT is potentially a feasible treatment option. Despite the fact that many patients dropped out during treatment, most of them completed the first session of CCBT (chapter 8). Moreover, patients rated CCBT as an acceptable treatment in terms of expectancy, credibility, and pre- and post-treatment satisfaction (chapter 8). Although the intervention was perhaps not ideal for our sample, these findings on usage and acceptability could be seen as indications for the feasibility of a computerised treatment approach.

\section{Cognitive vulnerability factors}

According to the cognitive model of depression, depressive states are characterised by a negative pattern of cognitions and dysfunctional beliefs (Beck, 1972; 1987). Successful cognitive (behavioural) treatment should be accompanied by modification or reduction of these dysfunctional beliefs (Beck, 1983). Dysfunctional beliefs have frequently been assessed by the Dysfunctional Attitude Scale form A (DAS-A). A revised version of the DAS-A proved useful to measure specific contents of dysfunctional beliefs as it has good psychometric qualities (chapter 2). In a cross-sectional study, we showed that the intensity of these dysfunctional beliefs increases when the severity of the disorder worsens (chapter 3). In the randomised trial, on the other hand, 
we found that CCBT did not reduce dysfunctional beliefs more than TAU (chapter 5) and that frequent use of CCBT was not associated with a reduction of dysfunctional beliefs (chapter 8). This might seem in contrast with the cognitive model of depression, but could be explained by the overall lack of effectiveness of the tested CCBT intervention. We did find an indication that CCBT is the most suitable treatment option for depressed patients with extremely high levels of dysfunctional attitudes (chapter 9). This is in line with the traditional view that modifying thought content is the mechanism through which cognitive therapy works.

In addition to studying the content of dysfunctional thinking, we were also interested in a relatively new concept that has been hypothesised to be a vulnerability factor for depressive relapse and recurrence: a form aspect of dysfunctional thinking (i.e. extreme responding) (see Beevers et al., 2003; Petersen et al., 2007; Teasdale et al., 2001). Extreme responding could be interpreted as a measure of the 'dichotomous thinking' construct from Beck's cognitive model of depression. This form of thinking refers to the tendency to evaluate experiences in two mutually exclusive categories, either negative or positive (Beck et al., 1979). However, high levels of extreme positive responses have actually been found in less severely depressed (chapter 3 ) and non-depressed individuals (Teasdale et al., 2001). An extreme positive response style might, therefore, not be a pathological way of thinking. Perhaps extreme responding might better be viewed as a way to conceptualise optimism and pessimism. In our trial, optimists (i.e. extreme positive responders) did better in CCBT than in usual GP care (chapter 9). Optimists use active coping strategies (Nes \& Segerstrom, 2006), which is necessary to use self-help material. Patients who become less optimistic over time were also more responsive to CCBT than usual GP care (chapter 9). CCBT might facilitate greater opportunity to help challenge such changes. As we were the first to show these effects, our findings need replication and further examination. Research on cognitive vulnerability factors can also help to improve adequate treatment for depression.

\section{Implications for clinical practice Bridging the treatment gap}

The huge gap between the demand on mental health care and the available supply was the leading ground for conducting the research described in this thesis. One way to bridge the treatment gap is by delegating routine aspects of effective treatment formats to a computer system. However, the main findings in this thesis suggest that we should be much more reluctant when it comes to the large-scale dissemination of computerised self-help treatments. Does this mean that we should stop treating depression via a computer system? The answer is no. Treatment decisions must be evidence based. There is accumulating evidence that CCBT can effectively reduce symptoms of depression (Kaltenthaler et al., 2008a). A single study failing to confirm this, should not be enough to abandon CCBT, especially not when no alternative treatments are readily available yet to a required extent. Moreover, failing to find significant and clinically meaningful effects, as in our study, should not wrongly be taken as evidence that the treatment is not useful in other settings, populations, and formats. The answer must then be: we should improve it. 


\section{Improving the effectiveness of online CCBT}

Improving the (cost-)effectiveness of a certain treatment is a difficult task and requires knowing what works best and what does not. There are recent indications that therapist support, which was absent in our trial, is one such crucial element in computerised self-help CBT (Spek et al., 2007a). However, it is not clear as to what it exactly is about the therapist's role that leads to better outcomes. It could be giving personalised feedback, being empathic, giving encouragement, providing flexibility in answering questions, or something else. A related unanswered question is by whom the therapist's role should be fulfilled. Within primary care, this role seems granted to nurse practitioners, social workers, or primary care psychologists. Further research is needed to define the therapist's role and identify other effective elements of CCBT, including specific therapy techniques (e.g. behavioural activation and restructuring cognitions), non-specific therapy components (e.g. homework; see chapter 8), and treatment context (e.g. setting, frequency, and duration).

The therapeutic effects of CCBT could also potentially be optimised by knowing what works for whom under which circumstances. This does not only inform us about which type of patient is most likely to benefit from CCBT, but could also serve to develop tailor-made CCBT to better meet specific needs and preferences of patients. The beauty of computer-based therapies is that information can be presented in various formats, languages, styles, and at various educational levels. Computertailored modules could be made for particular patient profiles (Christensen \& Griffiths, 2002), such as divorce, unemployment, specific symptoms, parental psychiatric history (chapter 9), or extreme thinking styles (chapter 9). Online screening could channel patients to specific modules (see Gega et al., 2005). So far, tailoring interventions has mainly been applied in changing health behaviour. For instance, a web-based tailored smoking cessation program had significant advantages over a non-tailored version of the online program (e.g. see Strecher et al., 2005). Future research should determine whether tailoring could improve the effectiveness of CCBT for depression and other common mental disorders.

After we better know what works in CCBT, efficient and effective implementation could improve the current suboptimal care for depressed patients. Full implementation in mental health care requires organizational changes. One strategy is to organise care in a stepwise manner. Computerised self-help could be a first element in a stepped-care approach (Bower \& Gilbody, 2005). There have only been few trials on the effectiveness of full stepped-care models. For instance, van Straten et al. (2006) showed that stepped-care is as effective as usual care and probably more efficient for mood and anxiety disorders. However, (computerised) self-help was not included as one of the steps in this study. More research on strategies for effective implementation is clearly needed.

\section{Do we need all that technology?}

Before the rise of the computers, written self-help materials - or bibliotherapy - have been shown to be effective treatments for depression (Cuijpers, 1997). If books work, do we need to bother with the advances in the digital world? No studies have directly compared both forms of standardised self-help CBT yet. It remains to be seen whether or not computerised self-help is more effective than a book. This could, for instance, be investigated by comparing the computer-based and manual-based "Coping with 
Depression" course. Both formats have already been developed (see Willemse et al., 2004 and this thesis). Nevertheless, we might not get around technology any longer. E-health is already an important feature of contemporary everyday life (Griffiths et al., 2007). As children are growing up surrounded by all kinds of digital tools, the movement from books to computerization seems inevitable for this generation.

\section{Methodological considerations}

As with all research, the current thesis was not without limitations, some of which need to be considered here as they apply to most studies described in this thesis. First of all, the recruitment of patients took more time and effort than estimated. This phenomenon is also known as Lasagna's law. Many factors could have affected patient recruitment in our trial, such as lack of motivation and unavailability of Internet. We might have included a selective group of secondary or tertiary care depressed patients, which may have reduced the external validity of the findings. Second, we have shown that it is possible to have a low attrition rate regarding data collection during one year of follow-up. Many previous studies using online measurements have had difficulties in keeping participants in the study. Especially when study withdrawal is selective, this can threaten the external and internal validity of the study. Our high response rate contributed to the quality of the collected data and the study was sufficiently powered to detect differences. This increased our confidence that our randomised trial was not a false negative (i.e. type II error), although this possibility cannot be ruled out. Third, in our randomised trial we did not include a waiting list control group. In the absence of a waiting list, we do not know whether the modest improvements were significantly better than doing nothing. We should keep in mind, however, that the background for our study was to improve patient care. In that sense, comparing a new intervention to usual care is most informative. Fourth, we relied almost solely on self-report measures. Despite the obvious advantages, self-rating scales do not measure diagnoses and they require self-insight, which might be limited in some patients.

\section{Directions for further research}

Here I will discuss some issues that might be important to consider for further research that may bring us closer to the optimal treatment for depression via the Internet.

\section{Other comparators}

A randomised clinical trial $(R C T)$ is regarded as the gold standard for evaluating the efficacy and effectiveness of treatments. Despite this and the numerous publications on CCBT, only few RCTs have been conducted that examined the efficacy or effectiveness of CCBT for adult unipolar depression, comparing it to treatment as usual (Clarke et al., 2002; Clarke et al., 2005; de Graaf et al., in press-a; Proudfoot et al., 2004), therapist-administered CBT (Selmi et al., 1990), a waiting list (Andersson et al., 2005; Selmi et al., 1990; Warmerdam et al., 2008), an information website (Christensen et al., 2004a), web-based problem solving (Warmerdam et al., 2008), and attention placebo (Christensen et al., 2004a). To facilitate treatment decision making, future research should include other comparative treatments that are effective or widely 
used for depression, such as bibliotherapy, antidepressant medication, and face-toface psychotherapy, including cognitive behavioural therapy, behavioural activation, interpersonal psychotherapy, and psychodynamic interventions. Since combining pharmacotherapy and CBT for depression is widely practiced in the community, it is worth examining the effects of combined CCBT and antidepressants as well. It might increase the compliance to both treatments. It should be noted that we included a combination treatment of CCBT and usual GP care in our trial. Although GP care can include antidepressant medication, GPs will only start pharmacotherapy in case of severe or ongoing dysfunction. Patients assigned to the combination treatment in our study were therefore not structurally prescribed antidepressant medication.

\section{Other formats}

The only evidence for the effectiveness of computerised therapy for depression is based upon CBT. This seems justified, since many studies have shown that face-toface CBT is more effective than other forms of psychotherapy (Butler et al., 2006). However, in a recent meta-analysis it was concluded that interpersonal psychotherapy, not CBT, was slightly more efficacious than other psychological treatments $(d=$ 0.21) (Cuijpers et al., 2008). Computerizing other effective face-to-face psychotherapies is an unexplored and interesting area or research. One such study has recently been conducted, showing that Internet-based CBT and Internet-based problem-solving were equally efficacious (Warmerdam et al., 2008). Since support by a therapist is largely absent in computerised therapies, comparing different formats can additionally facilitate research on specific therapy factors. The core components of a therapy can more easily be dismantled in computerised therapy than in face-to-face psychotherapy. This could contribute to the discussion on the 'Dodo Bird Verdict' that states that all psychotherapies are equally effective because of common therapy factors (Luborsky et al., 2002).

\section{Mechanisms of change}

In psychotherapy research, the study of the mechanisms (i.e. processes or events) through which therapeutic change takes place is still in its infancy. This holds true even more for computerised therapy. It is unknown by which mechanisms CCBT exerts its therapeutic change. Understanding why and how CCBT works can help to optimise treatment effects in subsequent studies. Identifying mediators is a first step in understanding how a treatment works (Kazdin, 2007; Murphy et al., 2009). Mediators are statistically associative links between treatment and outcome. They should be distinguished from moderators, which influence the extent to which change occurs (Kraemer et al., 2002). Mediational analyses should be followed by experimental studies in which the proposed mediators are manipulated and varied across groups (Kazdin, 2007; Murphy et al., 2009). There are several potential candidates that can serve as a mechanism of change in CCBT. The choice of variables to be investigated should have some theoretical basis. Changes in dysfunctional beliefs (cognitive theory) and coping behaviour (behavioural theory) are theoretically the mechanisms by which CCBT reduces depressive symptoms. The form of dysfunctional thinking also needs to be considered as a potential mechanism (chapters 3 and 9). Common therapy factors (e.g. homework, see chapter 8) as well as factors that are relatively unique to self-help (e.g. learned resourcefulness) are also worth examining. 


\section{Long-term follow-up}

An important limitation of many previous studies on the effects of CCBT is their relatively short follow-up period. Depression is associated with high risk of relapse, recurrence, and chronicity (Judd, 1997; Judd et al., 2000; Keller \& Boland, 1998). It has been shown that CBT can reduce rates of relapse and recurrence (Butler et al., 2006; Hollon et al., 2006; Vittengl et al., 2007). This relapse prevention effect can last for several years (Paykel et al., 2005). It could be assumed that CCBT has similar potency in preventing relapse. It is therefore worthwhile to specifically study the long-term prevention effects as we have tried in chapter 6 . Although relapse rates did not significantly differ between the groups in our study, it is not inconceivable that the effects will only just become visible in a few years. Therefore, the follow-up of the original trial has been extended up to 36 months after randomisation. Future studies additionally need to develop and evaluate specific computerised modules to maintain and stimulate improvements made during the therapy. Such computerised treatment strategies aimed to prevent relapse could also be used after formal face-to-face psychotherapy or pharmacotherapy has ended.

\section{On the digital road to recovery?}

Earlier research has laid the first stones of the digital road to recovery in depression. This thesis has put up some barriers for building this road. However, failures should be taken as lessons from which we can learn how to build a more solidly paved digital road. Personally, I do expect that in the future many depressed patients will walk on the digital road to recovery successfully. Until then, further scientific research need to grow insight into improving the treatment of depression via the Internet. This is an interesting yet difficult challenge. 


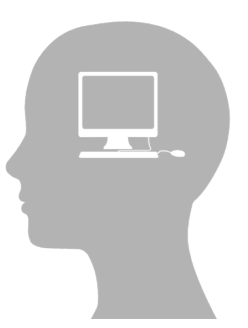

Summary 
Major depression is a common mental health problem in the general population and is associated with a substantial impact on quality of life and societal costs. Given this impact, effective treatment is essential. Cognitive behavioural therapy (CBT) is an effective treatment for depression. However, only a limited number of depressed patients in primary care receive appropriate care. The Internet could be an acceptable, feasible, and effective new mode of delivery for CBT. Delegating therapy components to a computer system can potentially reduce the undertreatment of depression in primary care. This was the leading ground for conducting the research described in this thesis.

Chapter 1 provides the general introduction to the contents of this thesis. It elaborates on the importance of further research on the effectiveness of computerised treatments for depression. A rough sketch is given of the history of the use of computers in mental health care. The many computer systems that have been used, are described. The advantages and disadvantages of computerised CBT (CCBT) are mentioned. Previous research that already contributed to the evidence base for CCBT for depression is discussed. The theoretical origins of CBT are highlighted. The chapter ends with a short outline of the main aims and the studies presented in this thesis.

Chapter 2 describes the results from the first cross-sectional study in this thesis. In this study the psychometric properties of the Dysfunctional Attitude Scale form A (DAS-A) were examined. The DAS-A is designed to measure the presence and intensity of dysfunctional attitudes, which constitute a cognitive vulnerability factor for depression. However, the total score on the DAS-A might be too general to adequately test the cognitive theory of depression. Therefore, several studies have aimed to investigate the factor structure of the DAS-A. These studies yielded mixed results due to methodological shortcomings. The current study tested these previously proposed factor models in a large community sample $(N=8,960)$ using confirmatory factor analysis, which is a stringent procedure to the test the factor structure of an instrument. It was shown that all seven competing models had a good fit to the data. Two factors have emerged consistently across six of these models: 'dependency' and 'perfectionism/performance evaluation'. Therefore, a two-factor solution of the DAS-A was retained consisting of 17 items. These factors demonstrated good reliability and convergent construct validity. The 17-item DAS-A proved useful in defining and measuring dysfunctional attitudes and was therefore used in many subsequent studies described in this thesis.

Chapter 3 includes the findings from the second cross-sectional study. In this study the relationship between minor and major depression was explored. Ongoing debate has focused on whether minor depression is a discrete diagnostic category or part of continuum that differs from major depression only in severity. One technique to address the continuum issue is to compare characteristics of minor depression with those of major depression. Subtypes on the continuum should be similar in demographic factors, whereas factors that mark the severity should be different. Therefore, we compared these factors between minor depression (i.e. 2 - 4 symptoms), major depression with 5 to 6 symptoms, and major depression with 7 to 9 symptoms using a large community sample $(N=1,129)$. We had a special focus on features related to dysfunctional thinking. We did not only explore whether minor and major depression share features related to the content of dysfunctional thinking (i.e. scores on the DAS-A), but we also focused on its form (i.e. extreme responding). The findings of this 
study mainly suggested that minor and major depression should be considered as parts of the same continuum. Not only the clinical characteristics, but also dysfunctional cognitive factors seemed to act in synchrony with the depression continuum. Both the content and the form of dysfunctional thinking seemed to play a important role in depression.

Chapter 4 describes the design of the randomised trial that evaluated the clinical and costs-effectiveness of unsupported online CCBT for depression in primary care. We compared (a) unsupported online CCBT with (b) treatment as usual (TAU) by a GP and (c) CCBT in combination with TAU. It was hypothesised that CCBT would be more (cost-)effective than TAU and that the combination of CCBT and TAU would be more (cost-)effective than CCBT alone. Recruitment of 303 depressed patients (aged 18-65) took place in the general population by means of a large-scale Internetbased screening. Eligible participants were randomly allocated to one of the three interventions. Participants allocated to CCBT and the combination of CCBT and TAU were given login codes for Colour Your Life: an online, multimedia, interactive, selfhelp computer program based on the principles of CBT. The program was offered without professional support. Participants allocated to TAU and the combination of CCBT and TAU were advised to contact their own GP for treatment of their depressive complaints. GPs were advised to follow Dutch depression guidelines. Baseline assessment took place at Maastricht University before randomisation. Follow-up assessment took place at home via the Internet respectively at two, three, six, nine, and twelve months after inclusion. The primary outcome measure of the clinical evaluation was the severity of depression. Other outcomes included psychological distress, social functioning, and dysfunctional attitudes. The economic evaluation was performed from a societal perspective, in which all costs were related to clinical effectiveness and to health-related quality of life. Costs were measured on a monthly basis. All subsequent chapters of this thesis present findings that were obtained during this trial.

Chapter 5 presents the findings regarding the six-month clinical effectiveness of unsupported online CCBT for depression in primary care. In contrast to our hypotheses, no statistically significant or clinically meaningful differences in outcome between the three interventions were found in the intention-to-treat and per-protocol analyses. This means that unsupported online CCBT did not outperform usual GP care and that the combination of both did not have additional effects. Moreover, the decrease in depressive symptoms was moderate in all three interventions. We suggested that the intensity of these primary care interventions may have been too low for our sample of moderately to severely depressed patients.

Chapter 6 reports the clinical outcomes from the randomised trial after twelve months. Long-term effects have rarely been reported on CCBT. Despite the lack of effectiveness as shown in the previous chapter, we also tested whether unsupported online CCBT can prevent depressive relapse. Since the level of health care consumption can influence the long-term effects and depressive relapse, we additionally examined the concomitant use of antidepressant medication and treatment in specialist mental health care. The one-year follow-up results looked fairly similar to the results at six-months of follow-up. Reductions in depressive severity remained comparable over time between the three interventions. We were unable to find a relapse prevention effect favouring CCBT. The overall improvements were rather modest. This might be attributed to the high amount of undertreatment in our trial. Nevertheless, we found 
that with equal effects between the three interventions, CCBT led to reduced health care consumption.

Chapter 7 presents the economic evaluation of CCBT for depression in primary care. CCBT resulted in lower societal costs during twelve months of follow-up than TAU and the combination of CCBT and TAU. Various cost-effectiveness and costutility scenarios were evaluated. They generally showed that CCBT has a higher probability of being the best treatment strategy than the other two interventions. However, differences between the three interventions were only related to cost aspects and not to improvement in depression and quality of life. Although the findings from this economic evaluation seemed in favour of CCBT, it can still be argued whether this treatment is suitable for moderately to severely depressed patients.

Chapter 8 describes an exploratory study that aimed to have a closer look at usage and acceptability (i.e. expectancy, credibility, and satisfaction) of the CCBT intervention and at how these factors were related to treatment outcome (i.e. reduction in depressive symptoms and dysfunctional attitudes). Since we were unable to confirm the previously reported high effectiveness of CCBT, such information is especially important because it can aid to optimise the intervention for future use. Regarding usage of CCBT, we found that many patients started using the intervention, but that only few completed all sessions. Women and highly educated patients were statistically most likely to take up CCBT. Frequent use of CCBT was positively associated with depressive improvement on the short-term, whereas only homework compliance was related to long-term depressive improvement. The number of logins was associated with short-term change in dysfunctional attitudes, while none of the usage indices were associated with long-term change in dysfunctional attitudes. We should be cautious in interpreting these findings, as the associations between use of CCBT and improvement are merely correlational. Regarding the acceptability of CCBT, we found that patients had positive expectations before the start of the intervention, but that after three months their opinion was relatively neutral. Positive treatment expectancy, but not credibility, was related to the long-term improvement in both depression and dysfunctional attitudes. It was concluded that CCBT might be a feasible and acceptable treatment for depression, but means to improve treatment adherence are needed for moderately to severely depressed individuals.

Chapter 9 shows the study that aimed to investigate moderators and predictors of depression status at one-year of follow-up among the participants in the randomised trial. Studies on moderators and predictors of outcome in CCBT are scarce, but this could help facilitate optimal treatment selection, improve depression care, and prevent poor treatment response. In the current study, potential moderators and predictors were demographic, clinical, cognitive, and short-term improvement variables. The results showed that those with higher levels of extreme responding had a better outcome in CCBT compared to TAU. CCBT might tap into latent emotional tendencies that stimulate improvement. The combination of CCBT and TAU, on the other hand, might be the most suitable option for those with severe vulnerability characteristics, i.e. a parental psychiatric history and a 'major depressive disorder' diagnosis. It was found that those with the least impairment improved the most regardless of the treatment received.

Chapter 10 offers a general discussion of this thesis by integrating and reflecting on the main findings. To be able to understand the trial findings, the complex relation- 
ship between patient characteristics and treatment related aspects needs to be disentangled, which is difficult to do afterwards. Possible implications for clinical practice are presented, methodological limitations are discussed, and recommendations for further research on the treatment of depression via the Internet are given. The main message of this chapter, and of this thesis as whole, constitutes that, although CCBT holds promise for the future, we should be much more reluctant when it comes to the large-scale dissemination of computerised self-help treatments for depression. 



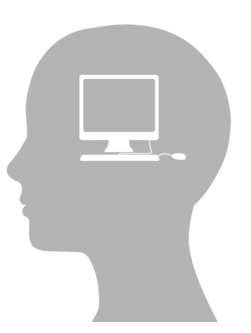

\section{Samenvatting}

(Dutch summary) 
Depressie kent een hoge prevalentie in de algemene bevolking en is geassocieerd met een aanzienlijke ziektelast. De economische gevolgen voor de maatschappij zijn substantieel. Effectieve behandeling van de klachten is daarom van essentieel belang. Cognitieve gedragstherapie (CGT) is een effectieve behandelmethode voor depressie. Veel mensen met een depressie krijgen in de eerstelijnszorg echter geen adequate behandeling. Via het internet kan CGT op een acceptabele, uitvoerbare en effectieve manier worden aangeboden. Door werkzame therapiecomponenten toe te wijzen aan een computersysteem kan de onderbehandeling van depressie in de eerstelijnszorg verminderd worden. Dit was aanleiding voor het onderzoek dat beschreven is in dit proefschrift.

Hoofdstuk 1 is de algemene inleiding op de inhoud van dit proefschrift. Het gaat in op het belang van onderzoek naar de effectiviteit van gecomputeriseerde behandelingen voor depressie. Er staat een korte schets van de geschiedenis van het gebruik van computers in de geestelijke gezondheidszorg. De vele gebruikte computersystemen komen ook aan de orde. De voor- en nadelen van gecomputeriseerde CGT (CCGT) worden genoemd. Eerder onderzoek dat bijdroeg aan de 'evidence base' voor CCGT voor depressie wordt bediscussieerd. De theoretische achtergrond van CGT wordt geschetst. Het hoofdstuk eindigt met een kort overzicht van de voornaamste doelen en de gepresenteerde studies in dit proefschrift.

Hoofdstuk 2 beschrijft de resultaten van de eerste cross-sectionele studie van dit proefschrift. In deze studie werden de psychometrische eigenschappen van de Dysfunctional Attitude Scale form A (DAS-A) onderzocht. De DAS-A is ontwikkeld om de aanwezigheid en intensiteit van disfunctionele attitudes te meten. Deze vormen een cognitieve kwetsbaarheidsfactor voor depressie. Echter, de totaalscore van de DAS-A is mogelijk te algemeen voor onderzoek naar de cognitieve theorie van depressie. Vandaar dat diverse voorgaande studies de factorstructuur van de DAS-A hebben onderzocht. Deze studies toonden echter gemengde resultaten vanwege methodologische beperkingen. De huidige studie heeft deze eerder voorgestelde factormodellen getest in een grote steekproef uit de algemene bevolking $(N=8960)$ door middel van confirmatieve factoranalyse: een strikte procedure om de factorstructuur van een instrument te testen. De zeven concurrerende modellen toonden een goede fit met de data. Twee factoren kwamen tevoorschijn bij zes van deze modellen: 'afhankelijkheid' en 'perfectionisme/prestatie evaluatie'. Op grond hiervan werd een model van twee factoren gehandhaafd bestaande uit 17 items. Deze factoren bleken een goede betrouwbaarheid en convergente constructvaliditeit te hebben. Het was aangetoond dat de 17-item DAS-A bruikbaar is bij het bepalen en meten van disfunctionele attitudes. De 17-item DAS-A werd om die reden gebruikt in de meeste volgende studies zoals beschreven in dit proefschrift.

Hoofdstuk 3 geeft de bevindingen van de tweede cross-sectionele studie weer. In deze studie is de relatie tussen minor en major depressie onderzocht. Er is een discussie gaande of minor depressie een discrete diagnostische categorie is of onderdeel vormt van een continuüm en alleen verschilt van major depressie qua ernst. Eén techniek om de continuüm kwestie te onderzoeken is het vergelijken van kenmerken van respectievelijk minor en major depressie. Subtypes op het continuüm zouden dezelfde demografische kenmerken moeten hebben, terwijl kenmerken die de ernst markeren zouden moeten verschillen. Daarom hebben we de kenmerken van minor depressie ( 2 - 4 symptomen), major depressie met 5 tot 6 symptomen en 
major depressie met 7 tot 9 symptomen vergeleken, gebruikmakend van een grote steekproef uit de algemene bevolking $(N=1129)$. We hebben ons hierbij speciaal gericht op factoren die gerelateerd zijn aan disfunctioneel denken. We hebben niet alleen onderzocht of minor en major depressie kenmerken deelden met betrekking tot de inhoud van disfunctioneel denken (scores op de DAS-A), maar we hebben ons ook gericht op de vorm van het denken (een extreme responsstijl). De bevindingen van deze studie toonden voornamelijk dat minor en major depressie gezien moeten worden als onderdelen van hetzelfde continuüm. Niet alleen de klinische kenmerken, maar ook factoren met betrekking tot disfunctioneel denken leken zich te gedragen in overeenstemming met het depressie continuüm. Zowel de inhoud als de vorm van disfunctioneel denken bleken een belangrijke rol te spelen bij depressie.

Hoofdstuk 4 beschrijft de opzet van de gerandomiseerde trial. In deze trial onderzochten we de klinische en kosteneffectiviteit van onbegeleide online CCGT voor depressie in de eerstelijnszorg. We vergeleken (a) onbegeleide online CCGT met (b) gebruikelijke zorg door de huisarts (TAU) en (c) CCGT in combinatie met TAU. We verwachtten dat CCGT (kosten)effectiever zou zijn dan TAU en dat de combinatie van CCGT en TAU (kosten)effectiever zou zijn dan alleen CCGT. De rekrutering van 303 depressieve patiënten (leeftijd 18-65 jaar) vond plaats in de algemene bevolking door middel van een grootschalige screening via het internet. Geschikte deelnemers werden willekeurig toegewezen aan een van de drie interventies. Deelnemers in de CCGT en in de combinatiegroep ontvingen inlogcodes voor 'Kleur Je Leven': een online, multimedia, interactief, zelfhulpprogramma, gebaseerd op de principes van CGT. Het programma werd aangeboden zonder professionele begeleiding. Deelnemers in de TAU en in de combinatiegroep werden voor de behandeling van depressieve klachten geadviseerd contact op te nemen met hun huisarts. Huisartsen kregen het advies om de Nederlandse depressierichtlijnen te volgen. De baseline meting vond plaats op de Universiteit Maastricht voor randomisatie. De vervolgmetingen werden respectievelijk twee, drie, zes, negen en twaalf maanden na aanvang van de studie thuis uitgevoerd via het internet. De primaire uitkomstmaat voor de klinische evaluatie was de ernst van depressie. Andere uitkomsten waren psychologische stress, sociaal functioneren en disfunctionele attitudes. We voerden de economische evaluatie uit vanuit een maatschappelijk perspectief, waarbij alle kosten werden gerelateerd aan de klinische effectiviteit en aan gezondheidsgerelateerde kwaliteit van leven. Kosten werden maandelijks gemeten. Alle volgende hoofdstukken van dit proefschrift beschrijven de resultaten die gevonden zijn gedurende deze trial.

Hoofdstuk 5 presenteert de bevindingen met betrekking tot de klinische effectiviteit van onbegeleide online CCGT voor depressie in de eerstelijnszorg gedurende zes maanden. In tegenstelling tot onze hypothesen vonden we geen statisch significante of klinisch betekenisvolle verschillen tussen de drie interventies in de 'intentionto-treat' en 'per-protocol' analyses. Dit betekent dat onbegeleide online CCGT niet effectiever was dan gebruikelijke huisartsenzorg en dat de combinatie van beide geen extra effecten had. Bovendien bleek de vermindering van depressieve symptomen matig in de drie interventies. We menen dat de intensiteit van deze eerstelijns behandelingen mogelijk te laag was voor onze steekproef van matig tot ernstig depressieve patiënten.

Hoofdstuk 6 rapporteert de klinische uitkomsten van de trial na twaalf maanden. Lange termijn effecten met betrekking tot CCGT zijn nauwelijks gerapporteerd. 
Ondanks het gebrek aan effectiviteit, zoals omschreven in het vorige hoofdstuk, onderzochten we ook of onbegeleide online CCGT depressieterugval kan voorkomen. Omdat de mate van gezondheidszorgconsumptie de lange termijn effecten en de depressieterugval kan beïnvloeden, onderzochten we ook het bijkomend gebruik van antidepressieve medicatie en specialistische behandeling in de geestelijke gezondheidszorg. De follow-up resultaten na een jaar zijn vergelijkbaar met de resultaten na zes maanden follow-up. Verminderingen in de ernst van depressie bleven in de tijd vergelijkbaar tussen de drie interventies. We konden geen terugvalpreventie effect vinden ten gunste van CCGT. In het algemeen waren de verbeteringen bescheiden. Dit kan worden toegeschreven aan de hoge mate van onderbehandeling in onze trial. Desondanks vonden we dat met gelijke effecten van de drie interventies CCGT leidde tot verminderd gebruik van gezondheidszorg.

Hoofdstuk 7 presenteert de economische evaluatie van CCGT voor depressie in de eerstelijnszorg. CCGT leidde tot minder maatschappelijke kosten gedurende twaalf maanden follow-up dan TAU en de combinatie van CCGT en TAU. Verscheidene kosteneffectiviteit en kostenutiliteit scenario's zijn geëvalueerd. De evaluatie laat zien dat CCGT over het algemeen een grotere kans heeft de beste behandelmogelijkheid te zijn dan de twee andere interventies. Verschillen tussen de drie interventies zijn echter alleen gerelateerd aan kosten en niet aan verbetering in depressie of kwaliteit van leven. Ondanks dat de bevindingen van de economische evaluatie ten gunste van CCGT lijken, kan worden betwist of deze behandeling wel geschikt is voor matig tot ernstig depressieve patiënten.

Hoofdstuk 8 beschrijft een exploratieve studie met als doel het nader bekijken van het gebruik en de aanvaardbaarheid (d.w.z. verwachting, geloofwaardigheid en tevredenheid) van de CCGT interventie en van de wijze waarop genoemde factoren gerelateerd zijn aan de behandeluitkomst (d.w.z. vermindering van depressieve symptomen en disfunctionele attitudes). Omdat we de eerder gerapporteerde hoge effectiviteit van CCGT niet konden bevestigen, is zulke informatie belangrijk. Deze kan bijdragen aan de verbetering van de interventie voor toekomstig gebruik. Met betrekking tot het gebruik van CCGT vonden we dat veel patiënten een start maakten met de interventie, maar dat slechts weinigen alle sessies afrondden. Vrouwen en hoogopgeleiden bleken statistisch gezien het meest waarschijnlijk om te starten met CCGT. Frequent gebruik van CCGT was positief geassocieerd met een verbetering van depressie op de korte termijn, terwijl alleen het maken van huiswerk gerelateerd was aan verbetering van depressie op de lange termijn. Het aantal login's was geassocieerd met korte termijn verandering in disfunctionele attitudes, terwijl geen enkele gebruiksindicator geassocieerd was met lange termijn verandering in disfunctionele attitudes. Een voorzichtige interpretatie van deze resultaten is geboden, omdat de associaties tussen het gebruik van CCGT en verbetering in depressie en disfunctionele attitudes voornamelijk correlationeel zijn. Met betrekking tot de aanvaardbaarheid van CCGT kan gezegd worden dat patiënten positieve verwachtingen hadden voor de start van de interventie, maar dat na drie maanden hun mening betrekkelijk neutraal was. Een positieve verwachting van de behandeling, maar niet geloofwaardigheid, was gerelateerd aan verbetering in depressie en disfunctionele attitudes op de lange termijn. Er werd geconcludeerd dat CCGT wellicht een uitvoerbare en acceptabele behandeling is van depressie, maar dat manieren om de therapietrouw te vergroten nodig zijn voor matig tot ernstig depressieve patiënten. 
Hoofdstuk 9 toont de studie met als doel de moderators en voorspellers voor de status van depressie na een jaar van follow-up te onderzoeken bij de deelnemers aan de gerandomiseerde trial. Studies over moderators en voorspellers van de uitkomst bij CCGT zijn schaars, terwijl dit de optimale selectie van behandelingen gemakkelijker zou maken, de zorg voor depressie zou verbeteren en een slechte respons op de behandeling zou voorkomen. In de onderhavige studie bestonden potentiële moderators en voorspellers uit demografische, klinische en cognitieve factoren, en variabelen met betrekking tot de verbetering op korte termijn. De resultaten toonden dat degenen met hogere niveaus van een extreme responsstijl een betere uitkomst hadden in CCGT dan in TAU. CCGT boort wellicht latent emotionele neigingen aan die verbetering stimuleren. De combinatie van CCGT en TAU is daarentegen wellicht de beste optie voor degenen met ernstige kwetsbaardheidskenmerken, namelijk een ouderlijke psychiatrische voorgeschiedenis en een diagnose 'depressieve stoornis'. We vonden dat degenen met de minste beperkingen het meest verbeterden ongeacht de ontvangen behandeling.

Hoofdstuk 10 biedt een algemene discussie van dit proefschrift door de integratie van en reflectie op de belangrijkste bevindingen. Om de resultaten uit de trial te begrijpen moet de complexe relatie tussen kenmerken van de patiënt en aspecten gerelateerd aan de behandeling ontrafeld worden, hetgeen achteraf moeilijk is. Mogelijke implicaties voor de klinische praktijk worden gepresenteerd, methodologische beperkingen worden bediscussieerd en aanbevelingen voor toekomstig onderzoek naar de behandeling van depressie via het internet worden gegeven. De kernboodschap van dit hoofdstuk, alsmede van het gehele proefschrift, is dat enerzijds CCGT veelbelovend lijkt voor de toekomst en dat anderzijds grote voorzichtigheid geboden is wanneer het gaat om de disseminatie van gecomputeriseerde zelfhulp voor depressie op grote schaal. 



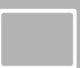

$2+1$

References 
Aaronson, NK, Muller, M, Cohen, PD, Essink-Bot, ML, Fekkes, M, Sanderman, R, Sprangers, MAG, te Velde, A \& Verrips, E (1998). Translation, validation, and norming of the Dutch language version of the SF-36 Health Survey in community and chronic disease populations. J Clin Epidemiol, 51, 1055-1068.

Addis, ME \& Jacobson, NS (2000). A closer look at the treatment rationale and homework compliance in cognitive-behavioral therapy for depression. Cognitive Therapy and Research, $25,313-326$

Algina, J, Penfield, RD \& Keselman, HJ (2005). An alternative to Cohen's standardized mean difference effect size: a robust parameter and confidence interval in the two independent groups case. Psychol Methods, 10, 317-328.

Alloy, LB, Abramson, LY, Hogan, ME, Whitehouse, WG, Rose, DT, Robinson, MS \& Kim, RS (2000). The Temple-Wisconsin cognitive vulnerability to depression project: lifetime history of axis I psychopathology in individuals at high and low cognitive risk for depression. $J$ Abnorm Psychol, 109, 403-418.

Alloy, LB, Abramson, LY, Whitehouse, WG, Hogan, ME, Panzarella, C \& Rose, DT (2006). Prospective incidence of first onsets and recurrences of depression in individuals at high and low cognitive risk for depression. J Abnorm Psychol, 115, 145-156.

Anderson, P, Jacobs, C \& Rothbaum, BO (2004). Computer-supported cognitive behavioral treatment of anxiety disorders. J Clin Psychol, 60, 253-267.

Andersson, G, Bergström, J, Holländare, F, Carlbring, P, Kaldo, V \& Ekselius, L (2005). Internetbased self-help for depression: randomised controlled trial. Br J Psychiatry, 187, 456461.

Andersson, G, Bergström, J, Holländare, F, Ekselius, L \& Carlbring, P (2004). Delivering cognitive behavioural therapy for mild to moderate depression via the Internet: predicting outcome at 6-month follow-up. Verhaltenstherapie, 14, 185-189.

Andersson, G \& Cuijpers, $P$ (2008). Pros and cons of online cognitive-behavioural therapy. $\mathrm{Br} \mathrm{J}$ Psychiatry, 193, 270-271.

Arnau, RC, Meagher, MW, Norris, MP \& Bramson, R (2001). Psychometric evaluation of the Beck Depression Inventory-II with primary care medical patients. Health Psychol, 20, 112-119.

Arrindell, WA \& Ettema, H (1981). Dimensionele sctructuur, betrouwbaarheid en validiteit van de Nederlandse bewerking van de Symptom Checklist (SCL-90); gegevens gebaseerd op een fobische en een 'normale' populatie. Nederlands Tijdschrift voor de Psychologie, 36, 77-108.

Backenstrass, M, Frank, A, Joest, K, Hingmann, S, Mundt, C \& Kronmüller, KT (2006). A comparative study of nonspecific depressive symptoms and minor depression regarding functional impairment and associated characteristics in primary care. Compr Psychiatry, 47, 35-41.

Beard, C \& Amir, N (2008). A multi-session interpretation modification program: changes in interpretation and social anxiety symptoms. Behav Res Ther, 46, 1135-1141.

Beck, AT (1967). Depression: clinical, experimental and theoretical aspects. New York: Hoeber.

Beck, AT (1972). Depression: causes and treatment. Philadelphia: University of Pennsylvania Press.

Beck, AT (1983). Cognitive therapy of depression: new perspectives. In Treatment of depression: old controversies and new approaches (eds PJ Clayton \& JE Barrett), pp. 265-290. New York: Raven Press.

Beck, AT (1987). Cognitive models of depression. Journal of Cognitive Psychotherapy: An International Quarterly, 1, 5-37.

Beck, AT, Brown, G, Steer, RA \& Weissman, AN (1991). Factor analysis of the dysfunctional attitude scale in a clinical population. Psychological Assessment, 3, 478-183.

Beck, AT, Guth, D, Steer, RA \& Ball, R (1997). Screening for major depression disorders in medical inpatients with the beck depression inventory for primary care. Behav Res Ther, 35, 785-791.

Beck, AT, Rush, AJ, Shaw, BF \& Emery, G (1979). Cognitive therapy of depression. New York: Guildford Press.

Beck, AT, Steer, RA, Ball, R \& Ranieri, WF (1996). Comparison of Beck Depression Inventories-IA and -II in psychiatric outpatients. $J$ Pers Assess, 67, 588-597.

Beck, DA \& Koenig, HG (1996). Minor depression: a review in literature. Int J Psychiatry Med, 
26, 177-209.

Beevers, CG, Keitner, GI, Ryan, CE \& Miller, IW (2003). Cognitive predictors of symptom return following depression treatment. J Abnorm Psychol, 112, 488-496.

Berg, IA \& Rapaport, GM (1954). Response bias in an unstructured questionnaire. The journal of Psychology, 38, 475-481.

Bialik, RJ, Ravindran, AV, Bakish, D \& Lapierre, YD (1995). A comparison of placebo responders and nonresponders in subgroups of depressive disorder. J Psychiatry Neurosci, 20, 265-270.

Bijl, RV \& Ravelli, A (2000). Current and residual functional disability associated with psychopathology: findings from the Netherlands Mental Health Survey and Incidence Study (NEMESIS). Psychol Med, 30, 657-668.

Bijl, RV, Ravelli, A \& van Zessen, G (1998). Prevalence of psychiatric disorder in the general population: results of the Netherlands Mental Health Survey and Incidence Study (NEMESIS). Soc Psychiatry Psychiatr Epidemiol, 33, 587-595.

Blazer, DG, Kessler, RC, McGonagle, KA \& Swartz, MS (1994). The prevalence and distribution of major depression in a national community sample: the National Comorbidity Survey. $\mathrm{Am}$ $J$ Psychiatry, 151, 979-986.

Bower, P \& Gilbody, S (2005). Stepped care in psychological therapies: access, effectiveness and efficiency. Br J Psychiatry, 186, 11-17.

Bower, P, Rowland, N \& Hardy, R (2003). The clinical effectiveness of counselling in primary care: a systematic review and meta-analysis. Psychol Med, 33, 203-215.

Brazier, J, Roberts, J \& Deverill, M (2002). The estimation of a preference-based measure of health from the SF-36. $J$ Health Econ, 21, 271-292.

Brazier, JE \& Roberts, J (2004). The estimation of a preference-based measure of health from the SF-12. Med Care, 42, 851-859.

Briggs, AH (2001). Handling uncertainty in economic evaluation and presenting results. In Economic evaluation in health care; merging theory with practice (eds M Drummond \& A McGuire). Oxford: Oxford University Press.

Briggs, AH, Wonderling, DE \& Mooney, CZ (1997). Pulling cost-effectiveness analysis up by its bootstraps: a non-parametric approach to confidence interval estimation. Health Econ, 6 , 405-415.

Brooks, R (1996). EuroQol: the current state of play. Health Policy, 37, 53-72.

Brugha, TS \& Bebbington, PE (1992). The undertreatment of depression. Eur Arch Psychiatry Clin Neurosci, 242, 103-108.

Brunenberg, DE, van Steyn, MJ, Sluimer, JC, Bekebrede, LL, Bulstra, SK \& Joore, MA (2005). Joint recovery programme versus usual care. An economic evaluation of a clinical pathway for joint replacement surgery. Med Care, 43, 1018-1026.

Buchanan, T (2003). Internet-based questionnaire assessment: appropriate use in clinical contexts. Cognitive Behaviour Therapy, 32, 100-109.

Butcher, JN, Perry, J \& Hahn, J (2004). Computers in clinical assessment: historical developments, present status, and future challenges. J Clin Psychol, 60, 331-345.

Butcher, JN, Perry, JN \& Atlis, MM (2000). Validity and utility of computer-based test interpretation. Psychological Assessment, 12, 6-18.

Butler, AC, Chapman, JE, Forman, EM \& Beck, AT (2006). The empirical status of cognitivebehavioral therapy: a review of meta-analyses. Clin Psychol Rev, 26, 17-31.

Cane, DB, Olinger, J, Gotlib, IH \& Kuiper, NA (1986). Factor structure of the dysfunctional attitude scale in a student population. $J$ Clin Psychol, 42, 307-309.

Carlbring, P \& Andersson, G (2006). Internet and psychological treatment. How well can they be combined? Computers in Human Behavior, 22, 545-553.

Carlbring, P, Brunt, S, Bohman, S, Austin, D, Richards, J, Öst, LG \& Andersson, G (2007a). Internet vs paper and pencil administration of questionnaires commonly used in panic/ agoraphobia research. Computers in Human Behavior, 23, 1421-1434.

Carlbring, P, Gunnarsdóttir, M, Hedensjö, L, Andersson, G, Ekselius, L \& Furmark, T (2007b). Treatment of social phobia: randomised trial of Internet-delivered cognitive-behavioural therapy with telephone support. Br J Psychiatry, 190, 123-128.

Carr, AJ, Gibson, B \& Robinson, PG (2001). Is quality of life determined by expectations or experience? Br Med J, 322, 1240-1243. 
Casparie, AF, van hout, BA \& Simoons, ML (1998). Richtlijnen en kosten. Ned Tijdschr Geneeskd, 142, 2075-2077.

Cavanagh, K \& Shapiro, DA (2004). Computer treatment for common mental health problems. J Clin Psychol, 60, 239-251.

CBO (2005). Multidisciplinaire richtlijn depressie: richtlijn voor de diagnostiek en behandeling van volwassen cliënten met een depressie. Utrecht: Trimbos-instituut.

Chalmers, I (1985). Proposal to outlaw the term "negative trial". Br Med J (Clin Res Ed), 290, 1002.

Chambless, DL (2002). Beware the Dodo bird: the dangers of overgeneralization. Clinical Psychology: Science and Practice, 9, 13-16.

Chen, LS, Eaton, WW, Gallo, JJ, Nestadt, G \& Crum, RM (2000). Empirical examination of current depression categories in a population-based study: symptoms, course, and risk factors. Am J Psychiatry, 157, 573-580.

Chioqueta, AP \& Stiles, TC (2006). Factor structure of the Dysfunctional Attitude Scale (Form A) and the Automatic Thoughts Questionnaire: an exploratory study. Psychol Rep, 99, 239-247.

Christensen, H, Griffiths, K, Groves, C \& Korten, A (2006a). Free range users and one hit wonders: community users of an Internet-based cognitive behaviour therapy program. Aust $N$ Z J Psychiatry, 40, 59-62.

Christensen, H \& Griffiths, KM (2002). The prevention of depression using the Internet. Med J Aust, 177, S122-S125.

Christensen, H, Griffiths, KM \& Jorm, AF (2004a). Delivering interventions for depression by using the Internet: randomised controlled trial. $\mathrm{Br} \mathrm{Med} \mathrm{J,} \mathrm{328,} 7434$.

Christensen, H, Griffiths, KM, Korten, AE, Brittliffe, K \& Groves, C (2004b). A comparison of changes in anxiety and depression symptoms of spontaneous users and trial participants of a cognitive behaviour therapy website. $J$ Med Internet Res, 6, e46.

Christensen, H, Griffiths, KM, Mackinnon, AJ \& Brittliffe, K (2006b). Online randomized controlled trial of brief and full cognitive behaviour therapy for depression. Psychol Med, 36, 17371746.

Clark, DA, Beck, AT \& Alford, BA (1999). Scientific foundations of cognitive theory and therapy of depression. New York: Wiley.

Clarke, G, Eubanks, D, O'Connor, E, DeBar, LL, Kelleher, C, Lynch, F \& Nunley, S (2002). Overcoming depression on the Internet (ODIN): a randomised controlled trial of an Internet depression skills intervention program. $J$ Med Internet Res, 4, e14.

Clarke, G, Eubanks, D, Reid, E, Kelleher, C, O'Connor, E, DeBar, LL, Lynch, F, Nunley, S \& Gullion, C (2005). Overcoming depression on the Internet (ODIN) (2): a randomized trial of a selfhelp depression skills program with reminders. $J$ Med Internet Res, 7, e16.

Cohen, J (1988). Statistical power analysis for the behavioral sciences. Hillsdale, NJ: Erlbaum.

Cohen, J (1992). Quantitative methods in psychology: a power primer. Psychol Bull, 112, 155159.

College voor Zorgverzekeringen (2007). Farmacotherapeutisch Kompas 2008 [ Pharmacotherapeutic Compass 2008]. Diemen: College voor Zorgverzekeringen.

Comrey, AL \& Lee, HB (1992). A first course in factor analysis. Hillsdale, NJ: Erlbaum.

Conoscenti, LM \& Litz, BT (2008). Book forum. Hands-on help: computer-aided psychotherapy. Am J Psychiatry, 165, 142-143.

Cook, RJ \& Sackett, DL (1995). The number needed to treat: a clinically useful measure of treatment effect. Br Med J, 310, 452-454.

Coyne, JC \& Whiffen, VE (1995). Issues in personality as diathesis for depression: the case of sociotropy-dependency and autonomy-self-criticism. Psychol Bull, 118, 358-378.

Cuijpers, P (1997). Bibliotherapy in unipolar depression: a meta-analysis. J Behav Ther Exp Psychiatry, 28, 139-147.

Cuijpers, P, Bonarius, M \& van den Heuvel, A (1995). De omgaan met depressie cursus: een handreiking voor begeleiders en organisatoren [The coping with depression course: a manual]. Utrecht: NcGv.

Cuijpers, P, de Graaf, R \& van Dorsselaer, S (2004). Minor depression: risk profiles, functional disability, health care use and risk of developing major depression. $J$ Affect Disord, 79, 71-79. 
Cuijpers, P \& Smit, F (2004). Subthreshold depression as a risk indicator for major depressive disorder: a systematic review of prospective studies. Acta Psychiatr Scand, 109, 325331.

Cuijpers, P, Smit, F, Oostenbrink, J, de Graaf, R, ten Have, M \& Beekmand, A (2007a). Economic costs of minor depression: a population-based study. Acta Psychiatr Scand, 115, 229236.

Cuijpers, P, van Straten, A, Andersson, G \& van Oppen, P (2008). Psychotherapy for depression in adults: a meta-analysis of comparative outcome studies. $J$ Consult Clin Psychol, 76, 909-922.

Cuijpers, P, van Straten, A \& Warmerdam, L (2007b). Behavioral activation treatments of depression: a meta-analysis. Clin Psychol Rev, 27, 318-326.

de Graaf, LE, Gerhards, SAH, Arntz, A, Riper, H, Metsemakers, JFM, Evers, SMAA, Severens, JL, Widdershoven, G \& Huibers, MJH (in press-a). Clinical effectiveness of online computerized cognitive behavioural therapy without support for depression in primary care: a randomised trial. Br J Psychiatry.

de Graaf, LE, Gerhards, SAH, Evers, SMAA, Arntz, A, Riper, H, Severens, JL, Widdershoven, G, Metsemakers, JFM \& Huibers, MJH (2008a). Clinical and cost-effectiveness of computerised cognitive behavioural therapy for depression in primary care: design of a randomised trial. BMC Public Health, 8, 224.

de Graaf, LE, Huibers, MJH, Cuijpers, P \& Arntz, A (2008b). Minor and major depression: does dysfunctional thinking play a role? Manuscript submitted for publication.

de Graaf, LE, Roelofs, J \& Huibers, MJH (in press-b). Measuring dysfunctional attitudes in the general population: the Dysfunctional Attitude Scale (form A) revised. Cognitive Therapy and Research.

de Graaf, R, Bijl, RV, ten Have, M, Beekman, ATF \& Vollebergh, WAM (2004). Rapid onset of comorbidity of common mental disorders: findings from the Netherlands Mental Health Survey and Incidence Study (NEMESIS). Acta Psychiatr Scand, 109, 55-63.

de Jonge, P \& Slaets, JPJ (2005). Response sets in self-report data and their associations with personality traits. European Journal of Psychiatry, 19, 209-214.

Derogatis, LR, Rickels, K \& Rock, AF (1976). The SCL-90 and the MMPI: A step in the validation of a new self-report scale. Br J Psychiatry, 128, 280-289.

DeRubeis, RJ, Hollon, SD, Amsterdam, JD, Shelton, RC, Young, PR, Salomon, RM, O'Reardon, JP, Lovett, ML, Gladdis, MM, Brown, LL \& Gallop, R (2005). Cognitive therapy vs medications in the treatment of moderate to severe depression. Arch Gen Psychiatry, 62, 409416.

Devilly, GJ \& Borkovec, TD (2000). Psychometric properties of the credibility/expectancy questionnaire. J Behav Ther Exp Psychiatry, 31, 73-86.

Dimidjian, S, Hollon, SD, Dobson, KS, Schmaling, KB, Kohlenberg, RJ, Addis, ME, Gallop, R, McGlinchey, JB, Markley, DK, Gollan, JK, Atkins, DC, Dunner, DL \& Jacobson, NS (2006). Randomized trial of behavioral activation, cognitive therapy, and antidepressant medication in the acute treatment of adults with major depression. J Consult Clin Psychol, 74, 657-670.

Dolan, $\mathrm{P}$ (1997). Modeling valuations for EuroQol health states. Med Care, 35, 1095-1108.

Drummond, MF, Sculpher, MJ, Torrance, GW, O'Brien, B \& Stoddart, GL (2005). Methods for the economic evaluation of health care programmes. Oxford: Oxford University Press.

Dyck, MJ (1992). Subscales of the Dysfunctional Attitude Scale. Br J Clin Psychol, 31, 333-335.

Edlund, MJ, Wang, PS, Berglund, PA, Katz, SJ, Lin, E \& Kessler, RC (2002). Dropping out of mental health treatment: patterns and predictors among epidemiological survey respondents in the United States and Ontario. Am J Psychiatry, 159, 845-851.

Elkin, I (1999). A major dilemma in psychotherapy outcome research: disentangling therapists from therapies. Clinical Psychology: Science and Practice, 6, 10-32.

EuroQol Group (1990). EuroQol - a new facility for the measurement of health-related quality of life. Health Policy, 16, 199-208.

Evans, C, Margison, F \& Barkham, M (1998). The contribution of reliable and clinically significant change methods to evidence-based mental health. Evid Based Ment Health, 1, 70-72.

Eysenbach, G (2005). The law of attrition. J Med Internet Res, 7, e11.

Farvolden, P, Denisoff, E, Selby, P, Bagby, RM \& Rudy, L (2005). Usage and longitudinal effective- 
ness of a web-based self-help cognitive behavioral therapy program for panic disorder. $J$ Med Internet Res, 7, e7.

Fenwick, E, Claxton, K \& Sculpher, M (2001). Representing uncertainty: the role of cost-effectiveness acceptibility curves. Health Econ, 10, 779-787.

Fenwick, E, O'Brien, BJ \& Briggs, A (2004). Economic evaluation. Cost-effectiveness acceptability curves - facts, fallacies and frequently asked questions. Health Econ, 13, 405-415.

Field, A (2000). Discovering statistics using SPSS for Windows. London: Sage publications Ltd.

Flett, GL, Vredenburg, K \& Krames, L (1997). The continuity of depression in clinical and nonclinical samples. Psychol Bull, 121, 395-416.

Floyd, M, Scogin, F \& Chaplin, WF (2004). The Dysfunctional Attitude Scale: factor structure, reliability and validity with older adults. Aging Ment Health, 8, 153-160.

Fogel, J, Eaton, WW \& Ford, DE (2006). Minor depression as a predictor of the first onset of major depressive disorder over a 15-year follow-up. Acta Psychiatr Scand, 113, 36-43.

Fournier, JC, DeRubeis, RJ, Shelton, RC, Gallop, R, Amsterdam, J \& Hollon, SD (2008). Antidepressant medications $\mathrm{v}$. cognitive therapy in people with depression with or without personality disorders. Br J Psychiatry, 192, 124-129.

Friedman, MA, Detweiler-Bedell, JB, Leventhal, HE, Horne, R, Keitner, GI \& Miller, IW (2004). Combined psychotherapy and pharmacotherapy for the treatment of major depressive disorder. Clinical Psychology: Science and Practice, 11, 47-68.

Galbaud du Fort, G, Newman, SC, Boothroyd, LJ \& Bland, C (1999). Treatment seeking for depression: role of depressive symptoms and comorbid psychiatric diagnoses. J Affect Disord, 52, 31-40.

Garb, HN (2007). Computer-administered interviews and rating scales. Psychological Assessment, 19, 4-13.

Gega, L, Kenwright, M, Mataix-Cols, D, Cameron, R \& Marks, IM (2005). Screening people with anxiety/depression for suitability for guided self-help. Cognitive Behaviour Therapy, 34, 16-21.

Gega, L, Marks, I \& Mataix-Cols, D (2004). Computer-aided CBT self-help for anxiety and depressive disorders: experience of a London clinic and future directions. Journal of Clinical Psychology/In Session, 60, 147-157.

Gibb, BE, Alloy, LB, Abramson, LY, Beevers, CG \& Miller, IW (2004). Cognitive vulnerability to depression: a taxometric analysis. J Abnorm Psychol, 113, 81-89.

Gloaguen, V, Cottraux, J, Cucherat, M \& Blackburn, IM (1998). A meta-analysis of the effects of cognitive therapy in depressed patients. $J$ Affect Disord, 49, 59-72.

Goossens, ME, Vlaeyen, JW, Hidding, A, Kole-Snijders, A \& Evers, SM (2005). Treatment expectancy affects the outcome of cognitive-behavioral interventions in chronic pain. The Clinical Journal of Pain, 21, 18-26.

Goossens, MEJB, Rutten-van Mölken, MPMH, Vlaeyen, JWS \& van der Linden, SMJP (2000). The cost-diary: a method to measure direct and indirect costs in cost effectiveness research. J Clin Epidemiol, 53, 688-695.

Greenberg, RP, Constantino, MJ \& Bruce, N (2006). Are patient expectations still relevant for psychotherapy process and outcome? Clin Psychol Rev, 26, 657-678.

Griffiths, K, Farrer, L \& Christensen, H (2007). Clickety-click: e-mental health train on track. Australasian Psychiatry, 15, 100-108.

Gyrd-Hansen, D (2003). Willingness to pay for a QALY. Health Econ, 12, 1049-1060.

Hakkaart-van Roijen, L (2002). Trimbos/iMTA questionnaire for costs associated with psychiatric illness (TiC-P). Rotterdam: Erasmus University.

Hamilton, KE \& Dobson, KS (2002). Cognitive therapy of depression: pretreatment patient predictors of outcome. Clin Psychol Rev, 22, 875-893.

Hedluns, JL, Vieweg, BW \& Cho, DW (1985). Mental health computing in the 1980s: II. Clinical applications. Computers in Human Services, 1, 1-31.

Hegel, MT, Oxman, TE, Hull, JG, Swain, K \& Swick, H (2006). Watchful waiting for minor depression in primary care: remission rates and predictors of improvement. Gen Hosp Psychiatry, 28, 205-212.

Hill, CV, Oei, TPS \& Hill, MA (1989). An empirical investigation of the specificity and sensitivity of the Automatic Thoughts Questionnaire and Dysfunctional Attitudes Scale. Journal of Psychopathology and Behavioral Assessment, 11, 291-311. 
Hirschfeld, RM, Keller, MB, Panico, S, Arons, BS, Barlow, D, Davidoff, F, Ednicott, J, Froom, J, Goldstein, M, Gorman, JM, Marek, RG, Maurer, TA, Meyer, R, Philips, K, Ross, J, Schwenk, TL, Sharfstein, SS, Thase, ME \& Wyatt, RJ (1997). The National Depressive and Manic-Depressive Association consensus statement on the undertreatment of depression. JAMA, 277, 333-340.

Hoeymans, N, van Lindert, H \& Westert, GP (2005). The health status of the Dutch population as assessed by the EQ-6D. Qual Life Res, 14, 655-663.

Hollon, SD, Kendall, PS \& Lumry, A (1986). Specificity of depressotypic cognitions in clinical depression. J Abnorm Psychol, 95, 52-59.

Hollon, SD, Stewart, MO \& Strunk, D (2006). Enduring effects for cognitive behavior therapy in the treatment of depression and anxiety. Annu Rev Psychol, 57, 285-315.

Hopko, DR, Lejuez, CW, Ruggiero, KJ \& Eifert, GH (2003). Contemporary behavioral activation treatments for depression: procedures, principles, and progress. Clin Psychol Rev, 23, 699-717.

llardi, SS \& Craighead, WE (1994). The role of nonspecific factors in cognitive-behavior therapy for depression. Clinical Psychology: Science and Practice, 1, 183-155.

Imber, SD, Pilkonis, PA, Sotsky, SM, Elkin, I, Watkins, JT, Collins, JF, Shea, MT, Leber, WR \& Glass, DR (1990). Mode-specific effects among three treatments for depression. J Consult Clin Psychol, 58, 352-359.

Ingram, RE, Miranda, J \& Segal, ZV (1998). Cognitive vulnerability to depression. New York: Guilford Press.

Jacobson, NS, Martell, CR \& Dimidjian, S (2001). Behavioral activation treatment for depression: returning to contextual roots. Clinical Psychology: Science and Practice, 8, 255-270.

Jacobson, NS \& Truax, $P$ (1991). Clinical significance: a statistical approach to defining meaningful change in psychotherapy research. $J$ Consult Clin Psychol, 59, 12-19.

Jarrett, RB, Eaves, GG, Granneman, BD \& Rush, AJ (1991). Clinical, cognitive, and demographic predictors of response to cognitive therapy for depression: a preliminary report. Psychiatry Res, 37, 245-260.

Jöreskog, KG \& Sörbom, D (1999). LISREL 8.30. Chicago: Scientific Software International.

Jorm, AF, Griffiths, KM, Christensen, H, Parslow, RA \& Rogers, B (2004). Actions taken to cope with depression at different levels of severity: a community sample. Psychol Med, 34, 293-299.

Judd, LL (1997). The clinical course of unipolar major depressive disorders. Arch Gen Psychiatry, 54, 989-991.

Judd, LL, Paulus, MJ, Schettler, PJ, Akiskal, HS, Endicott, J, Leon, AC, Maser, JD, Mueller, T, Solomon, DA \& Keller, MB (2000). Does incomplete recovery from first lifetime major depressive episodes herald a chronic course of illness? Am J Psychiatry, 157, 1501-1504.

Kaltenthaler, E, Brazier, J, De Nigris, E, Tumur, I, Ferriter, M, Beverly, C, Parry, G, Rooney, G \& Sutcliffe, P (2006). Computerised cognitive behaviour therapy for depression and anxiety update: a systematic review and economic evaluation. Health Technol Assess, 10, 1-186.

Kaltenthaler, E, Parry, G, Beverley, C \& Ferriter, M (2008a). Computerised cognitive-behavioural therapy for depression: systematic review. Br J Psychiatry, 193, 181-184.

Kaltenthaler, E, Sutcliffe, P, Parry, G, Beverly, C, Rees, A \& Ferriter, M (2008b). The acceptability to patients of computerized cognitive behaviour therapy for depression: a systematic review. Psychol Med, 38, 1521-1530.

Katon, W, Von Korff, M, Lin, E, Walker, E, Simon, GE, Bush, T, Robinson, P \& Russo, J (1995). Collaborative management to achieve treatment guidelines. Impact on depression in primary care. JAMA, 273, 1026-1031.

Kazantzis, N, Deane, FP \& Ronan, KR (2000). Homework assignments in cognitive and behavioral therapy: a meta-analysis. Clinical Psychology: Science and Practice, 7, 189-202.

Kazdin, AE (2007). Mediators and mechanisms of change in psychotherapy research. Annu Rev Clin Psychol, 3, 1-27.

Keller, MB \& Boland, RJ (1998). Implications of failing to achieve successful long-term maintenance treatment of recurrent unipolar major depression. Biol Psychiatry, 44, 348-360.

Kelloway, EK (1998). Using LISREL for structural equation modeling: a researcher's guide. Thousand Oaks: Sage Publications.

Kenwright, M, Marks, I, Graham, C, Franses, A \& Mataix-Cols, D (2005). Brief scheduled phone 
support from a clinician to enhance computer-aided self-help for obsessive-compulsive disorder: randomized controlled trial. J Clin Psychol, 61, 1499-1508.

Kessler, RC (1997). The effects of stressful life events on depression. Annu Rev Psychol, 48, 191-214.

Kessler, RC, Berglund, P, Demler, O, Jin, R, Koretz, D, Merikangas, KR, Rush, AJ, Walters, EE \& Wang, PS (2003). The epidemiology of major depressive disorder. Results from the National Comorbidity Survey Replication (NCS-R). JAMA, 289, 3095-3105.

Kessler, RC, Zhao, S, Blazer, DG \& Swartz, M (1997). Prevalence, correlates, and course of minor depression and major depression in the national comorbidity survey. $J$ Affect Disord, 45, 19-30.

King, JT, Tsevat, J, Lave, JR \& Roberts, MS (2005). Willingness to pay for a quality-adjusted life yeat: implications for societal health care resource allocation. Med Decis Making, 25, 667-677.

Kirsch, I, Deacon, BJ, Huedo-Medina, TB, Scoboria, A, Moore, TJ \& Johnson, BT (2008). Initial severity and antidepressant benefits: a meta-analysis of data submitted to the food and drug administration. PloS Medicine, 5, e45.

Koeter, MWJ, Ormel, J \& van den Brink, W (1988). Instrumenteel onderzoek: totaalscore op de SCL-90 als maat voor de ernst van psychopathologie. Nederlands Tijdschrift voor de Psychologie, 43, 381-391.

Koopmanschap, M, Meerding, WJ, Evers, S, Severens, J, Burdorf, A \& Brouwer, W (2004). Handleiding voor het gebruik van PRODISQ versie 2.1. Rotterdam/Maastricht: Erasmus MC/ Universiteit Maastricht.

Koopmanschap, MA (2005). PRODISQ: a modular questionnaire on productivity and disease for economic evaluation studies Expert Review of Pharmacoeconomics and Outcomes Research, 5, 23-28.

Koopmanschap, MA, Rutten, FFH, van Ineveld, BM \& van Roijen, L (1995). The friction cost method for estimating the indirect costs of disease. $J$ Health Econ, 14, 171-189.

Krabbe, PFM, Stouthard, MEA, Essink-Bot, M-L \& Bonsel, GJ (1999). The effect of adding a cognitive dimension to the EuroQol multiattribute health-status classification system. J Clin Epidemiol, 52, 293-301.

Kraemer, HC, Wilson, GT, Fairburn, CG \& Agras, WS (2002). Mediators and moderators of treatment effects in randomized clinical trials. Arch Gen Psychiatry, 59, 877-883.

Kruijshaar, ME, Hoeymans, N, Bijl, RV, Spijker, J \& Essink-Bot, ML (2003). Levels of disability in major depression. Findings from the Netherlands Mental Health Survey and Incidence Study (NEMESIS). J Affect Disord, 77, 53-64.

Kwon, S \& Oei, TPS (1992). Differential causal roles of dysfunctional attitudes and automatic thoughts in depression. Cognitive Therapy and Research, 16, 309-328.

Lamers, LM, McDonnell, J, Stalmeier, PFM, Krabbe, PFM \& Busschbach, JJV (2006). The Dutch tariff: results and arguments for an effective design for national EQ-5D valuation studies. Health Econ, 15, 1121-1132.

Lange, A, Vermeulen, H, Renckens, CH, Schrijver, M, van de Ven, JP, Schrieken, B \& Dekker, $J$ (2005). De geprotocolleerde Interapy-behandeling van depressie via het internet; resultaten van een gerandomiseerde trial. Directieve Therapie, 1, 27-50.

Lau, MA, Segal, ZV \& Williams, JMG (2004). Teasdale's differential activation hypothesis: implications for mechanisms of depressive relapse and suicidal behaviour. Behav Res Ther, 42, 1001-1017.

Laupacis, A, Feeny, D, Detsky, AS \& Tugwell, PX (1992). How attractive does a new technology have to be to warrant adoption and utilization? Tentative guidelines for using clinical and economic evaluations. Can Med Assoc J, 146, 473-481.

Lewinsohn, PM, Antonuccio, DO, Steinmetz, JL \& Teri, L (1984). The coping with depression course: a psychoeducational intervention for unipolar depression. Eugene: Castalia Publishing.

Luborsky, L, Rosenthal, R, Diguer, L, Andrusyna, TP, Berman, JS, Levitt, JT, Seligman, DA \& Krause, ED (2002). The Dodo bird verdict is alive and well - mostly. Clinical Psychology: Science and Practice, 9, 2-12.

Luppa, M, Heinrich, S, Angermeyer, MC, König, HH \& Riedel-Heller, SG (2007). Cost-of-illness studies of depression. A systematic review. J Affect Disord, 98, 29-43. 
Mackinnon, AJ, Griffiths, KM \& Christensen, H (2008). Comparative randomised trial of online cognitive-behavioural therapy and an information website for depression: 12-month outcomes. Br J Psychiatry, 192, 130-134.

Manca, A, Hawkins, N \& Sculpher, MJ (2005). Estimating mean QALYs in trial-based cost-effectiveness analysis: the importance of controlling for baseline utility. Health Econ, 14, 487-496.

Manwaring, JL, Bryson, SW, Goldschmidt, AB, Winzelberg, AJ, Luce, KH, Cunning, D, Wilfey, DE \& Taylor, $\mathrm{CB}$ (2008). Do adherence variables predict outcome in an online program for the prevention of eating disorders? J Consult Clin Psychol, 76, 341-346.

Marie-Mitchell, A, Leuchter, AF, Chou, CP, Gauderman, WJ \& Azen, SP (2004). Predictors of improved mood over time in clinical trials for major depression. Psychiatry Res, 127, 73-84.

Marks, I, Shaw, S \& Parkin, R (1998a). Computer-aided treatments of mental health problems. Clinical Psychology: Science and Practice, 5, 151-170.

Marks, IM, Baer, L, Greist, JH, Park, JM, Bachofen, M, Nakagawa, A, Wenzel, KW, Parkin, JR, Manzo, PA, Dottl, SL \& Mantle, JM (1998b). Home self-assessment of obsessive-compulsive disorder. Use of a manual and a computer-conducted telephone interview: two UK-US studies. Br J Psychiatry, 172, 406-412.

Marks, IM, Cavanagh, K \& Gega, L (2007). Computer-aided psychotherapy: revolution or bubble? Br J Psychiatry, 191, 471-473.

Marks, IM, Mataix-Cols, D, Kenwright, M, Cameron, R, Hirsch, S \& Gega, L (2003). Pragmatic evaluation of computer-aided self-help for anxiety and depression. $\mathrm{Br} J$ Psychiatry, 183, 57-65.

Marsh, HW, Balla, JR \& McDonals, RP (1988). Goodness-of-fit indexes in confirmatory factor analysis: the effect of sample size. Psychol Bull, 103, 391-410.

Marsh, HW, Hau, KT, Balla, JR \& Grayson, D (1998). Is more ever too much? The number of indicators per factor in confirmatory factor analysis. Multivariate Behavioral Research, 33, 181-220.

Mazure, CM, Raghaven, C, Maciejewski, PK, Jacobs, SC \& Bruce, ML (2001). Cognitive-personality characteristics as direct predictors of unipolar major depression. Cognitive Therapy and Research, 25, 215-225.

McCrone, P, Knapp, M, Proudfoot, J, Ryden, C, Cavanagh, K, Shapiro, DA, Ilson, S, Gray, JA, Goldberg, D, Mann, A, Marks, I, Everitt, B \& Tylee, A (2004). Cost-effectiveness of computerised cognitive-behavioural therapy for anxiety and depression in primary care: randomised controlled trial. Br J Psychiatry, 185, 55-62.

McHorney, CA, Ware, JE \& Raczek, AE (1993). The MOS 36-item short form health survey (SF36): II. Psychometric and clinical tests of validity in measuring physical and mental health constructs. Med Care, 31, 247-263.

McKendree-Smith, NL, Floyd, M \& Scogin, FR (2003). Self-administered treatments for depression: a review. J Clin Psychol, 59, 275-288.

Meade, AW \& Bauer, DJ (2007). Power and precision in confirmatory factor analytic tests of measurement invariance. Structural Equation Modeling: A Multidisciplinary Journal, 14, 611-635.

Meehl, PE (1995). Bootstraps taxometrics. Solving the classification problem in psychopathology. Am Psychol, 50, 266-275.

Meyer, B, Pilkonis, PA, Krupnick, JL, Egan, MK, Simmens, SJ \& Sotsky, SM (2002). Treatment expectancies, patient alliance, and outcome: further analyses from the National Institute of Mental Health Treatment of Depression Collaborative Research Program. J Consult Clin Psychol, 70, 1051-1055.

Miranda, J, Persons, JB \& Byers, CN (1990). Endorsement of dysfunctional beliefs depends on current mood state. J Abnorm Psychol, 99, 237-241.

Mitchell, N \& Dunn, K (2007). Pragmatic evaluation of the viability of CCBT self-help for depression in higher education. Counseling and Psychotherapy Research, 7, 144-150.

Mitchell, N \& Gordon, PK (2007). Attitudes towards computerized CBT for depression amongst a student population. Behavioural and Cognitive Psychotherapy, 35, 421-430.

Mulder, RT, Joyce, PR, Frampton, CMA, Luty, SE \& Sullivan, PF (2006). Six months of treatment for depression: outcome and predictors of the course of illness. Am J Psychiatry, 163, 95-100. 
Mundt, JC, Marks, IM, Shear, MK \& Greist, JH (2002). The Work and Social Adjustment Scale: a simple measure of impairment in functioning. Br J Psychiatry, 180, 461-464.

Murphy, R, Cooper, Z, Hollon, SD \& Fairburn, CG (2009). How do psychological treatments work? Investigating mediators of change. Behav Res Ther, 47, 1-5.

Murray, CL \& Lopez, AD (1997). Global mortality, disability, and the contribution of risk factors: Global Burden of Disease Study. Lancet, 349, 1436-1442.

Murray, K, Pombo-Carril, MG, Bara-Carril, N, Grover, M, Reid, Y, Langham, C, Birchall, H, Williams, C, Treasure, J \& Schmidt, U (2003). Factors determining uptake of a CD-ROMbased CBT self-help treatment for bulimia: patient characteristics and subjective appraisals. European Eating Disorders Review, 11, 243-260.

Nederlands Huisartsen Genootschap [Dutch College of General Practitioners] (2003). NHGStandaard Depressieve stoornis (depressie). Utrecht.

Nes, LS \& Segerstrom, SC (2006). Dispositional optimism and coping: a meta-analytic review. Personality and Social Psychology Review, 10, 235.

Newman, MG, Consoli, AJ \& Barr Taylor, C (1999). A palmtop computer program for the treatment of generalized anxiety disorders. Behav Modif, 23, 597-619.

NICE (2006). Computerised cognitive behaviour therapy for depression and anxiety. Review of Technology Appraisal 51. London: National Institute for Health and Clinical Excellence.

Olinger, LJ, Kuiper, NA \& Shaw, BF (1987). Dysfunctional attitudes and stressful life events: an interactive model of depression. Cognitive Therapy and Research, 11, 25-40.

Oliver, JM \& Baumgart, EP (1985). The Dysfunctional Attitude Scale: psychometric properties and relation to depression in an unselected adult population. Cognitive Therapy and Research, 9, 161-167.

Oostenbrink, JB, Bouwmans, CAM, Koopmanschap, MA \& Rutten, FFH (2004). Handleiding voor kostenonderzoek: methoden en standaard kostprijzen voor economische evaluaties in de gezondheidszorg (geactualiseerde versie 2004). Diemen: College voor Zorgverzekeringen.

Oostenbrink, JB, Koopmanschap, MA \& Rutten, FFH (2002). Standardisation of costs. The Dutch manual for costing in economic evaluations. Pharmacoeconomics, 20, 443-454.

Orley, JJ, Saxena, S \& Herman, H (1998). Quality of life and mental illness. Reflections from the perspective of WHOQOL. Br J Psychiatry, 172, 291-293.

Osgood-Hynes, DJ, Greist, JH, Marks, IM, Baer, L, Heneman, SW, Wenzel, KW, Manzo, PA, Parkin, JR, Spierings, CJ, Dottl, SL \& Vitse, HM (1998). Self-administered psychotherapy for depression using a telephone-accessed computer system plus booklets: an open U.S.U.K. study. J Clin Psychiatry, 59, 358-365.

Otto, MW, Smits, JAJ \& Reese, HE (2005). Combined psychotherapy and pharmacotherapy for mood and anxiety disorders in adults: review and analysis. Clinical Psychology: Science and Practice, 23, 72-86.

Pampallona, S, Bollini, P, Tibaldi, G, Kupelnick, B \& Munizza, C (2004). Combined pharmacotherapy and psychological treatment for depression. Arch Gen Psychiatry, 61, 714-719.

Parker, G, Bradshaw, G \& Blignault, I (1984). Dysfunctional attitudes: measurement, significant constructs and links with depression. Acta Psychiatr Scand, 70, 90-96.

Patten, SB (2003). Prevention of depressive symptoms through the use of distance technologies. Psychiatr Serv, 54, 396-398.

Paykel, ES, Scott, J, Cornwall, PE, Abbott, R, Crane, C, Pope, M \& Johnson, AL (2005). Duration of relapse prevention after cognitive therapy in residual depression: follow-up of controlled trial. Psychol Med, 35, 59-68.

Paykel, ES, Tylee, A, Wright, A \& Priest, RG (1997). The Defeat Depression Campaign: psychiatry in the public arena. Am J Psychiatry, 154, 59-65.

Persons, JB, Burns, DD \& Perloff, JM (1988). Predictors of dropout and outcome in cognitive therapy for depression in a private practice. Cognitive Therapy and Research, 12, 557575.

Petersen, TJ, Feldman, G, Harley, R, Fresco, DM, Graves, L, Holmes, A, Bogdan, R, Papakostas, GI, Bohn, L, Lury, RA, Fava, M \& Segal, ZV (2007). Extreme response style in recurrent and chronically depressed patients: change with antidepressant administration and stability during continuation treatment. J Consult Clin Psychol, 75, 145-153.

Peterson, L, Johansson, V \& Carlsson, SG (1996). Computerised testing in a hospital setting: 
psychometric and psychological effects. Computers in Human Behaviour, 12, 339-350.

Pincus, AL \& Gurtman, MB (1995). The three faces of interpersonal dependency: structural analyses of self-report dependency measures. J Pers Soc Psychol, 69, 744-758.

Posternak, MA \& Miller, I (2001). Untreated short-term course of major depression: a meta-analysis of outcomes from studies using wait-list control groups. J Affect Disord, 66, 139-146.

Power, MJ, Duggan, CF, Lee, AS \& Murrey, RM (1995). Dysfunctional attitudes in depressed and recovered depressed patients and their first-degree relatives. Psychol Med, 25, 87-93.

Power, MJ, Katz, R, McGuffin, P, Duggan, CF, Lam, D \& Beck, AT (1994). The Dysfunctional Attitude Scale (DAS). A comparison of forms $A$ and $B$ and proposals for a new subscaled version. Journal of Research in Personality, 28, 263-276.

Prisciandaro, JJ \& Roberts, JE (2005). A taxometric investigation of unipolar depression in the national comorbidity survey. J Abnorm Psychol, 114, 718-728.

Proudfoot, J, Goldberg, A, Mann, A, Everitt, B, Marks, I \& Gray, JA (2003). Computerized, interactive, multimedia cognitive behavioral program for anxiety and depression in general practice. Psychol Med, 33, 217-227.

Proudfoot, J, Ryden, C, Everitt, B, Shapiro, DA, Goldberg, A, Mann, A, Tylee, A, Marks, I \& Gray, JA (2004). Clinical efficacy of computerised cognitive-behavioural therapy for anxiety and depression in primary care: randomised controlled trial. Br J Psychiatry, 185, 46-54.

Raad voor de Volksgezondheid en Zorg [Council for Public Health and Health Care] (2006). Zinnige en duurzame zorg [Sensible and sustainable care]. Zoetermeer: Raad voor de Volksgezondheid en Zorg [Council for Public Health and Health Care].

Raes, F, Hermans, D, van den Broeck, K \& Eelen, P (2005). Kort instrumenteel: de Nederlandstalige versie van de Dysfunctional Attitude Scale-vorm A (DAS-A-NL) [the Dutch version of the Dysfunctional Attitude Scale-form A (DAS-A-NL)]. Gedragstherapie, 38, 285-294.

Renaud, J, Brent, DA, Baugher, M, Birmaher, B, Kolko, DJ \& Bridge, J (1998). Rapid response to psychosocial treatment for adolescent depression: a two-year follow-up. J Am Acad Child Adolesc Psychiatry, 37, 1184-1190.

Riper, H \& Kramer, JJAM (2004). Online zelfhulpcursus www.kleurjeleven.nl [Online selfhelp course www.kleurjeleven.nl ]. Utrecht: Trimbos-institute.

Robins, LN, Wing, J, Ulrich Wittchen, H, Helzer, JE, Babor, TF, Burke, J, Farmer, A, Jablenski, A, Pickens, R, Regier, DA, Sartorius, N \& Towle, LH (1988). The Composite International Diagnostic Interview. Arch Gen Psychiatry, 45, 1069-1077.

Ruscio, J \& Ruscio, AM (2004a). Clarifying boundary issues in psychopathology: the role of taxometrics in a comprehensive program of structural research. J Abnorm Psychol, 113, 24-38.

Ruscio, J \& Ruscio, AM (2004b). A conceptual and methodological checklist for conducting a taxometric investigation. Behavior Therapy, 35, 403-447.

Ruscio, J, Zimmerman, M, McGlinchey, JB, Chelminski, I \& Young, D (2007). Diagnosing major depressive disorder: $\mathrm{XI}$. A taxometric investigation of the categorical-dimensional debate on the structure underlying DSM-IV symptoms. J Nerv Ment Dis, 195, 10-19.

Sahin, NH \& Sahin, N (1992). How dysfunctional are the dysfunctional attitudes in another culture? Br J Med Psychol, 65, 17-26.

Sato, T \& McCann, D (1998). Individual differences in relatedness and individuality: an exploration of two constructs. Personality and individual differences, 24, 847-859.

Schneider, AJ, Mataix-Cols, D, Marks, IM \& Bachofen, M (2005). Internet-guided self-help with or without exposure therapy for phobic and panic disorders: a randomised controlled trial. Psychother Psychosom, 74, 154-164.

Schulenberg, SE \& Yutrzenka, BA (2001). Equivalence of computerized and conventional versions of the Beck Depression Inventory-II (BDI-II). Current Psychology, 20, 216-230.

Scogin, FR, Hanson, A \& Welsh, D (2003). Self-administered treatment in stepped-care models of depression treatment. J Clin Psychol, 59, 341-349.

Segal, ZV, Gemar, M \& Williams, S (1999). Differential cognitive response to a mood challenge following succesful cognitive therapy or pharmacotherapy for unipolar depression. Abnormal Psychology, 108, 3-10.

Seivewright, H, Tyrer, P \& Johnson, T (1998). Prediction of outcome in neurotic disorder: a 5-year prospective study. Psychol Med, 28, 1149-1157.

Selmi, PM, Klein, MH, Greist, JH, Sorrell, SP \& Erdman, HP (1990). Computer-administered cog- 
nitive-behavioral therapy for depression. Am J Psychiatry, 147, 51-56.

Sheeran, T \& Zimmerman, M (2002). Case identification of depression with self-report questionnaires. Psychiatry Res, 109, 51-59.

Sheppard, LC \& Teasdale, JD (2000). Dysfunctional thinking in major depressive disorder: a deficit in metacognitive monitoring. J Abnorm Psychol, 109, 768-776.

Shih, JH (2006). Sex differences in stress generation: an examination of sociotropy/autonomy, stress, and depressive symptoms. Personality and social psychology bulletin, 32, 434446.

Simon, GE, VonKorff, M, Heiligstein, JH, Revicki, DA, Grothaus, L, Katon, W \& Wagner, EH (1996). Initial antidepressant choice in primary care: effectiveness and cost of fluoxetine vs tricyclic antidepressants. JAMA, 275, 1897-1902.

Skinner, BF (1953). Science and human behaviour. New York: Macmillan.

Slobbe, LCJ, Kommer, GJ, Smit, JM, Groen, J, Meerding, WJ \& Polder, JJ (2006). Kosten van ziekten in Nederland 2003; zorg voor euro's - 1. Bilthoven: Rijksinstituut voor Volksgezondheid en Milieu.

Smit, F, Willemse, G, Koopmanschap, M, Onrust, S, Cuijpers, P \& Beekman, A (2006). Costeffectiveness of preventing depression in primary care patients. Br J Psychiatry, 188, 330336.

Sobocki, P, Jönsson, B, Angst, J \& Rehnberg, C (2006). Cost of depression in Europe. J Ment Health Policy Econ, 9, 87-98.

Solomon, A, Haaga, DAF \& Arnow, BA (2001). Is clinical depression distinct from subthreshold depressive symptoms? A review of the continuity issue in depression research. $J$ Nerv Ment Dis, 189, 498-506.

Solomon, A, Ruscio, J, Seeley, JR \& Lewinsohn, PM (2006). A taxometric investigation of unipolar depression in a large community sample. Psychol Med, 36, 1-13.

Spek, V, Cuijpers, P, Nyklícek, I, Riper, H, Keyzer, J \& Pop, V (2007a). Internet-based cognitive behaviour therapy for symptoms of depression and anxiety: a meta-analysis. Psychol Med, 37, 319-328.

Spek, V, Cuijpers, P, Nyklí ek, I, Smits, N, Riper, H, Keyzer, J \& Pop, V (2008a). One-year followup results of a randomized controlled clinical trial on Internet-based cognitive behavioural therapy for subthreshold depression in people over 50-years. Psychol Med, 38, 635-639.

Spek, V, Nyklí ek, I, Cuijpers, P \& Pop, V (2008b). Predictors of outcome of group and Internetbased cognitive behavior therapy. $J$ Affect Disord, 105, 137-145.

Spek, V, Nyklícek, I, Smits, N, Cuijpers, P, Riper, H, Keyzer, J \& Pop, V (2007b). Internet-based cognitive behavioural therapy for subthreshold depression in people over 50 years old: a randomized controlled clinical trial. Psychol Med, 37, 1797-1806.

Spijker, J, Bijl, RV, de Graaf, R \& Nolen, WA (2001). Care utilization and outcome of DSM-III-R major depression in the general population. Results from the Netherlands Mental Health Survey and Incidence Study (NEMESIS). Acta Psychiatr Scand, 104, 19-24.

Statistics Netherlands www.cbs.nl.

Steer, RA, Cavalieri, TA, Leonard, DM \& Beck, AT (1999). Use of the Beck Depression Inventory for Primary Care to screen for major depressive disorders. Gen Hosp Psychiatry, 21, 106111.

Steketee, G \& Chambless, DL (1992). Methodological issues in prediction of treatment outcome. Clin Psychol Rev, 12, 387-400.

Stewart, WF, Ricci, JA, Chee, E, Hanh, SR \& Morganstein, D (2003). Cost of lost productive work time among US workers with depression. JAMA, 289, 3135-3144.

Stinnett, AA \& Mullahy, J (1998). Net health benefits: a new framework for the analysis of uncertainty in cost-effectiveness analysis. Med Decis Making, 18, S68-S80.

Stouthard, MEA, Essink-Bot, ML, Bonel, GJ, Barendregt, JJ, Kramers, PGN, van de Water, HPA, Gunning-Schepers, LJ \& van der Maas, PJ (1997). Disability weights for diseases in the Netherlands. Rotterdam: Department of Public Health, Erasmus University Rotterdam.

Strecher, VJ, Shiffman, S \& West, R (2005). Randomized controlled trial of web-based computertailored smoking cessation program as a supplement to nicotine patch therapy. Addiction, 100, 682-688.

Szende, A, Oppe, M \& Devlin, N (2007). EQ-5D value sets: inventory, comparative review and user guide. Dordrecht: Springer. 
Teasdale, JD (1988). Cognitive vulnerability to persistent depression. Cognition and emotion, 2 , 247-274.

Teasdale, JD, Scott, J, Moore, RG, Hayhurst, H, Pope, M \& Paykel, ES (2001). How does cognitive therapy prevent relapse in residual depression? Evidence from a controlled trial. $J$ Consult Clin Psychol, 69, 347-357.

Teasdale, JD, Segal, ZV, Williams, JMG, Ridgeway, VA, Soulsby, JM \& Lau, MA (2000). Prevention of relapse/recurrence in major depression by mindfulness-based cognitive therapy. $J$ Consult Clin Psychol, 68, 615-623.

Thase, ME, Reynolds, CF, Frank, E, Simons, AD, Garamono, GD, McGeary, J, Harden, T, Fasiczka, AL \& Cahalane, JF (1994). Response to cognitive-behavioral therapy in chronic depression. J Psychother Pract Res, 3, 204-214.

Tiemens, BG, Ormel, J \& Simon, GE (1996). Occurrence, recognition, and outcome of psychological disorders in primary care. Am J Psychiatry, 153, 636-644.

Titov, N (2007). Status of computerized cognitive behavioural therapy for adults. Aust $N Z J$ Psychiatry, 41, 95-114.

Trompenaars, FJ, Masthoff, ED, van Heck, GL, Hodiamont, PP \& de Vries, J (2005). Content validity, construct validity, and reliability of the WHOQOL-Bref in a population of Dutch adult psychiatric outpatients. Qual Life Res, 14.

Vaglum, P \& Falkum, E (1999). Self-criticism, dependency and depressive symptoms in a nationwide sample of Norwegian physicians. J Affect Disord, 52, 153-159.

van Asselt, ADI, Dirksen, CD, Arntz, A, Giesen, J, van Dyck, R, Spinhoven, P, van Tilburg, W, Kremers, I, Nadort, M \& Severens, H (2008). Outpatient psychotherapy for borderline personality disorder: the cost-effectiveness of schema-focused therapy versus transferencefocused psychotherapy. Br J Psychiatry, 192, 450-457.

van Asselt, ADI, van Mastrigt, GAPG, Dirksen, CD, Arntz, A, Severens, JL \& Kessels, AGH (2009). How to deal with cost differences at baseline. Pharmacoeconomics, in press.

van den Brink, M, van den Hout, WB, Stiggelbout, AM, van de Velde, CJH \& Kievit, J (2004). Cost measurement in economic evaluations of health care. Whom to ask? Med Decis Making, 42, 740-746.

van der Does, AJW (2002). De Nederlandse versie van de Beck Depression Inventory - second edition (BDI-II-NL): handleiding. Enschede: The Psychological Corporation.

Van, HL, Schoevers, RA, Kool, S, Hendriksen, M, Peen, J \& Dekker, J (2008). Does early response predict outcome in psychotherapy and combined therapy for major depression? J Affect Disord, 105, 261-265.

van IJzendoorn, MH (1984). Answers without questions: a note on response style questionnaires. Percept Mot Skills, 59, 827-831.

van Marwijk, HWJ, Grundmeijer, HGLM, Bijl, D, van Gelderen, MG, de Haan, M, van Weel-Baumgarten, EM, Burgers, JS, Boukes, FS \& Romeijnders, ACM (2003). NHG-standaard depressieve stoornis. Huisarts Wet, 46, 614-623.

van Schaik, DJF, van Marwijk, HWJ, van der Windt, DAWM, Beekman, ATF, de Haan, M \& van Dyck, $R$ (2002). De effectiviteit van psychotherapie in de eerste lijn bij patiënten met een depressieve stoornis. Tijdschrift voor Psychiatrie, 9, 609-619.

van Straten, A, Tiemens, B, Hakkaart, L, Nolen, WA \& Donker, MCH (2006). Stepped-care vs. matched care for mood and anxiety disorders: a randomized trial in routine practice. Acta Psychiatr Scand, 113, 468-476.

Vittengl, JR, Clark, LA, Dunn, TW \& Jarrett, RB (2007). Reducing relapse and recurrence in unipolar depression: a comparative meta-analysis of cognitive-behavioral therapy's effects. $J$ Consult Clin Psychol, 75, 457-488.

Vuorilehto, M, Melartin, TK, Rytsälä, HJ \& Isometsä, E (2007). Do characteristics of patients with major depressive disorder differ between primary and psychiatric care? Psychol Med, 37, 893-904.

Waller, R \& Gilbody, S (in press). Barriers to the uptake of computerized cognitive behavioural therapy: a systematic review of the quantitative and qualitative evidence. Psychol Med.

Walsh, BT, Seidman, SN, Sysko, R \& Gould, M (2002). Placebo response in studies of major depression: variable, substantial, and growing. JAMA, 287, 1840-1847.

Ware, JE \& Sherbourne, CD (1992). The MOS 36-item short-form health survey (SF-36). I. Conceptual framework and item selection. Med Care, 30, 473-483. 
Warmerdam, L, Van Straten, A, Twisk, J, Riper, H \& Cuijpers, P (2008). Internet-based treatment for adults with depressive symptoms: randomized controlled trial. J Med Internet Res, 10, e44.

Weissman, AN (1979). Assessing depressogenic attitudes: a validation study. Unpublished thesis, University of Pennsylvania.

Weissman, AN \& Beck, AT (1978). Development and validation of the Dysfunctional Attitude Scale; paper presented at the annual meeting of the Association for the Advancement of Behavior Therapy. Chicago.

WHOQOL-Group (1998). Development of the World Health Organization WHOQOL-BREF quality of life assessment. Psychol Med, 28, 551-558.

Wilcox, RR (2005). Introduction to robust estimation and hypothesis testing (2 edn). San Diego: Elsevier Academic Press.

Willemse, GRWM, Smit, F, Cuijpers, P \& Tiemens, BG (2004). Minimal-contact psychotherapy for sub-threshold depression in primary care. Br J Psychiatry, 185, 416-421.

Williams, C \& Whitfield, G (2001). Written and computer-based self-help treatments for depression. Br Med Bull, 57, 133-144.

Winter, LB, Steer, RA, Jones-Hicks, L \& Beck, AT (1999). Screening for major depression disorders in adolescent medical outpatients with the Beck Depression Inventory for Primary Care. J Adolesc Health, 24, 389-394.

Wittchen, HU (1994). Reliability and validity studies of the WHO-Composite International Diagnostic Interview (CIDI): a critical review. Journal of psychiatry research, 28, 57-84.

Wolf, NJ \& Hopko, DR (2008). Psychosocial and pharmacological interventions for depressed adults in primary care: a critical review. Clin Psychol Rev, 28, 131-161.

Wright, JH, Wright, AS, Albano, AM, Basco, MR, Goldsmith, LJ, Raffield, T \& Otto, MW (2005). Computer-assisted cognitive therapy for depression: maintaining efficacy while reducing therapist time. Am J Psychiatry, 162, 1158-1164.

Zimmerman, M, Chelminski, I, McGlinchey, JB \& Young, D (2006). Diagnosing major depressive disorder Vl; performance of an objective test as a diagnostic criterion. J Nerv Ment Dis, 194, 565-569.

Zimmerman, M, Sheeran, T \& Young, D (2004). The Diagnostic Inventory for Depression: a selfreport scale to diagnose DSM-IV major depressive disorder. Clinical Psychology, 60, 87110.

Zwick, WR \& Velicer, WF (1986). Comparison of five rules for determining the number of components to retain. Psychol Bull, 99, 432-442. 


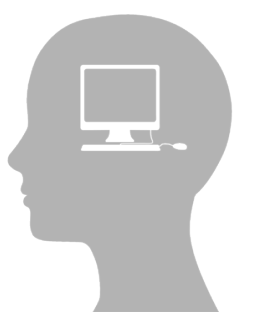

Dankwoord

(Acknowledgements) 

Er zijn veel mensen die op directe of indirecte manier belangrijk zijn geweest tijdens mijn ontdekkingstocht door de wondere wereld van de wetenschap. Zonder hun werk, steun, inzet en hulp lag dit proefschrift er nu niet. Een aantal van hen wil ik speciaal bedanken.

Marcus, bedankt voor je vertrouwen en je aanstekelijke enthousiasme. Je wist me steeds te overtuigen dat onze soms tegenvallende en soms onverwachte resultaten wel degelijk fantastisch waren. Ondanks je overvolle agenda maakte je altijd tijd vrij voor overleg en om mijn stukken snel van feedback te voorzien. Dat heb ik zeer gewaardeerd. Hopelijk kunnen we in de toekomst nog eens samenwerken. Arnoud, ik wil je bedanken voor de waardevolle en inspirerende brainstormsessies. Die waren een belangrijke aanvulling op de dagelijkse begeleiding. Ik heb veel gehad aan je goede ideeën en correcties van manuscripten. Annie, dank voor je onmiskenbare bijdrage aan het gehele onderzoek. Door je optimisme en vrolijkheid wist je deelnemers voor de depressiedotcom-studie te enthousiasmeren en motiveren. Daar heb ik veel van geleerd. Bedankt voor de fijne tijd en samenwerking. Sylvia, hoewel je pas later bij dit onderzoeksproject kwam werken, hoorde je er meteen helemaal bij. Ik kan me geen aangenamere collega-depressiedotcom-onderzoeker voorstellen. We hebben wetenschappelijk lief en leed gedeeld en daarom ben ik blij dat je mijn paranimf wilt zijn. Greet en Rosanne, bedankt voor jullie bijdrage aan de dataverzameling. Dank ook voor de nodige gezelligheid. Daar zijn jullie beiden een ster in.

De depressiedotcom-studie was nooit een succes geworden zonder de bijdrage van alle deelnemers. Het belangeloos meewerken aan een onderzoek dat veel tijd en energie vergt, verdient een belangrijke plek in dit dankwoord. Ik ben alle deelnemers erkentelijk daarvoor. Ook wil ik de gemeenten Maastricht, Sittard-Geleen, Heerlen, Valkenburg, Meerssen en Eijsden bedanken voor hun bijdrage aan de rekrutering van deelnemers. Verder bedank ik alle projectgroepleden voor hun hulp en bijdrage aan manuscripten en werkoverleggen. De beoordelingscommissie wil ik bedanken voor het zorgvuldig beoordelen van mijn proefschrift en het goedkeuren ervan natuurlijk.

Karin, bedankt dat je ook een van m'n paranimfen wilt zijn. Jij was erbij toen mijn enthousiasme voor het doen van onderzoek ontstond. Ik kijk nog altijd met veel plezier terug op ons wetenschappelijk avontuur in Brighton. Susanne, bedankt voor het ontwerpen van de omslag van mijn proefschrift en voor het verzorgen van de lay-out. Het is een prachtig geheel geworden.

Pa en ma, met twee schrijvende ouders kon ik natuurlijk niet achterblijven. Nu ligt hier ook mijn boek. Bedankt voor alle kansen en steun die jullie me altijd hebben gegeven om te komen waar ik nu ben. Daan en Anne, mijn meest favoriete broer en schoonzus, dank voor jullie betrokkenheid. Tot slot: het is gebruikelijk om degene die het belangrijkst is, het laatst te noemen. Nick, ik weet niet goed waar ik moet beginnen: bedankt voor je goede zorgen, voor het samen genieten van lekker eten en goede wijn, of voor je liefdevolle hulp bij alle promotie beslommeringen? Daarom laat ik het bij: bedankt dat je er bent!

Esther 



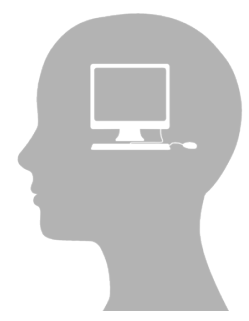

\section{Curriculum Vitae}




\section{About the author}

Esther de Graaf was born on June 29, 1980 in Amsterdam, the Netherlands. After completing secondary school in 1998 (Christelijk Gymnasium Utrecht), she lived for several months in Barcelona, Spain, taking Spanish classes. In 1999, she began her studies in Health Sciences, specializing in Mental Health Science, at Maastricht University, the Netherlands. During this time she took several additional courses in Work \& Health and in Psychology. During the years of her studies, she was also employed as a student tutor within the educational system of Maastricht University. In 2003 she lived in England for six months working as a research intern with the School of Cognitive and Computing Sciences, Sussex University, Brighton. After returning to the Netherlands in 2004, she did an additional six-month clinical internship at the department of Elderly Care of the Community Mental Health Centre (RIAGG) in Maastricht. In 2004 she received her Master's degree. From 2004 to 2009, she worked as a Ph.D. student at the department of Clinical Psychological Science at Maastricht University. Her research results are presented in this thesis. Since February 2009, she works as a post-doctoral researcher and university teacher at the department of Medical Psychology and Psychotherapy of the Erasmus MC (University Medical Center) in Rotterdam, the Netherlands.

\section{List of publications}

de Graaf, LE, Gerhards, SAH, Metsemakers, JFM, Riper, H \& Huibers, MJH (2007). De computer als hulpverlener bij depressie en angst. Bijblijven 23, 41-47.

de Graaf, LE, Gerhards, SAH, Evers, SMAA, Arntz, A, Riper, H, Severens, JL, Widdershoven, G, Metsemakers, JFM \& Huibers, MJH (2008). Clinical and costeffectiveness of computerized cognitive behavioural therapy for depression in primary care: design of a randomized trial. BMC Public Health 8, 224.

de Graaf, LE, Gerhards, SAH, Arntz, A, Riper, H, Metsemakers, JFM, Evers, SMAA, Severens, JL, Widdershoven, G \& Huibers, MJH (2009). Clinical effectiveness of online computerized cognitive behavioural therapy without support for depression in primary care: a randomized trial. British Journal of Psychiatry 195, 73-80.

de Graaf, LE, Huibers, MJH, Riper, H, Gerhards, SAH, \& Arntz, A (2009). Use and acceptability of unsupported online computerized cognitive behavioural therapy for depression and associations with clinical outcome. Journal of Affective Disorders 116, 227-231.

de Graaf, LE, Roelofs, J \& Huibers, MJH (2009). Measuring dysfunctional attitudes in the general population: the Dysfunctional Attitude Scale (form A) revised. Cognitive Therapy and Research 33, 345-355.

de Graaf, LE \& Huibers, MJH (in press). Introductie van de Diagnostic Inventory for Depression in Nederland. Tijdschrift voor Psychiatrie. 


\section{Submitted manuscripts}

de Graaf, LE, Huibers, MJH, Cuijpers, P \& Arntz, A. Minor and major depression in the general population: does dysfunctional thinking play a role?

Huibers, MJH, de Graaf, LE, Peeters, F \& Arntz, A. Does the weather make us sad? Meteorological determinants of mood and depression in the general population.

de Graaf, LE, Gerhards, SAH, Arntz, A, Riper, H, Metsemakers, JFM, Evers, SMAA, Severens, JL, Widdershoven, G \& Huibers, MJH. One-year follow-up results of unsupported online computerised cognitive behavioural therapy for depression in primary care: a randomized trial.

de Graaf, LE, Hollon, SD \& Huibers, MJH. Predictors of outcome of computerised cognitive behavioural therapy for depression in primary care: a randomized trial.

Gerhards, SAH, de Graaf, LE, Jacobs, LE, Severens, JL, Huibers, MJH, Arntz, A, Riper, $H$, Widdershoven, $G$, Metsemakers, JFM \& Evers, SMAA. Economic evaluation of online computerized cognitive behavioural therapy without support for depression in primary care: a randomized trial.

de Graaf, LE, Gerhards,SAH, Arntz, A, Riper, H, Metsemakers, JFM, Evers, SMAA, Severens, JL, Widdershoven, G \& Huibers, MJH. Klinische effectiviteit van online gecomputeriseerde cognitieve gedragstherapie zonder begeleiding voor eerstelijnsbehandeling van depressie: een gerandomiseerd onderzoek. 